\title{
SWEET \& SOUR AND OTHER FLAVOURS OF CCC FORCING NOTIONS
}

\author{
ANDRZEJ ROSŁANOWSKI AND SAHARON SHELAH
}

\begin{abstract}
We continue developing the general theory of forcing notions built with the use of norms on possibilities, this time concentrating on ccc forcing notions and classifying them.
\end{abstract}

\section{Contents}

0. Introduction

0.1. The content of the paper

0.2. Notation

1. Building Souslin ccc forcing notions

1.1. Glue and cut - the method of [RoSh:628]

1.2. Tree-like conditions 8

1.3. The complexity of our forcing notions 9

\begin{tabular}{|r|r|r|r|}
\hline 1.4. & Unbounded and dominating reals & 12
\end{tabular}

$\begin{array}{lll}1.5 . & \text { Examples } & 18\end{array}$

$\begin{array}{lll}2 . & \text { More constructions } & 27\end{array}$

2.1. Mixtures with random 28

2.2. Exotic norm conditions 33

$\begin{array}{lll}2.3 . & \text { Universal forcing notions } & 37\end{array}$

\begin{tabular}{ll|l}
2.4. & Examples & 39
\end{tabular}

3. Interlude: ideals 44

3.1. Generic ideals 44

3.2. Universality ideal 46

\begin{tabular}{ll|l}
3.3. & Baire Property & 47
\end{tabular}

$\begin{array}{ll}4 . \quad \text { Sweet and Sour } & 48\end{array}$

$\begin{array}{lll}4.1 . & \text { On sweetness } & 48\end{array}$

$\begin{array}{lll}\text { 4.2. } & \text { The sour part of the spectrum } & 57\end{array}$

4.3. Conclusions 65

$\begin{array}{ll}\text { 5. Epilogue } & 66\end{array}$

$\begin{array}{ll}\text { References } & 68\end{array}$

Date: November 2001.

1991 Mathematics Subject Classification. Primary 03E35; Secondary 03E40, 03E05.

The first author thanks the Hebrew University of Jerusalem for their hospitality during his visits to Jerusalem in Summer'00 and Summer'01. His research was also partially supported by a grant from the University Committee on Research of UNO.

The research of the second author was partially supported by the Israel Science Foundation. Publication 672. 


\section{INTRODUCTION}

The present paper has three themes. First, we continue the investigations started in Judah, Rosłanowski and Shelah [8] and Rosłanowski and Shelah [15], [16], and we investigate the method of norms on possibilities in the context of ccc forcing notions, getting a number of constructions of nicely definable ccc forcings. Most of them fall into the class of nep-forcing notions of Shelah [23], 24] (giving yet more examples to which the general theory developed there can be applied).

The second theme of the paper is a part of the general program "how special are random and Cohen forcing notions (or: the respective ideals)". Kunen (see 11. Question 1.2]) suspected that the null ideal and the meager ideal on $2^{\omega}$ can be somehow characterized by their combinatorial properties, but in [16] we constructed $\sigma$-ideals (or rather forcing notions) that have nice properties, however are different from the two. (But see also Kechris and Solecki [10] and Solecki [29] for results in the opposite direction.) Shelah [21] shows that the two forcing notions may occupy special positions in the realm of nicely definable forcing notions. In this realm we may classify forcing notions using the methods of 23], 24 and, for example, declare that very Souslin (or generally $\omega$-nw-nep) ccc forcing notions (see 1.3.1) are really nice. Both the Cohen forcing notion and the random forcing notion and their FS iterations (and nice subforcings) are all ccc $\omega-\mathrm{nw}-\mathrm{nep}$, and [22, Problem $4.24]$ asked if we have more examples. It occurs that our method relatively easily results in very Souslin ccc forcing notions (see 1.3.4 (3), 1.5.8 (2), 1.5.11, 1.5.15(3)).

The third theme is sweet $\&$ sour and it is related to one of the most striking differences between the random and the Cohen forcing notions which appears when we consider the respective regularity properties of projective set. In [20], Shelah proved that the Lebesgue measurability of $\Sigma_{3}^{1}$ sets implies $\omega_{1}$ is inaccessible in $\mathbf{L}$, while one can construct (in ZFC) a forcing notion $\mathbb{P}$ such that $\mathbf{V}^{\mathbb{P}} \models$ "projective subsets of $\mathbb{R}$ have the Baire property ". The latter construction involved a strong version of ccc, so called "sweetness" (see 4.1.2). The heart of the former result is that the composition of two Amoeba for measure forcing notions is sour (see 4.2.2) over random. Also from a sequence of $\omega_{1}$ reals we can define a non-measurable set, but not one without the Baire Property.

It seems that sweet-sour properties of forcing notions could be used to classify them as either close to Cohen or as more random-like. Again, our methods result in examples for both cases.

Let us postpone the discussion of the general context of this paper till Epilogue, when we can easier refer to the definitions and notions discussed in the paper. (But the curious reader may start reading this paper from that section.)

We try to make this work self contained, citing the most important definitions and results from [15], 16] whenever needed. However, at least superficial familiarity with those papers could be of some help in reading this paper.

0.1. The content of the paper. Like in [15], the basic intention of this paper is to present "the general theory" rather than particular examples. Therefore, we extract those properties of an example we want to construct which are responsible for the fact that it works and we separate "the general theory" from its applications. But to make the paper more readable, in most cases, we sacrifice generality for clarity.

In the first section we uniformize and generalize the constructions of [8] and [16]. We investigate the complexity of the resulting forcing notions as well as properties like "adding unbounded reals", "preserving unbounded families", etc. 
The next section introduces more ways in which creatures (or tree-creatures) can be used to build ccc forcing notions. We discuss mixtures with randoms, some generalizations of the Amoeba for Category forcing notion, as well as as "artificial" modifications of previously introduced forcings.

The third part formalizes definitions of $\sigma$-ideals corresponding to our forcing notions.

The following section discusses sweet-sour properties of our forcing notions. We recall the notions of sweetness and introduce yet another sweet property, and we show that very often our constructions are (somewhat) sweet. However, there are exceptions to this rule. So we define some strong negations of sweetness (sourness) and we show how our schema may end up with very sour results.

Finally, the last section is (in some sense) a continuation of the introduction. We discuss the results of the paper and formulate some problems.

0.2. Notation. Most of our notation is standard and compatible with that of classical textbooks on Set Theory (like Bartoszyński and Judah [1]). However in forcing we keep the convention that a stronger condition is the larger one.

Basic Notation: In this paper $\mathbf{H}$ will stand for a function with domain $\omega$ and such that $(\forall m \in \omega)(|\mathbf{H}(m)| \geq 2)$. We usually assume that $0 \in \mathbf{H}(m)$ (for all $m \in \omega)$; if it is not the case then we fix an element of $\mathbf{H}(m)$ and we use it whenever appropriate notions refer to 0 . Moreover we demand $\mathbf{H} \in \mathcal{H}\left(\omega_{1}\right)$ (i.e., $\mathbf{H}$ is hereditarily countable.

\section{MORE NotATiOn:}

1. $\mathbb{R}^{\geq 0}$ stands for the set of non-negative reals. The integer part of a real $r \in \mathbb{R}^{\geq 0}$ is denoted by $\lfloor r\rfloor$.

2. For two sequences $\eta, \nu$ we write $\nu \triangleleft \eta$ whenever $\nu$ is a proper initial segment of $\eta$, and $\nu \unlhd \eta$ when either $\nu \triangleleft \eta$ or $\nu=\eta$. The length of a sequence $\eta$ is denoted by $\ln (\eta)$.

3. A tree is a family $T$ of finite sequences such that for some $\operatorname{root}(T) \in T$ we have

$$
(\forall \nu \in T)(\operatorname{root}(T) \unlhd \nu) \quad \text { and } \quad \operatorname{root}(T) \unlhd \nu \unlhd \eta \in T \Rightarrow \nu \in T .
$$

For a tree $T$, the family of all $\omega$-branches through $T$ is denoted by $[T]$, and we let

$$
\max (T) \stackrel{\text { def }}{=}\{\nu \in T: \text { there is no } \rho \in T \text { such that } \nu \triangleleft \rho\} .
$$

If $\eta$ is a node in the tree $T$ then

$$
\begin{array}{ll}
\operatorname{succ}_{T}(\eta) & =\{\nu \in T: \eta \triangleleft \nu \& \operatorname{lh}(\nu)=\operatorname{lh}(\eta)+1\} \text { and } \\
T^{[\eta]} & =\{\nu \in T: \eta \unlhd \nu\} .
\end{array}
$$

4. The quantifiers $\left(\forall^{\infty} n\right)$ and $\left(\exists^{\infty} n\right)$ are abbreviations for

$$
(\exists m \in \omega)(\forall n>m) \quad \text { and } \quad(\forall m \in \omega)(\exists n>m),
$$

respectively.

5. For a set $X, \quad[X] \leq \omega,[X]<\omega$ and $\mathcal{P}(X)$ will stand for families of countable, finite and all, respectively, subsets of the set $X$. The family of $k$-element subsets of $X$ will be denoted by $[X]^{k}$. The set of all finite sequences with values in $X$ is called $X^{<\omega}$ (so domains of elements of $X^{<\omega}$ are integers). The collection of all finite partial functions from $\omega$ to $X$ is $X \omega$. 
6. For a relation $R$ (a set of ordered pairs), $\operatorname{dom}(R)$ stands for the domain of $R$ and $\operatorname{rng}(R)$ denotes the range of $R$.

7. The Cantor space $2^{\omega}$ and the Baire space $\omega^{\omega}$ are the spaces of all functions from $\omega$ to $2, \omega$, respectively, equipped with natural (Polish) topology.

8. For $f, g \in \omega^{\omega}$ we write $f<^{*} g\left(f \leq^{*} g\right.$, respectively) whenever $\left(\forall^{\infty} n \in\right.$ $\omega)(f(n)<g(n))\left(\left(\forall^{\infty} n \in \omega\right)(f(n) \leq g(n))\right.$, repectively $)$.

9. For a forcing notion $\mathbb{P}, \Gamma_{\mathbb{P}}$ stands for the canonical $\mathbb{P}$-name for the generic filter in $\mathbb{P}$. With this one exception, all $\mathbb{P}$-names for objects in the extension via $\mathbb{P}$ will be denoted with a dot above (e.g. $\dot{\tau}, \dot{X}$ ). The weakest element of $\mathbb{P}$ will be denoted by $\emptyset_{\mathbb{P}}$ (and we will always assume that there is one, and that there is no other condition equivalent to it).

\section{Building Souslin CCC FORCing NOtions}

In this section we will review methods for building ccc forcing notions announced or present in some form in [8], [15], and [16].

1.1. Glue and cut - the method of [RoSh:628]. Here we re-present the method of building ccc forcing notions with use of (semi-) creating triples from 16. We will slightly modify the definitions loosing some generality. However, we will gain more direct connection to the method of [15] and (hopefully) a better clarity of arguments. Note that the main difference is that here we do not worry about "the permutation invariance" of our forcing notions, so the creatures get back their $m_{\mathrm{dn}}^{t}, m_{\mathrm{up}}^{t}$ (and they are like those of 15]).

Definition 1.1.1. Let $\mathbf{H}: \omega \longrightarrow \mathcal{H}\left(\omega_{1}\right)$.

1. (See [15, Def. 1.1.1, 1.2.1]) A creature for $\mathbf{H}$ is a triple

$$
t=(\text { nor }, \operatorname{val}, \operatorname{dis})=(\operatorname{nor}[t], \operatorname{val}[t], \operatorname{dis}[t])
$$

such that nor $\in \mathbb{R}^{\geq 0}$, dis $\in \mathcal{H}\left(\omega_{1}\right)$, and for some integers $m_{\mathrm{dn}}^{t}<m_{\mathrm{up}}^{t}<\omega$

$$
\emptyset \neq \mathbf{v a l} \subseteq\left\{\langle u, v\rangle \in \prod_{i<m_{\mathrm{dn}}^{t}} \mathbf{H}(i) \times \prod_{i<m_{\mathrm{up}}^{t}} \mathbf{H}(i): u \triangleleft v\right\} .
$$

The set of all creatures for $\mathbf{H}$ will be denoted by $\mathrm{CR}[\mathbf{H}]$, and for $m_{0}<m_{1}<\omega$ we let $\mathrm{CR}_{m_{0}, m_{1}}[\mathbf{H}]=\left\{t \in \mathrm{CR}[\mathbf{H}]: m_{\mathrm{dn}}^{t}=m_{0} \& m_{\mathrm{up}}^{t}=m_{1}\right\}$.

2. (See 15, Def. 1.1.4, 1.2.2, 1.2.5]) Let $K \subseteq \mathrm{CR}[\mathbf{H}]$. We say that a function $\Sigma:[K]^{<\omega} \longrightarrow \mathcal{P}(K)$ is a composition operation on $K$ whenever the following conditions are satisfied.

(a) If $\mathcal{S} \in[K]^{<\omega}$ and $\Sigma(\mathcal{S}) \neq \emptyset$, then for some enumeration $\mathcal{S}=\left\{t_{0}, \ldots, t_{k}\right\}$ we have $m_{\mathrm{up}}^{t_{i}}=m_{\mathrm{dn}}^{t_{i+1}}$ for all $i<k$ [from now on, whenever we write $\Sigma\left(t_{0}, \ldots, t_{k}\right)$, we mean the enumeration in which $\left.m_{\mathrm{up}}^{t_{i}}=m_{\mathrm{dn}}^{t_{i+1}}\right]$, and

(b) if $s \in \Sigma\left(t_{0}, \ldots, t_{k}\right)$, then $m_{\mathrm{dn}}^{s}=m_{\mathrm{dn}}^{t_{0}}$ and $m_{\mathrm{up}}^{s}=m_{\mathrm{up}}^{t_{k}}$, and

(c) $t \in \Sigma(t)$ for each $t \in K, \Sigma(\emptyset)=\emptyset$, and

(d) [transitivity] if $s_{i} \in \Sigma\left(t_{0}^{i}, \ldots, t_{k_{i}}^{i}\right)$ (for $\left.i \leq n\right)$, then

$$
\Sigma\left(s_{0}, \ldots, s_{n}\right) \subseteq \Sigma\left(t_{j}^{i}: i \leq n \& j \leq k_{i}\right),
$$

(e) [niceness 8 smoothness] if $s \in \Sigma\left(t_{0}, \ldots, t_{k}\right), m_{\mathrm{up}}^{t_{i}}=m_{\mathrm{dn}}^{t_{i+1}}$ (for $\left.i<k\right)$, then $\operatorname{dom}\left(\operatorname{val}\left[t_{0}\right]\right)=\operatorname{dom}(\operatorname{val}[s])$ and

$$
(\forall\langle u, v\rangle \in \operatorname{val}[s])(\forall i \leq k)\left(\left\langlev\left\lceil m_{\mathrm{dn}}^{t_{i}}, v\left\lceil m_{\mathrm{up}}^{t_{i}}\right\rangle \in \operatorname{val}\left[t_{i}\right]\right) .\right.\right.
$$


3. (See [16, Def. 1.1]) A function $\Sigma^{\perp}: K \longrightarrow[K]^{<\omega} \backslash\{\emptyset\}$ is called a decomposition operation on $K$ if for each $t \in K$ :

$(\mathrm{a})^{\perp}$ if $\mathcal{S} \in \Sigma^{\perp}(t)$, then for some enumeration $\mathcal{S}=\left\{s_{0}, \ldots, s_{k}\right\}$ we have $m_{\text {up }}^{s_{i}}=$ $m_{\mathrm{dn}}^{s_{i+1}}$ (for $i<k$ ) [from now on, if we write $\left\{s_{0}, \ldots, s_{k}\right\} \in \Sigma^{\perp}(t)$, we mean the enumeration in which $m_{\mathrm{up}}^{s_{i}}=m_{\mathrm{dn}}^{s_{i+1}}$, and

(b) ${ }^{\perp}$ if $\left\{s_{0}, \ldots, s_{k}\right\} \in \Sigma^{\perp}(t)$ then $m_{\mathrm{dn}}^{s_{0}}=m_{\mathrm{dn}}^{t}, m_{\mathrm{up}}^{s_{k}}=m_{\mathrm{up}}^{t}$

(c) $)^{\perp}\{t\} \in \Sigma^{\perp}(t)$,

(d) ${ }^{\perp}$ [transitivity $]$ if $\mathcal{S}=\left\{s_{0}, \ldots, s_{k}\right\} \in \Sigma^{\perp}(t)$ and $\mathcal{S}_{i} \in \Sigma^{\perp}\left(s_{i}\right)$ (for $i \leq k$ ), then $\mathcal{S}_{0} \cup \ldots \cup \mathcal{S}_{k} \in \Sigma^{\perp}(t)$,

$(\mathrm{e})^{\perp}$ if $\left\{s_{0}, \ldots, s_{k}\right\} \in \Sigma^{\perp}(t), m_{\mathrm{up}}^{s_{i}}=m_{\mathrm{dn}}^{s_{i+1}}$ (for $\left.i<k\right)$, then

$\operatorname{dom}(\operatorname{val}[t])=\operatorname{dom}\left(\operatorname{val}\left[s_{0}\right]\right)$ and $(\forall i<k)\left(\operatorname{rng}\left(\operatorname{val}\left[s_{i}\right]\right) \subseteq \operatorname{dom}\left(\operatorname{val}\left[s_{i+1}\right]\right)\right)$,

and

$\left\{\langle u, v\rangle: u \in \operatorname{dom}\left(\operatorname{val}\left[s_{0}\right]\right) \& u \triangleleft v \&(\forall i \leq k)\left(\langle v| m_{\mathrm{dn}}^{s_{i}}, v\left\lceil m_{\mathrm{up}}^{s_{i}}\right\rangle \in \operatorname{val}\left[s_{i}\right]\right)\right\} \subseteq \operatorname{val}[t]$.

4. If $K \subseteq \mathrm{CR}[\mathbf{H}]$ and $\Sigma$ is a composition operation on $K$, then $(K, \Sigma)$ is called $a$ creating pair for $\mathbf{H}$. If, additionally, $\Sigma^{\perp}$ is a decomposition operation on $K$, then $\left(K, \Sigma, \Sigma^{\perp}\right)$ is called $a \otimes$-creating triple for $\mathbf{H}$.

5. If $t_{0}, \ldots, t_{n} \in K$ are such that $m_{\mathrm{up}}^{t_{i}}=m_{\mathrm{dn}}^{t_{i+1}}$ (for $\left.i<n\right)$ and $w \in \operatorname{dom}\left(\operatorname{val}\left[t_{0}\right]\right)$, then we let

$\operatorname{pos}\left(w, t_{0}, \ldots, t_{n}\right) \stackrel{\text { def }}{=}\left\{v \in \prod_{j<m_{\mathrm{up}}^{t_{n}}} \mathbf{H}(j): w \triangleleft v \&(\forall i \leq n)\left(\left\langle v\left\lceil m_{\mathrm{dn}}^{t_{i}}, v\left\lceil m_{\mathrm{up}}^{t_{i}}\right\rangle \in \mathbf{v a l}\left[t_{i}\right]\right)\right\}\right.\right.$.

Definition 1.1.2. Let $\left(K, \Sigma, \Sigma^{\perp}\right)$ be a $\otimes$-creating triple for $\mathbf{H}$. We say that

1. $\Sigma^{\perp}$ is trivial if $\Sigma^{\perp}(t)=\{\{t\}\}$ for each $t \in K$;

2. $(K, \Sigma)$ is simple if $\Sigma(\mathcal{S})$ is non-empty for singletons only; if additionally $\Sigma^{\perp}$ is trivial, then we say that $\left(K, \Sigma, \Sigma^{\perp}\right)$ is simple;

3. $K$ (or $(K, \Sigma)$ or $\left.\left(K, \Sigma, \Sigma^{\perp}\right)\right)$ is local if $m_{\mathrm{up}}^{t}=m_{\mathrm{dn}}^{t}+1$ for each creature $t \in K$ (so then necessarily $\left(K, \Sigma, \Sigma^{\perp}\right)$ is simple);

4. $K$ is forgetful if for every creature $t \in K$ we have

$$
\left[\langle u, v\rangle \in \operatorname{val}[t] \& w \in \prod_{i<m_{\mathrm{dn}}^{t}} \mathbf{H}(i)\right] \Rightarrow\left\langle w, w^{\frown} v \uparrow\left[m_{\mathrm{dn}}^{t}, m_{\mathrm{up}}^{t}\right)\right\rangle \in \mathbf{v a l}[t] ;
$$

5. $K$ is full if $\operatorname{dom}(\operatorname{val}[t])=\prod_{i<m_{\mathrm{dn}}^{t}} \mathbf{H}(i)$ for each $t \in K$.

Definition 1.1.3. [See [15, Def. 1.1.7, 1.2.6], [16, Def. 1.3]] Let $\left(K, \Sigma, \Sigma^{\perp}\right)$ be a $\otimes$-creating triple for $\mathbf{H}$ and let $\mathcal{C}($ nor $)$ be a property of $\omega$-sequences of creatures from $K$ (so $\mathcal{C}\left(\right.$ nor) can be thought of as a subset of $K^{\omega}$ ). We define a forcing notion $\mathbb{Q}_{\mathcal{C}(\text { nor })}^{*}\left(K, \Sigma, \Sigma^{\perp}\right)$ as follows.

A condition in $\mathbb{Q}_{\mathcal{C}(\text { nor })}^{*}\left(K, \Sigma, \Sigma^{\perp}\right)$ is a sequence $p=\left(w^{p}, t_{0}^{p}, t_{1}^{p}, t_{2}^{p}, \ldots\right)$ such that

(a) $t_{i}^{p} \in K$ and $m_{\mathrm{up}}^{t_{i}^{p}}=m_{\mathrm{dn}}^{t_{i+1}^{p}}($ for $i<\omega)$,

(b) $w \in \operatorname{dom}\left(\operatorname{val}\left[t_{0}^{p}\right]\right)$ and $\left\langle t_{0}^{p}, t_{1}^{p}, t_{2}^{p}, \ldots\right\rangle \in \mathcal{C}($ nor $)$,

(c) $\operatorname{pos}\left(w^{p}, t_{0}^{p}, \ldots, t_{i}^{p}\right) \subseteq \operatorname{dom}\left(\operatorname{val}\left[t_{i+1}^{p}\right]\right)$ for each $i<\omega$. 
$\mathbb{Q}_{\emptyset}^{*}\left(K, \Sigma, \Sigma^{\perp}\right)$ is defined similarly, but we skip the demand " $\left\langle t_{0}^{p}, t_{1}^{p}, \ldots\right\rangle \in \mathcal{C}($ nor $)$ " in clause (b) above (or we just let $\mathcal{C}($ nor $)=K^{\omega}$; it is perhaps unfortunate to use $\emptyset$ in this context, but that notation was established in [15).

The relation $\leq$ on $\mathbb{Q}_{\mathcal{C}(\text { nor })}^{*}\left(K, \Sigma, \Sigma^{\perp}\right)$ is given by: $p \leq q$ if and only if $\left(w^{q}, t_{0}^{q}, t_{1}^{q}, t_{2}^{q}, \ldots\right)$ can be obtained from $\left(w^{p}, t_{0}^{p}, t_{1}^{p}, t_{2}^{p}, \ldots\right)$ by applying finitely many times the following operations (describing the operations, we say what are the results of applying the operation to a condition $\left.\left(w, t_{0}, t_{1}, t_{2}, \ldots\right) \in \mathbb{Q}_{\emptyset}^{*}\left(K, \Sigma, \Sigma^{\perp}\right)\right)$.

Deciding the value for $\left(w, t_{0}, t_{1}, t_{2}, \ldots\right)$ :

a result of this operation is a condition $\left(w^{*}, t_{n}, t_{n+1}, t_{n+2}, \ldots\right) \in \mathbb{Q}_{\emptyset}^{*}\left(K, \Sigma, \Sigma^{\perp}\right)$ such that $w^{*} \in \operatorname{pos}\left(w, t_{0}, \ldots, t_{n-1}\right)$ for some $n<\omega$.

Applying $\Sigma$ to $\left(w, t_{0}, t_{1}, t_{2}, \ldots\right)$ :

a result of this operation is a condition $\left(w, t_{0}^{*}, t_{1}^{*}, t_{2}^{*}, \ldots\right) \in \mathbb{Q}_{\emptyset}^{*}\left(K, \Sigma, \Sigma^{\perp}\right)$ such that for some increasing sequence $0=n_{0}<n_{1}<n_{2}<\ldots<\omega$, for each $i<\omega$, we have $t_{i}^{*} \in \Sigma\left(t_{n_{i}}, \ldots, t_{n_{i+1}-1}\right)$.

Applying $\Sigma^{\perp}$ to $\left(w, t_{0}, t_{1}, t_{2}, \ldots\right)$ :

a result of this operation is a condition $\left(w, t_{0}^{*}, t_{1}^{*}, t_{2}^{*}, \ldots\right) \in \mathbb{Q}_{\emptyset}^{*}\left(K, \Sigma, \Sigma^{\perp}\right)$ such that for some increasing sequence $0=n_{0}<n_{1}<n_{2}<\ldots<\omega$, for each $i<\omega$, we have $\left\{t_{n_{i}}^{*}, \ldots, t_{n_{i+1}-1}^{*}\right\} \in \Sigma^{\perp}\left(t_{i}\right)$.

Remark 1.1.4. In the definition of the relation $\leq$ on $\mathbb{Q}_{\mathcal{C}(\text { nor })}^{*}\left(K, \Sigma, \Sigma^{\perp}\right)$ we do not require that the intermediate steps satisfy the norm condition $\mathcal{C}($ nor $)$. So it may happen that the sequence of witnesses for $p \leq q$ (i.e., the results of the respective operations) is not in $\mathbb{Q}_{\mathcal{C}(\text { nor })}^{*}\left(K, \Sigma, \Sigma^{\perp}\right)$.

If $\Sigma^{\perp}$ is trivial we may omit it; note that then we are exactly in the setting of 15, $\S 1.2]$.

Definition 1.1.5. We will consider the following norm conditions $\mathcal{C}($ nor $)$ :

- A sequence $\left\langle t_{i}: i<\omega\right\rangle$ satisfies $\mathcal{C}^{\infty}$ (nor) if $\lim _{i \rightarrow \infty} \operatorname{nor}\left[t_{i}\right]=\infty$

[the respective forcing notion is called $\mathbb{Q}_{\infty}^{*}\left(K, \Sigma, \Sigma^{\perp}\right)$ ].

- Let $\mathcal{F} \subseteq \omega^{\omega}$; a sequence $\left\langle t_{i}: i<\omega\right\rangle$ satisfies $\mathcal{C}^{\mathcal{F}}$ (nor) if

$$
(\exists f \in \mathcal{F})\left(\forall^{\infty} i \in \omega\right)\left(\operatorname{nor}\left[t_{i}\right] \geq f\left(m_{\mathrm{dn}}^{t_{i}}\right)\right)
$$

[the respective forcing notion is denoted $\mathbb{Q}_{\mathcal{F}}^{*}\left(K, \Sigma, \Sigma^{\perp}\right)$ ].

- Let $f: \omega \times \omega \longrightarrow \omega$; a sequence $\left\langle t_{i}: i<\omega\right\rangle$ satisfies $\mathcal{C}^{f}$ (nor) if

$$
(\forall n \in \omega)\left(\forall^{\infty} i \in \omega\right)\left(\operatorname{nor}\left[t_{i}\right] \geq f\left(n, m_{\mathrm{dn}}^{t_{i}}\right)\right)
$$

[the respective forcing notion is denoted $\mathbb{Q}_{f}^{*}\left(K, \Sigma, \Sigma^{\perp}\right)$ ].

We will consider the norm conditions $\mathcal{C}^{\mathcal{F}}$ (nor), $\mathcal{C}^{f}$ (nor) only for $h$-closed families $\mathcal{F}$ and fast functions $f$, see 1.1 .6 below. Later we will introduce more methods for building ccc forcing notions, including more norm conditions.

Definition 1.1.6. 1. A function $f: \omega \times \omega \longrightarrow \omega$ is fast if

$$
(\forall k, \ell \in \omega)(f(k, \ell) \leq f(k, \ell+1) \& 2 \cdot f(k, \ell)<f(k+1, \ell)) .
$$

2. A function $h: \omega \times \omega \longrightarrow \omega$ is regressive if

$$
(\forall m \in \omega)((\forall k>1)(1 \leq h(m, k)<k) \&(\forall k<\ell<\omega)(h(m, k) \leq h(m, \ell))) .
$$


3. Let $h: \omega \times \omega \longrightarrow \omega$. We say that a family $\mathcal{F} \subseteq \omega^{\omega}$ is $h$-closed if for every $f \in \mathcal{F}$ there is $f^{*} \in \mathcal{F}$ such that $\left(\forall^{\infty} n \in \omega\right)\left(f^{*}(n) \leq h(n, f(n))\right)$.

4. A family $\mathcal{F} \subseteq \omega^{\omega}$ is $\geq^{*}$-directed if

$$
\left(\forall f_{0}, f_{1} \in \mathcal{F}\right)\left(\exists f^{*} \in \mathcal{F}\right)\left(\forall^{\infty} n \in \omega\right)\left(f^{*}(n) \leq \min \left\{f_{0}(n), f_{1}(n)\right\}\right) .
$$

Similarly we define $\leq^{*}$-directed families (just reversing the inequality).

Remark 1.1.7. Let $f(n, m)=2^{2 n}$ (for $\left.n, m \in \omega\right)$. Then the function $f$ is fast and the norm conditions $\mathcal{C}^{f}$ (nor) and $\mathcal{C}^{\infty}($ nor $)$ agree (and thus $\mathbb{Q}_{f}^{*}(K, \Sigma)=\mathbb{Q}_{\infty}^{*}(K, \Sigma)$ for a local creating pair $(K, \Sigma))$. In practical applications, when we consider the norm condition $\mathcal{C}^{f}$ (nor), the function $f$ is such that $f(n, m)<f(n, m+1)$ (for all $n, m \in \omega)$ and thus the norm condition $\mathcal{C}^{f}$ (nor) is stronger than $\mathcal{C}^{\infty}$ (nor).

Proposition 1.1.8. If $\left(K, \Sigma, \Sigma^{\perp}\right)$ is a $\otimes$-creating triple for $\mathbf{H}, \mathcal{C}($ nor $) \subseteq K^{\omega}$, then $\mathbb{Q}_{\mathcal{C}(\text { nor })}^{*}\left(K, \Sigma, \Sigma^{\perp}\right)$ is a forcing notion (i.e., the relation $\leq$ of $\mathbb{Q}_{\mathcal{C}(\text { nor })}^{*}\left(K, \Sigma, \Sigma^{\perp}\right)$ is transitive).

Definition 1.1.9. [See [15, Def. 1.2.4]] Let $\left(K, \Sigma, \Sigma^{\perp}\right)$ be a $\otimes$-creating triple for $\mathbf{H}$. We define finite candidates $(\mathrm{FC})$ and pure finite candidates $(\mathrm{PFC})$ with respect to $\left(K, \Sigma, \Sigma^{\perp}\right)$ :

$$
\begin{aligned}
\mathrm{FC}\left(K, \Sigma, \Sigma^{\perp}\right)= & \left\{\left(w, t_{0}, \ldots, t_{n}\right): w \in \operatorname{dom}\left(\operatorname{val}\left[t_{0}\right]\right) \text { and for each } i \leq n:\right. \\
& \left.t_{i} \in K, m_{\mathrm{up}}^{t_{i}}=m_{\mathrm{dn}}^{t_{i+1}} \text { and } \operatorname{pos}\left(w, t_{0}, \ldots, t_{i}\right) \subseteq \operatorname{dom}\left(\operatorname{val}\left[t_{i+1}\right]\right)\right\},
\end{aligned}
$$

$\operatorname{PFC}\left(K, \Sigma, \Sigma^{\perp}\right)=\left\{\left(t_{0}, \ldots, t_{n}\right):\left(\exists w \in \operatorname{dom}\left(\operatorname{val}\left[t_{0}\right]\right)\right)\left(\left(w, t_{0}, \ldots, t_{n}\right) \in \operatorname{FC}\left(K, \Sigma, \Sigma^{\perp}\right)\right)\right\}$.

We have a natural partial order $\leq$ on $\mathrm{FC}\left(K, \Sigma, \Sigma^{\perp}\right)$ (like in 1.1.3). [Note that $\Sigma, \Sigma^{\perp}$ have no influence on $\mathrm{FC}\left(K, \Sigma, \Sigma^{\perp}\right)$, that is they are not present in the definition of finite candidates, and we could have written $\mathrm{FC}(K, \Sigma)$ or $\mathrm{FC}(K)$. However, they come to game when the partial order $\leq$ on $\mathrm{FC}\left(K, \Sigma, \Sigma^{\perp}\right)$ is considered.]

A sequence $\left\langle t_{0}, t_{1}, t_{2}, \ldots\right\rangle$ of creatures from $K$ is a pure candidate with respect to $\left(K, \Sigma, \Sigma^{\perp}\right)$ if

$$
(\forall i<\omega)\left(m_{\mathrm{up}}^{t_{i}}=m_{\mathrm{dn}}^{t_{i+1}}\right) \quad \text { and }
$$

$$
\left(\exists w \in \operatorname{dom}\left(\operatorname{val}\left[t_{0}\right]\right)\right)(\forall i<\omega)\left(\operatorname{pos}\left(w, t_{0}, \ldots, t_{i}\right) \subseteq \operatorname{dom}\left(\operatorname{val}\left[t_{i+1}\right]\right)\right) .
$$

The set of pure candidates with respect to $(K, \Sigma)$ is denoted by $\operatorname{PC}\left(K, \Sigma, \Sigma^{\perp}\right)$. The partial order $\leq$ on $\operatorname{PC}\left(K, \Sigma, \Sigma^{\perp}\right)$ is defined naturally.

For a norm condition $\mathcal{C}($ nor $)$ the family of $\mathcal{C}($ nor $)$-normed pure candidates is $\operatorname{PC}_{\mathcal{C}(\text { nor })}\left(K, \Sigma, \Sigma^{\perp}\right)=\left\{\left\langle t_{0}, t_{1}, \ldots\right\rangle \in \operatorname{PC}\left(K, \Sigma, \Sigma^{\perp}\right):\left\langle t_{0}, t_{1}, \ldots,\right\rangle\right.$ satisfies $\mathcal{C}($ nor $\left.)\right\}$.

Definition 1.1.10. Let $\left(K, \Sigma, \Sigma^{\perp}\right)$ be a $\otimes$-creating triple for $\mathbf{H}$.

1. For a condition $p \in \mathbb{Q}_{\emptyset}^{*}\left(K, \Sigma, \Sigma^{\perp}\right)$ we let

$$
\operatorname{POS}(p) \stackrel{\text { def }}{=}\left\{u \in \bigcup_{n<\omega} \prod_{i<n} \mathbf{H}(i):(\exists \ell<\omega)\left(\exists v \in \operatorname{pos}\left(w^{p}, t_{0}^{p}, \ldots, t_{\ell}^{p}\right)\right)(u \unlhd v)\right\} .
$$

2. For a finite candidate $c=\left(w, t_{0}, \ldots, t_{k}\right) \in \mathrm{FC}\left(K, \Sigma, \Sigma^{\perp}\right)$ we define

$$
\operatorname{POS}(c) \stackrel{\text { def }}{=}\left\{u \in \bigcup_{n \leq m_{\mathrm{up}}^{t_{k}}} \prod_{i<n} \mathbf{H}(i):\left(\exists v \in \operatorname{pos}\left(w, t_{0}, \ldots, t_{k}\right)\right)(u \unlhd v)\right\} .
$$


Proposition 1.1.11. Suppose $\left(K, \Sigma, \Sigma^{\perp}\right)$ is a $\otimes$-creating triple for $\mathbf{H}$.

1. If $p, q \in \mathbb{Q}_{\emptyset}^{*}\left(K, \Sigma, \Sigma^{\perp}\right), p \leq q$ then $\operatorname{POS}(p) \subseteq \operatorname{POS}(q)$, and if $\operatorname{lh}\left(w^{q}\right)=m_{\mathrm{up}}^{t_{\ell}^{p}}$ for some $\ell<\omega$, then $w^{q} \in \operatorname{pos}\left(w^{p}, t_{0}^{p}, \ldots, t_{\ell}^{p}\right)$.

2. The same holds if one replaces conditions from $\mathbb{Q}_{\emptyset}^{*}\left(K, \Sigma, \Sigma^{\perp}\right)$ by finite candidates from $\mathrm{FC}\left(K, \Sigma, \Sigma^{\perp}\right)$.

Proof. Note that each of the three operations described in 1.1.3 shrinks POS (remember 1.1.1 $(2 \mathrm{e})$ and $\left.1.1 .1\left(3 \mathrm{e}^{\perp}\right)\right)$.

Definition 1.1.12. [See [16, Def. 2.1], [15, Def. 2.1.7]] Assume that $\left(K, \Sigma, \Sigma^{\perp}\right)$ is a $\otimes$-creating triple for $\mathbf{H}$.

1. We say that $(K, \Sigma)$ (or $\left(K, \Sigma, \Sigma^{\perp}\right)$ ) is linked if for each $t_{0}, t_{1} \in K$ such that $\operatorname{nor}\left[t_{0}\right], \operatorname{nor}\left[t_{1}\right]>1$ and $m_{\mathrm{dn}}^{t_{0}}=m_{\mathrm{dn}}^{t_{1}}, m_{\mathrm{up}}^{t_{0}}=m_{\mathrm{up}}^{t_{1}}$, there is $s \in \Sigma\left(t_{0}\right) \cap \Sigma\left(t_{1}\right)$ with

$$
\operatorname{nor}[s] \geq \min \left\{\operatorname{nor}\left[t_{0}\right], \operatorname{nor}\left[t_{1}\right]\right\}-1 \text {. }
$$

Let $h: \omega \times \omega \longrightarrow \omega$. The pair $(K, \Sigma)$ is said to be $h$-linked if for each $k>1$, and creatures $t_{0}, t_{1} \in K$ such that nor $\left[t_{0}\right], \operatorname{nor}\left[t_{1}\right] \geq k$ and $m_{\mathrm{dn}}^{t_{0}}=m_{\mathrm{dn}}^{t_{1}}$, $m_{\mathrm{up}}^{t_{0}}=m_{\mathrm{up}}^{t_{1}}$, there is $s \in \Sigma\left(t_{0}\right) \cap \Sigma\left(t_{1}\right)$ with nor $[s] \geq h\left(m_{\mathrm{dn}}^{t_{0}}, k\right)$.

2. We say that $(K, \Sigma)$ (or $\left(K, \Sigma, \Sigma^{\perp}\right)$ ) is gluing if it is full and for each $k<\omega$ there is $n_{0}=n_{0}(k)<\omega$ such that for every $n \geq n_{0}$ and $\left(t_{0}, \ldots, t_{n}\right) \in$ $\operatorname{PFC}(K, \Sigma)$, there is $s \in \Sigma\left(t_{0}, \ldots, t_{n}\right)$ such that

$$
\operatorname{nor}[s] \geq \min \left\{k, \operatorname{nor}\left[t_{0}\right], \ldots, \operatorname{nor}\left[t_{n}\right]\right\} .
$$

3. We say that $\left(K, \Sigma^{\perp}\right)$ (or $\left(K, \Sigma, \Sigma^{\perp}\right)$ ) has the cutting property if for every $t \in K$ with nor $[t]>1$ and an integer $m \in\left(m_{\mathrm{dn}}^{t}, m_{\mathrm{up}}^{t}\right)$, there are $s_{0}, s_{1} \in K$ such that

( $\alpha) m_{\mathrm{dn}}^{s_{0}}=m_{\mathrm{dn}}^{t}, m_{\mathrm{up}}^{s_{0}}=m=m_{\mathrm{dn}}^{s_{1}}, m_{\mathrm{up}}^{s_{1}}=m_{\mathrm{up}}^{t}$,

( $\beta) \operatorname{nor}\left[s_{\ell}\right] \geq \min \left\{\operatorname{nor}[t]-1, m_{\mathrm{dn}}^{t}\right\}$ (for $\left.\ell=0,1\right)$,

$(\gamma)\left\{s_{0}, s_{1}\right\} \in \Sigma^{\perp}(K)$.

Definition 1.1.13. A forcing notion $\mathbb{Q}$ is $\sigma$-n-linked if there is a partition $\left\langle A_{i}\right.$ : $i<\omega\rangle$ of $\mathbb{Q}$ such that

if $q_{0}, \ldots, q_{n-1} \in A_{i}, i \in \omega$ then $(\exists q \in \mathbb{Q})\left(q_{0} \leq q \& \ldots \& q_{n-1} \leq q\right)$.

We say that $\mathbb{Q}$ is $\sigma$-*-linked if it is $\sigma$-n-linked for every $n \in \omega$.

Proposition 1.1.14. Let $\mathbf{H}: \omega \longrightarrow \mathcal{H}\left(\omega_{1}\right)$ and let $\left(K, \Sigma, \Sigma^{\perp}\right)$ be a $\otimes$-creating triple for $\mathbf{H}$.

1. If $\left(K, \Sigma, \Sigma^{\perp}\right)$ is linked, gluing and has the cutting property, then the forcing notion $\mathbb{Q}_{\infty}^{*}\left(K, \Sigma, \Sigma^{\perp}\right)$ is $\sigma$-*-linked.

2. If $f: \omega \times \omega \longrightarrow \omega$ is fast and $\left(K, \Sigma, \Sigma^{\perp}\right)$ is local and linked, then the forcing notions $\mathbb{Q}_{\infty}^{*}\left(K, \Sigma, \Sigma^{\perp}\right)$ and $\mathbb{Q}_{f}^{*}\left(K, \Sigma, \Sigma^{\perp}\right)$ are $\sigma$-*-linked.

3. Assume that $h: \omega \times \omega \longrightarrow \omega$ is regressive and $\mathcal{F} \subseteq(\omega \backslash 2)^{\omega}$ is an $h$-closed family which is either countable, or $\geq^{*}$-directed. Suppose $\left(K, \Sigma, \Sigma^{\perp}\right)$ is local and $h$-linked. Then the forcing notion $\mathbb{Q}_{\mathcal{F}}^{*}\left(K, \Sigma, \Sigma^{\perp}\right)$ is $\sigma$-*-linked.

Proof. Straightforward (and the proof of the first part is essentially the same as that of [16, Thm 2.4]; compare the proof of 1.3.4.1). 
1.2. Tree-like conditions. Here we recall the setting of [15, §1.3] and [8]. Since in getting the ccc we will have to require that the tree-creating pair under considerations is local, we will restrict our attention to that case only. So our definitions here are much simpler than those in the general case, but we still try to keep the notation and flavour of the tree case of 115 .

Definition 1.2.1. Let $\mathbf{H}: \omega \longrightarrow \mathcal{H}\left(\omega_{1}\right)$.

1. A local tree-creature for $\mathbf{H}$ is a triple

$$
t=(\text { nor }, \operatorname{val}, \operatorname{dis})=(\operatorname{nor}[t], \operatorname{val}[t], \operatorname{dis}[t])
$$

such that nor $\in \mathbb{R}^{\geq 0}$, dis $\in \mathcal{H}\left(\omega_{1}\right)$, and for some sequence $\eta \in \prod_{i<n} \mathbf{H}(i)$, $n<\omega$, we have

$$
\emptyset \neq \mathbf{v a l} \subseteq\left\{\langle\eta, \nu\rangle: \eta \triangleleft \nu \in \prod_{i \leq n} \mathbf{H}(i)\right\} .
$$

For a tree-creature $t$ we let $\operatorname{pos}(t) \stackrel{\text { def }}{=} \operatorname{rng}(\operatorname{val}[t])$.

The set of all local tree-creatures for $\mathbf{H}$ will be denoted by LTCR $[\mathbf{H}]$, and for $\eta \in \bigcup_{n<\omega} \prod_{i<n} \mathbf{H}(i)$ we let $\operatorname{LTCR}_{\eta}[\mathbf{H}]=\{t \in \operatorname{LTCR}[\mathbf{H}]: \operatorname{dom}(\mathbf{v a l}[t])=\{\eta\}\}$.

2. Let $K \subseteq \operatorname{LTCR}[\mathbf{H}]$. We say that a function $\Sigma: K \longrightarrow \mathcal{P}(K)$ is a local tree composition on $K$ whenever the following conditions are satisfied.

(a) If $t \in \operatorname{LTCR}_{\eta}[\mathbf{H}], \eta \in \prod_{i<n} \mathbf{H}(i), n<\omega$, then $\Sigma(t) \subseteq \operatorname{LTCR}_{\eta}[\mathbf{H}]$.

(b) If $s \in \Sigma(t)$ then $\mathbf{v a l}[s] \subseteq \operatorname{val}[t]$.

(c) [transitivity] If $s \in \Sigma(t)$ then $\Sigma(s) \subseteq \Sigma(t)$.

3. If $K \subseteq \operatorname{LTCR}[\mathbf{H}]$ and $\Sigma$ is a (local) tree composition operation on $K$ then $(K, \Sigma)$ is called a (local) tree-creating pair for $\mathbf{H}$.

Definition 1.2.2. Let $(K, \Sigma)$ be a (local) tree-creating pair for $\mathbf{H}$.

1. We define the forcing notion $\mathbb{Q}_{1}^{\text {tree }}(K, \Sigma)$ as follows.

A condition is a system $p=\left\langle t_{\eta}: \eta \in T\right\rangle$ such that

(a) $T \subseteq \bigcup_{n \in \omega} \prod_{i<n} \mathbf{H}(i)$ is a non-empty tree with $\max (T)=\emptyset$,

(b) $t_{\eta} \in \operatorname{LTCR}_{\eta}[\mathbf{H}] \cap K$ and $\operatorname{pos}\left(t_{\eta}\right)=\operatorname{succ}_{T}(\eta)($ for $\eta \in T$ ),

(c) 1 for every $\eta \in[T]$ we have:

the sequence $\left\langle\operatorname{nor}\left[t_{\eta\lceil k}\right]: \ln (\operatorname{root}(T)) \leq k<\omega\right\rangle$ diverges to infinity.

The order is given by:

$\left\langle t_{\eta}^{1}: \eta \in T^{1}\right\rangle \leq\left\langle t_{\eta}^{2}: \eta \in T^{2}\right\rangle$ if and only if

$T^{2} \subseteq T^{1}$ and $t_{\eta}^{2} \in \Sigma\left(t_{\eta}^{1}\right)$ for each $\eta \in T^{2}$.

If $p=\left\langle t_{\eta}: \eta \in T\right\rangle$, then we write $\operatorname{root}(p)=\operatorname{root}(T), T^{p}=T, t_{\eta}^{p}=t_{\eta}$ etc.

2. Similarly, we define forcing notions $\mathbb{Q}_{\mathcal{F}}^{\text {tree }}(K, \Sigma)$ for a family $\mathcal{F} \subseteq \omega^{\omega}$ and

$\mathbb{Q}_{f}^{\text {tree }}(K, \Sigma)$ for a function $f: \omega \times \omega \longrightarrow \omega$, replacing the condition $(\mathrm{c})_{1}$ by

$(\mathrm{c})_{\mathcal{F}},(\mathrm{c})_{f}$, respectively, where:

(c) $\mathcal{F}(\exists f \in \mathcal{F})(\exists N<\omega)(\forall \eta \in T)\left(\operatorname{lh}(\eta) \geq N \Rightarrow \operatorname{nor}\left[t_{\eta}\right] \geq f(\operatorname{lh}(\eta))\right)$,

$(\mathrm{c})_{f}(\forall n \in \omega)(\exists N<\omega)(\forall \eta \in T)\left(\ln (\eta) \geq N \Rightarrow \operatorname{nor}\left[t_{\eta}\right] \geq f(n, \ln (\eta))\right)$.

3. If $p \in \mathbb{Q}_{x}^{\text {tree }}(K, \Sigma)$ then, for $\eta \in T^{p}$, we let $p^{[\eta]}=\left\langle t_{\nu}^{p}: \nu \in\left(T^{p}\right)^{[\eta]}\right\rangle$.

Definition 1.2.3. Assume that $(K, \Sigma)$ is a tree-creating pair for $\mathbf{H}$. 
1. We say that $(K, \Sigma)$ is linked if for each $\eta \in \bigcup_{n<\omega} \prod_{i<n} \mathbf{H}(i)$ and tree-creatures $t_{0}, t_{1} \in K \cap \operatorname{LTCR}_{\eta}[\mathbf{H}]$ with $\operatorname{nor}\left[t_{0}\right], \operatorname{nor}\left[t_{1}\right]>1$, there is $s \in \Sigma\left(t_{0}\right) \cap \Sigma\left(t_{1}\right)$ such that nor $[s] \geq \min \left\{\operatorname{nor}\left[t_{0}\right], \operatorname{nor}\left[t_{1}\right]\right\}-1$.

2. Let $h: \omega \times \omega \longrightarrow \omega$. The pair $(K, \Sigma)$ is $h$-linked if for each $t_{0}, t_{1} \in K \cap$ $\operatorname{LTCR}_{\eta}[\mathbf{H}]$ such that nor $\left[t_{0}\right], \operatorname{nor}\left[t_{1}\right] \geq k, k>1$, there is $s \in \Sigma\left(t_{0}\right) \cap \Sigma\left(t_{1}\right)$ with nor $[s] \geq h(\operatorname{lh}(\eta), k)$.

Proposition 1.2.4. Let $\mathbf{H}: \omega \longrightarrow \mathcal{H}\left(\omega_{1}\right)$ and let $(K, \Sigma)$ be a local tree-creating pair for $\mathbf{H}$.

1. If $f: \omega \times \omega \longrightarrow \omega$ is fast and $(K, \Sigma)$ is linked, then the forcing notions $\mathbb{Q}_{1}^{\text {tree }}(K, \Sigma)$ and $\mathbb{Q}_{f}^{\text {tree }}(K, \Sigma)$ are $\sigma-*$-linked.

2. Assume that $h: \omega \times \omega \longrightarrow \omega$ is regressive and a family $\mathcal{F} \subseteq(\omega \backslash 2)^{\omega}$ is $h$-closed and either countable, or $\geq^{*}$-directed. Suppose $(K, \Sigma)$ is $h$-linked. Then the forcing notion $\mathbb{Q}_{\mathcal{F}}^{\text {tree }}(K, \Sigma)$ is $\sigma$-*-linked.

Proof. Straightforward.

\subsection{The complexity of our forcing notions.}

Definition 1.3.1. 1. A forcing notion $\left(\mathbb{P}, \leq_{\mathbb{P}}\right)$ is Souslin (Borel, respectively) if $\mathbb{P}, \leq \mathbb{P}$ and the incompatibility relation $\perp_{\mathbb{P}}$ are $\Sigma_{1}^{1}$ (Borel, respectively) subsets of $\mathbb{R}$ and $\mathbb{R} \times \mathbb{R}$.

2. A forcing notion $\left(\mathbb{P}, \leq_{\mathbb{P}}\right.$ ) is very Souslin ccc (very Borel ccc, respectively), if it is Souslin (Borel, resp.), satisfies the ccc and the notion

$$
\text { " }\left\langle r_{n}: n<\omega\right\rangle \text { is a maximal antichain" }
$$

is $\Sigma_{1}^{1}$ (Borel, resp.)

On Souslin forcing notions and their applications see Judah and Shelah [5] and Goldstern and Judah [3] (the results of these two and many other papers on the topic are presented in Bartoszyński and Judah [1]). A systematic treatment of definable forcing notions is presented in [23], [24] (note that very Souslin ccc forcing notions are $\omega-n w-n e p)$. Here we are going to show that the forcing notions built according to the schemes presented above typically are Borel ccc and (sometimes) even very Borel ccc. Thus we have tools for constructing new ccc $\omega$-nw-nep forcing notions (the only examples known before were those coming from random forcing, the Cohen forcing and their FS iterations; see [22, §4] for a discussion of this topic). Note that, by Shelah [26], ccc $\omega$-nw-nep forcing notions cannot add dominating reals. Thus the forcing notions that are covered by 1.4 .4 cannot be represented as very Souslin ccc forcing notions.

Definition 1.3.2. A $\otimes$-creating triple $\left(K, \Sigma, \Sigma^{\perp}\right)$ for $\mathbf{H}$ is regular if the following condition is satisfied.

$(\bullet)$ Assume $\left(w, t_{0}, \ldots, t_{n}\right),\left(u, s_{0}, \ldots, s_{m}\right) \in \mathrm{FC}\left(K, \Sigma, \Sigma^{\perp}\right)$ are such that

- $m_{\mathrm{dn}}^{t_{\ell}}<m_{\mathrm{dn}}^{s_{0}}<m_{\mathrm{up}}^{t_{\ell}} \leq m_{\mathrm{up}}^{s_{0}}$ for some $\ell \leq n$,

- $\operatorname{nor}\left[t_{\ell}\right] \geq 3$ (for the $\ell$ as above), and

- $\left(w, t_{0}, \ldots, t_{n}\right) \leq\left(u, s_{0}, \ldots, s_{m}\right), m_{\mathrm{up}}^{s_{m}} \leq m_{\mathrm{up}}^{t_{n}}$, and $\operatorname{nor}\left[s_{0}\right] \geq 3$.

Then there are $t^{\prime}, t^{\prime \prime}$ such that $\left\{t^{\prime}, t^{\prime \prime}\right\} \in \Sigma^{\perp}\left(t_{\ell}\right), m_{\mathrm{up}}^{t^{\prime}}=m_{\mathrm{dn}}^{s_{0}}=m_{\mathrm{dn}}^{t^{\prime \prime}}, \operatorname{nor}\left[t^{\prime \prime}\right] \geq$ 2 and $u \in \operatorname{pos}\left(w, t_{0}, \ldots, t_{\ell-1}, t^{\prime}\right)$. 
Definition 1.3.3. Let $\mathbf{H}: \omega \longrightarrow \mathcal{H}\left(\omega_{1}\right)$ and let $(K, \Sigma)$ be either a creating pair for $\mathbf{H}$ or a (local) tree-creating pair for $\mathbf{H}$. We say that $(K, \Sigma)$ is really finitary if the following conditions are satisfied:

(a) $\mathbf{H}(n)$ is finite for all $n<\omega$ (so $\mathbf{v a l}[t]$ is finite for all $t \in K$ ), and

(b) for each $n \in \omega$, the set $\left\{t \in K: \operatorname{rng}(\operatorname{val}[t]) \subseteq \prod_{i<n} \mathbf{H}(i)\right\}$ is finite.

Theorem 1.3.4. Let $\mathbf{H}: \omega \longrightarrow \mathcal{H}\left(\omega_{1}\right)$.

1. Let $\left(K, \Sigma, \Sigma^{\perp}\right)$ be a $\otimes$-creating triple for $\mathbf{H}$ such that $K$ is countable.

(a) If $\left(K, \Sigma, \Sigma^{\perp}\right)$ is regular, linked, gluing and has the cutting property, then the forcing notion $\mathbb{Q}_{\infty}^{*}\left(K, \Sigma, \Sigma^{\perp}\right)$ is Souslin ccc.

(b) If $f: \omega \times \omega \longrightarrow \omega$ is fast and $\left(K, \Sigma, \Sigma^{\perp}\right)$ is local and linked then $\mathbb{Q}_{\infty}^{*}\left(K, \Sigma, \Sigma^{\perp}\right)$ and $\mathbb{Q}_{f}^{*}\left(K, \Sigma, \Sigma^{\perp}\right)$ are Borel ccc.

(c) Assume that $h: \omega \times \omega \longrightarrow \omega$ is a regressive function and $\mathcal{F} \subseteq(\omega \backslash 2)^{\omega}$ is a countable $h$-closed family which is $\geq^{*}$-directed. If $\left(K, \Sigma, \Sigma^{\perp}\right)$ is local and $h$-linked, then $\mathbb{Q}_{\mathcal{F}}^{*}\left(K, \Sigma, \Sigma^{\perp}\right)$ is Borel ccc.

2. Assume that $(K, \Sigma)$ is a local tree-creating pair for $\mathbf{H}$ and $K$ is countable.

(a) If $f: \omega \times \omega \longrightarrow \omega$ is fast and $(K, \Sigma)$ is linked, then $\mathbb{Q}_{f}^{\text {tree }}(K, \Sigma)$ is Borel ccc.

(b) Suppose that $h: \omega \times \omega \longrightarrow \omega$ is regressive and $\mathcal{F} \subseteq(\omega \backslash 2)^{\omega}$ is a countable $h$-closed family which is $\geq^{*}$-directed. If $(K, \Sigma)$ is $h$-linked, then $\mathbb{Q}_{\mathcal{F}}^{\text {tree }}(K, \Sigma)$ is Borel ccc.

3. If in 1(c) and 2(b) above the pair $(K, \Sigma)$ is really finitary, then the respective forcing notions are very Borel ccc.

Proof. 1(a) Let $\mathcal{X}=\left(\bigcup_{n<\omega} \prod_{i<n} \mathbf{H}(i)\right) \times K^{\omega}$ be equipped with the product topology (of countably many countable discrete spaces). So $\mathcal{X}$ is a Polish space and it should be clear that $\mathbb{Q}_{\emptyset}^{*}\left(K, \Sigma, \Sigma^{\perp}\right), \mathbb{Q}_{\infty}^{*}\left(K, \Sigma, \Sigma^{\perp}\right)$ are its Borel subsets. To express " $p \leq q$ " we have to say that there is a sequence $p=p_{0}, \ldots, p_{n}=q$ of elements of $\mathbb{Q}_{\emptyset}^{*}\left(K, \Sigma, \Sigma^{\perp}\right)$ such that $p_{i+1}$ is obtained from $p_{i}$ by one of the operations described in 1.1.3. Each of these operations corresponds to a Borel subset of $\mathcal{X} \times \mathcal{X}$, so easily $\leq_{\mathbb{Q}_{\emptyset}^{*}\left(K, \Sigma, \Sigma^{\perp}\right)}, \leq_{\mathbb{Q}_{\infty}^{*}\left(K, \Sigma, \Sigma^{\perp}\right)}$ are $\Sigma_{1}^{1}$ subsets of $\mathcal{X} \times \mathcal{X}$. The main difficulty is to show that the incompatibility relation $\perp_{\mathbb{Q}_{\infty}^{*}\left(K, \Sigma, \Sigma^{\perp}\right)}$ is a $\Sigma_{1}^{1}$ subset of $\mathcal{X} \times \mathcal{X}$. But this follows from the following observation (note that this is the place where we use the assumption that $\left(K, \Sigma, \Sigma^{\perp}\right)$ is regular).

Claim 1.3.4.1. Conditions $p, q \in \mathbb{Q}_{\infty}^{*}\left(K, \Sigma, \Sigma^{\perp}\right)$ are compatible if and only if there are $N, \ell, m<\omega, t_{0}^{\prime}, t_{1}^{\prime}, t_{0}^{\prime \prime}, t_{1}^{\prime \prime} \in K$ and $u$ such that

- $m_{\mathrm{dn}}^{t_{e}^{p}} \leq N<m_{\mathrm{up}}^{t_{e}^{p}}, \quad m_{\mathrm{dn}}^{t_{m}^{q}} \leq N<m_{\mathrm{up}}^{t_{m}^{q}}$,

- $\left\{t_{0}^{\prime}, t_{1}^{\prime}\right\} \in \Sigma^{\perp}\left(t_{\ell}^{p}\right), \quad\left\{t_{0}^{\prime \prime}, t_{1}^{\prime \prime}\right\} \in \Sigma^{\perp}\left(t_{m}^{q}\right), \quad m_{\mathrm{dn}}^{t_{1}^{\prime}}=m_{\mathrm{dn}}^{t_{1}^{\prime \prime}}=N$,

- $\operatorname{nor}\left[t_{1}^{\prime}\right] \geq 2, \quad \operatorname{nor}\left[t_{1}^{\prime \prime}\right] \geq 2$,

- $u \in \operatorname{pos}\left(w^{p}, t_{0}^{p}, \ldots, t_{\ell-1}^{p}, t_{0}^{\prime}\right) \cap \operatorname{pos}\left(w^{q}, t_{0}^{q}, \ldots, t_{m-1}^{q}, t_{0}^{\prime \prime}\right)$,

- $(\forall n>\ell)\left(\operatorname{nor}\left[t_{n}^{p}\right] \geq 2\right)$ and $(\forall n>m)\left(\operatorname{nor}\left[t_{n}^{q}\right] \geq 2\right)$.

(If $N=m_{\mathrm{dn}}^{t_{\ell}^{p}}$ then $t_{0}^{\prime}$ is not present; similarly on the $q$ side.)

Proof of the claim. First assume that conditions $p, q \in \mathbb{Q}_{\infty}^{*}\left(K, \Sigma, \Sigma^{\perp}\right)$ are compatible and let $r \in \mathbb{Q}_{\infty}^{*}\left(K, \Sigma, \Sigma^{\perp}\right)$ be stronger than both $p$ and $q$. Passing to a stronger condition we may demand that if $\ell, m$ are such that

$$
m_{\mathrm{dn}}^{t_{\ell}^{p}} \leq m_{\mathrm{dn}}^{t_{0}^{r}}<m_{\mathrm{up}}^{t_{e}^{p}}, \quad m_{\mathrm{dn}}^{t_{m}^{q}} \leq m_{\mathrm{dn}}^{t_{0}^{r}}<m_{\mathrm{up}}^{t_{m}^{q}}
$$


then $m_{\mathrm{up}}^{t_{\ell}^{p}} \leq m_{\mathrm{up}}^{t_{0}^{r}}, m_{\mathrm{up}}^{t_{m}^{q}} \leq m_{\mathrm{up}}^{t_{0}^{r}}$ and that nor $\left[t_{0}^{r}\right] \geq 5$ and

$$
m_{\mathrm{up}}^{t_{n}^{p}} \geq \operatorname{lh}\left(w^{r}\right) \quad \Rightarrow \quad \operatorname{nor}\left[t_{n}^{p}\right] \geq 5, \quad \text { and } \quad m_{\mathrm{up}}^{t_{n}^{q}} \geq \operatorname{lh}\left(w^{r}\right) \quad \Rightarrow \quad \operatorname{nor}\left[t_{n}^{q}\right] \geq 5 .
$$

Now we may apply the regularity of $\left(K, \Sigma, \Sigma^{\perp}\right)$ (see 1.3.2) to get $t_{0}^{\prime}, t_{1}^{\prime}, t_{0}^{\prime \prime}, t_{1}^{\prime \prime} \in K$ such that

$$
\begin{aligned}
& \left\{t_{0}^{\prime}, t_{1}^{\prime}\right\} \in \Sigma^{\perp}\left(t_{\ell}^{p}\right), \quad\left\{t_{0}^{\prime \prime}, t_{1}^{\prime \prime}\right\} \in \Sigma^{\perp}\left(t_{m}^{q}\right), \quad \operatorname{nor}\left[t_{1}^{\prime}\right] \geq 2, \quad \operatorname{nor}\left[t_{1}^{\prime \prime}\right] \geq 2 \quad \text { and } \\
& u=w^{r} \in \operatorname{pos}\left(w^{p}, t_{0}^{p}, \ldots, t_{\ell-1}^{p}, t_{0}^{\prime}\right) \cap \operatorname{pos}\left(w^{q}, t_{0}^{q}, \ldots, t_{m-1}^{q}, t_{0}^{\prime \prime}\right) .
\end{aligned}
$$

Put $N=\ln \left(w^{r}\right)$ and check that all demands are satisfied.

For the other implication suppose that $N, \ell, m, t_{0}^{\prime}, t_{1}^{\prime}, t_{0}^{\prime \prime}, t_{1}^{\prime \prime}$ and $u$ are as in the second statement. Choose increasing sequences $\left\langle n_{i}: i<\omega\right\rangle$ and $\left\langle k_{i}: i<\omega\right\rangle$ such that $n_{0}>\ell+5, k_{0}>m+5$ and

- $\left(\forall n \geq n_{i}\right)\left(\operatorname{nor}\left[t_{n}^{p}\right] \geq i+5\right)$ and $\left(\forall n \geq k_{i}\right)\left(\operatorname{nor}\left[t_{n}^{q}\right] \geq i+5\right), \quad$ and

- $m_{\mathrm{dn}}^{t_{k_{i}}^{q}} \leq m_{\mathrm{dn}}^{t_{n_{i}}^{p}}<m_{\mathrm{up}}^{t_{k_{i}}^{q}}, \quad m_{\mathrm{up}}^{t_{k_{i}+1}^{q}}<m_{\mathrm{dn}}^{t_{n_{i+1}}^{p}}$.

Apply the cutting property to choose (for each $i<\omega) s_{i}^{\prime}, s_{i}^{\prime \prime} \in K$ such that

$$
\left\{s_{i}^{\prime}, s_{i}^{\prime \prime}\right\} \in \Sigma^{\perp}\left(t_{k_{i}}^{q}\right), \quad m_{\mathrm{dn}}^{s_{i}^{\prime}}=m_{\mathrm{dn}}^{t_{k_{i}}^{q}}, \quad m_{\mathrm{dn}}^{s_{i}^{\prime \prime}}=m_{\mathrm{dn}}^{t_{n_{i}}^{p}}, \quad \text { and } \quad \operatorname{nor}\left[s_{i}^{\prime}\right], \operatorname{nor}\left[s_{i}^{\prime \prime}\right] \geq i+4 .
$$

(If $m_{\mathrm{dn}}^{t_{n_{i}}^{p}}=m_{\mathrm{dn}}^{t_{k_{i}}^{q}}$ then the $s_{i}^{\prime}$ is not present.) Next use gluing to choose $r_{i}, s_{i}$ so that

$$
\begin{array}{ll}
r_{0} \in \Sigma\left(t_{1}^{\prime}, t_{\ell+1}^{p}, \ldots, t_{n_{0}-1}^{p}\right), & s_{0} \in \Sigma\left(t_{1}^{\prime \prime}, t_{m+1}^{q}, \ldots, t_{k_{0}-1}^{q}, s_{0}^{\prime}\right) \\
r_{i+1} \in \Sigma\left(t_{n_{i}}^{p}, \ldots, t_{n_{i+1}-1}^{p}\right), & s_{i+1} \in \Sigma\left(s_{i}^{\prime \prime}, t_{k_{i}+1}^{q}, \ldots, t_{k_{i+1}-1}^{q}, s_{i+1}^{\prime}\right), \\
\operatorname{nor}\left[r_{i}\right], \operatorname{nor}\left[s_{i}\right] \geq i+2 . &
\end{array}
$$

Since $(K, \Sigma)$ is linked we may choose $t_{i} \in \Sigma\left(r_{i}\right) \cap \Sigma\left(s_{i}\right)$ such that $\operatorname{nor}\left[t_{i}\right] \geq i+1$. Now look at $\left(u, t_{0}, t_{1}, \ldots\right)$. It is a condition in $\mathbb{Q}_{\infty}^{*}\left(K, \Sigma, \Sigma^{\perp}\right)$ stronger than both $p$ and $q$.

1(b,c) and 2(a,b) Similarly (and much easier).

3. Let $h \in \omega^{\omega}$ be a regressive function and let $\mathcal{F} \subseteq(\omega \backslash 2)^{\omega}$ be a countable $h$-closed family which is $\geq^{*}$-directed. Suppose that $(K, \Sigma)$ is a local, $h$-linked and really finitary creating pair (because of the "local" $\Sigma^{\perp}$ can be omitted as it is trivial). We are going to show that "being a (countable) pre-dense subset of $\mathbb{Q}_{\mathcal{F}}^{*}(K, \Sigma)$ " is a Borel property.

Let $\mathcal{X}=\left(\bigcup_{n<\omega} \prod_{i<n} \mathbf{H}(i)\right) \times K^{\omega}, \mathcal{X}^{\omega}$ and $\mathcal{Y}=\mathcal{P}(\mathrm{FC}(K, \Sigma))$ be equipped with the natural (product) Polish topologies (note that $\mathrm{FC}(K, \Sigma)$ is a countable set). For $\bar{p}=\left\langle p_{n}: n<\omega\right\rangle \in \mathcal{X}^{\omega}, \bar{p} \subseteq \mathbb{Q}_{\mathcal{F}}^{*}(K, \Sigma), w \in \bigcup_{m<\omega} \prod_{i<m} \mathbf{H}(i)$ and $f \in \mathcal{F}$ we define

$$
N^{\bar{p}}(n)=\min \left\{m_{\mathrm{dn}}^{t_{i}^{p_{n}}}:(\forall j \geq i)\left(\operatorname{nor}\left[t_{j}^{p_{n}}\right] \geq 2\right)\right\}
$$

and

$$
\begin{aligned}
& \mathcal{T}_{w, f}^{\bar{p}}=\quad\left\{\left(w, t_{0}, \ldots, t_{k}\right) \in \mathrm{FC}(K, \Sigma):(\forall i \leq k)\left(\operatorname{nor}\left[t_{i}\right] \geq f\left(m_{\mathrm{dn}}^{t_{i}}\right)\right)\right. \text { and } \\
&\left.(\forall n<\omega)\left(N^{\bar{p}}(n) \leq m_{\mathrm{up}}^{t_{k}} \Rightarrow \operatorname{pos}\left(w, t_{0}, \ldots, t_{k}\right) \cap \operatorname{POS}\left(p_{n}\right)=\emptyset\right)\right\} .
\end{aligned}
$$

Note that $\left(\mathbb{Q}_{\mathcal{F}}^{*}(K, \Sigma)\right)^{\omega}$ is a Borel subset of $\mathcal{X}^{\omega}$ and the functions

$$
\bar{p} \mapsto N^{\bar{p}}:\left(\mathbb{Q}_{\mathcal{F}}^{*}(K, \Sigma)\right)^{\omega} \longrightarrow \omega^{\omega} \quad \text { and } \quad \bar{p} \mapsto \mathcal{T}_{w, f}^{\bar{p}}:\left(\mathbb{Q}_{\mathcal{F}}^{*}(K, \Sigma)\right)^{\omega} \longrightarrow \mathcal{Y}
$$

are Borel. Now, each $\mathcal{T}_{w, f}^{\bar{p}}$ is essentially a finitary tree, so

$$
\mathcal{T}_{w, f}^{\bar{p}} \text { is well founded if and only if } \quad \mathcal{T}_{w, f}^{\bar{p}} \text { is finite. }
$$


Consequently, for each $w$ and $f$, the set

$$
\left\{\bar{p} \in\left(\mathbb{Q}_{\mathcal{F}}^{*}(K, \Sigma)\right)^{\omega}: \mathcal{T}_{w, f}^{\bar{p}} \text { is well founded }\right\}
$$

is Borel. Since there are countably many possibilities for $w$ and $f$, we easily finish the proof using the following observation.

Claim 1.3.4.2. Let $\bar{p}=\left\langle p_{n}: n\langle\omega\rangle \in\left(\mathbb{Q}_{\mathcal{F}}^{*}(K, \Sigma)\right)^{\omega}\right.$. Then $\bar{p}$ is pre-dense in $\mathbb{Q}_{\mathcal{F}}^{*}(K, \Sigma)$ if and only if for each $w \in \bigcup_{m<\omega} \prod_{i<m} \mathbf{H}(i)$ and $f \in \mathcal{F}$ the tree $\mathcal{T}_{w, f}^{\bar{p}}$ is well-founded.

Proof of the claim. Suppose that, for some $w$ and $f$, the tree $\mathcal{T}_{w, f}^{\bar{p}}$ has an $\omega$-branch and let $q=\left(w, t_{0}, t_{1}, \ldots\right)$ be such a branch. Necessarily $q \in \mathbb{Q}_{\mathcal{F}}^{*}(K, \Sigma)$ (as witnessed by $f)$. If follows from the definition of $\mathcal{T}_{w, f}^{\bar{p}}$ that $\operatorname{POS}(q) \cap \operatorname{POS}\left(p_{n}\right)$ is finite for each $n \in \omega$ and therefore $q \perp_{\mathbb{Q}_{\mathcal{F}}^{*}(K, \Sigma)} p_{n}$ (remember 1.1.11).

Now assume that $\bar{p}$ is not pre-dense in $\mathbb{Q}_{\mathcal{F}}^{*}(K, \Sigma)$ and let $q \in \mathbb{Q}_{\mathcal{F}}^{*}(K, \Sigma)$ be a condition incompatible with all $p_{n}$. We may demand that for some $f \in \mathcal{F}$ we have $(\forall i \in \omega)\left(\operatorname{nor}\left[t_{i}^{q}\right] \geq f\left(m_{\mathrm{dn}}^{t_{i}^{q}}\right)\right)$. It should be clear that $q$ determines an $\omega$-branch in the tree $\mathcal{T}_{w^{q}, f}^{\bar{p}}$ (remember that $(K, \Sigma)$ is $h$-linked).

Similarly we deal with the respective variant of 2(b).

\subsection{Unbounded and dominating reals.}

Lemma 1.4.1. Let $\mathbf{V} \subseteq \mathbf{V}^{*}$ be universes of $\mathrm{ZFC}^{*}$. Assume that $\left\langle f_{i}: i<\omega\right\rangle \in \mathbf{V}$ and $g \in \mathbf{V}^{*}$ are such that

(a) $g \in \omega^{\omega}, f_{i} \in \omega^{\omega}, f_{i+1}<^{*} f_{i}$ for all $i \in \omega$,

(b) $(\forall i \in \omega)\left(\exists^{\infty} k \in \omega\right)\left(g(k)<f_{i}(k)\right)$,

(c) if $h \in \omega^{\omega} \cap \mathbf{V}$ is such that $(\forall i \in \omega)\left(h<^{*} f_{i}\right)$, then $h<^{*} g$.

Then $\omega^{\omega} \cap \mathbf{V}$ is bounded in $\mathbf{V}^{*}$.

Proof. It follows from the assumptions (a), (b) that we may find an infinite set $K=\left\{k_{0}, k_{1}, k_{2}, \ldots\right\} \in \mathbf{V}^{*} \cap[\omega]^{\omega}$ such that for each $i \in \omega$ we have

(*) $f_{0}\left(k_{i}\right)>f_{1}\left(k_{i}\right)>\ldots>f_{i}\left(k_{i}\right)>g\left(k_{i}\right)$.

Let $\varphi \in \omega^{\omega} \cap \mathbf{V}^{*}$ be such that $(\forall n \in \omega)\left(|K \cap(n, \varphi(n))|>2^{n+1}\right)$. We claim that the function $\varphi$ dominates $\omega^{\omega} \cap \mathbf{V}$, i.e.

$$
\left(\forall f \in \omega^{\omega} \cap \mathbf{V}\right)\left(\forall^{\infty} n \in \omega\right)(f(n)<\varphi(n)) .
$$

If not, then we may choose an increasing sequence $\left\langle n_{i}: i<\omega\right\rangle \in \mathbf{V}$ of integers such that $n_{0}=0$ and

(i) $\left(\exists^{\infty} i \in \omega\right)\left(\left|\left(n_{i}, n_{i+1}\right) \cap K\right|>2^{n_{i}}\right)$,

(ii) $(\forall i \in \omega)\left(\forall n \geq n_{i+1}\right)\left(f_{i+1}(n)<f_{i}(n)\right)$.

Define $h \in \omega^{\omega} \cap \mathbf{V}$ by $h \uparrow\left[n_{i}, n_{i+1}\right)=f_{i} \uparrow\left[n_{i}, n_{i+1}\right.$ ) (for $i \in \omega$ ). It follows from (ii) that $h<^{*} f_{i}$ for each $i \in \omega$, so we may apply the assumption (c) to conclude that $h<^{*} g$. But look at the clauses $(*)$ and (i) above. Whenever $\left|\left(n_{i}, n_{i+1}\right) \cap K\right|>2^{n_{i}}$, there is $\ell \in \omega$ such that $k_{\ell} \in\left(n_{i}, n_{i+1}\right), \ell>i$ and

$$
f_{0}\left(k_{\ell}\right)>\ldots>f_{i}\left(k_{\ell}\right)=h\left(k_{\ell}\right)>\ldots>f_{\ell}\left(k_{\ell}\right)>g\left(k_{\ell}\right),
$$

so easily we get a contradiction.

Definition 1.4.2. Let $(K, \Sigma)$ be a creating pair or a (local) tree-creating pair. 
1. (See 15 , Def. 5.1.6]) We say that $(K, \Sigma)$ is reducible if for each $t \in K$ with nor $[t] \geq 3$, there is $s \in \Sigma(t)$ such that $\frac{\operatorname{nor}[t]}{2} \leq \operatorname{nor}[s] \leq \operatorname{nor}[t]-1$.

2. The pair $(K, \Sigma)$ is normal if it is reducible, linked and if

$(\boxplus)$ for each $s, t \in K$ :

$$
\operatorname{nor}[s]<\operatorname{nor}[t] \quad \Rightarrow \quad(\forall u \in \operatorname{dom}(\operatorname{val}[t]))(\exists v)(\langle u, v\rangle \in \operatorname{val}[t] \backslash \operatorname{val}[s]) \text {. }
$$

3. A creating pair $(K, \Sigma)$ is semi-normal if it is linked, and for each $n \in \omega$ and $t \in K$ such that nor $[t]>2^{n+2}, m_{\mathrm{up}}^{t}-m_{\mathrm{dn}}^{t}>2^{2^{n+4}}$, there is a sequence $\left\langle s_{\ell}: \ell \leq n\right\rangle \subseteq K$ satisfying

( $\alpha) s_{0}=t, s_{\ell+1} \in \Sigma\left(s_{\ell}\right), 2^{n+1-\ell}<\operatorname{nor}\left[s_{\ell+1}\right] \leq 2^{n-\ell+2}$ (for $\ell<n$ ), and

( $\beta)$ if $s \in K, m_{\mathrm{dn}}^{s}=m_{\mathrm{dn}}^{t}, m_{\mathrm{up}}^{s}=m_{\mathrm{up}}^{t}$ and nor $[s]>2^{n-\ell+3}, \ell<n$, then

$$
(\forall u \in \operatorname{dom}(\operatorname{val}[s]))(\exists v)\left(\langle u, v\rangle \in \operatorname{val}[s] \backslash \operatorname{val}\left[s_{\ell+1}\right]\right) .
$$

4. A $\otimes$-creating triple $\left(K, \Sigma, \Sigma^{\perp}\right)$ (or just $\left.(K, \Sigma)\right)$ is super-gluing if it is gluing and for every $s_{0}, \ldots, s_{k} \in K$ and $N \in \omega$ such that $m_{\mathrm{up}}^{s_{i}} \leq m_{\mathrm{dn}}^{s_{i+1}}$ for $i<k$ and $m_{\mathrm{up}}^{s_{k}} \leq N$, there is $s \in K$ satisfying:

- $m_{\mathrm{dn}}^{s}=m_{\mathrm{dn}}^{s_{0}}, m_{\mathrm{up}}^{s}=N, \operatorname{dom}(\operatorname{val}[s])=\operatorname{dom}\left(\operatorname{val}\left[s_{0}\right]\right)$, and

- $\operatorname{nor}[s] \geq \min \left\{\operatorname{nor}\left[s_{i}\right]: i \leq k\right\}-1$, and

- $(\forall\langle u, v\rangle \in \operatorname{val}[s])(\forall i \leq k)\left(\left\langle v\left\lceil m_{\mathrm{dn}}^{s_{i}}, v\left\lceil m_{\mathrm{up}}^{s_{i}}\right\rangle \in \operatorname{val}\left[s_{i}\right]\right)\right.\right.$.

Remark 1.4.3. Note that "normal" implies "semi-normal". What we really need in the proofs of 1.4.4 $(1,2)$ is semi-normality (or rather a suitable variant of it). However, the normality is more natural and only in the case of $\otimes$-creating triples (which are gluing and have the cutting property) the natural norms are semi-normal but not normal; see Examples at the end of this section.

Theorem 1.4.4. 1. Let $f: \omega \times \omega \longrightarrow \omega$ be a fast function, and let $(K, \Sigma)$ be a local creating pair (a local tree-creating pair, respectively). Assume that $(K, \Sigma)$ is normal and $\mathbb{Q}_{f}^{*}(K, \Sigma) \neq \emptyset\left(\mathbb{Q}_{f}^{\text {tree }}(K, \Sigma) \neq \emptyset\right.$, resp. $)$. Then the forcing notion $\mathbb{Q}_{f}^{*}(K, \Sigma)\left(\mathbb{Q}_{f}^{\text {tree }}(K, \Sigma)\right.$, resp. $)$ adds a dominating real.

2. If $(K, \Sigma)$ is a normal (local) tree-creating pair and $\mathbb{Q}_{1}^{\text {tree }}(K, \Sigma) \neq \emptyset$, then the forcing notion $\mathbb{Q}_{1}^{\text {tree }}(K, \Sigma) \neq \emptyset$ adds a dominating real.

3. Assume that $\left(K, \Sigma, \Sigma^{\perp}\right)$ is a semi-normal $\otimes$-creating triple which is supergluing and has the cutting property (and $\mathbb{Q}_{\infty}^{*}\left(K, \Sigma, \Sigma^{\perp}\right) \neq \emptyset$ ). Then the forcing notion $\mathbb{Q}_{\infty}^{*}\left(K, \Sigma, \Sigma^{\perp}\right)$ adds a dominating real.

Proof. In all cases we will use Lemma 1.4 .1 for functions $f_{i} \in \prod_{n \in \omega}(n+1)$ defined by $f_{i} \uparrow[0, i) \equiv 0, f_{i}(n)=n-i$ for $n \geq i$ (for $i \in \omega$ ) and a suitably chosen name $\dot{g}$ for a function in $\prod_{n \in \omega}(n+1)$.

(1) We consider the case when $(K, \Sigma)$ is a local creating pair only.

Let $p \in \mathbb{Q}_{f}^{*}(K, \Sigma)$. Using the normality of $(K, \Sigma)$, choose an increasing sequence $\left\langle m_{n}: n<\omega\right\rangle \subseteq \omega$ and a sequence $\left\langle s_{n}^{\ell}: \ell \leq n, n<\omega\right\rangle \subseteq K$ such that for each $n \in \omega$ and $\ell<n$ :

(a) $s_{n}^{0}=t_{m_{n}}^{p}, \operatorname{nor}\left[t_{m_{n}}^{p}\right]>f\left(n+2, m_{\mathrm{dn}}^{t_{m_{n}}^{p}}\right)$,

(b) $s_{n}^{\ell+1} \in \Sigma\left(s_{n}^{\ell}\right), f\left(n-\ell+1, m_{\mathrm{dn}}^{s_{n}^{\ell+1}}\right)<\operatorname{nor}\left[s_{n}^{\ell+1}\right] \leq f\left(n-\ell+2, m_{\mathrm{dn}}^{s_{n}^{\ell+1}}\right)$.

Let $\dot{W}$ be the name for $\mathbb{Q}_{f}^{*}(K, \Sigma)$-generic real, i.e.

$$
\Vdash_{\mathbb{Q}_{f}^{*}(K, \Sigma)} \dot{W}=\bigcup\left\{w^{q}: q \in \Gamma_{\mathbb{Q}_{f}^{*}(K, \Sigma)}\right\}
$$


(see [15, Def. 1.1.13, Prop. 1.1.14]). Let $\dot{g}$ be a $\mathbb{Q}_{f}^{*}(K, \Sigma)$-name for a function in $\prod_{n \in \omega}(n+1)$ defined by

$$
p \Vdash "(\forall n \in \omega)(\forall \ell \leq n)\left(\dot{g}(n)=\ell \Leftrightarrow\left\langle\dot{W}\left\lceil m_{\mathrm{dn}}^{s_{n}^{\ell}}, \dot{W}\left\lceil m_{\mathrm{up}}^{s_{n}^{\ell}}\right\rangle \in \operatorname{val}\left[s_{n}^{\ell}\right] \backslash \operatorname{val}\left[s_{n}^{\ell+1}\right]\right) "\right.\right.
$$

(if $\left\langle\dot{W}\left\lceil m_{\mathrm{dn}}^{s_{n}^{n}}, \dot{W}\left\lceil m_{\mathrm{up}}^{s_{n}^{n}}\right\rangle \in \operatorname{val}\left[s_{n}^{n}\right]\right.\right.$ then $\left.\dot{g}(n)=n\right)$.

Claim 1.4.4.1. ( $(\alpha) p \Vdash_{\mathbb{Q}_{f}^{*}(K, \Sigma)}(\forall i \in \omega)\left(\exists^{\infty} n \in \omega\right)\left(\dot{g}(n)<f_{i}(n)\right)$,

( $\beta$ ) Assume that $h \in \omega^{\omega}$ is such that $h<^{*} f_{i}$ for all $i \in \omega$. Then $p \Vdash$ " $h<^{*} \dot{g}$ ".

Proof of the claim. ( $\alpha)$ Let $i \in \omega$ and let $p_{0} \geq p, N \in \omega$. Take $n>N+i$ so that for some $k<\omega$ we have $t_{k}^{p_{0}} \in \Sigma\left(t_{m_{n}}^{p}\right)$ and nor $\left[t_{k}^{p_{0}}\right]>f\left(i+3, m_{\mathrm{dn}}^{t_{k}^{p_{0}}}\right)$. Since $(K, \Sigma)$ is normal, we may find $w \in \operatorname{pos}\left(w^{p_{0}}, t_{0}^{p_{0}}, \ldots, t_{k}^{p_{0}}\right)$ such that $\left\langle w\left\lceil m_{\mathrm{dn}}^{t_{k}^{p_{0}}}, w\left\lceil m_{\mathrm{up}}^{t_{k}^{p_{0}}}\right\rangle \notin\right.\right.$ $\operatorname{val}\left[s_{n}^{n-i}\right]$ (remember nor $\left.\left[s_{n}^{n-i}\right] \leq f\left(i+3, m_{\mathrm{dn}}^{s_{n}^{n-i}}\right)\right)$. Clearly the condition $q=$ $\left(w, t_{k+1}^{p_{0}}, t_{k+2}^{p_{0}}, \ldots\right)$ forces that $\dot{g}(n)<n-i=f_{i}(n)$.

( $\beta)$ Let $h \in \omega^{\omega}$ be such that $h<^{*} f_{i}$ for all $i \in \omega$ and let $p_{0} \geq p$. Let $\ell$ be such that $t_{0}^{p_{0}} \in \Sigma\left(t_{\ell}^{p}\right)$ (so $t_{k}^{p_{0}} \in \Sigma\left(t_{k+\ell}^{p}\right)$ for each $k$ ). We may assume that if $m_{n} \geq \ell$ then $h(n)<n-5$ and that $(\forall k<\omega)\left(\operatorname{nor}\left[t_{k}^{p_{0}}\right]>f\left(5, m_{\mathrm{dn}}^{t_{k}^{p_{0}}}\right)\right)$. For each $k \in \omega$ choose $t_{k}$ as follows:

- if $k+\ell \notin\left\{m_{n}: n \in \omega\right\}$, then $t_{k}=t_{k}^{p_{0}}$,

- if $k+\ell=m_{n}, n \in \omega$, then $t_{k} \in \Sigma\left(t_{k}^{p_{0}}\right) \cap \Sigma\left(s_{n}^{h(n)+1}\right)$ is such that $\operatorname{nor}\left[t_{k}\right] \geq$ $\min \left\{\operatorname{nor}\left[t_{k}^{p_{0}}\right], \operatorname{nor}\left[s_{n}^{h(n)+1}\right]\right\}-1$

(remember that $(K, \Sigma)$ is linked). Since $\operatorname{nor}\left[s_{n}^{h(n)+1}\right]>f\left(n-h(n)+1, m_{\mathrm{dn}}^{s_{n}^{0}}\right)$ we easily see that $q=\left(w^{p_{0}}, t_{0}, t_{1}, \ldots\right) \in \mathbb{Q}_{f}^{*}(K, \Sigma)$, and clearly it is a condition stronger than $p_{0}$. As $q \Vdash_{\mathbb{Q}_{f}^{*}(K, \Sigma)}(\forall n \in \omega)\left(m_{n} \geq \ell \Rightarrow \dot{g}(n)>h(n)\right)$, the claim follows.

Now, the first clause of the theorem is an immediate consequence of 1.4.4.1 and 1.4.1.

(2) The proof is similar to the one above. Let $p \in \mathbb{Q}_{1}^{\text {tree }}(K, \Sigma)$. Choose fronts $F_{n}$ of $T^{p}$ (for $n \in \omega$ ) such that for each $n$ :

- $\left(\forall \eta \in F_{n+1}\right)\left(\exists \nu \in F_{n}\right)(\nu \triangleleft \eta)$,

- $\left(\forall \eta \in F_{n}\right)\left(\operatorname{nor}\left[t_{\eta}^{p}\right]>2^{n+2}\right)$

(clearly possible; see [15, Prop. 1.3.8]). For each $n \in \omega$ and $\eta \in F_{n}$ choose a sequence $\left\langle s_{\eta}^{\ell}: \ell \leq n\right\rangle \subseteq K$ such that

$$
s_{\eta}^{0}=t_{\eta}^{p}, \quad s_{\eta}^{\ell+1} \in \Sigma\left(s_{\eta}^{\ell}\right) \quad \text { and } \quad 2^{n-\ell+1}<\operatorname{nor}\left[s_{\eta}^{\ell+1}\right] \leq 2^{n-\ell+2} .
$$

Let $\dot{W}$ be the name for the $\mathbb{Q}_{1}^{\text {tree }}(K, \Sigma)$-generic real and let $\dot{g}$ be a $\mathbb{Q}_{1}^{\text {tree }}(K, \Sigma)$-name for a real in $\prod_{n \in \omega}(n+1)$ such that (the condition $p$ forces that) if $\eta=\dot{W}\left\lceil m \in F_{n}\right.$ (for some $m, n \in \omega)$ and $\dot{W}\left\lceil(m+1) \in \operatorname{pos}\left(s_{\eta}^{\ell}\right) \backslash \operatorname{pos}\left(s_{\eta}^{\ell+1}\right)\right.$, then $\dot{g}(n)=\ell$.

Claim 1.4.4.2. $\quad(\alpha) p \Vdash_{\mathbb{Q}_{1}^{\text {tree }}(K, \Sigma)}(\forall i \in \omega)\left(\exists^{\infty} n \in \omega\right)\left(\dot{g}(n)<f_{i}(n)\right)$,

( $\beta)$ If $h \in \omega^{\omega}$ is such that $h<^{*} f_{i}$ for all $i \in \omega$, then $p \Vdash_{\mathbb{Q}_{1}^{\text {tree }}(K, \Sigma)}$ " $h<^{*} \dot{g}$ ".

Proof of the claim. $(\alpha)$ Like 1.4.4.1 $(\alpha)$.

( $\beta) \quad$ Let $q \geq p$. We may assume that for some $m>2$ we have: $\operatorname{root}(q) \in F_{m}$, $\operatorname{nor}\left[t_{\nu}^{q}\right]>8$ for all $\nu \in T^{q}$, and $h(n)<n-5$ for all $n \geq m$. We build inductively a tree $T \subseteq T^{q}$ and a system $\left\langle t_{\eta}: \eta \in T\right\rangle$ as follows. We declare that $\operatorname{root}(q)=$ 
$\operatorname{root}(T) \in T$. Suppose we have declared that $\eta \in T$. If $\eta \notin \bigcup_{n \in \omega} F_{n}$, then we let $t_{\eta}=t_{\eta}^{q}$ and we declare $\operatorname{pos}\left(t_{\eta}\right) \subseteq T$. If $\eta \in F_{n}$ for some $n \geq m$, then we choose $t_{\eta} \in \Sigma\left(t_{\eta}^{q}\right) \cap \Sigma\left(s_{\eta}^{h(n)+1}\right)$ such that $\operatorname{nor}\left[t_{\eta}\right] \geq \min \left\{\operatorname{nor}\left[t_{\eta}^{q}\right], 2^{n-h(n)+1}\right\}-1$, and we declare $\operatorname{pos}\left(t_{\eta}\right) \subseteq T$.

Finally, we let $q^{*}=\left\langle t_{\eta}: \eta \in T\right\rangle$ and we notice that $q^{*} \Vdash h<^{*} \dot{g}$.

(3) Let $p \in \mathbb{Q}_{\infty}^{*}\left(K, \Sigma, \Sigma^{\perp}\right)$. By "gluing", we may assume that $m_{\mathrm{up}}^{t_{k}^{p}}-m_{\mathrm{dn}}^{t_{k}^{p}}>$ $2^{2^{k+4}}$ for each $k<\omega$. Using semi-normality we may choose an increasing sequence $\left\langle m_{n}: n<\omega\right\rangle$ and a sequence $\left\langle s_{n}^{\ell}: \ell \leq n, n<\omega\right\rangle \subseteq K$ such that

(a) $s_{n}^{0}=t_{m_{n}}^{p}, \operatorname{nor}\left[t_{m_{n}}^{p}\right]>2^{n+2}, s_{n}^{\ell+1} \in \Sigma\left(s_{n}^{\ell}\right), 2^{n-\ell+1}<\operatorname{nor}\left[s_{n}^{\ell+1}\right] \leq 2^{n-\ell+2}$ (for $\ell<n<\omega)$,

(b) if $t \in K, m_{\mathrm{dn}}^{t}=m_{\mathrm{dn}}^{s_{n}^{0}}, m_{\mathrm{up}}^{t}=m_{\mathrm{up}}^{s_{n}^{0}}$, and $\operatorname{nor}[t]>2^{n-\ell+3}, \ell<n<\omega$, then

$$
(\forall u \in \operatorname{dom}(\operatorname{val}[t]))(\exists v)\left(\langle u, v\rangle \in \operatorname{val}[t] \backslash \operatorname{val}\left[s_{n}^{\ell+1}\right]\right) .
$$

Now we define the name $\dot{g}$ like before, so

$$
p \Vdash_{\mathbb{Q}_{\infty}^{*}\left(K, \Sigma, \Sigma^{\perp}\right)} " \dot{g}(n)=\ell \Leftrightarrow\left\langle\dot { W } \left\lceil m_{\mathrm{dn}}^{s_{n}^{\ell}}, \dot{W}\left\lceil m_{\mathrm{up}}^{s_{n}^{\ell}}\right\rangle \in \operatorname{val}\left[s_{n}^{\ell}\right] \backslash \operatorname{val}\left[s_{n}^{\ell+1}\right] " .\right.\right.
$$

Claim 1.4.4.3. ( $(\alpha) p \Vdash_{\mathbb{Q}_{\infty}^{*}\left(K, \Sigma, \Sigma^{\perp}\right)}(\forall i \in \omega)\left(\exists^{\infty} n \in \omega\right)\left(\dot{g}(n)<f_{i}(n)\right)$,

( $\beta)$ If $h<^{*} f_{i}$ for all $i \in \omega$, then $p \Vdash_{\mathbb{Q}_{\infty}^{*}\left(K, \Sigma, \Sigma^{\perp}\right)}$ " $h<^{*} \dot{g}$ ".

Proof of the claim. ( $\alpha$ ) Suppose $q \geq p$, and $i, N<\omega$. Passing to a stronger condition (using "gluing and cutting") we may assume that

- $\operatorname{nor}\left[t_{0}^{q}\right]>2^{i+4}$ and

- $m_{\mathrm{dn}}^{t_{0}^{q}}=m_{\mathrm{dn}}^{t_{m_{n}}^{p}}, m_{\mathrm{up}}^{t_{0}^{q}}=m_{\mathrm{up}}^{t_{m_{n}}^{p}}$ for some $n>N+i+1$.

Choose $w \in \operatorname{pos}\left(w^{q}, t_{0}^{q}\right)$ such that $\left\langle w^{q}, w\right\rangle \notin s_{n}^{n-i}$ and look at the condition $q^{\prime}=$ $\left(w, t_{1}^{q}, t_{2}^{q}, \ldots\right)$.

( $\beta$ ) Let $q \geq p$. Passing to a stronger condition if necessary, we may assume that for some increasing sequence $\left\langle N_{k}: k<\omega\right\rangle \subseteq \omega$ we have:

- $m_{\mathrm{dn}}^{t_{k}^{q}}=m_{\mathrm{dn}}^{t_{m_{N_{k}}}^{p}}, m_{\mathrm{up}}^{t_{k}^{q}}=m_{\mathrm{dn}}^{t_{m_{k+1}}^{p}}, \operatorname{nor}\left[t_{k}^{q}\right]>5$ for all $k<\omega$,

- if $n \geq N_{0}$ then $h(n)<n-5$.

Using "super-gluing" choose creatures $s_{k} \in K$ (for $k \in \omega$ ) such that

- $m_{\mathrm{dn}}^{s_{k}}=m_{\mathrm{dn}}^{t_{m_{N_{k}}}^{p}}, \quad m_{\mathrm{up}}^{s_{k}}=m_{\mathrm{dn}}^{t_{m_{N+1}}^{p}}, \quad$ and

- $\operatorname{nor}\left[s_{k}\right] \geq \min \left\{\operatorname{nor}\left[s_{n}^{h(n)+1}\right]: N_{k} \leq n<N_{k+1}\right\}-1$, and

- $\left(\forall\langle u, v\rangle \in \operatorname{val}\left[s_{k}\right]\right)\left(\forall n \in\left[N_{k}, N_{k+1}\right)\right)\left(\left\langle v\left\lceil m_{\mathrm{dn}}^{s_{n}^{0}}, v\left\lceil m_{\mathrm{up}}^{s_{n}^{0}}\right\rangle \in \operatorname{val}\left[s_{n}^{h(n)+1}\right]\right)\right.\right.$.

Apply "linked" to choose creatures $t_{k} \in \Sigma\left(s_{k}\right) \cap \Sigma\left(t_{k}^{q}\right)$ such that

$$
1+\operatorname{nor}\left[t_{k}\right] \geq \min \left\{\operatorname{nor}\left[s_{k}\right], \operatorname{nor}\left[t_{k}^{q}\right]\right\} \geq \min \left\{\operatorname{nor}\left[t_{k}^{q}\right], 2^{n-h(n)}: N_{k} \leq n<N_{k+1}\right\} .
$$

Then $q^{*}=\left(w^{q}, s_{0}, s_{1}, s_{2}, \ldots\right)$ is a condition in $\mathbb{Q}_{\infty}^{*}\left(K, \Sigma, \Sigma^{\perp}\right), q^{*} \geq q$ and it forces that $\left(\forall n \geq N_{0}\right)(h(n)<\dot{g}(n))$.

Remark 1.4.5. Note that $1.4 .4(1)$ applies to forcing notions $\mathbb{Q}_{\infty}^{*}(K, \Sigma)$ too, see 1.1.7. 
Definition 1.4.6. A ccc forcing notion $\mathbb{P}$ is nice if there is a partition $\left\langle P_{m}: m<\omega\right\rangle$ of $\mathbb{P}$ such that

$(\star)$ if $\left\langle p_{n}: n<\omega\right\rangle \subseteq \mathbb{P}$ is a maximal antichain in $\mathbb{P}, m \in \omega$, then there is $N<\omega$ such that

$$
\left(\forall p \in P_{m}\right)(\exists n<N)\left(p, p_{n} \text { are compatible }\right) .
$$

Theorem 1.4.7 (Miller [12, Brendle and Judah [2]; see [1, Thm. 6.5.11]). If $\mathcal{F} \subseteq$ $\omega^{\omega}$ is an unbounded family and $\mathbb{P}$ is a nice ccc forcing notion, then

$$
\Vdash_{\mathbb{P}} \text { " the family } \mathcal{F} \text { is unbounded ". }
$$

Remark 1.4.8. Since no dominating reals can be added at limit stages of FS iterations of ccc forcing notions (see [28, Con. VI.3.17]), it follows from 1.4.7 that FS iterations of nice forcing notions do not add dominating reals.

Definition 1.4.9. $\quad 1$. Let $(K, \Sigma)$ be a local creating pair (or a local tree-creating pair) for $\mathbf{H}$. We say that $(K, \Sigma)$ is Cohen-producing if for each $n \in \omega$ there is a set $A_{n} \subseteq \mathbf{H}(n)$ such that

if $t \in K, \operatorname{nor}[t]>1, u \in \operatorname{dom}(\operatorname{val}[t]), \operatorname{lh}(u)=n$, then there are $v_{0}, v_{1}$ such that $\left\langle u, v_{0}\right\rangle,\left\langle u, v_{1}\right\rangle \in \operatorname{val}[t]$ and $v_{1}(n) \in A_{n}$ and $v_{0}(n) \notin A_{n}$.

2. A creating pair $(K, \Sigma)$ is of the $B C B$-type if it is local, forgetful and satisfies the following condition:

$\left(\circledast \circledast^{\mathrm{BCB}}\right) \quad$ for every sequence $\left\langle s_{n}: n<\omega\right\rangle$ of creatures from $K$ with $m_{\mathrm{dn}}^{s_{n}}=i$, $\operatorname{nor}\left[s_{n}\right] \geq 2$ (for all $n$ ), there are $a_{0}, \ldots, a_{m} \in \mathbf{H}(i)$ and an increasing sequence $\left\langle n_{k}: k<\omega\right\rangle \subseteq \omega$ such that

$\left(\forall a \in \mathbf{H}(i) \backslash\left\{a_{0}, \ldots, a_{m}\right\}\right)\left(\forall^{\infty} k \in \omega\right)\left(\forall u \in \operatorname{dom}\left(\operatorname{val}\left[s_{n_{k}}\right]\right)\right)\left(\langle u, u \smile\langle a\rangle\rangle \in \operatorname{val}\left[s_{n_{k}}\right]\right)$.

3. A local tree-creating pair $(K, \Sigma)$ is of the $\mathrm{BCB}^{\text {tree }}-$ type if

$\left(\circledast_{\text {tree }}^{\mathrm{BCB}}\right) \quad$ for every $\eta \in \bigcup_{n<\omega} \prod_{i<n} \mathbf{H}(i)$ and a sequence $\left\langle s_{n}: n<\omega\right\rangle \subseteq \mathrm{LTCR}_{\eta} \cap K$ such that nor $\left[s_{n}\right] \geq 2$, there are $a_{0}, \ldots, a_{m} \in \mathbf{H}(\operatorname{lh}(\eta))$ and an increasing sequence $\left\langle n_{k}: k<\omega\right\rangle \subseteq \omega$ such that

$$
\left(\forall a \in \mathbf{H}(\operatorname{lh}(\eta)) \backslash\left\{a_{0}, \ldots, a_{m}\right\}\right)\left(\forall^{\infty} k \in \omega\right)\left(\eta \smile\langle a\rangle \in \operatorname{pos}\left(s_{n_{k}}\right)\right) .
$$

Remark 1.4.10. 1. Note that if $\mathbf{H}(i)$ is finite for each $i \in \omega$ then any local forgetful creating pair (local tree creating pair) is of the $\mathrm{BCB}$-type $\left(\mathrm{BCB}^{\text {tree }}\right.$ type, respectively).

2. The difference between $\mathrm{BCB}$ and $\mathrm{BCB}^{\text {tree }}$ is not serious, the two notions are just fitted to their contexts.

3. "BCB" stands for "bounded - co-bounded". The "bounded" part reflects what is stated in (1) above, and the "co-bounded" is supposed to point out the analogy to the co-bounded topology on $\omega$ in the case when each $\mathbf{H}(i)$ is infinite; compare Miller [12].

Theorem 1.4.11. Assume that $h: \omega \times \omega \longrightarrow \omega$ is a regressive function and $\mathcal{F} \subseteq$ $(\omega \backslash 2)^{\omega}$ is a countable $h$-closed family which is $\geq^{*}$-directed.

1. If $(K, \Sigma)$ is a local Cohen-producing h-linked creating pair (tree-creating pair, respectively), then the forcing notion $\mathbb{Q}_{\mathcal{F}}^{*}(K, \Sigma)\left(\mathbb{Q}_{\mathcal{F}}^{\text {tree }}(K, \Sigma)\right.$, resp. $)$ adds a Cohen real. 
2. If $(K, \Sigma)$ is an h-linked tree-creating pair of the $\mathrm{BCB}^{\text {tree }}$-type, then the forcing notion $\mathbb{Q}_{\mathcal{F}}^{\text {tree }}(K, \Sigma)$ is nice.

3. If a creating pair $(K, \Sigma)$ is h-linked and of the $\mathrm{BCB}$-type, then the forcing notion $\mathbb{Q}_{\mathcal{F}}^{*}(K, \Sigma)$ is nice.

Proof. (1) Let $(K, \Sigma)$ be a creating pair for $\mathbf{H}$ and let sets $A_{n} \subseteq \mathbf{H}(n)$ (for $n \in \omega)$ witness that it is Cohen-producing. Let $\dot{c}$ be a $\mathbb{Q}_{\mathcal{F}}^{*}(K, \Sigma)$-name for a real in $2^{\omega}$ defined by

$$
\Vdash_{\mathbb{Q}_{\mathcal{F}}^{*}(K, \Sigma)}(\forall n \in \omega)\left(\dot{c}(n)=1 \Leftrightarrow \dot{W}(n) \in A_{n}\right)
$$

(where $\dot{W}$ is the name for the $\mathbb{Q}_{\mathcal{F}}^{*}(K, \Sigma)$-generic real). Suppose that a condition $p \in \mathbb{Q}_{\mathcal{F}}^{*}(K, \Sigma)$ is such that $\operatorname{nor}\left[t_{n}^{p}\right]>1$ for all $n \in \omega$. Let $\sigma:\left[\operatorname{lh}\left(w^{p}\right), N\right] \longrightarrow 2$, $\operatorname{lh}\left(w^{p}\right) \leq N<\omega$. It should be clear that there is $w \in \operatorname{pos}\left(w^{p}, t_{0}^{p}, \ldots, t_{N-\operatorname{lh}\left(w^{p}\right)}^{p}\right)$ such that

$$
\left(\forall n \in\left[\operatorname{lh}\left(w^{p}\right), N\right]\right)\left(w(n) \in A_{n} \Leftrightarrow \sigma(n)=1\right) .
$$

Hence easily $\dot{c}$ is a name for a Cohen real.

$$
\begin{aligned}
\text { Let } \nu \in \bigcup_{n \in \omega} \prod_{i<n} \mathbf{H}(i), f \in \mathcal{F} \text { and let } \\
P_{\nu, f} \stackrel{\text { def }}{=}\left\{p \in \mathbb{Q}_{\mathcal{F}}^{\text {tree }}(K, \Sigma): \operatorname{root}(p)=\nu \&\left(\forall \eta \in T^{p}\right)\left(\operatorname{nor}\left[t_{\eta}^{p}\right] \geq f(\operatorname{lh}(\eta))\right)\right\} .
\end{aligned}
$$

Suppose that $\left\langle p_{n}: n\langle\omega\rangle \subseteq \mathbb{Q}_{\mathcal{F}}^{\text {tree }}(K, \Sigma)\right.$ is a maximal antichain such that, for each $n \in \omega$ and $\eta \in T^{p_{n}}$, we have $\operatorname{nor}\left[t_{\eta}^{p_{n}}\right] \geq 2$.

Claim 1.4.11.1. There is $N<\omega$ such that

$$
\left(\forall q \in P_{\nu, f}\right)(\exists n<N)\left(q, p_{n} \text { are compatible }\right) .
$$

Proof of the claim. Assume not. Then we may choose a sequence $\left\langle q_{k}: k<\omega\right\rangle \subseteq$ $P_{\nu, f}$ such that for each $n<k<\omega$ the conditions $q_{k}$ and $p_{n}$ are incompatible. We inductively build a tree $T$ and a system $\left\langle s_{\eta}: \eta \in T\right\rangle$ together with a sequence $\left\langle X_{n}, Y_{n}: n<\omega\right\rangle$ so that

$(\alpha) X_{n} \subseteq \bigcup_{m \in \omega} \prod_{i<m} \mathbf{H}(i), Y_{n+1} \subseteq Y_{n} \in[\omega]^{\omega}$,

( $\beta)\left(\forall \eta \in X_{n}\right)\left(\forall^{\infty} k \in Y_{n}\right)\left(\eta \in T^{q_{k}}\right)$,

$(\gamma) \operatorname{nor}\left[s_{\eta}\right] \geq f(\operatorname{lh}(\eta)), T=\bigcup_{n \in \omega} X_{n}$.

Fix a bijection \# : $\bigcup_{m \in \omega} \prod_{i<m} \mathbf{H}(i) \longrightarrow \omega$ such that $\eta_{0} \triangleleft \eta_{1}$ implies \# $\left(\eta_{0}\right)<\#\left(\eta_{1}\right)$.

We declare that $\nu=\operatorname{root}(T), X_{0}=\{\nu\}, Y_{0}=\omega$.

Suppose we have arrived to the $(n+1)^{\text {th }}$ stage of the construction and $X_{n}, Y_{n}$ have been already defined so that the clauses $(\alpha),(\beta)$ above are satisfied. Let $\eta \in X_{n}$ be such that

$$
\#(\eta)=\min \left\{\#\left(\eta^{\prime}\right): \eta^{\prime} \in X_{n}\right\} .
$$

Let $Y_{n}^{\prime} \in[\omega]^{\omega}$ consist of these $k \in Y_{n}$ that $\eta \in T^{q_{k}}($ remember $(\beta))$. Apply $\left(\circledast_{\text {tree }}^{\mathrm{BCB}}\right)$ of 1.4.9 (3) to the sequence $\left\langle t_{\eta}^{q_{k}}: k \in Y_{n}^{\prime}\right\rangle$ to choose an infinite set $Y_{n+1} \subseteq Y_{n}^{\prime}$ such that, letting $k^{*}=\min \left(Y_{n+1}\right)$ and $s_{\eta}=t_{\eta}^{q_{k^{*}}}$ we have

$$
\left(\forall \eta^{\prime} \in \operatorname{pos}\left(s_{\eta}\right)\right)\left(\forall^{\infty} k \in Y_{n+1}\right)\left(\eta^{\prime} \in \operatorname{pos}\left(t_{\eta}^{q_{k}}\right)\right) .
$$

Finally, we let $X_{n+1}=\left(X_{n} \backslash\{\eta\}\right) \cup \operatorname{pos}\left(s_{\eta}\right)$. This finishes the description of the inductive step. 
After the construction is carried out we let $q^{*}=\left\langle s_{\eta}: \eta \in T\right\rangle$. It should be clear that $q^{*} \in \mathbb{Q}_{\mathcal{F}}^{\text {tree }}(K, \Sigma)$ (and even $q^{*} \in P_{\nu, f}$ ). Consequently we find $n<\omega$ such that the conditions $p_{n}$ and $q^{*}$ are compatible.

Suppose that $\nu \unlhd \operatorname{root}\left(p_{n}\right)$. Then necessarily $\operatorname{root}\left(p_{n}\right) \in T$. It follows from our construction (remember clause $(\beta)$ ) that we may find $k>n$ such that $\operatorname{root}\left(p_{n}\right) \in$ $T^{q_{k}}$. But then, using the assumption that $(K, \Sigma)$ is $h$-linked and $\mathcal{F}$ is $h$-closed and $\geq^{*}$-directed, we immediately get that the conditions $p_{n}, q_{k}$ are compatible, contradicting the choice of the $q_{k}$. Similarly, if $\operatorname{root}\left(p_{n}\right) \triangleleft \nu$ then taking any $k>n$ we get that the conditions $q_{k}, p_{n}$ are compatible, again a contradiction.

Since the conditions of the form used above are dense in $\mathbb{Q}_{\mathcal{F}}^{\text {tree }}(K, \Sigma)$ one easily concludes that the forcing notion $\mathbb{Q}_{\mathcal{F}}^{\text {tree }}(K, \Sigma)$ is nice.

Similarly.

1.5. Examples. Our first example recalls the forcing notion of [16, §3]. Let us start with presenting the main tool for this type of constructions - norms determined by Hall's Marriage Theorem.

Definition 1.5.1. Let $\mathbf{H}: \omega \longrightarrow \mathcal{H}\left(\omega_{1}\right)$.

1. Let $\mathcal{K}^{\mathbf{H}}$ be the collection of all finite non-empty families $\Delta$ of finite partial functions $f$ such that $\emptyset \neq \operatorname{dom}(f) \subseteq \omega$ and $f(n) \in \mathbf{H}(n)$ for all $n \in \operatorname{dom}(f)$. For integers $m_{0}<m_{1}$ let

$$
\mathcal{K}_{m_{0}, m_{1}}^{\mathbf{H}} \stackrel{\text { def }}{=}\left\{\Delta \in \mathcal{K}^{\mathbf{H}}:(\forall f \in \Delta)\left(\operatorname{dom}(f) \subseteq\left[m_{0}, m_{1}\right)\right)\right\} .
$$

2. Let $\Delta \in \mathcal{K}^{\mathbf{H}}, k \in \omega$. A function $F: \Delta \longrightarrow[\omega]^{k}$ is a $k$-selector for $\Delta$ if

$$
\left(\forall f, f^{\prime} \in \Delta\right)\left(F(f) \subseteq \operatorname{dom}(f) \text { and } f \neq f^{\prime} \Rightarrow F(f) \cap F\left(f^{\prime}\right)=\emptyset\right) .
$$

3. For $\Delta_{0}, \Delta_{1} \in \mathcal{K}^{\mathbf{H}}$ we write $\Delta_{0} \preceq \Delta_{1}$ whenever

$$
\left(\forall f \in \Delta_{0}\right)\left(\exists g \in \Delta_{1}\right)(g \subseteq f) .
$$

4. We define the Hall norms of a set $\Delta \in \mathcal{K}^{\mathbf{H}}$ as follows:

$\mathbf{h n}^{+}(\Delta) \stackrel{\text { def }}{=} \max \{k+1: k \in \omega$ and there is an $k$-selector for $\Delta\}$,

$\mathbf{h n}(\Delta) \stackrel{\text { def }}{=} \max \left\{k+1: k \in \omega\right.$ and for every $\Delta^{\prime} \subseteq \Delta$ there is $\Delta^{\prime \prime} \subseteq \Delta^{\prime}$ such that elements of $\Delta^{\prime \prime}$ have pairwise disjoint domains and $\left.\left|\bigcup_{f \in \Delta^{\prime \prime}} \operatorname{dom}(f)\right| \geq k \cdot\left|\Delta^{\prime}\right|\right\}$,

$\mathbf{H N}(\Delta) \stackrel{\text { def }}{=} \max \left\{\mathbf{h n}\left(\Delta^{\prime}\right): \Delta \preceq \Delta^{\prime}\right\}$.

Lemma 1.5.2. 1. If $\Delta \in \mathcal{K}^{\mathbf{H}}$ and $k_{0} \in \omega$ then

$$
\begin{aligned}
& \mathbf{h n}^{+}(\Delta)>k_{0} \quad \text { if and only if }\left(\forall \Delta^{\prime} \subseteq \Delta\right)\left(\left|\bigcup\left\{\operatorname{dom}(f): f \in \Delta^{\prime}\right\}\right| \geq k_{0} \cdot\left|\Delta^{\prime}\right|\right) \\
& \text { and } 1 \leq \mathbf{h} \mathbf{n}(\Delta) \leq \mathbf{h n}^{+}(\Delta) \leq \mathbf{H N}(\Delta) \text {. } \\
& \text { 2. If } \Delta_{0}, \Delta_{1} \in \mathcal{K}^{\mathbf{H}} \text { and } \mathbf{x x} \in\left\{\mathbf{h n}, \mathbf{h n}^{+}, \mathbf{H N}\right\} \text { then } \\
& \qquad \mathbf{x x}\left(\Delta_{0} \cup \Delta_{1}\right) \geq\left\lfloor\min \left\{\frac{\mathbf{x x}\left(\Delta_{0}\right)}{2}, \frac{\mathbf{x x}\left(\Delta_{1}\right)}{2}\right\}\right\rfloor .
\end{aligned}
$$


3. If $m_{0}^{0}<m_{1}^{0} \leq m_{0}^{1}<m_{1}^{1} \leq \ldots \leq m_{0}^{k}<m_{1}^{k}<\omega, \Delta_{i} \in \mathcal{K}_{m_{0}^{i}, m_{1}^{i}}^{\mathbf{H}}$ (for $i \leq k$ ) and $\mathbf{x x} \in\left\{\mathbf{h n}, \mathbf{h n}^{+}, \mathbf{H N}\right\}$ then

$$
\mathbf{x x}\left(\bigcup_{i \leq k} \Delta_{i}\right)=\min \left\{\mathbf{x x}\left(\Delta_{i}\right): i \leq k\right\} .
$$

4. Suppose that $m_{0}<m<m_{1}<\omega$ and $\Delta \in \mathcal{K}_{m_{0}, m_{1}}^{\mathbf{H}}$. Let

$$
\begin{aligned}
& \Delta_{0}=\left\{f \uparrow\left[m_{0}, m\right): f \in \Delta \&\left|\operatorname{dom}(f) \cap\left[m_{0}, m\right)\right| \geq \frac{1}{2}|\operatorname{dom}(f)|\right\}, \\
& \Delta_{1}=\left\{f \uparrow\left[m, m_{1}\right): f \in \Delta \&\left|\operatorname{dom}(f) \cap\left[m, m_{1}\right)\right| \geq \frac{1}{2}|\operatorname{dom}(f)|\right\} .
\end{aligned}
$$

Then, for $i<2$, either $\Delta_{i}=\emptyset$ or $\mathbf{h n}\left(\Delta_{i}\right) \geq \frac{1}{2} \mathbf{h n}(\Delta)$.

Proof. (1) It follows from Hall's Theorem (see Hall 画) and the definitions of the norms.

(2)-(4) Straightforward (compare [16, Claim 3.1.2]).

Example 1.5.3. Let $\mathbf{H}: \omega \longrightarrow \mathcal{H}\left(\omega_{1}\right),|\mathbf{H}(n)| \geq 2$ for all $n \in \omega$.

We construct a $\otimes$-creating triple $\left(K_{\mathbf{H}}, \Sigma_{\mathbf{H}}, \Sigma_{\mathbf{H}}^{\perp}\right)$ for $\mathbf{H}$ which:

1. is semi-normal (see 1.4.2(3)), forgetful (see 1.1.2(4)) and super-gluing (see $1.4 .2(4))$,

2. has the cutting property (see 1.1.12(3)),

3. is really finitary (see 1.3.3) provided $\mathbf{H}(n)$ is finite for each $n \in \omega$.

Construction. Let $K_{\mathbf{H}}$ consist of all creatures $t \in \mathrm{CR}[\mathbf{H}]$ such that

- $\operatorname{dis}[t]=\left(m_{\mathrm{dn}}^{t}, m_{\mathrm{up}}^{t}, \Delta_{t}\right)$ for some $\Delta_{t} \in \mathcal{K}_{m_{0}, m_{1}}^{\mathbf{H}} \cup\{\emptyset\}$ such that, if $\Delta_{t} \neq \emptyset$, $\mathbf{h n}^{+}\left(\Delta_{t}\right)>1$,

- $\operatorname{val}[t]=\left\{\langle u, v\rangle \in \prod_{i<m_{\mathrm{dn}}^{t}} \mathbf{H}(i) \times \prod_{i<m_{\mathrm{up}}^{t}} \mathbf{H}(i): u \triangleleft v \&\left(\forall f \in \Delta_{t}\right)(f \nsubseteq v)\right\}$,

- if $\Delta_{t}=\emptyset$ then nor $[t]=m_{\mathrm{dn}}^{t}+1$, otherwise $\operatorname{nor}[t]=\log _{8}\left(\mathbf{h n}\left(\Delta_{t}\right)\right)$.

(Note that $\mathbf{h n}^{+}\left(\Delta_{t}\right)>1$ implies $\operatorname{val}[t] \neq \emptyset$.) For $t_{0}, \ldots, t_{n} \in K_{\mathbf{H}}$ such that $m_{\mathrm{up}}^{t_{i}}=m_{\mathrm{dn}}^{t_{i+1}}($ for $i<n)$ let

$$
\Sigma_{\mathbf{H}}\left(t_{0}, \ldots, t_{n}\right)=\left\{t \in K_{\mathbf{H}}: m_{\mathrm{dn}}^{t}=m_{\mathrm{dn}}^{t_{0}} \& m_{\mathrm{up}}^{t}=m_{\mathrm{up}}^{t_{n}} \& \bigcup_{i \leq n} \Delta_{t_{i}} \subseteq \Delta_{t}\right\} .
$$

It should be clear that $\Sigma_{\mathbf{H}}$ is a composition operation on $K_{\mathbf{H}}, K_{\mathbf{H}}$ is countable and forgetful, and if each $\mathbf{H}(n)$ is finite then $K_{\mathbf{H}}$ is really finitary.

For a creature $t \in K_{\mathbf{H}}$ we define $\Sigma_{\mathbf{H}}^{\perp}(t)$ as follows. It consists of all finite sets $\left\{s_{0}, \ldots, s_{n}\right\} \subseteq K_{\mathbf{H}}$ (a suitable enumeration) such that

- $m_{\mathrm{dn}}^{t}=m_{\mathrm{dn}}^{s_{0}}<m_{\mathrm{up}}^{s_{0}}=m_{\mathrm{dn}}^{s_{1}}<\ldots<m_{\mathrm{up}}^{s_{n-1}}=m_{\mathrm{dn}}^{s_{n}}<m_{\mathrm{up}}^{s_{n}}=m_{\mathrm{up}}^{t}$, and

- $\left(\forall f \in \Delta_{t}\right)(\exists \ell \leq n)\left(f\left\lceil\left[m_{\mathrm{dn}}^{s_{\ell}}, m_{\mathrm{up}}^{s_{\ell}}\right) \in \Delta_{s_{\ell}}\right)\right.$.

It is clear that $\Sigma_{\mathbf{H}}^{\perp}$ is a decomposition operation on $K_{\mathbf{H}}$, so $\left(K_{\mathbf{H}}, \Sigma_{\mathbf{H}}, \Sigma_{\mathbf{H}}^{\perp}\right)$ is a Q-creating triple.

It follows from 1.5.2 $(2)$ that $\left(K_{\mathbf{H}}, \Sigma_{\mathbf{H}}\right)$ is linked, and using 1.5.2(3) one easily shows that it is super-gluing. Similarly, $\left(K_{\mathbf{H}}, \Sigma_{\mathbf{H}}, \Sigma_{\mathbf{H}}^{\perp}\right)$ has the cutting property by $1.5 .2(4)$.

Note that

$(*)$ if $f \in \prod_{i=m_{0}}^{m_{1}-1} \mathbf{H}(i)$, then $\mathbf{h n}(\{f\})=\mathbf{H N}(\{f\})=m_{1}-m_{0}+1$. 
Hence, using 1.5.2 (2), we may easily conclude that $\left(K_{\mathbf{H}}, \Sigma_{\mathbf{H}}\right)$ is reducible. However, it is not normal - one can build $s, t \in K_{\mathbf{H}}$ such that nor $[s]<\operatorname{nor}[t]$ but $\operatorname{val}[t] \subseteq$ $\operatorname{val}[s]$ (which is in some sense paradoxical, and this is why we modify this example in 1.5.5).

Claim 1.5.3.1. $\left(K_{\mathbf{H}}, \Sigma_{\mathbf{H}}, \Sigma_{\mathbf{H}}^{\perp}\right)$ is semi-normal.

Proof of the claim. Let $n \in \omega, t \in K_{\mathbf{H}}$ be such that nor $[t]>2^{n+2}, m_{\mathrm{up}}^{t}-m_{\mathrm{dn}}^{t}>$ $2^{2^{n+4}}$. We may assume that $\Delta_{t} \neq \emptyset$ (remember $(*)$ ). We choose inductively a sequence $\left\langle\Delta_{\ell}, A_{\ell}: \ell \leq n\right\rangle$ such that

1. $\Delta_{\ell} \in \mathcal{K}_{m_{\mathrm{dn}}^{t}, m_{\mathrm{up}}^{t}}^{\mathbf{H}}, A_{\ell} \subseteq \prod_{i=m_{\mathrm{dn}}^{t}}^{m_{\mathrm{up}}^{t}-1} \mathbf{H}(i), A_{\ell} \preceq \Delta_{\ell}$,

2. $\Delta_{0}=\Delta_{t}$,

3. $8^{2^{n+2-\ell}-1} \leq \mathbf{h n}\left(\Delta_{\ell+1}\right)=\mathbf{H N}\left(\Delta_{\ell+1}\right)=\mathbf{H N}\left(A_{\ell+1}\right)<8^{2^{n+2-\ell}}($ for $\ell<n)$,

4. $\left(\forall f \in A_{\ell}\right)\left(\forall g \in \bigcup_{k<\ell} \Delta_{k}\right)(g \nsubseteq f)$.

There are no problems for $\ell=0$ (note that there are practically no restrictions on $A_{0}$, and $\Delta_{0}$ is determined). So suppose that we have arrived to a stage $\ell+1 \leq n$ of the construction. It follows from 1.5.2 $(1,2)$ that $\mathbf{h n}\left(\bigcup_{k \leq \ell} \Delta_{k}\right)>8^{2^{n+3-\ell}-2}$. Let

$$
A^{*}=\left\{f \in \prod_{i=m_{\mathrm{dn}}^{t}}^{m_{\mathrm{up}}^{t}-1} \mathbf{H}(i):\left(\forall g \in \bigcup_{k \leq \ell} \Delta_{k}\right)(g \nsubseteq f)\right\} .
$$

Necessarily $\mathbf{H N}\left(A^{*}\right) \leq 2$. Now, using $(*)$ and 1.5.2 $(2)$, we may choose $A_{\ell+1} \subseteq A^{*}$ such that $8^{2^{n+2-\ell}-1} \leq \mathbf{H N}\left(A_{\ell+1}\right)<8^{2^{n+2-\ell}}$. Next, we pick $\Delta_{\ell+1} \in \mathcal{K}_{m_{\mathrm{dn}}^{t}, m_{\mathrm{up}}^{t}}^{\mathbf{H}}$ satisfying $A_{\ell+1} \preceq \Delta_{\ell+1}$ and $\mathbf{H N}\left(A_{\ell+1}\right)=\mathbf{h n}\left(\Delta_{\ell+1}\right)=\mathbf{H N}\left(\Delta_{\ell+1}\right)$. This finishes the construction.

For $\ell \leq n$ let $s_{\ell} \in \Sigma_{\mathbf{H}}(t)$ be such that $\Delta_{s_{\ell}}=\bigcup_{k \leq \ell} \Delta_{k}$. It should be clear that

$(\alpha) s_{0}=t, s_{\ell+1} \in \Sigma\left(s_{\ell}\right), 2^{n+1-\ell}<\operatorname{nor}\left[s_{\ell+1}\right] \leq 2^{n-\ell+2}($ for $\ell<n)$.

We claim that additionally

$(\beta)$ if $s \in K_{\mathbf{H}}, m_{\mathrm{dn}}^{s}=m_{\mathrm{dn}}^{t}, m_{\mathrm{up}}^{s}=m_{\mathrm{up}}^{t}$ and $\operatorname{nor}[s]>2^{n-\ell+3}, \ell<n$, then

$$
(\forall u \in \operatorname{dom}(\operatorname{val}[s]))(\exists v)\left(\langle u, v\rangle \in \operatorname{val}[s] \backslash \operatorname{val}\left[s_{\ell+1}\right]\right)
$$

(what will finish the proof of the claim). So suppose $s \in K_{\mathbf{H}}, m_{\mathrm{dn}}^{s}=m_{\mathrm{dn}}^{t}, m_{\mathrm{up}}^{s}=$ $m_{\mathrm{up}}^{t}$ and $\operatorname{nor}[s]>2^{n-\ell+3}, \ell<n$. Let $u \in \prod_{i<m_{\mathrm{dn}}^{t}} \mathbf{H}(i)$. If $\Delta_{s}=\emptyset$, then we may take $v \in \prod_{i<m_{\mathrm{up}}^{t}} \mathbf{H}(i)$ such that $u \triangleleft v$ and $\left(\exists f \in \Delta_{t}\right)(f \subseteq v)$, so clearly $\langle u, v\rangle \in \operatorname{val}[s] \backslash \operatorname{val}\left[s_{\ell+1}\right]$. Assume now that $\Delta_{s} \neq \emptyset$, so $\mathbf{h n}\left(\Delta_{s}\right)>8^{2^{n-\ell+3}}$. Since $\mathbf{H N}\left(A_{\ell+1}\right)<8^{2^{n+2-\ell}}<\mathbf{h n}\left(\Delta_{s}\right)$, there is $f \in A_{\ell+1}$ such that $\left(\forall g \in \Delta_{s}\right)(g \nsubseteq f)$. Clearly $\langle u, u \frown f\rangle \in \operatorname{val}[s] \backslash \operatorname{val}\left[s_{\ell+1}\right]$.

Finally note that the forcing notion $\mathbb{Q}_{\infty}^{*}\left(K_{\mathbf{H}}, \Sigma_{\mathbf{H}}, \Sigma_{\mathbf{H}}^{\perp}\right)$ is not trivial.

Conclusion 1.5.4. Let $\mathbf{H}: \omega \longrightarrow \mathcal{H}\left(\omega_{1}\right)$ be such that $|\mathbf{H}(n)| \geq 2$ for all $n<\omega$. Then the forcing notion $\mathbb{Q}_{\infty}^{*}\left(K_{\mathbf{H}}, \Sigma_{\mathbf{H}}, \Sigma_{\mathbf{H}}^{\perp}\right)$ (where $\left(K_{\mathbf{H}}, \Sigma_{\mathbf{H}}, \Sigma_{\mathbf{H}}^{\perp}\right)$ is as defined in 1.5.3) is $\sigma-*-$ linked and it adds a dominating real. Consequently it is not $\omega-$ nw-nep (by [26]). 
If one looks at $\operatorname{val}[t]$ for $t \in K_{\mathbf{H}}$ in 1.5 .3 , then it is clear that $\mathbf{H N}$ is more appropriate to determine the norms of creatures. We presented 1.5.3 as it is a direct relative of the forcing notion of [16, §3]. However, it seems that the following example presents a nicer member of this family.

Example 1.5.5. Let $\mathbf{H}: \omega \longrightarrow \mathcal{H}\left(\omega_{1}\right),|\mathbf{H}(n)| \geq 2$ for all $n \in \omega$.

We construct a $\otimes$-creating triple $\left(K \overline{1.5 .5}, \Sigma_{1.5 .5}, 2\right.$ 店.5.5 $)$ for $\mathbf{H}$ which:

1. is almost normal (see the construction), regular (see 1.3.2), forgetful and super-gluing,

2. has the cutting property,

3. is really finitary provided $\mathbf{H}(n)$ is finite for each $n \in \omega$.

Construction. It is similar to 1.5 .3 , but instead of hn we use HN. So $K \begin{aligned} & 1.5 .5 \\ & \text { consists }\end{aligned}$ of $t \in \mathrm{CR}[\mathbf{H}]$ such that

- $\operatorname{dis}[t]=\left(m_{\mathrm{dn}}^{t}, m_{\mathrm{up}}^{t}, \Delta_{t}\right)$ for some $\Delta_{t} \in \mathcal{K}_{m_{0}, m_{1}}^{\mathbf{H}} \cup\{\emptyset\}$ such that, if $\Delta_{t} \neq \emptyset$, $\mathbf{h n}^{+}\left(\Delta_{t}\right)>1$,

- $\operatorname{val}[t]=\left\{\langle u, v\rangle \in \prod_{i<m_{\mathrm{dn}}^{t}} \mathbf{H}(i) \times \prod_{i<m_{\mathrm{up}}^{t}} \mathbf{H}(i): u \triangleleft v \&\left(\forall f \in \Delta_{t}\right)(f \nsubseteq v)\right\}$,

- if $\Delta_{t}=\emptyset$ then nor $[t]=\log _{8}\left(m_{\mathrm{up}}^{t}-m_{\mathrm{dn}}^{t}\right)+2 m_{\mathrm{dn}}^{t}+1$, otherwise $\operatorname{nor}[t]=$ $\log _{8}\left(\mathbf{H N}\left(\Delta_{t}\right)\right)$.

For $t_{0}, \ldots, t_{n} \in K$ 1.5.5 such that $m_{\mathrm{up}}^{t_{i}}=m_{\mathrm{dn}}^{t_{i+1}}($ for $i<n)$ let

$$
21.5 .5\left(t_{0}, \ldots, t_{n}\right)=\left\{t \in K \text {.1.5.5 }: m_{\mathrm{dn}}^{t}=m_{\mathrm{dn}}^{t_{0}} \& m_{\mathrm{up}}^{t}=m_{\mathrm{up}}^{t_{n}} \& \bigcup_{i \leq n} \Delta_{t_{i}} \subseteq \Delta_{t}\right\} .
$$

For $t \in K$ 1.5.5, 2 1.5.5 $(t)$ consists of all finite sets $\left\{s_{0}, \ldots, s_{n}\right\} \subseteq K$ L.5.5 such that

- $m_{\mathrm{dn}}^{t}=m_{\mathrm{dn}}^{s_{0}}<m_{\mathrm{up}}^{s_{0}}=m_{\mathrm{dn}}^{s_{1}}<\ldots<m_{\mathrm{up}}^{s_{n-1}}=m_{\mathrm{dn}}^{s_{n}}<m_{\mathrm{up}}^{s_{n}}=m_{\mathrm{up}}^{t}$, and

- $\left(\forall f \in \Delta_{t}\right)(\exists \ell \leq n)\left(f\left\lceil\left[m_{\mathrm{dn}}^{s_{\ell}}, m_{\mathrm{up}}^{s_{\ell}}\right) \in \Delta_{s_{\ell}}\right)\right.$.

Clearly $\left(K \frac{1.5 .5}{,}, \Sigma+\frac{1.5 .5}{2}, 2 \frac{1.5 .5}{1}\right)$ is a forgetful super-gluing $\otimes$-creating triple. It is linked, and it is really finitary provided $\mathbf{H}(n)$ is finite for each $n \in \omega$.

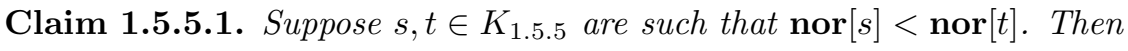

$$
(\forall u \in \operatorname{dom}(\operatorname{val}[t]))(\exists v)(\langle u, v\rangle \in \operatorname{val}[t] \backslash \operatorname{val}[s]) .
$$

Proof of the claim. We may assume that $m_{\mathrm{dn}}^{s}=m_{\mathrm{dn}}^{t}, m_{\mathrm{up}}^{s}=m_{\mathrm{up}}^{t}$ (otherwise trivial). It follows from the assumptions (and the definition of nor $[s]$ ) that $\Delta_{s} \neq \emptyset$. If $\Delta_{t}=\emptyset$, then the conclusion is immediate, so assume $\Delta_{t} \neq \emptyset$. Thus $\Delta_{s}, \Delta_{t} \in \mathcal{K}^{\mathbf{H}}$ and $\mathbf{H N}\left(\Delta_{s}\right)<\mathbf{H N}\left(\Delta_{t}\right)$. Choose $\Delta \in \mathcal{K}^{\mathbf{H}}$ such that $\Delta_{t} \preceq \Delta$ and $\mathbf{h n}(\Delta)=$ $\mathbf{H N}\left(\Delta_{t}\right)$. Note that, by 1.5.2(1), we have $\mathbf{h n}(\Delta)=\mathbf{H N}(\Delta)=\mathbf{h n}^{+}(\Delta)$. Consequently, we may choose $\Delta^{\prime} \in \mathcal{K}^{\mathbf{H}}$ such that elements of $\Delta^{\prime}$ have pairwise disjoint domains, $\Delta \preceq \Delta^{\prime}$ and $\mathbf{h n}\left(\Delta^{\prime}\right)=\mathbf{h n}(\Delta)$. Now, for some $f \in \Delta_{s}$ we have $\left(\forall g \in \Delta^{\prime}\right)(g \nsubseteq f)$. By the choice of $\Delta^{\prime}$ we may build $f^{*} \in \prod_{i=m_{\mathrm{dn}}^{s}}^{m_{\mathrm{up}}^{s}-1} \mathbf{H}(i)$ such that $f \subseteq f^{*}$ and $\left(\forall g \in \Delta^{\prime}\right)\left(g \nsubseteq f^{*}\right)$. Since $\Delta_{t} \preceq \Delta^{\prime}$ we are done.

Using 1.5.5.1 we see that $(K \overline{1.5 .5}, \Sigma$ 1.5.5 $)$ is almost normal in the following sense: the reducibility demand (see 1.4.2(1)) holds for those $t \in K$ 1.5.5 for which $\Delta_{t} \neq \emptyset$. However, this is enough to carry out, e.g., the proof of 1.4.4 (3) with almost no changes.

Claim 1.5.5.2. $(K \overline{1.5 .5}, \Sigma(1.5 .5,2+1.5 .5)$ is regular and has the cutting property. 
Proof of the claim. First we show the regularity. So suppose that

$$
\left(w, t_{0}, \ldots, t_{n}\right),\left(u, s_{0}, \ldots, s_{m}\right) \in \mathrm{FC}(K \text { 1.5.5, } 2 \text { 1.5.5, } 2 \text { 店.5.5 })
$$

are such that $\left(w, t_{0}, \ldots, t_{n}\right) \leq\left(u, s_{0}, \ldots, s_{m}\right), m_{\mathrm{up}}^{s_{m}} \leq m_{\mathrm{up}}^{t_{n}}$, nor $\left[s_{0}\right] \geq 3$, and $\operatorname{nor}\left[t_{\ell}\right] \geq 3$, where $\ell \leq n$ is such that $m_{\mathrm{dn}}^{t_{\ell}}<m_{\mathrm{dn}}^{s_{0}}<m_{\mathrm{up}}^{t_{\ell}} \leq m_{\text {up }}^{s_{0}}$. It follows from the definition of $\leq$ (see 1.1.3) that

$$
\left(\forall f \in \Delta_{t_{\ell}}\right)\left(f\left\lceil m_{\mathrm{dn}}^{s_{0}} \not \subseteq u \text { or }\left(\exists g \in \Delta_{s_{0}}\right)(g \subseteq f)\right) .\right.
$$

Let $t^{\prime}, t^{\prime \prime} \in K$..5.5 be such that $m_{\mathrm{dn}}^{t^{\prime}}=m_{\mathrm{dn}}^{t_{\ell}}, m_{\mathrm{up}}^{t^{\prime}}=m_{\mathrm{dn}}^{t^{\prime \prime}}=m_{\mathrm{dn}}^{s_{0}}, m_{\mathrm{up}}^{t^{\prime \prime}}=m_{\mathrm{up}}^{t_{\ell}}$ and

$$
\begin{aligned}
& \Delta_{t^{\prime}}=\left\{f \left\lceil\left[m_{\mathrm{dn}}^{t_{\ell}}, m_{\mathrm{dn}}^{s_{0}}\right): f \in \Delta_{t_{\ell}} \& f\left\lceil m_{\mathrm{dn}}^{s_{0}} \nsubseteq u\right\},\right.\right. \\
& \Delta_{t^{\prime \prime}}=\left\{f \left\lceil\left(m_{\mathrm{dn}}^{s_{0}}, m_{\mathrm{up}}^{t_{\ell}}\right): f \in \Delta_{t_{\ell}} \& f\left\lceil m_{\mathrm{dn}}^{s_{0}} \subseteq u\right\} .\right.\right.
\end{aligned}
$$

Then $\left\{t^{\prime}, t^{\prime \prime}\right\} \in \Sigma / 1.5 .5\left(t_{\ell}\right)$ and clearly $u \in \operatorname{pos}\left(w, t_{0}, \ldots, t_{\ell-1}, t^{\prime}\right)$. The only thing left is to show that the norm of $t^{\prime \prime}$ is at least 2. If $\Delta_{t^{\prime \prime}}=\emptyset$, then it is clearly true as $m_{\mathrm{dn}}^{t^{\prime \prime}} \geq 1$. So suppose that $\Delta_{t^{\prime \prime}} \neq \emptyset$ and we have to argue that $\mathbf{H N}\left(\Delta_{t^{\prime \prime}}\right) \geq 64$. But this is clear as $\Delta_{t^{\prime \prime}} \preceq \Delta_{s_{0}}$.

Now let us show that $(K(1.5 .5,2,1.5 .5,2(1.5 .5)$ has the cutting property. Let $t \in$ $K{ }_{1.5 .5}, \operatorname{nor}[t]>1, m_{\mathrm{dn}}^{t}<m<m_{\mathrm{up}}^{t}$. Choose $\Delta \in \mathcal{K}_{m_{\mathrm{dn}}^{t}, m_{\mathrm{up}}^{t}}^{\mathbf{H}}$ such that elements of $\Delta$ have pairwise disjoint domains, $\Delta_{t} \preceq \Delta$ and $\mathbf{H N}\left(\Delta_{t}\right)=\mathbf{h n}(\Delta)$ (like in the proof of 1.5.5.1). Put

$$
\begin{aligned}
& \Delta^{0}=\left\{f\left\lceil\left[m_{\mathrm{dn}}^{t}, m\right): f \in \Delta \&\left|\operatorname{dom}(f) \cap\left[m_{\mathrm{dn}}^{t}, m\right)\right| \geq \frac{1}{2}|\operatorname{dom}(f)|\right\},\right. \\
& \Delta^{1}=\left\{f\left\lceil\left[m, m_{\mathrm{up}}^{t}\right): f \in \Delta \&\left|\operatorname{dom}(f) \cap\left[m, m_{\mathrm{up}}^{t}\right)\right| \geq \frac{1}{2}|\operatorname{dom}(f)|\right\} .\right.
\end{aligned}
$$

Let $s_{0}, s_{1} \in K$ L.5.5 be such that $m_{\mathrm{dn}}^{s_{0}}=m_{\mathrm{dn}}^{t}, m_{\mathrm{up}}^{s_{0}}=m=m_{\mathrm{dn}}^{s_{1}}, m_{\mathrm{up}}^{s_{1}}=m_{\mathrm{up}}^{t}$ and

$$
\begin{aligned}
& \Delta_{s_{0}}=\left\{g\left\lceil\left[m_{\mathrm{dn}}^{t}, m\right): g \in \Delta_{t} \&\left(\exists f \in \Delta^{0}\right)(f \subseteq g)\right\},\right. \\
& \Delta_{s_{1}}=\left\{g \uparrow\left[m, m_{\mathrm{up}}^{t}\right): g \in \Delta_{t} \&\left(\exists f \in \Delta^{1}\right)(f \subseteq g)\right\} .
\end{aligned}
$$

Now check.

Conclusion 1.5.6. The forcing notion $\mathbb{Q}_{\infty}^{*}(K \overline{1.5 .5}, \Sigma \sqrt{1.5 .5}, \Sigma(1.5 .5)$ is $\sigma-*-$ linked Souslin ccc and it adds a dominating real. Consequently it adds a Cohen real (by [21]) and it is not $\omega$-nw-nep (by [26]).

Hall's norms are of special interest because of "gluing and cutting", but we may use them to build local creating pairs to.

Example 1.5.7. Let $\mathbf{H}^{*}: \omega \longrightarrow \mathcal{H}\left(\omega_{1}\right), 2 \leq\left|\mathbf{H}^{*}(i)\right|<\omega$ for all $i \in \omega$. Suppose that $\bar{n}=\left\langle n_{k}: k<\omega\right\rangle \subseteq \omega$ is an increasing sequence such that $\lim _{k \rightarrow \infty} n_{k+1}-n_{k}=\infty$ and let $\mathbf{H}=\mathbf{H}^{*}[\bar{n}]: \omega \longrightarrow \mathcal{H}\left(\omega_{1}\right)$ be defined by $\mathbf{H}(k)=\prod_{i=n_{k}}^{n_{k+1}-1} \mathbf{H}^{*}(i)$.

We construct a really finitary creating pair $(K \sqrt{1.5 .7}, 2 \sqrt{1.5 .7})$ for $\mathbf{H}$ which is local, forgetful, normal and Cohen-producing (see 1.4.9(1)).

Construction. Let $K \sqrt{1.5 .7}$ consist of creatures $t \in \mathrm{CR}[\mathbf{H}]$ such that

- $\operatorname{dis}[t]=\left(m_{t}, \Delta_{t}\right)$ for some $m_{t}<\omega$ and $\Delta_{t} \in \mathcal{K}_{n_{m_{t}}, n_{m_{t}+1}}^{\mathbf{H}^{*}} \cup\{\emptyset\}$ such that, if $\Delta_{t} \neq \emptyset, \mathbf{h n}^{+}\left(\Delta_{t}\right)>1$

- $\operatorname{val}[t]=\left\{\langle u, v\rangle \in \prod_{i<m_{t}} \mathbf{H}(i) \times \prod_{i \leq m_{t}} \mathbf{H}(i): u \triangleleft v \&\left(\forall f \in \Delta_{t}\right)\left(f \nsubseteq v\left(m_{t}\right)\right)\right\}$, 
- if $\Delta_{t} \neq \emptyset$, then $\operatorname{nor}[t]=\log _{8}\left(\mathbf{H N}\left(\Delta_{t}\right)\right)$, otherwise $\operatorname{nor}[t]=\log _{8}\left(n_{m_{t}+1}-\right.$ $\left.n_{m_{t}}+1\right)$.

The operation $2 \sqrt{1.5 .7}$ gives non-empty results for singletons only and

$$
2 \text { 1.5.7 }(t)=\left\{s \in K \text { 1.5.7 : } m_{\mathrm{dn}}^{t}=m_{\mathrm{dn}}^{s} \& \Delta_{t} \subseteq \Delta_{s}\right\} .
$$

Clearly, $(K \sqrt{1.5 .7}, \sqrt{1.5 .7})$ is a really finitary creating pair which is local, forgetful and linked (remember 1.5.2(2)).

Claim 1.5.7.1. $(K \sqrt{1.5 .7}, 2 \sqrt{1.5 .7})$ is normal and Cohen-producing.

Proof of the claim. First note that $(K \sqrt{1.5 .7}, \sqrt[2]{1.5 .7})$ is reducible (remember $(*)$ of the construction for 1.5.3; note that here there are no problems caused by $\Delta_{t}=\emptyset$ ). Next note that (the proof of) 1.5.5.1 applies here too.

To show that $\left(K\left[\begin{array}{l}1.5 .7 \\ 1.5 .7\end{array}\right)\right.$ is Cohen-producing fix (for $\left.k \in \omega\right)$ an $a_{k} \in \mathbf{H}^{*}\left(n_{k}\right)$ and let

$$
A_{k}=\left\{f \in \prod_{i=n_{k}}^{n_{k+1}-1} \mathbf{H}^{*}(i): f\left(n_{k}\right)=a_{k}\right\} \subseteq \mathbf{H}(k) .
$$

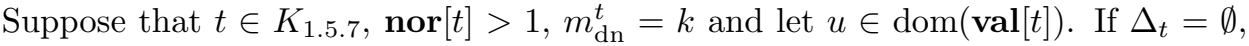
then we easily choose $v_{0}, v_{1} \in \operatorname{rng}(\operatorname{val}[t])$ extending $u$ and such that $v_{0}(k) \notin A_{k}$, $v_{1}(k) \in A_{k}$ (remember $\left|\mathbf{H}^{*}\left(n_{k}\right)\right| \geq 2$ ). So suppose that $\Delta_{t} \neq \emptyset$ and thus $\mathbf{H N}\left(\Delta_{t}\right)>$ 8. Then we may find $\Delta^{\prime} \in \mathcal{K}_{n_{k}, n_{k+1}}^{\mathbf{H}^{*}}$ such that $\Delta_{t} \preceq \Delta^{\prime}$, elements of $\Delta^{\prime}$ have pairwise disjoint domains all of size $>2$. Now we easily build $v_{0}, v_{1} \in \operatorname{dom}(\operatorname{rng}[t])$, both extending $u$ and such that $v_{0}(k) \notin A_{k}, v_{1}(k) \in A_{k}$.

Conclusion 1.5.8. Assume $\mathbf{H}^{*}, \bar{n}, \mathbf{H}$ and $(K \overline{1.5 .7}, \Sigma(1.5 .7)$ are as in 1.5.7.

1. Suppose $f: \omega \times \omega \longrightarrow \omega$ is a fast function such that

$$
(\forall k \in \omega)\left(\forall^{\infty} \ell \in \omega\right)\left(f(k, \ell)<\log _{8}\left(n_{\ell+1}-n_{\ell}\right)\right)
$$

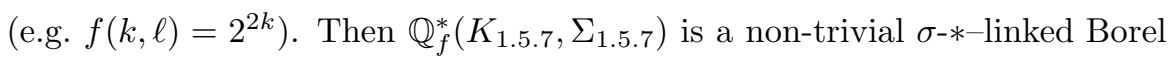
ccc forcing notion which adds a dominating real (so it adds a Cohen real and it is not $\omega-$ nw-nep).

2. Let $h(n, m)=\max \{0, m-1\}$ (so $h: \omega \times \omega \longrightarrow \omega$ is a regressive function). Suppose that $\mathcal{F} \subseteq(\omega \backslash 2)^{\omega}$ is a countable $h$-closed $\geq^{*}$-directed family such that $(\forall f \in \mathcal{F})\left(\forall^{\infty} \ell \in \omega\right)\left(f(\ell)<\log _{8}\left(n_{\ell+1}-n_{\ell}\right)\right)\left(\right.$ e.g. $\mathcal{F}=\left\{f_{k}: k<\omega\right\}$, $\left.f_{k}(\ell)=\max \left\{2,\left\lfloor\frac{1}{k} \log _{8}\left(n_{\ell+1}-n_{\ell}\right)\right\rfloor\right\}\right)$. Then $\mathbb{Q}_{\mathcal{F}}^{*}(K$ 1.5.7, $\Sigma$..5.7 $)$ is a non-trivial $\sigma-*$-linked very Borel ccc forcing notion, it adds a Cohen real and it is nice (so it preserves unbounded families).

Our next examples generalize (in some sense) the Eventually Different Real Forcing of Miller 12 .

Example 1.5.9. Let $\mathbf{H}: \omega \longrightarrow \mathcal{H}\left(\omega_{1}\right),|\mathbf{H}(k)| \geq 4$. For $k<\omega$, let $N_{k}$ be $|\mathbf{H}(k)|$ if $\mathbf{H}(k)$ is finite, and $2^{k+2}$ otherwise. Assume $\lim _{k \mapsto \infty} N_{k}=\infty$. Let $h: \omega \times \omega \longrightarrow \omega$ be given by

$$
h(k, n)= \begin{cases}n-1 & \text { if } n \geq N_{k}, \\ 2 n-N_{k} & \text { if } \frac{7}{8} N_{k}<n<N_{k}, \\ 1 & \text { otherwise. }\end{cases}
$$


(Note that $h$ is regressive.) We construct an $h$-linked creating pair $\left(K\left[\begin{array}{l}1.5 .9 \\ , 2\end{array}\right.\right.$ for $\mathbf{H}$ which is local, forgetful, Cohen-producing and of the BCB-type (see 1.4.9).

Construction. Let $K \overline{1.5 .9}$ be the collection of all $t \in \mathrm{CR}[\mathbf{H}]$ such that

- $\operatorname{dis}[t]=\left(k_{t}, E_{t}\right)$ for some $k_{t}<\omega$ and $E_{t} \subseteq \mathbf{H}\left(k_{t}\right)$ such that $0<\left|E_{t}\right|<N_{k_{t}}$,

- $\operatorname{val}[t]=\left\{\langle u, v\rangle \in \prod_{i<k_{t}} \mathbf{H}(i) \times \prod_{i \leq k_{t}} \mathbf{H}(i): u \triangleleft v \& v\left(k_{t}\right) \notin E_{t}\right\}$,

- if $\left|E_{t}\right| \geq \frac{1}{4} N_{k_{t}}$ then $\operatorname{nor}[t]=1$; otherwise $\operatorname{nor}[t]=N_{k_{t}}-\left|E_{t}\right|$.

The operation $2 \sqrt{1.5 .9}$ is natural: it gives non-empty results for singletons only and

$$
21.5 .9(t)=\left\{s \in K \text { 1.5.9: } k_{s}=k_{t} \& E_{t} \subseteq E_{s}\right\} .
$$

It should be clear that $(K \overline{1.5 .9}, 2 \sqrt{1.5 .9})$ is a local forgetful creating pair for $\mathbf{H}$.

To show that it is $h$-linked suppose that $k>1, t_{0}, t_{1} \in K$ 1.5.9, $\operatorname{nor}\left[t_{0}\right], \operatorname{nor}\left[t_{1}\right] \geq$ $k$ and $\ell=m_{\mathrm{dn}}^{t_{0}}=m_{\mathrm{dn}}^{t_{1}}$. Then $\left|E_{t_{0}}\right|,\left|E_{t_{1}}\right|<\frac{1}{4} N_{\ell}$ and thus $0<\left|E_{t_{0}} \cup E_{t_{1}}\right|<N_{\ell}$. Let $s \in K$ 1.5.9 be such that $k_{s}=\ell, E_{s}=E_{t_{0}} \cup E_{t_{1}}$. Clearly $s \in 2 \sqrt{1.5 .9}\left(t_{0}\right) \cap \Sigma$ 1.5.9 $\left(t_{1}\right)$. If $h(\ell, k)=1$ then clearly nor $[s] \geq h(\ell, k)$, so suppose $h(\ell, k)>1$. Necessarily $\frac{7}{8} N_{\ell}<k$, so $\left|E_{t_{0}}\right|,\left|E_{t_{1}}\right|<\frac{1}{8} N_{\ell}$ and therefore $\left|E_{s}\right|<\frac{1}{4} N_{\ell}$. Hence

$$
\operatorname{nor}[s]=N_{\ell}-\left|E_{s}\right| \geq N_{\ell}-\left|E_{t_{0}}\right|-\left|E_{t_{1}}\right| \geq 2 k-N_{\ell}=h(\ell, k) .
$$

Let us show now that $(K \overline{1.5 .9}, 2 \sqrt{1.5 .9})$ is Cohen-producing. For each $n \in \omega$ choose a set $A_{n} \subseteq \mathbf{H}(n)$ such that $\left|A_{n}\right|=\left[\frac{1}{2}|\mathbf{H}(n)|\right\rfloor$ if $\mathbf{H}(n)$ is finite, and $A_{n}$ is infinite coinfinite if $\mathbf{H}(n)$ is infinite. Suppose that $t \in K\left[1.5 .9\right.$, nor $[t]>1$. Then $E_{t} \subseteq \mathbf{H}\left(k_{t}\right)$, $\left|E_{t}\right|<\frac{1}{4} N_{k_{t}}$, so we may choose $a_{1} \in A_{k_{t}} \backslash E_{k_{t}}$ and $a_{0} \in \mathbf{H}\left(k_{t}\right) \backslash\left(A_{k_{t}} \cup E_{k_{t}}\right)$, and we easily finish.

Finally, let us argue that $(K \overline{1.5 .9}, \Sigma \sqrt{1.5 .9})$ is of the BCB-type, To this end suppose that $\left\langle s_{n}: n<\omega\right\rangle \subseteq K \sqrt{1.5 .9}, m_{\mathrm{dn}}^{s_{n}}=k_{s_{n}}=\ell, \operatorname{nor}\left[s_{n}\right] \geq 2$. Then $\left|E_{s_{n}}\right|<\frac{1}{4} N_{\ell}$ for each $n$.

If $\mathbf{H}(\ell)$ is finite, then the demand in $\left(\circledast^{\mathrm{BCB}}\right)$ of $1.4 .9(2)$ is trivially satisfied (just take $\left.\left\{a_{0}, \ldots, a_{m}\right\}=\mathbf{H}(\ell)\right)$.

So suppose that $\mathbf{H}(\ell)$ is infinite and to simplify notation let $\mathbf{H}(\ell)=\omega$. Let $E_{s_{n}}=$ $\left\{b_{0}^{n}, \ldots, b_{k_{n}-1}^{n}\right\}$ be the increasing enumeration; $k_{n}=\left|E_{s_{n}}\right|$. We may find an infinite set $Y \subseteq \omega$ and $k^{*} \leq k<\omega$ such that

- $k_{n}=k$ for each $n \in Y$,

- $\left\langle b_{i}^{n}: n \in Y\right\rangle$ is constant for each $i<k^{*}$,

- $\left\langle b_{i}^{n}: n \in Y\right\rangle$ is strictly increasing for each $i \in\left[k^{*}, k\right)$.

Suppose $a \in \mathbf{H}(\ell) \backslash\left\{b_{i}^{n}: i<k^{*}\right\}$ for some (equivalently: all) $n \in Y$. Then, for sufficiently large $n \in Y$, for every $i \in\left[k^{*}, k\right)$ we have $b_{i}^{n}>a$. Consequently $\left(\forall^{\infty} n \in Y\right)\left(a \notin E_{s_{n}}\right)$, so we may easily finish.

Conclusion 1.5.10. Let $\mathbf{H},\left\langle N_{k}: k<\omega\right\rangle, h$ and $(K 1.5 .9,21.5 .9)$ be as in 1.5.9. Suppose that $\mathcal{F} \subseteq(\omega \backslash 2)^{\omega}$ is a countable $h$-closed $\geq^{*}$-directed family such that for some $f \in \mathcal{F}$ we have $\left(\forall^{\infty} k \in \omega\right)\left(f(k)<N_{k}\right)$ (e.g. $\mathcal{F}=\left\{f_{\ell}: \ell<\omega\right\}, f_{\ell}(k)=N_{k}-2^{\ell}$ if $N_{k}>2^{\ell+1}, f_{\ell}(k)=2$ otherwise). Then $\mathbb{Q}_{\mathcal{F}}^{*}\left(K{ }_{1.5 .9}, \Sigma(1.5 .9)\right.$ is a non-trivial $\sigma-*-$ linked Borel ccc forcing notion which adds a Cohen real and is nice (so it preserves unbounded families).

If $\mathbf{H}$ and $\mathcal{F}$ are as in 1.5.10, and $\mathbf{H}(k)$ is finite for each $k$, then we may use 1.3.4 (3) to get that the forcing notion $\mathbb{Q}_{\mathcal{F}}^{*}(K \sqrt{1.5 .9}, 5 \sqrt{1.5 .9})$ is very Borel ccc. We may prove the same conclusion without the additional assumption on $\mathbf{H}$ (see 1.5.11 
below). Unfortunately, this proof is very specific for $\mathbb{Q}_{\mathcal{F}}^{*}(K+1.5 .9,2 \sqrt{1.5 .9})$ and it does not generalize to cover more forcing notions of the form $\mathbb{Q}_{\mathcal{F}}^{*}(K, \Sigma)$

Proposition 1.5.11. Assume that $\mathbf{H},\left\langle N_{k}: k<\omega\right\rangle, h, K=K{ }_{1.5 .9}$ and $\Sigma=\sqrt{1.5 .9}$ are as in 1.5.9, and $\mathcal{F} \subseteq(\omega \backslash 2)^{\omega}$ is a countable $h$-closed $\leq^{*}$-directed family such that

$$
(\exists f \in \mathcal{F})\left(\forall^{\infty} k \in \omega\right)\left(f(k)<N_{k}\right) .
$$

Then the forcing notion $\mathbb{Q}_{\mathcal{F}}^{*}(K$..5.9,,$\Sigma$.5.9) is very Borel ccc.

Proof. The only thing that should be shown is that being a maximal antichain is a Borel relation (remember 1.5 .10 and so 1.3.4(1c)). Put $Z=\bigcup_{i<\omega} \prod_{j<i} \mathbf{H}(i) \backslash\{\langle\rangle\}$, $\mathcal{X}=(Z \cup\{\langle\rangle\}) \times K^{\omega}$, and $\mathcal{Y}=\mathcal{X}^{\omega}$ and $\mathcal{Z}=\omega^{Z} \times \omega^{Z}$.

Then $\mathcal{X}, \mathcal{Y}, \mathcal{Z}$ are Polish spaces (each equipped with the respective product topology), $\mathbb{Q}_{\emptyset}^{*}(K, \Sigma)$ and $\mathbb{Q}_{\mathcal{F}}^{*}(K, \Sigma)$ are $\Pi_{1}^{0}$ and $\Sigma_{2}^{0}$ subsets of $\mathcal{X}$, respectively, and for our conclusion it is enough to show that

$$
\begin{aligned}
& P \stackrel{\text { def }}{=}\left\{\left\langle p_{n}: n<\omega\right\rangle \in \mathcal{Y}: \quad(\forall n<\omega)\left(p_{n} \in \mathbb{Q}_{\mathcal{F}}^{*}(K, \Sigma)\right)\right. \text { and } \\
& \left.\left\{p_{n}: n<\omega\right\} \text { is pre-dense in } \mathbb{Q}_{\mathcal{F}}^{*}(K, \Sigma)\right\}
\end{aligned}
$$

is a Borel subset of $\mathcal{Y}$.

Plainly we may assume that

$$
(\forall f \in \mathcal{F})(\forall k<\omega)\left(2 \leq f(k)<N_{k}\right)
$$

(as we may modify suitable the family $\mathcal{F}$ without changing the forcing notion). Now, for $f \in \mathcal{F}$ we define

$$
C_{f}=\left\{\left(h_{0}, h_{1}\right) \in \mathcal{Z}:(\forall \eta \in Z)\left(h_{0}(\eta)<\operatorname{lh}(\eta) \& h_{1}(\eta)<N_{h_{0}(\eta)}-f\left(h_{0}(\eta)\right)\right)\right\} .
$$

Clearly each $C_{f}$ is a compact subset of $\mathcal{Z}$.

Claim 1.5.11.1. Suppose that $\bar{p}=\left\langle p_{n}: n<\omega\right\rangle \in \mathcal{Y}$, where $p_{n} \in \mathbb{Q}_{\mathcal{F}}^{*}(K, \Sigma)$ are such that $\operatorname{nor}\left[t_{k}^{p_{n}}\right]>1$ (for all $k, n<\omega$ ). Then the following are equivalent:

$(\mathrm{A})_{\bar{p}}$ There is a condition $p \in \mathbb{Q}_{\mathcal{F}}^{*}(K, \Sigma)$ incompatible with every $p_{n}$ (for $\left.n<\omega\right)$.

$(\mathrm{B})_{\bar{p}}$ There are eta $\in Z$ and $f \in \mathcal{F}$ and $\left(h_{0}, h_{1}\right) \in C_{f}$ such that

(i) for every $\nu \in Z$, if $\eta \triangleleft \nu$ then $h_{0}(\nu) \geq \operatorname{lh}(\eta)$, and

(ii) for every $\nu_{0}, \nu_{1} \in Z$, if $\eta \triangleleft \nu_{0}, \eta \triangleleft \nu_{1}$ and $\nu_{0}, \nu_{1} \in \bigcup_{n<\omega} \operatorname{POS}\left(p_{n}\right)$ and $h_{0}\left(\nu_{0}\right)=h_{0}\left(\nu_{1}\right)$ and $h_{1}\left(\nu_{0}\right)=h_{1}\left(\nu_{1}\right)$, then $\nu_{0}\left(h_{0}\left(\nu_{0}\right)\right)=\nu_{1}\left(h_{0}\left(\nu_{1}\right)\right)$.

Proof of the claim. Assume $(\mathrm{A})_{\bar{p}}$ and pick $p \in \mathbb{Q}_{\mathcal{F}}^{*}(K, \Sigma)$ and $f \in \mathcal{F}$ such that

$$
(\forall k<\omega)\left(\operatorname{nor}\left[t_{k}^{p}\right]=N_{\operatorname{lh}\left(w^{p}\right)+k}-\left|E_{t_{k}^{p}}\right|=f\left(\operatorname{lh}\left(w^{p}\right)+k\right)\right),
$$

and $p$ is incompatible with all $p_{n}$ (for $n<\omega$ ). For $\ell=\operatorname{lh}\left(w^{p}\right)+k, k<\omega$, let $\left\langle x_{m}^{\ell}: m<N_{\ell}-f(\ell)\right\rangle$ be an enumeration of $E_{t_{k}^{p}}$. Note that, as $p \perp p_{n}$, if $\nu \in \operatorname{POS}\left(p_{n}\right)$ and $w^{p} \triangleleft \nu$, then for some $h_{0}(\nu) \in\left[\operatorname{lh}\left(w^{p}\right), \operatorname{lh}(\nu)\right)$ and $h_{1}(\nu)<N_{h_{0}(\nu)}-f\left(h_{0}(\nu)\right)$ we have $\nu\left(h_{0}(\nu)\right)=x_{h_{1}(\nu)}^{h_{0}(\nu)}$. Letting $\eta=w^{p}$ we may now easily define $\left(h_{0}, h_{1}\right)$ so that $\eta, f,\left(h_{0}, h_{1}\right)$ witness $(\mathrm{B})_{\bar{p}}$.

Suppose now that $\eta, f$ and $\left(h_{0}, h_{1}\right)$ witness $(\mathrm{B})_{\bar{p}}$. Let $w^{p}=\eta$ and for $\ell=$ $\operatorname{lh}\left(w^{p}\right)+k, k<\omega$ and $m<N_{\ell}-f(\ell)$ let $x_{m}^{\ell} \in \mathbf{H}(\ell)$ be such that

$(*)$ if $\nu \in \bigcup \operatorname{POS}\left(p_{n}\right), w^{p} \triangleleft \nu$ and $h_{0}(\nu)=\ell$ and $h_{1}(\nu)=m$, then $x_{m}^{\ell}=\nu(\ell)$. 
(The choice of the $x_{m}^{\ell}$ 's is possible by (B) $)_{\bar{p}}(\mathrm{ii})$. ) Now, for each $k<\omega$ pick $t_{k}^{p} \in K$ so that

$$
m_{\mathrm{dn}}^{t_{k}^{p}}=\operatorname{lh}\left(w^{p}\right)+k \quad \text { and } \quad E_{t_{k}^{p}}=\left\{x_{m}^{\operatorname{lh}\left(w^{p}\right)+k}: m<N_{\operatorname{lh}\left(w^{p}\right)+k}-f\left(\operatorname{lh}\left(w^{p}\right)+k\right)\right\} .
$$

Notice that for sufficiently large $k$ we have $f\left(\operatorname{lh}\left(w^{p}\right)+k\right)>\frac{7}{8} N_{\operatorname{lh}\left(w^{p}\right)+k}$, so for those $k$ we will also have nor $\left[t_{k}^{p}\right]=f\left(\operatorname{lh}\left(w^{p}\right)+k\right)$. Hence $p \in \mathbb{Q}_{\mathcal{F}}^{*}(K, \Sigma)$ and easily it is a condition incompatible with all $p_{n}$ 's.

For $\eta \in Z$ and $f \in \mathcal{F}$, let $B^{\eta, f}$ consist of all $\left(h_{0}, h_{1}, \bar{p}\right) \in \mathcal{Z} \times \mathcal{Y}$ such that

- $\left(h_{0}, h_{1}\right) \in C_{f}, \bar{p}=\left\langle p_{n}: n<\omega\right\rangle \in \mathcal{Y}$, where $p_{n} \in \mathbb{Q}_{\emptyset}^{*}(K, \Sigma)$ are such that $\operatorname{nor}\left[t_{k}^{p_{n}}\right]>1$ for all $k, n<\omega$, and

- $(\forall \nu \in Z)\left(\eta \triangleleft \nu \Rightarrow \operatorname{lh}(\eta) \leq h_{0}(\nu)\right)$, and

- for all $\nu_{0}, \nu_{1} \in Z \cap \bigcup_{n<\omega} \operatorname{POS}\left(p_{n}\right)$ we have

$$
h_{0}\left(\nu_{0}\right)=h_{0}\left(\nu_{1}\right) \& h_{1}\left(\nu_{0}\right)=h_{1}\left(\nu_{1}\right) \quad \Rightarrow \quad \nu_{0}\left(h_{0}\left(\nu_{0}\right)\right)=\nu_{1}\left(h_{1}\left(\nu_{1}\right)\right) .
$$

It should be clear that $B^{\eta, f}$ is a closed subset of $C_{f} \times \mathcal{Y}$ and hence (as $C_{f}$ is compact) the set

$$
A^{\eta, f}=\left\{\bar{p} \in \mathcal{Y}:\left(\exists\left(h_{0}, h_{1}\right) \in C_{f}\right)\left(\left(h_{0}, h_{1}, \bar{p}\right) \in B^{\eta, f}\right)\right\}
$$

is a closed subset of $\mathcal{Y}$, and $A^{\eta, f} \cap\left(\mathbb{Q}_{\mathcal{F}}^{*}(K, \Sigma)\right)^{\omega}$ is Borel. Now, pick a Borel function $\pi: \mathcal{Y} \longrightarrow \mathcal{Y}$ such that

if $\bar{p}=\left\langle p_{n}: n<\omega\right\rangle \in\left(\mathbb{Q}_{\mathcal{F}}^{*}(K, \Sigma)\right)^{\omega}$ and $\bar{q}=\left\langle q_{n}: n<\omega\right\rangle=\pi(\bar{p})$,

then

- for each $n<\omega, q_{n} \in \mathbb{Q}_{\mathcal{F}}^{*}(K, \Sigma)$ and $(\forall k<\omega)\left(\operatorname{nor}\left[t_{k}^{q_{n}}\right]>1\right)$,

- for each $n<\omega$, for some $m<\omega$ and $k<\omega$ we have

$$
w^{q_{n}} \in \operatorname{pos}\left(w^{p_{m}}, t_{0}^{p_{m}}, \ldots, t_{k}^{p_{m}}\right) \quad \text { and } \quad q_{n}=\left(w^{q_{n}}, t_{k+1}^{p_{m}}, t_{k+2}^{p_{m}}, \ldots\right),
$$

- if $m<\omega, k<\omega, w \in \operatorname{pos}\left(w^{p_{m}}, t_{0}^{p_{m}}, \ldots, t_{k}^{p_{m}}\right)$, and $(\forall \ell>k)\left(\operatorname{nor}\left[t_{\ell}^{p_{m}}\right]>1\right)$, then $\left(w, t_{k+1}^{p_{m}}, t_{k+2}^{p_{m}}, \ldots\right) \in\left\{q_{n}: n<\omega\right\}$.

Clearly, $\bar{p} \in\left(\mathbb{Q}_{\mathcal{F}}^{*}(K, \Sigma)\right)^{\omega}$ is pre-dense if and only if so is $\pi(\bar{p})$. Hence, for $\bar{p} \in$ $\left(\mathbb{Q}_{\mathcal{F}}^{*}(K, \Sigma)\right)^{\omega}$ we have

$\bar{p}$ is pre-dense if and only if

there are $\eta \in Z$ and $f \in \mathcal{F}$ such that $\pi(\bar{p}) \in A^{\eta, f}$

(remember 1.5.11.1). Since both $Z$ and $\mathcal{F}$ are countable, the proof of the proposition is completed.

The construction presented in 1.5.9 is a particular case of a more general method of building linked creating pairs from some of the examples presented in 15 . First let us recall the following definition.

Definition 1.5.12 (See [15, Def. 5.2.5]). Let $(K, \Sigma)$ be a creating pair. We say that a creature $t \in K$ is $(n, m)$-additive if for all $t_{0}, \ldots, t_{n-1} \in \Sigma(t)$ such that $\operatorname{nor}\left[t_{i}\right] \leq m$ (for $\left.i<n\right)$ there is $s \in \Sigma(t)$ such that

$$
t_{0}, \ldots, t_{n-1} \in \Sigma(s) \quad \text { and } \quad \operatorname{nor}[s] \leq \max \left\{\operatorname{nor}\left[t_{\ell}\right]: \ell<n\right\}+1 \text {. }
$$

Example 1.5.13. Suppose that $(K, \Sigma)$ is a local and forgetful creating pair for $\mathbf{H}$, and it satisfies the demand $(\boxplus)$ of $1.4 .2(2)$. Let $\bar{t}^{*}=\left\langle t_{0}^{*}, t_{1}^{*}, t_{2}^{*}, \ldots\right\rangle \in \mathrm{PC}_{\infty}(K, \Sigma)$ be such that each $t_{n}^{*}$ is $\left(2, \operatorname{nor}\left[t_{n}^{*}\right]\right)$-additive and $m_{\mathrm{dn}}^{t_{0}}=0$. We construct a local linked creating pair $\left(K_{\bar{t}^{*}}^{c}, \Sigma_{\bar{t}^{*}}^{c}\right)\left(\right.$ the $\bar{t}^{*}$-dual of $\left.(K, \Sigma)\right)$. 
Construction. For $n<\omega$ and a creature $t \in \Sigma\left(t_{n}^{*}\right)$ let a creature $t^{c}$ be such that

- $\operatorname{nor}\left[t^{c}\right]=\max \left\{0, \operatorname{nor}\left[t_{n}^{*}\right]-\operatorname{nor}[t]\right\}$,

- $\operatorname{val}\left[t^{c}\right]=\left(\prod_{i<n} \mathbf{H}(i) \times \prod_{i \leq n} \mathbf{H}(i)\right) \backslash \mathbf{v a l}[t]$

- $\operatorname{dis}\left[t^{c}\right]=(\operatorname{dis}[t], c)$.

[The creature $t^{c}$ is defined only if $\operatorname{val}\left[t^{c}\right] \neq \emptyset$.] Let $K_{\bar{t}^{*}}^{c}$ be the collection of all (correctly defined) $t^{c}$ (for $t \in \Sigma\left(t_{n}^{*}\right), n<\omega$ ). For $t^{c} \in K_{\bar{t}^{*}}^{c}$ (defined as above for $\left.t \in \Sigma\left(t_{n}^{*}\right)\right)$ we let

$$
\Sigma_{t^{*}}^{c}\left(t^{c}\right)=\left\{s^{c}: t \in \Sigma(s) \& s \in \Sigma\left(t_{n}^{*}\right)\right\} .
$$

The examples of local creating pairs have their (local) tree-creating variants too. They can be constructed like the following example.

Example 1.5.14. Let $\mathbf{H} \in \omega^{\omega}$ be a strictly increasing function such that $\mathbf{H}(0)>$ 2 . We construct a really finitary, normal (local) tree-creating pair $(K 1.5 .14,21.5 .14)$ for $\mathbf{H}$ which is Cohen-producing.

Construction. The family $K$. 5.14 consists of tree-creatures $t \in \operatorname{LTCR}[\mathbf{H}]$ such that

- $\operatorname{dis}[t]=\left(m_{t}, \eta_{t}, E_{t}\right)$ such that $m_{t}<\omega, \eta_{t} \in \prod_{i<m_{t}} \mathbf{H}(i)$ and $\emptyset \neq E_{t} \subseteq \mathbf{H}\left(m_{t}\right)$, $E_{t} \neq \mathbf{H}\left(m_{t}\right)$,

- $\operatorname{val}[t]=\left\{\left\langle\eta_{t}, \nu\right\rangle: \eta_{t} \triangleleft \nu \in \prod_{i \leq m_{t}} \mathbf{H}(i) \& \nu\left(m_{t}\right) \notin E_{t}\right\}$,

- $\operatorname{nor}[t]=\log _{4}\left(\frac{\mathbf{H}\left(m_{t}\right)}{\left|E_{t}\right|}\right)$.

The tree composition $\Sigma 1.5 .14$ is natural: it gives non empty results for singletons only and then

$$
21.5 .14(t)=\left\{s \in K \text { 1.5.14 }: \eta_{s}=\eta_{t} \& E_{t} \subseteq E_{s}\right\} .
$$

Now check.

Conclusion 1.5.15. Suppose $\mathbf{H} \in \omega^{\omega}$ is strictly increasing, $\mathbf{H}(0)>4$.

1. The forcing notion $\mathbb{Q}_{1}^{\text {tree }}(K \overline{1.5 .14}, 2 \longdiv { 1 . 5 . 1 4 })$ is non-trivial, $\sigma-*$-linked and it adds a dominating real.

2. Assume that $f: \omega \times \omega \longrightarrow \omega$ is a fast function such that

$$
(\forall n, m<\omega)\left(f(n, m)<\log _{4}(\mathbf{H}(m))\right) .
$$

Then the forcing notion $\mathbb{Q}_{f}^{\text {tree }}(K \overline{1.5 .14}, 2 \sqrt{1.5 .14})$ is non-trivial, $\sigma-*-$ linked, Borel ccc, and it adds a dominating real.

3. Suppose that $\mathcal{F} \subseteq(\omega \backslash 2)^{\omega}$ is a countable $\geq^{*}$-directed family such that

$$
\begin{aligned}
& (\exists f \in \mathcal{F})\left(\forall^{\infty} n \in \omega\right)\left(f(n)<\log _{4}(\mathbf{H}(n))\right) \quad \text { and } \\
& (\forall f \in \mathcal{F})(\exists g \in \mathcal{F})\left(\forall^{\infty} n \in \omega\right)(g(n)<f(n)-1) .
\end{aligned}
$$

Then the forcing notion $\mathbb{Q}_{\mathcal{F}}^{\text {tree }}(K \overline{1.5 .14}, 2 \sqrt{1.5 .14})$ is non-trivial, $\sigma-*-$ linked, very Borel ccc, and it adds a Cohen real and is nice.

\section{More constructions}

In this section we introduce more schemes for building ccc forcing notions as well as more norm conditions that can be used in conjunctions with the methods presented in the previous section. 


\subsection{Mixtures with random.}

Definition 2.1.1. Let $\mathbf{H}: \omega \longrightarrow \mathcal{H}\left(\omega_{1}\right)$. We say that $(K, \Sigma, \mathbf{F})$ is a mixing triple for $\mathbf{H}$ if

(a) $(K, \Sigma)$ is a (local) tree-creating pair for $\mathbf{H}$,

(b) for each $\eta \in \bigcup_{m \in \omega} \prod_{i<m} \mathbf{H}(i)$ there is $t_{\eta}^{*} \in K$ such that

$$
\left(\forall t \in \operatorname{LTCR}_{\eta}[\mathbf{H}] \cap K\right)\left(t \in \Sigma\left(t_{\eta}^{*}\right)\right),
$$

(c) $\mathbf{F}=\left\langle F_{\eta}: \eta \in \bigcup_{m \in \omega} \prod_{i<m} \mathbf{H}(i)\right\rangle$, where for each $\eta \in \bigcup_{m \in \omega} \prod_{i<m} \mathbf{H}(i)$ :

(d) $F_{\eta}:[0,1]^{\operatorname{pos}\left(t_{\eta}^{*}\right)} \longrightarrow[0,1]$,

(e) if $\left\langle r_{\nu}: \nu \in \operatorname{pos}\left(t_{\eta}^{*}\right)\right\rangle,\left\langle r_{\nu}^{\prime}: \nu \in \operatorname{pos}\left(t_{\eta}^{*}\right)\right\rangle \in[0,1]^{\operatorname{pos}\left(t_{\eta}^{*}\right)}, r_{\nu} \leq r_{\nu}^{\prime}$ for all $\nu \in$ $\operatorname{pos}\left(t_{\eta}^{*}\right)$, then $F_{\eta}\left(r_{\nu}: \nu \in \operatorname{pos}\left(t_{\eta}^{*}\right)\right) \leq F_{\eta}\left(r_{\nu}^{\prime}: \nu \in \operatorname{pos}\left(t_{\eta}^{*}\right)\right)$,

(f) if $\left\langle r_{\nu}: \nu \in \operatorname{pos}\left(t_{\eta}^{*}\right)\right\rangle \in[0,1]^{\operatorname{pos}\left(t_{\eta}^{*}\right)}, \varepsilon>0$, then there are $r_{\nu}^{\prime}<r_{\nu}$ (for $\left.\nu \in \operatorname{pos}\left(t_{\eta}^{*}\right)\right)$ such that for each $\left\langle r_{\nu}^{\prime \prime}: \nu \in \operatorname{pos}\left(t_{\eta}^{*}\right)\right\rangle \in[0,1]^{\operatorname{pos}\left(t_{\eta}^{*}\right)}$ satisfying $r_{\nu}^{\prime}<r_{\nu}^{\prime \prime} \leq r_{\nu}\left(\right.$ for $\left.\nu \in \operatorname{pos}\left(t_{\eta}^{*}\right)\right)$ we have

$$
F_{\eta}\left(r_{\nu}: \nu \in \operatorname{pos}\left(t_{\eta}^{*}\right)\right)-\varepsilon<F_{\eta}\left(r_{\nu}^{\prime \prime}: \nu \in \operatorname{pos}\left(t_{\eta}^{*}\right)\right),
$$

(g) if $r_{\nu} \geq \varepsilon>0$ for $\nu \in \operatorname{pos}\left(t_{\eta}^{*}\right)$ then $F_{\eta}\left(r_{\nu}: \nu \in \operatorname{pos}\left(t_{\eta}^{*}\right)\right) \geq \varepsilon$.

Definition 2.1.2. Let $(K, \Sigma, \mathbf{F})$ be a mixing triple for $\mathbf{H}$.

1. Let $T^{*}=T_{K, \Sigma}^{*} \subseteq \bigcup_{m \in \omega} \prod_{i<m} \mathbf{H}(i)$ be a tree such that

$$
\operatorname{root}\left(T^{*}\right)=\langle\rangle \quad \text { and } \quad\left(\forall \eta \in T^{*}\right)\left(\operatorname{succ}_{T^{*}}(\eta)=\operatorname{pos}\left(t_{\eta}^{*}\right)\right) .
$$

2. If $X \subseteq \operatorname{pos}\left(t_{\eta}^{*}\right), \eta \in T^{*}$ and $\left\langle r_{\nu}: \nu \in X\right\rangle \subseteq[0,1]$, then we define $F_{\eta}\left(r_{\nu}: \nu \in\right.$ $X)$ as $F_{\eta}\left(r_{\nu}^{*}: \nu \in \operatorname{pos}\left(t_{\eta}^{*}\right)\right)$, where

$$
r_{\nu}^{*}= \begin{cases}r_{\nu} & \text { if } \nu \in X \\ 0 & \text { if } \nu \in \operatorname{pos}\left(t_{\eta}^{*}\right) \backslash X .\end{cases}
$$

3. Suppose that $p=\left\langle t_{\eta}^{p}: \eta \in T^{p}\right\rangle \in \mathbb{Q}_{\emptyset}^{\text {tree }}(K, \Sigma)$ and $A \subseteq T^{p}$ is a front of $T^{p}$. We let $T[p, A]=\left\{\eta \in T^{p}:(\exists \rho \in A)(\eta \unlhd \rho)\right\}$, and we define $\mu_{p, A}^{\mathbf{F}}=\mu_{p, A}$ : $T[p, A] \longrightarrow[0,1]$ by downward induction as follows:

- if $\eta \in A$ then $\mu_{p, A}(\eta)=1$,

- if $\mu_{p, A}(\nu)$ has been defined for all $\nu \in \operatorname{pos}\left(t_{\eta}^{p}\right), \eta \in T[p, A] \backslash A$, then we put $\mu_{p, A}(\eta)=F_{\eta}\left(\mu_{p, A}(\nu): \nu \in \operatorname{pos}\left(t_{\eta}^{p}\right)\right)$.

4. For $p=\left\langle t_{\eta}^{p}: \eta \in T^{p}\right\rangle \in \mathbb{Q}_{\emptyset}^{\text {tree }}(K, \Sigma)$ we define

$$
\mu^{\mathbf{F}}(p)=\inf \left\{\mu_{p, A}(\operatorname{root}(p)): A \text { is a front of } T^{p}\right\} .
$$

5. Let $\mathbb{Q}_{\emptyset}^{\mathrm{mt}}(K, \Sigma, \mathbf{F})=\left\{p \in \mathbb{Q}_{\emptyset}^{\text {tree }}(K, \Sigma): \mu^{\mathbf{F}}(p)>0\right\}$ be equipped with the partial order inherited from $\mathbb{Q}_{\emptyset}^{\text {tree }}(K, \Sigma)$. Similarly we define forcing notions $\mathbb{Q}_{1}^{\mathrm{mt}}(K, \Sigma, \mathbf{F}), \mathbb{Q}_{f}^{\mathrm{mt}}(K, \Sigma, \mathbf{F}), \mathbb{Q}_{\mathcal{F}}^{\mathrm{mt}}(K, \Sigma, \mathbf{F})$ (for suitable $f$ and $\left.\mathcal{F}\right)$.

Definition 2.1.3. A mixing triple $(K, \Sigma, \mathbf{F})$ is $c c c-$ complete if

(a) for each $\eta \in T_{K, \Sigma}^{*}$ and $A \subseteq \operatorname{pos}\left(t_{\eta}^{*}\right)$, there is a unique tree-creature $t_{A} \in \Sigma\left(t_{\eta}^{*}\right)$ such that $\operatorname{pos}\left(t_{A}\right)=A$,

(b) if $\eta \in T_{K, \Sigma}^{*}, A \subseteq B \subseteq \operatorname{pos}\left(t_{\eta}^{*}\right)$, then $t_{A} \in \Sigma\left(t_{B}\right)$ and $\operatorname{nor}\left[t_{A}\right] \leq \operatorname{nor}\left[t_{B}\right]$, 
(c) if $r_{\nu}=r_{\nu}^{\prime}+r_{\nu}^{\prime \prime}, r_{\nu}, r_{\nu}^{\prime}, r_{\nu}^{\prime \prime} \in[0,1]$ (for $\left.\nu \in \operatorname{pos}\left(t_{\eta}^{*}\right), \eta \in T_{K, \Sigma}^{*}\right)$, then

$$
F_{\eta}\left(r_{\nu}: \nu \in \operatorname{pos}\left(t_{\eta}^{*}\right)\right)=F_{\eta}\left(r_{\nu}^{\prime}: \nu \in \operatorname{pos}\left(t_{\eta}^{*}\right)\right)+F_{\eta}\left(r_{\nu}^{\prime \prime}: \nu \in \operatorname{pos}\left(t_{\eta}^{*}\right)\right) \text {. }
$$

Lemma 2.1.4. Let $(K, \Sigma, \mathbf{F})$ be a ccc-complete mixing triple for $\mathbf{H}$. Suppose that $p_{0}, \ldots p_{m} \in \mathbb{Q}_{\emptyset}^{\mathrm{mt}}(K, \Sigma, \mathbf{F})$ are such that $\sum_{\ell \leq m} \mu^{\mathbf{F}}\left(p_{\ell}\right)>1$ and $\operatorname{root}\left(p_{0}\right)=\ldots=$ $\operatorname{root}\left(p_{m}\right)$. Then for some $\ell<n \leq m$ the conditions $p_{\ell}, p_{n}$ are compatible in $\mathbb{Q}_{\emptyset}^{\mathrm{mt}}(K, \Sigma, \mathbf{F})$.

Proof. Let $\nu=\operatorname{root}\left(p_{0}\right)=\ldots=\operatorname{root}\left(p_{m}\right)$. For each $\ell<n \leq m$ such that $\left[T^{p_{\ell}}\right] \cap$ $\left[T^{p_{n}}\right] \neq \emptyset$ choose a tree $T_{\ell, n} \subseteq T_{K, \Sigma}^{*}$ satisfying

$$
\max \left(T_{\ell, n}\right)=\emptyset, \quad \operatorname{root}\left(T_{\ell, n}\right)=\nu \quad \text { and } \quad\left[T_{\ell, n}\right]=\left[T^{p_{\ell}}\right] \cap\left[T^{p_{n}}\right] .
$$

Let $p_{\ell, n} \in \mathbb{Q}_{\emptyset}^{\text {tree }}(K, \Sigma)$ be such that $T^{p_{\ell, n}}=T_{\ell, n}$ (defined if $\left[T^{p_{\ell}}\right] \cap\left[T^{p_{n}}\right] \neq \emptyset$, $\ell<n \leq m)$. Our aim is to show that for some $\ell<n \leq m, p_{\ell, n}$ is defined and belongs to $\mathbb{Q}_{\emptyset}^{\mathrm{mt}}(K, \Sigma, \mathbf{F})$ (i.e., $\mu^{\mathbf{F}}\left(p_{\ell, n}\right)>0$ ). So suppose that for each $\ell<n \leq m$, either $\left[T^{p_{\ell}}\right] \cap\left[T^{p_{n}}\right]=\emptyset$ or $\mu^{\mathbf{F}}\left(p_{\ell, n}\right)=0$. Let $\varepsilon=2^{-(m+1)}\left(\sum_{\ell \leq m} \mu^{\mathbf{F}}\left(p_{\ell}\right)-1\right)>0$ and let $p \in \mathbb{Q}_{\emptyset}^{\text {tree }}(K, \Sigma)$ be such that $\operatorname{root}(p)=\nu$ and $T^{p}=T^{p_{0}} \cup \ldots \cup T^{p_{m}}$ (clearly $\left.\min \left\{\mu^{\mathbf{F}}\left(p_{\ell}\right): \ell \leq m\right\} \leq \mu^{\mathbf{F}}(p) \leq 1\right)$. Choose a front $A$ of $T^{p}$ such that for each $\ell<n \leq m$, if $p_{\ell, n}$ is defined and $A_{\ell, n}=A \cap T^{p_{\ell, n}}$, then $\mu_{p_{\ell, n}, A_{\ell, n}}(\nu)<\varepsilon$, and if $\left[T^{p_{\ell}}\right] \cap\left[T^{p_{n}}\right]=\emptyset$ then $\left.T^{p_{\ell}} \cap T^{p_{n}} \subseteq T[p, A] \backslash A\right)$.

Claim 2.1.4.1. For each $\eta \in T[p, A]$ we have

$$
\mu_{p, A}(\eta) \geq \sum_{\ell \leq m} \mu_{p_{\ell}, A^{\ell}}(\eta)-\sum_{\ell<n \leq m} \mu_{p_{\ell, n}, A_{\ell, n}}(\eta),
$$

where $A^{\ell}=A \cap T^{p_{\ell}}$ (for $\ell \leq m$ ), and if $\ell<n \leq m$ and $p_{\ell, n}$ is not defined or $\eta \notin T\left[p_{\ell, n}, A_{\ell, n}\right]$ then we stipulate $\mu_{p_{\ell, n}, A_{\ell, n}}(\eta)=0$ (and similarly $\mu_{p_{\ell}, A^{\ell}}(\eta)=0$ if $\left.\eta \notin T\left[p_{\ell}, A^{\ell}\right]\right)$.

Proof of the claim. We show this by downward induction on $\operatorname{lh}(\eta)$.

First suppose that $\eta \in A$. Let $k=\left|\left\{\ell \leq m: \eta \in A^{\ell}\right\}\right|=\sum_{\ell \leq m} \mu_{p_{\ell}, A^{\ell}}(\eta)$. Then $\sum_{\ell<n \leq m} \mu_{p_{\ell, n}, A_{\ell, n}}(\eta)=\left(\begin{array}{c}k \\ 2\end{array}\right)$ and

$$
\sum_{\ell \leq m} \mu_{p_{\ell}, A^{\ell}}(\eta)-\sum_{\ell<n \leq m} \mu_{p_{\ell, n}, A_{\ell, n}}(\eta)=k-\frac{k(k-1)}{2} \leq 1=\mu_{p, A}(\eta) .
$$

Suppose now that $(\otimes)$ has been shown for all $\rho \in \operatorname{pos}\left(t_{\eta}^{p}\right), \eta \in T[p, A] \backslash A$. Let

$$
X=\left\{\rho \in \operatorname{pos}\left(t_{\eta}^{p}\right): \sum_{\ell \leq m} \mu_{p_{\ell}, A^{\ell}}(\rho) \geq \sum_{\ell<n \leq m} \mu_{p_{\ell, n}, A_{\ell, n}}(\rho)\right\}
$$

and $Y=\operatorname{pos}\left(t_{\eta}^{p}\right) \backslash X$. It follows from the inductive hypothesis and 2.1.3(c) that $F_{\eta}\left(\mu_{p, A}(\rho): \rho \in X\right) \geq \sum_{\ell \leq m} F_{\eta}\left(\mu_{p_{\ell}, A^{\ell}}(\rho): \rho \in X\right)-\sum_{\ell<n \leq m} F_{\eta}\left(\mu_{p_{\ell, n}, A_{\ell, n}}(\rho): \rho \in X\right)$.

[Note that though 2.1.3 (c) guarantees the additivity of $F_{\eta}$ only when $r_{\nu}=r_{\nu}^{\prime}+r_{\nu}^{\prime \prime}$, $r_{\nu}, r_{\nu}^{\prime}, r_{\nu}^{\prime \prime} \in[0,1]$, we can first prove that

$$
F_{\eta}\left(\frac{1}{M} \cdot r_{\nu}: \nu \in \operatorname{pos}\left(t_{\eta}^{*}\right)\right)=\frac{1}{M} F_{\eta}\left(r_{\nu}: \nu \in \operatorname{pos}\left(t_{\nu}^{*}\right)\right) .
$$


Next, we may reduce the needed additivity to the one postulated in 2.1.3(c) by dividing all terms by suitably large $M$.] Now, by 2.1.1(e),

$$
\sum_{\ell \leq m} F_{\eta}\left(\mu_{p_{\ell}, A^{\ell}}(\rho): \rho \in Y\right) \leq \sum_{\ell<n \leq m} F_{\eta}\left(\mu_{p_{\ell, n}, A_{\ell, n}}(\rho): \rho \in Y\right),
$$

and hence

$$
F_{\eta}\left(\mu_{p, A}(\rho): \rho \in Y\right) \geq \sum_{\ell \leq m} F_{\eta}\left(\mu_{p_{\ell}, A^{\ell}}(\rho): \rho \in Y\right)-\sum_{\ell<n \leq m} F_{\eta}\left(\mu_{p_{\ell, n}, A_{\ell, n}}(\rho): \rho \in Y\right) .
$$

Since

$$
\begin{aligned}
& \mu_{p, A}(\eta)=F_{\eta}\left(\mu_{p, A}(\rho): \rho \in X\right)+F_{\eta}\left(\mu_{p, A}(\rho): \rho \in Y\right) \\
& \mu_{p_{\ell}, A^{\ell}}(\eta) \\
& \mu_{p_{\ell, n}, A_{\ell, n}}(\eta)=F_{\eta}\left(\mu_{p_{\ell}, A^{\ell}}(\rho): \rho \in X\right)+F_{\eta}\left(\mu_{p_{\ell}, A^{\ell}}(\rho): \rho \in Y\right), \\
& =F_{\eta}\left(\mu_{p_{\ell, n}, A_{\ell, n}}(\rho): \rho \in X\right)+F_{\eta}\left(\mu_{p_{\ell, n}, A_{\ell, n}}(\rho): \rho \in Y\right),
\end{aligned}
$$

we may easily finish.

Now we apply 2.1.4.1 to $\nu=\operatorname{root}\left(p_{0}\right)$. We get then

$$
1 \geq \mu_{p, A}(\nu) \geq \sum_{\ell \leq m} \mu_{p_{\ell}, A^{\ell}}(\nu)-\sum_{\ell<n \leq m} \mu_{p_{\ell, n}, A_{\ell, n}}(\nu)>\sum_{\ell \leq m} \mu^{\mathbf{F}}\left(p_{\ell}\right)-2^{m+1} \cdot \varepsilon=1
$$

a contradiction.

Corollary 2.1.5. Let $(K, \Sigma, \mathbf{F})$ be a ccc-complete mixing triple for $\mathbf{H}$.

1. The forcing notion $\mathbb{Q}_{\emptyset}^{\mathrm{mt}}(K, \Sigma, \mathbf{F})$ satisfies the ccc.

2. If $f: \omega \times \omega \longrightarrow \omega$ is a fast function and $(K, \Sigma)$ is linked, then the forcing notions $\mathbb{Q}_{f}^{\mathrm{mt}}(K, \Sigma, \mathbf{F})$ and $\mathbb{Q}_{1}^{\mathrm{mt}}(K, \Sigma, \mathbf{F})$ are ccc.

3. If $h: \omega \times \omega \longrightarrow \omega$ is regressive, $(K, \Sigma)$ is $h$-linked and $\mathcal{F} \subseteq(\omega \backslash 2)^{\omega}$ is either countable or $\geq^{*}$-directed, then $\mathbb{Q}_{\mathcal{F}}^{\mathrm{mt}}(K, \Sigma, \mathbf{F})$ is ccc.

Remark 2.1.6. 1. Forcing notions determined by mixing triples are in some sense mixtures of the random real forcing with forcings determined by tree-creating pairs. The "mt" in $\mathbb{Q}_{*}^{\mathrm{mt}}(K, \Sigma, \mathbf{F})$ stands for "measured tree".

2. Because of 2.1.5(1) (and the proof of 2.1.4) we can be very generous as far as the demands on the norms are concerned, and still we may easily ensure that the resulting forcing notion satisfies the ccc. For example, if $(K, \Sigma, \mathbf{F})$ is a ccc-complete mixing triple, $(K, \Sigma)$ is semi-linked in the sense that the demand of $1.2 .3(1)$ is satisfied whenever $\operatorname{lh}(\eta)$ is even, and $\mathbb{Q}_{1 / 2}^{\mathrm{mt}}(K, \Sigma, \mathbf{F})=\left\{p \in \mathbb{Q}_{\emptyset}^{\mathrm{mt}}(K, \Sigma, \mathbf{F}):\left(\forall \eta \in\left[T^{p}\right]\right)\left(\lim _{k \rightarrow \infty} \operatorname{nor}\left[t_{\eta \uparrow 2 k}^{p}\right]=\infty\right)\right\}$, then $\mathbb{Q}_{1 / 2}^{\mathrm{mt}}(K, \Sigma, \mathbf{F})$ is ccc too.

3. This type of constructions (i.e., mixture-like) for not-ccc case will be presented in [18] and [17, $\S 2]$.

Let us finish this subsection with showing that the forcing notions $\mathbb{Q}_{\emptyset}^{\mathrm{mt}}(K, \Sigma, \mathbf{F})$ tend to have many features of the random real forcing.

Definition 2.1.7. Let $(K, \Sigma, \mathbf{F})$ be a mixing triple, $p \in \mathbb{Q}_{\emptyset}^{\mathrm{mt}}(K, \Sigma, \mathbf{F})$.

1. A function $\mu: T^{p} \longrightarrow[0,1]$ is a semi $-\mathbf{F}-$ measure on $p$ if

$$
\left(\forall \eta \in T^{p}\right)\left(\mu(\eta) \leq F_{\eta}\left(\mu(\nu): \nu \in \operatorname{pos}\left(t_{\eta}^{p}\right)\right)\right) .
$$

2. If above the equality holds (for each $\eta \in T^{p}$ ), then $\mu$ is called an $\mathbf{F}-$ measure. 
Proposition 2.1.8. Assume $(K, \Sigma, \mathbf{F})$ is a mixing triple, $p \in \mathbb{Q}_{\emptyset}^{\text {tree }}(K, \Sigma)$.

1. If $\mu: T^{p} \longrightarrow[0,1]$ is semi $-\mathbf{F}-$ measure on $p$, then for each $\eta \in T^{p}$ we have $\mu(\eta) \leq \mu^{\mathbf{F}}\left(p^{[\eta]}\right)$.

2. The mapping $\eta \mapsto \mu^{\mathbf{F}}\left(p^{[\eta]}\right): T^{p} \longrightarrow[0,1]$ is an $\mathbf{F}-$ measure on $p$.

3. If there is a semi- $\mathbf{F}$-measure $\mu$ on $p$ such that $\mu(\operatorname{root}(p))>0$, then $p \in$ $\mathbb{Q}_{\emptyset}^{\mathrm{mt}}(K, \Sigma, \mathbf{F})$.

Proof. Straightforward.

Proposition 2.1.9. Suppose that $(K, \Sigma, \mathbf{F})$ is a ccc-complete mixing triple, and $p_{0}, \ldots, p_{m} \in \mathbb{Q}_{\emptyset}^{\mathrm{mt}}(K, \Sigma, \mathbf{F})$ are such that $\operatorname{root}\left(p_{0}\right)=\ldots=\operatorname{root}\left(p_{m}\right)$. Let $p \in$ $\mathbb{Q}_{\emptyset}^{\mathrm{mt}}(K, \Sigma, \mathbf{F})$ be such that $T^{p}=T^{p_{0}} \cup \ldots \cup T^{p_{m}}$. Then:

1. $\mu^{\mathbf{F}}(p) \leq \sum_{\ell \leq m} \mu^{\mathbf{F}}\left(p_{\ell}\right)$,

2. if $\left[T^{p_{\ell}}\right] \cap\left[T^{p_{n}}\right]=\emptyset$ for $\ell<n \leq m$ (or just $p_{0}, \ldots, p_{m}$ are pairwise incompatible in $\left.\mathbb{Q}_{\emptyset}^{\mathrm{mt}}(K, \Sigma, \mathbf{F})\right)$, then $\mu^{\mathbf{F}}(p)=\sum_{\ell \leq m} \mu^{\mathbf{F}}\left(p_{\ell}\right)$,

3. $\left\{p_{0}, \ldots, p_{m}\right\}$ is pre-dense above $p$.

Proof. Like 2.1.4.

Lemma 2.1.10. Let $(K, \Sigma, \mathbf{F})$ be a ccc-complete mixing triple.

1. Suppose that conditions $p, q \in \mathbb{Q}_{\emptyset}^{\mathrm{mt}}(K, \Sigma, \mathbf{F})$ are such that $\operatorname{root}(p)=\operatorname{root}(q)$, $p \leq q, \mu^{\mathbf{F}}(q)<\mu^{\mathbf{F}}(p)$, and let $0<\varepsilon<1$. Then there is a condition $r \in$ $\mathbb{Q}_{\emptyset}^{\mathrm{mt}}(K, \Sigma, \mathbf{F})$ stronger than $p$ and incompatible with $q$ and such that $\operatorname{root}(r)=$ $\operatorname{root}(p), \mu^{\mathbf{F}}(r) \geq(1-\varepsilon) \cdot\left(\mu^{\mathbf{F}}(p)-\mu^{\mathbf{F}}(q)\right)$.

2. If $p \in \mathbb{Q}_{\emptyset}^{\mathrm{mt}}(K, \Sigma, \mathbf{F})$, $\varepsilon>0$ then there is $\eta \in T^{p}$ such that $\mu^{\mathbf{F}}\left(p^{[\eta]}\right)>(1-\varepsilon)$.

Proof. 1) Let $\nu=\operatorname{root}(p)$. Choose a front $A$ of $T^{p}$ such that

$$
\mu_{q, A \cap T^{q}}(\nu)<\mu^{\mathbf{F}}(q)+\varepsilon \cdot\left(\mu^{\mathbf{F}}(p)-\mu^{\mathbf{F}}(q)\right)<\mu^{\mathbf{F}}(p)
$$

(so necessarily $A \backslash T^{q} \neq \emptyset$ ). Take $r_{0}, r_{1} \in \mathbb{Q}_{\emptyset}^{\text {tree }}(K, \Sigma)$ such that

$$
\begin{aligned}
& T^{r_{0}}=\left\{\eta \in T^{p}:\left(\exists \rho \in A \backslash T^{q}\right)(\eta \unlhd \rho \text { or } \rho \triangleleft \eta)\right\} \\
& T^{r_{1}}=\left\{\eta \in T^{p}:\left(\exists \rho \in A \cap T^{q}\right)(\eta \unlhd \rho \text { or } \rho \triangleleft \eta)\right\} .
\end{aligned}
$$

Clearly $\operatorname{root}\left(r_{i}\right)=\nu, A \cap T^{r_{i}}$ is a front of $T^{r_{i}}, A=\left(A \cap T^{r_{0}}\right) \cup\left(A \cap T^{r_{1}}\right)$ and $T^{q} \subseteq T^{r_{1}}$. Hence, $r_{1} \in \mathbb{Q}_{\emptyset}^{\text {mt }}(K, \Sigma, \mathbf{F})$ and $\mu^{\mathbf{F}}(q) \leq \mu^{\mathbf{F}}\left(r_{1}\right)<\mu^{\mathbf{F}}(q)+\varepsilon \cdot\left(\mu^{\mathbf{F}}(p)-\mu^{\mathbf{F}}(q)\right)$. Now, using 2.1.3(c), we may conclude that $\mu^{\mathbf{F}}\left(r_{0}\right) \geq(1-\varepsilon) \cdot\left(\mu^{\mathbf{F}}(p)-\mu^{\mathbf{F}}(q)\right)$, finishing the proof.

2) Straightforward.

Proposition 2.1.11. Assume that $(K, \Sigma, \mathbf{F})$ is a ccc-complete mixing triple, $p \in$ $\mathbb{Q}_{\emptyset}^{\mathrm{mt}}(K, \Sigma, \mathbf{F})$. Let $m<\omega, \varepsilon>0$ and let $\dot{\tau}$ be a $\mathbb{Q}_{\emptyset}^{\mathrm{mt}}(K, \Sigma, \mathbf{F})$-name such that $p \Vdash \dot{\tau}<m$. Then there are $X \subseteq m$ and conditions $q_{\ell} \in \mathbb{Q}_{\emptyset}^{\mathrm{mt}}(K, \Sigma, \mathbf{F})$ (for $\ell \in X$ ) such that

( $\alpha) q_{\ell} \Vdash \dot{\tau}=\ell$,

( $\beta) \operatorname{root}\left(q_{\ell}\right)=\operatorname{root}(p)$,

$(\gamma) \sum_{\ell \in X} \mu^{\mathbf{F}}\left(q_{\ell}\right) \geq(1-\varepsilon) \mu^{\mathbf{F}}(p)$. 
Proof. Let $\nu=\operatorname{root}(p)$. For each $\ell<m$ define $\mu_{\ell}: T^{p} \longrightarrow[0,1]$ by:

$$
\mu_{\ell}(\eta)=\sup \left\{\mu^{\mathbf{F}}(q): q \in \mathbb{Q}_{\emptyset}^{\mathrm{mt}}(K, \Sigma, \mathbf{F}) \& q \geq p^{[\eta]} \& \operatorname{root}(q)=\eta \& q \Vdash \dot{\tau}=\ell\right\}
$$

(with the convention that $\sup \emptyset=0$ ). It follows from 2.1.1(f) that each $\mu_{\ell}$ is an $\mathbf{F}$-measure on $p$.

Claim 2.1.11.1. $\mu^{\mathbf{F}}(p)=\sum_{\ell<m} \mu_{\ell}(\nu)$.

Proof of the claim. First note that, by a suitable modification of 2.1.4, we have $\mu^{\mathbf{F}}(p) \geq \sum_{\ell<m} \mu_{\ell}(\nu)$. So suppose that $\mu^{\mathbf{F}}(p)>\sum_{\ell<m} \mu_{\ell}(\nu)$, and let $n \in \omega$ be such that $\left(1-\frac{2}{n}\right) \cdot \mu^{\mathbf{F}}(p)>\sum_{\ell<m} \mu_{\ell}(\nu)$. Let

$$
S=\left\{\eta \in T^{p}:\left(1-\frac{1}{n}\right) \cdot \mu^{\mathbf{F}}\left(p^{[\eta]}\right)>\sum_{\ell<m} \mu_{\ell}(\eta)\right\},
$$

and let $T \subseteq S$ be a tree such that $\operatorname{root}(T)=\nu$ and $\eta \in T, \rho \in \operatorname{succ}_{T^{p}}(\eta) \cap S$ imply $\rho \in T$. Clearly $\max (T)=\emptyset$, so we may choose $r \in \mathbb{Q}_{\emptyset}^{\text {tree }}(K, \Sigma)$ so that $T=T^{r}$. If $\mu^{\mathbf{F}}(r)>0$, then $\left(r \in \mathbb{Q}_{\emptyset}^{\mathrm{mt}}(K, \Sigma, \mathbf{F})\right.$ and $)$ we may choose a condition $q \in \mathbb{Q}_{\emptyset}^{\mathrm{mt}}(K, \Sigma, \mathbf{F})$ stronger than $r$ which decides the value of $\dot{\tau}$. By 2.1.10 (2) we find $\eta \in T^{q}$ such that $\mu^{\mathbf{F}}\left(q^{[\eta]}\right)>\left(1-\frac{1}{n}\right) \geq\left(1-\frac{1}{n}\right) \mu^{\mathbf{F}}\left(p^{[\eta]}\right)$, what contradicts $T^{q} \subseteq T^{r} \subseteq S$. Therefore $\mu^{\mathbf{F}}(r)=0$, and like in 2.1.10 (1) we may build a condition $q \geq p$ and a front $A$ of $T^{q}$ such that

- $\operatorname{root}(q)=\nu, A \cap T^{r}=\emptyset$,

- $T^{q}=\left\{\eta \in T^{p}:(\exists \rho \in A)(\eta \unlhd \rho\right.$ or $\left.\rho \triangleleft \eta)\right\}$,

- $\left(1-\frac{1}{n}\right) \mu^{\mathbf{F}}\left(p^{[\eta]}\right) \leq \sum_{\ell<m} \mu_{\ell}(\eta) \leq \mu^{\mathbf{F}}\left(p^{[\eta]}\right)$ for each $\eta \in A$, and

- $\mu^{\mathbf{F}}(q)>\left(1-\frac{1}{n}\right) \mu^{\mathbf{F}}(p)$.

Now, we may easily conclude that $\sum_{\ell<m} \mu_{\ell}(\nu) \geq\left(1-\frac{2}{n}\right) \cdot \mu^{\mathbf{F}}(p)$, getting a contradiction.

The conclusion of the proposition follows immediately from 2.1.11.1 and the definition of $\mu_{\ell}$ 's (so we take $X=\left\{\ell<m: \mu_{\ell}(\nu)>0\right\}$ and suitable $q_{\ell}$ 's for $\ell \in X$ ).

Proposition 2.1.12. Suppose that $(K, \Sigma, \mathbf{F})$ is a ccc-complete mixing triple and $\dot{\tau}$ is $a \mathbb{Q}_{\emptyset}^{\mathrm{mt}}(K, \Sigma, \mathbf{F})$-name for an ordinal. Let $p \in \mathbb{Q}_{\emptyset}^{\mathrm{mt}}(K, \Sigma, \mathbf{F}), 0<\varepsilon<1$. Then there is a condition $q \geq p$ and a front $A$ of $T^{q}$ such that

$(\alpha) \operatorname{root}(q)=\operatorname{root}(p), \mu^{\mathbf{F}}(q)>(1-\varepsilon) \mu^{\mathbf{F}}(p)$,

( $\beta)$ for each $\eta \in A$ the condition $q^{[\eta]}$ decides the value of $\dot{\tau}$.

Proof. Let

$$
\begin{aligned}
B=\left\{\eta \in T^{p}:\right. & \text { for some } p^{*} \geq p \text { we have: } \operatorname{root}\left(p^{*}\right)=\eta, \\
& \left.\mu^{\mathbf{F}}\left(p^{*}\right) \geq\left(1-\frac{\varepsilon}{2}\right) \mu^{\mathbf{F}}\left(p^{[\eta]}\right), \quad \text { and } p^{*} \text { decides } \dot{\tau} \text { on a front }\right\} .
\end{aligned}
$$

It follows from 2.1 .10 that, if $q \in \mathbb{Q}_{\emptyset}^{\mathrm{mt}}(K, \Sigma, \mathbf{F})$ is a condition stronger than $p$, then $T^{q} \cap B \neq \emptyset$. If $\operatorname{root}(p) \in B$, then we are clearly done, so suppose $\operatorname{root}(p) \notin B$. Note that if $\eta \in T^{p} \backslash B$ then $\operatorname{succ}_{T^{p}}(\eta) \backslash B \neq \emptyset$. Thus

$$
T \stackrel{\text { def }}{=}\left\{\eta \in T^{p}:(\forall \nu \unlhd \eta)(\nu \notin B)\right\}
$$


is a tree with $\max (T)=\emptyset, T \cap B=\emptyset, \operatorname{root}(T)=\operatorname{root}(p)$. This $T$ determines a condition $r \in \mathbb{Q}_{\emptyset}^{\text {tree }}(K, \Sigma), \operatorname{root}(r)=\operatorname{root}(p)$. It follows from the previous remark that $\mu^{\mathbf{F}}(r)=0$. Take a front $A$ of $T^{p}$ such that $\mu_{r, A \cap T^{r}}(\operatorname{root}(r))<\frac{1}{4} \cdot \varepsilon \cdot \mu^{\mathbf{F}}(p)$ and $A \subseteq T^{r} \cup B$. For each $\nu \in A \backslash T^{r}$ fix a condition $q_{\nu}$ such that $p^{[\nu]} \leq q_{\nu}$, $\mu^{\mathbf{F}}\left(q_{\nu}\right) \geq\left(1-\frac{\varepsilon}{2}\right) \mu^{\mathbf{F}}\left(p^{[\nu]}\right)$, and $q_{\nu}$ decides $\dot{\tau}$ on a front. Let $q$ be such that

$$
T^{q}=\left\{\eta \in T^{p}:\left(\exists \nu \in A \backslash T^{r}\right)\left(\eta \in T^{q_{\nu}} \text { or } \nu \unlhd \eta\right)\right\} .
$$

It should be clear that $q$ is a condition as required

2.2. Exotic norm conditions. The norm conditions introduced in the first section have their counterparts in the non-ccc case (as presented in [15]). Here we formulate more norm conditions which may be used to build ccc forcing notions from linked creating pairs (or tree-creating pairs), and which seem to be very cccspecific. Let us start with a norm condition that allows us to include into our framework the "Mathias with ultrafilter" forcing notion.

Definition 2.2.1. $\quad$ 1. A local creating pair $(K, \Sigma)$ for $\mathbf{H}$ is strongly linked if it is full (see 1.1.2 (5)), linked and

$(\otimes)^{\mathrm{sl}}$ there are $t_{\ell}^{\min } \in K($ for $\ell<\omega)$ such that $m_{\mathrm{dn}}^{t_{\ell}^{\min }}=\ell$ and if $t \in K, m_{\mathrm{dn}}^{t}=\ell$, then $t_{\ell}^{\min } \in \Sigma(t)$.

If additionally for each $\ell<\omega$ we have

$$
\left(\forall u \in \operatorname{dom}\left(\operatorname{val}\left[t_{\ell}^{\min }\right]\right)\right)\left(\exists ! v \in \operatorname{rng}\left(\operatorname{val}\left[t_{\ell}^{\min }\right]\right)\right)(u \triangleleft v),
$$

then we say that $(K, \Sigma)$ is strongly ${ }^{+}$linked.

2. A local tree-creating pair $(K, \Sigma)$ for $\mathbf{H}$ is (tree-) strongly linked if it is linked and

$(\otimes)_{\text {tree }}^{\text {sl }}$ there are $t_{\eta}^{\text {min }} \in K \cap \operatorname{LTCR}_{\eta}[\mathbf{H}]$ (for $\eta \in \bigcup_{m \in \omega} \prod_{i<m} \mathbf{H}(i)$ ) such that if $t \in K \cap \operatorname{LTCR}_{\eta}[\mathbf{H}], \eta \in \bigcup_{m \in \omega} \prod_{i<m} \mathbf{H}(i)$, then $t_{\eta}^{\min } \in \Sigma(t)$.

If, additionally, $\left|\operatorname{pos}\left(t_{\eta}^{\min }\right)\right|=1$ for each $\eta$ then we say that $(K, \Sigma)$ is strongly ${ }^{+}$ linked.

3. Let $D$ be a non-principal ultrafilter on $\omega$. We define norm conditions $\mathcal{C}(D)$ and $\mathcal{C}^{\text {tree }}(D)$ (for the contexts of creating pairs and tree-creating pairs, respectively) and the corresponding forcing notions $\mathbb{Q}_{D}^{*}(K, \Sigma), \mathbb{Q}_{D}^{\text {tree }}(K, \Sigma)$ as follows.

- A sequence $\left\langle t_{i}: i<\omega\right\rangle$ satisfies $\mathcal{C}(D)$ if for some $\ell<\omega$ we have:

$(\forall i<\omega)\left(m_{\mathrm{dn}}^{t_{i}}=\ell+i\right) \quad$ and $\quad \lim _{D}\left\langle\operatorname{nor}\left[t_{j-\ell}\right]: \ell \leq j<\omega\right\rangle=\infty$.

For a local creating pair $(K, \Sigma), \mathbb{Q}_{D}^{*}(K, \Sigma)$ is the forcing notion

$$
\mathbb{Q}_{\mathcal{C}(D)}^{*}(K, \Sigma)=\left\{p \in \mathbb{Q}_{\emptyset}^{*}(K, \Sigma):\left\langle t_{i}^{p}: i<\omega\right\rangle \text { satisfies } \mathcal{C}(D)\right\} .
$$

- A system $\left\langle t_{\eta}: \eta \in T\right\rangle \subseteq \operatorname{LTCR}[\mathbf{H}]$ satisfies $\mathcal{C}^{\text {tree }}(D)$ if $T$ is a tree, $t_{\eta} \in \operatorname{LTCR}_{\eta}[\mathbf{H}]$ and $\operatorname{pos}\left(t_{\eta}\right)=\operatorname{succ}_{T}(\eta) \subseteq \prod_{i \leq \operatorname{lh}(\eta)} \mathbf{H}(i)$ for each $\eta \in T$, and $(\forall \eta \in[T])\left(\lim _{D}\left\langle\operatorname{nor}\left[t_{\eta \uparrow k}\right]: \operatorname{lh}(\operatorname{root}(T)) \leq k<\omega\right\rangle=\infty\right)$.

For a local tree creating pair $(K, \Sigma), \mathbb{Q}_{D}^{\text {tree }}(K, \Sigma)$ is the forcing notion $\mathbb{Q}_{\mathcal{C}(D)}^{\text {tree }}(K, \Sigma)=\left\{p \in \mathbb{Q}_{\emptyset}^{\text {tree }}(K, \Sigma):\left\langle t_{\eta}^{p}: \eta \in T^{p}\right\rangle\right.$ satisfies $\left.\mathcal{C}^{\text {tree }}(D)\right\}$. 
Remark 2.2.2. Strongly linked (and especially strongly ${ }^{+}$linked) creating pairs resemble omittory creating pairs of [15, Def. 2.1.1] - in both cases the practical examples are such that we may "omit" some of the creatures from a condition $p$. Here the "omitting" is done by replacing $t_{i}^{p}$ by the suitable $t_{\ell}^{\min }$ (see 2.4.5).

Proposition 2.2.3. Let $D$ be a non-principal ultrafilter on $\omega$. If $(K, \Sigma)$ is a local strongly linked creating pair (tree-creating pair, respectively), then the forcing notion $\mathbb{Q}_{D}^{*}(K, \Sigma)\left(\mathbb{Q}_{D}^{\text {tree }}(K, \Sigma)\right.$, respectively) is $\sigma$-centered.

Proof. Straightforward.

Forcing notions $\mathbb{Q}_{D}^{*}(K, \Sigma)$, though similar to the Mathias forcing notion, do not have (in general) as many nice properties as this one. For example "deciding formulas without changing the root" may easily fail, even though we may have some kind of continuous reading of names.

Definition 2.2.4 (See [15, Def. 1.2.9]). Let $D$ be a non-principal ultrafilter on $\omega$, $(K, \Sigma)$ be a local strongly linked creating pair and $\dot{\tau}$ be a $\mathbb{Q}_{D}^{*}(K, \Sigma)$-name for an ordinal. We say that a condition $p \in \mathbb{Q}_{D}^{*}(K, \Sigma)$ approximates $\dot{\tau}$ at $t_{n}^{p}$ (or at $n$ ) whenever the following demand is satisfied:

(*) for each $w_{1} \in \operatorname{pos}\left(w^{p}, t_{0}^{p}, \ldots, t_{n-1}^{p}\right)$, if there is a condition $r \in \mathbb{Q}_{D}^{*}(K, \Sigma)$ stronger than $p$ and such that $w^{r}=w_{1}$ and $r$ decides the value of $\dot{\tau}$, then the condition $\left(w_{1}, t_{n}^{p}, t_{n+1}^{p}, \ldots\right)$ decides the value of $\dot{\tau}$

Proposition 2.2.5. Assume that $D$ is a Ramsey ultrafilter on $\omega$ and $(K, \Sigma)$ is a local, really finitary and strongly ${ }^{+}$linked creating pair. Then for each $p \in \mathbb{Q}_{D}^{*}(K, \Sigma)$ and a name $\dot{\tau}$ for an ordinal, there is a condition $q \geq p$ which approximates $\dot{\tau}$ at every $n$ and such that $w^{p}=w^{q}$.

Proof. The proof follows the lines of the appropriate proof for the Mathias forcing notion (see e.g. [1], §7.4]). Let $\left\langle t_{\ell}^{\min }: \ell<\omega\right\rangle$ witness that $(K, \Sigma)$ satisfies $(\otimes)^{\text {sl }}$ of $2.2 .1(1),\left(\forall u \in \operatorname{dom}\left(\operatorname{val}\left[t_{\ell}^{\min }\right]\right)\right)\left(\exists ! v \in \operatorname{rng}\left(\operatorname{val}\left[t_{\ell}^{\min }\right]\right)\right)(u \triangleleft v)$. For simplicity, we assume that nor $\left[t_{\ell}^{\min }\right] \leq 1$ (for $\left.\ell<\omega\right)$.

For a condition $p \in \mathbb{Q}_{D}^{*}(K, \Sigma)$ and $n \in \omega$ let

$$
\operatorname{supp}^{n}(p) \stackrel{\text { def }}{=}\left\{m_{\mathrm{dn}}^{t_{i}^{p}}: i<\omega \& \operatorname{nor}\left[t_{i}^{p}\right]>n+1\right\} \in D .
$$

Choose inductively conditions $p_{n} \in \mathbb{Q}_{D}^{*}(K, \Sigma)$ such that for each $n<\omega$ :

1. $p_{0}=p, p_{n} \leq p_{n+1}, w^{p_{n}}=w^{p}$, and $t_{i}^{p_{n+1}}=t_{i}^{p_{n}}$ for $i<n$,

2. if $w \in \operatorname{pos}\left(w^{p}, t_{0}^{p}, \ldots, t_{n-1}^{p}\right)$ and there is $p^{*} \geq p$ such that $w^{p^{*}}=w$ and $p^{*}$ decides $\dot{\tau}$, then $\left(w, t_{n}^{p_{n+1}}, t_{n+1}^{p_{n+1}}, \ldots\right)$ decides $\dot{\tau}$.

(Note that "strongly linked" implies that if $w^{q_{0}}=w^{q_{1}}$, then $q_{0}, q_{1}$ are compatible; also remember that $(K, \Sigma)$ is full.) Since $D$ is Ramsey, we may choose an increasing sequence $\left\langle i_{n}: n<\omega\right\rangle \subseteq \omega \backslash \ln \left(w^{p}\right)$ such that

$$
\left\{i_{n}: n<\omega\right\} \in D \quad \text { and } \quad(\forall n \in \omega)\left(i_{n}+2<i_{n+1} \in \operatorname{supp}^{n+1}\left(p_{i_{n}-\operatorname{lh}\left(w^{p}\right)+2}\right)\right) .
$$

For $j<\omega$ let

$$
t_{j}^{q}= \begin{cases}t_{j}^{p_{i_{n}-\mathrm{lh}\left(w^{p}\right)+2}} & \text { if } j=i_{n+1}-\operatorname{lh}\left(w^{p}\right), n \in \omega \\ t_{j}^{\min } & \text { if } j+\ln \left(w^{p}\right) \notin\left\{i_{n+1}: n<\omega\right\} .\end{cases}
$$


It should be clear that $q \stackrel{\text { def }}{=}\left(w^{p}, t_{0}^{q}, t_{1}^{q}, \ldots\right) \in \mathbb{Q}_{D}^{*}(K, \Sigma)$ is a condition stronger than $p$ and for every $w \in \operatorname{pos}\left(w^{p}, t_{0}^{p}, \ldots, t_{i_{n}-\operatorname{lh}\left(w^{p}\right)}^{p}\right), n \in \omega$ we have

$$
\left(w, t_{i_{n}-\operatorname{lh}\left(w^{p}\right)+1}^{q}, t_{i_{n}-\operatorname{lh}\left(w^{p}\right)+2}^{q}, \ldots\right) \geq\left(w, t_{i_{n}-\operatorname{lh}\left(w^{p}\right)+1}^{p_{i_{n}-\ln \left(p^{p}\right)+2}}, t_{i_{n}-\operatorname{lh}\left(w^{p}\right)+2}^{p_{i_{n}-\ln \left(w^{p}\right)+2}}, \ldots\right) .
$$

Hence easily $q$ approximates $\dot{\tau}$ at all points of the form $i_{n+1}-\operatorname{lh}\left(w^{p}\right)+1$ (for $n<\omega$ ), and by the additional demand on $t_{\ell}^{\min }$ (in "strongly") ${ }^{+}$) we conclude that $q$ approximates $\dot{\tau}$ at all $n<\omega$.

Proposition 2.2.6. Suppose that $(K, \Sigma)$ is a strongly linked local creating pair (with $t_{n}^{\mathrm{min}}$ witnessing this). Assume that $\operatorname{nor}\left[t_{n}^{\mathrm{min}}\right] \leq 1$ (for $\left.n<\omega\right)$ and

$(*)$ for each $n \in \omega$ there are disjoint sets $A_{n}, B_{n} \subseteq \mathbf{H}(n)$ such that

- if $\langle u, v\rangle \in \operatorname{val}\left[t_{n}^{\min }\right]$, then $v(n) \notin A_{n} \cup B_{n}$,

- if $t \in K, \operatorname{nor}[t]>1, u \in \operatorname{dom}(\operatorname{val}[t]), \operatorname{lh}(u)=n$

then there are $v_{0}, v_{1}$ such that $\left\langle u, v_{0}\right\rangle,\left\langle u, v_{1}\right\rangle \in \mathbf{v a l}[t]$ and $v_{1}(n) \in A_{n}$ and $v_{0}(n) \in B_{n}$.

Let $D$ be an ultrafilter on $\omega$. Then the forcing notion $\mathbb{Q}_{D}^{*}(K, \Sigma)$ adds a Cohen real.

Proof. Let $\dot{W}$ be the name for $\mathbb{Q}_{D}^{*}(K, \Sigma)$-generic real and let $\dot{K}=\left\{\dot{k}_{n}: n<\omega\right\}$ be a name for a subset of $\omega$ such that

$$
\dot{K}=\left\{k \in \omega: \dot{W}(k) \in A_{k} \cup B_{k}\right\} .
$$

(Clearly $\dot{K}$ is infinite.) Let $\dot{c} \in 2^{\omega}$ be given by $\dot{c}(n)=0$ if and only if $\dot{W}\left(\dot{k}_{n}\right) \in A_{n}$. It should be clear that $\dot{c}$ is a name for a Cohen real over $\mathbf{V}$.

Now we will give some norm conditions that can be used in the context of local and forgetful creating pairs. Note that if $(K, \Sigma)$ is of that type, then for each $t \in K$ we have (unique) set $P_{t} \subseteq \mathbf{H}\left(m_{\mathrm{dn}}^{t}\right)$ such that

$$
\langle u, v\rangle \in \operatorname{val}[t] \quad \text { if and only if } \quad v\left(m_{\mathrm{dn}}^{t}\right) \in P_{t}, u \triangleleft v \text { and } \operatorname{lh}(v)=\operatorname{lh}(u)+1
$$

(for some, equivalently all, $u \in \operatorname{dom}(\operatorname{val}[t])$ ). The set $P_{t}$ corresponds to $\operatorname{pos}(t)$ in the tree-creatures context, and below we will use the notation $\operatorname{pos}(t)$ for it (hoping that this does not cause any confusion). Our next definition is a variant of 2.1.3 (a,b) for the case of local forgetful creating pairs.

Definition 2.2.7. A local forgetful creating pair $(K, \Sigma)$ for $\mathbf{H}$ is complete if

(a) for each $i \in \omega$ and a nonempty set $A \subseteq \mathbf{H}(i)$, there is a unique creature $t_{A}^{i} \in K$ such that $m_{\mathrm{dn}}^{t_{A}^{i}}=i$ and $\operatorname{pos}\left(t_{A}^{i}\right)=A$,

(b) if $i \in \omega, A \subseteq B \subseteq \mathbf{H}(i)$, then $t_{A}^{i} \in \Sigma\left(t_{B}^{i}\right)$ and $\operatorname{nor}\left[t_{A}^{i}\right] \leq \operatorname{nor}\left[t_{B}^{i}\right]$.

Definition 2.2.8. Let $\mathbf{H}: \omega \longrightarrow \mathcal{H}\left(\omega_{1}\right)$ be such that $(\forall n \in \omega)\left(|\mathbf{H}(n)|>2^{n}\right)$ and let $(K, \Sigma)$ be a complete pair for $\mathbf{H}$.

1. A 1-norming system (for $\mathbf{H}$ ) is a pair $(\bar{K}, \bar{g})$ such that

$(\alpha) \bar{K}=\left\langle K_{\ell}: \ell \in \omega\right\rangle$ is a sequence of infinite pairwise disjoint subsets of $\omega$, $\min \left(K_{\ell}\right) \geq \ell$

( $\beta) \bar{g}=\left\langle g_{\rho}: \rho \in 2^{<\omega}\right\rangle$, where for each $\ell<\omega$ :

$(\gamma)$ if $\rho \in 2^{\ell}$ then $g_{\rho} \in \prod_{m \in K_{\ell}} \mathbf{H}(m)$, and

$(\delta)$ for every $m \in K_{\ell}$, there are no repetitions in $\left\langle g_{\rho}(m): \rho \in 2^{\ell}\right\rangle$. 
2. Let $\mathcal{C}($ nor $)$ be a norm condition for $K$ and $(\bar{K}, \bar{g})$ be a 1 -norming system. We define $(\bar{K}, \bar{g})$-modified version $\mathcal{C}(\text { nor })^{\bar{K}, \bar{g}}$ of $\mathcal{C}($ nor $)$ by a sequence $\left\langle t_{i}: i<\omega\right\rangle$ satisfies $\mathcal{C}(\text { nor })^{\bar{K}, \bar{g}}$ if and only if it satisfies $\mathcal{C}($ nor $)$ and for some $\rho_{0}, \ldots, \rho_{k} \in 2^{\omega}, k<\omega$ we have

$(\forall i, \ell<\omega)\left(\forall \rho \in 2^{\ell}\right)\left(\left[m_{\mathrm{dn}}^{t_{i}} \in K_{\ell} \& g_{\rho}\left(m_{\mathrm{dn}}^{t_{i}}\right) \notin \operatorname{pos}\left(t_{i}\right)\right] \Rightarrow\left[\rho \triangleleft \rho_{0} \vee \ldots \vee \rho \triangleleft \rho_{k}\right]\right)$.

3. If $\mathcal{C}$ (nor) is one of $\mathcal{C}^{\infty}($ nor $), \mathcal{C}^{\mathcal{F}}$ (nor) or $\mathcal{C}^{f}$ (nor) (for suitable $f, \mathcal{F}$; see 1.1.5), then the forcing notions corresponding to $(\bar{K}, \bar{g})$-modified versions of $\mathcal{\mathcal { C }}(\mathbf{n o r})$ will be denoted by $\mathbb{Q}_{\infty}^{\bar{K}, \bar{g}}(K, \Sigma), \mathbb{Q}_{\mathcal{F}}^{\bar{K}, \bar{g}}(K, \Sigma), \mathbb{Q}_{f}^{\bar{K}, \bar{g}}(K, \Sigma)$, respectively.

Proposition 2.2.9. Let $\mathbf{H}: \omega \longrightarrow \mathcal{H}\left(\omega_{1}\right)$ be such that $(\forall n \in \omega)\left(|\mathbf{H}(n)|>2^{n}\right)$. Assume that $(K, \Sigma)$ is a complete creating pair, and $(\bar{K}, \bar{g})$ is a 1 -norming system $($ for $\mathbf{H})$.

1. If $f: \omega \times \omega \longrightarrow \omega$ is fast and $(K, \Sigma)$ is linked, then $\mathbb{Q}_{\infty}^{\bar{K}, \bar{g}}(K, \Sigma)$ and $\mathbb{Q}_{f}^{\bar{K}, \bar{g}}(K, \Sigma)$ are $\sigma$-*-linked Souslin forcing notions.

2. Assume that $h: \omega \times \omega \longrightarrow \omega$ is regressive and $\mathcal{F} \subseteq(\omega \backslash 2)^{\omega}$ is an $h$-closed family which is either countable or $\geq^{*}$-directed. Suppose $(K, \Sigma)$ is local and $h$-linked. Then the forcing notion $\mathbb{Q}_{\mathcal{F}}^{\bar{K}, \bar{g}}(K, \Sigma)$ is $\sigma$-*-linked, and if $\mathcal{F}$ is countable and $\geq^{*}$-directed, then $\mathbb{Q}_{\mathcal{F}}^{\bar{K}, \bar{g}}(K, \Sigma)$ is also Souslin.

Proof. Straightforward.

Definition 2.2.10. Let $(K, \Sigma)$ be a local forgetful creating pair for $\mathbf{H}$.

1. A 2-norming system is a sequence $\bar{U}=\left\langle U_{\rho, k}: \rho \in 2^{<\omega} \& k<\omega\right\rangle$ of pairwise disjoint infinite subsets of $\omega$ such that $\ln (\rho) \leq \min \left(U_{\rho, k}\right)$.

2. For a norm condition $\mathcal{C}$ (nor) and a 2 -norming system $\bar{U}$ we define $\bar{U}$-modified version $\mathcal{C}(\text { nor })^{\bar{U}}$ of $\mathcal{C}($ nor $)$ by

a sequence $\left\langle t_{i}: i<\omega\right\rangle$ satisfies $\mathcal{C}(\text { nor })^{\bar{U}}$ if and only if it satisfies $\mathcal{C}$ (nor) and for some $\rho_{0}, \ldots, \rho_{\ell} \in 2^{\omega}$ and $k_{0}, \ldots, k_{\ell}<\omega, \ell<\omega$, for every $i, k<\omega$ and $\rho \in 2^{<\omega}$ we have:

$\left[m_{\mathrm{dn}}^{t_{i}} \in U_{\rho, k} \& \operatorname{pos}\left(t_{i}\right) \neq \mathbf{H}\left(m_{\mathrm{dn}}^{t_{i}}\right)\right] \Rightarrow\left[\rho \triangleleft \rho_{0} \vee \ldots \vee \rho \triangleleft \rho_{\ell}\right.$ and $\left.k \in\left\{k_{0}, \ldots, k_{\ell}\right\}\right]$.

We will use notation $\mathbb{Q}_{\infty}^{\bar{U}}(K, \Sigma), \mathbb{Q}_{\mathcal{F}}^{\bar{U}}(K, \Sigma), \mathbb{Q}_{f}^{\bar{U}}(K, \Sigma)$ for the respective forcing notions (and suitable $f, \mathcal{F}$ ).

Proposition 2.2.11. Let $(K, \Sigma)$ be a complete creating pair, and $\bar{U}$ be a $2-n o r m i n g$ system.

1. If $f: \omega \times \omega \longrightarrow \omega$ is fast and $(K, \Sigma)$ is linked, then $\mathbb{Q}_{\infty}^{\bar{U}}(K, \Sigma)$ and $\mathbb{Q}_{f}^{\bar{U}}(K, \Sigma)$ are $\sigma-*$-linked Souslin forcing notions.

2. Assume that $h: \omega \times \omega \longrightarrow \omega$ is regressive and $\mathcal{F} \subseteq(\omega \backslash 2)^{\omega}$ is an $h$-closed family which is either countable or $\geq^{*}$-directed. Suppose $(K, \Sigma)$ is local and $h-$ linked. Then the forcing notion $\mathbb{Q}_{\mathcal{F}}^{\bar{U}}(K, \Sigma)$ is $\sigma$-*-linked, and if $\mathcal{F}$ is countable and $\geq^{*}$-directed, then $\mathbb{Q}_{\mathcal{F}}^{\bar{U}}(K, \Sigma)$ is also Souslin.

Proof. Straightforward. 
2.3. Universal forcing notions. Here we introduce constructions involving very peculiar norm conditions. As a matter of fact, norms are not important in that type of constructions, but they still provide examples. Prototypes for the method described here are the Universal Meager forcing notions (i.e., the Amoeba for Category) and forcing notions related to variants of the PP-property (see [28, Ch.VI, $\S 2.12]$, 15, Ch.7]).

Definition 2.3.1. Let $(K, \Sigma)$ be a tree-creating pair for $\mathbf{H}$. A finite candidate for $(K, \Sigma)$ is a system $\left\langle t_{\eta}: \eta \in \hat{S}\right\rangle$ such that

(i) $S \subseteq \bigcup_{k \leq \operatorname{lev}(S)} \prod_{i<k} \mathbf{H}(i)$ is a tree of height $\operatorname{lev}(S)<\omega$, each node in $S$ has a successor at the last level $\operatorname{lev}(S)$,

(ii) $\hat{S}=S \backslash \max (S)$ (i.e., non-maximal nodes of $S$ ),

(iii) $t_{\eta} \in \operatorname{LTCR}_{\eta}[\mathbf{H}] \cap K$ and $\operatorname{pos}\left(t_{\eta}\right)=\operatorname{succ}_{S}(\eta)($ for $\eta \in \hat{S})$.

The collection of finite candidates for $(K, \Sigma)$ is denoted $\mathrm{FC}(K, \Sigma)$. It is equipped with the following order (similar to that of $\mathbb{Q}_{\emptyset}^{\text {tree }}(K, \Sigma)$ ):

$\left\langle t_{\eta}^{0}: \eta \in \hat{S}^{0}\right\rangle \leq\left\langle t_{\eta}^{1}: \eta \in \hat{S}^{1}\right\rangle \quad$ if and only if $\operatorname{lev}\left(S^{0}\right) \leq \operatorname{lev}\left(S^{1}\right)$ and

$$
\left(\forall \eta \in S^{1}\right)\left(\ln (\eta)<\operatorname{lev}\left(S^{0}\right) \quad \Rightarrow \quad \eta \in S^{0} \& t_{\eta}^{1} \in \Sigma\left(t_{\eta}^{0}\right)\right) .
$$

Remark 2.3.2. Finite candidates for tree-creating pairs correspond to that for creating pairs (see 1.1.9). In general, finite candidates do not have to be finite (just the respective tree is of finite height), but if $(K, \Sigma)$ is finitary then they are.

Definition 2.3.3. Let $\mathbf{H}: \omega \longrightarrow \mathcal{H}\left(\omega_{1}\right)$. A universality parameter $\mathfrak{p}$ for $\mathbf{H}$ is a tuple $\left(K^{\mathfrak{p}}, \Sigma^{\mathfrak{p}}, \mathcal{F}^{\mathfrak{p}}, \mathcal{G}^{\mathfrak{p}}\right)=(K, \Sigma, \mathcal{F}, \mathcal{G})$ such that

$(\alpha)(K, \Sigma)$ is a really finitary local tree-creating pair for $\mathbf{H}$,

( $\beta) \mathcal{F} \subseteq \omega^{\omega}$ is either countable or $\leq^{*}$-directed [note the direction of the inequality!],

$(\gamma)$ elements of $\mathcal{G}$ are quadruples $\left(\left\langle t_{\eta}: \eta \in \hat{S}\right\rangle, n_{\mathrm{dn}}, n_{\mathrm{up}}, \bar{r}\right)$ such that

- $\left\langle t_{\eta}: \eta \in \hat{S}\right\rangle \in \mathrm{FC}(K, \Sigma)$,

- $n_{\mathrm{dn}} \leq n_{\mathrm{up}} \leq \operatorname{lev}(S)$,

$(\delta)$ if:

- $\bar{r}=\left\langle r_{i}: i \in \operatorname{dom}(\bar{r})\right\rangle, r_{i} \in \omega, \operatorname{dom}(\bar{r}) \subseteq\left[n_{\mathrm{dn}}, n_{\mathrm{up}}\right]$,

- $\left(\left\langle t_{\eta}^{0}: \eta \in \hat{S}^{0}\right\rangle, n_{\mathrm{dn}}^{0}, n_{\mathrm{up}}^{0}, \bar{r}^{0}\right) \in \mathcal{G}$,

- $\bar{r}^{1}=\left\langle r_{i}^{1}: i \in \operatorname{dom}\left(\bar{r}^{1}\right)\right\rangle, r_{i}^{1} \in \omega, \operatorname{dom}\left(\bar{r}^{0}\right) \subseteq \operatorname{dom}\left(\bar{r}^{1}\right)$, and $r_{i}^{0} \leq r_{i}^{1}$ for $i \in \operatorname{dom}\left(\bar{r}^{0}\right)$, and

- $\left\langle t_{\eta}^{1}: \eta \in \hat{S}^{1}\right\rangle \in \mathrm{FC}(K, \Sigma),\left\langle t_{\eta}^{0}: \eta \in \hat{S}^{0}\right\rangle \leq\left\langle t_{\eta}^{1}: \eta \in \hat{S}^{1}\right\rangle$ and

- $n_{\mathrm{dn}}^{1} \leq n_{\mathrm{dn}}^{0}, n_{\mathrm{up}}^{0} \leq n_{\mathrm{up}}^{1} \leq \operatorname{lev}\left(S^{1}\right)$ and $\operatorname{dom}\left(\bar{r}^{1}\right) \subseteq\left[n_{\mathrm{dn}}^{1}, n_{\mathrm{up}}^{1}\right]$,

then: $\quad\left(\left\langle t_{\eta}^{1}: \eta \in \hat{S}^{1}\right\rangle, n_{\mathrm{dn}}^{1}, n_{\mathrm{up}}^{1}, \bar{r}^{1}\right) \in \mathcal{G}$,

$(\varepsilon)$ for some increasing function $F=F^{\mathcal{G}} \in \omega^{\omega}$, if:

- $\left(\left\langle t_{\eta}^{\ell}: \eta \in \hat{S}^{\ell}\right\rangle, n_{\mathrm{dn}}^{\ell}, n_{\mathrm{up}}^{\ell}, \bar{r}^{\ell}\right) \in \mathcal{G}($ for $\ell<2), \operatorname{lev}\left(S^{0}\right)=\operatorname{lev}\left(S^{1}\right)$,

- $\left\langle t_{\eta}: \eta \in \hat{S}\right\rangle \in \mathrm{FC}(K, \Sigma),\left\langle t_{\eta}: \eta \in \hat{S}\right\rangle \leq\left\langle t_{\eta}^{\ell}: \eta \in \hat{S}^{\ell}\right\rangle$ (for $\ell<2$ ),

- $\operatorname{lev}(S)<n_{\mathrm{dn}}^{0}, n_{\mathrm{up}}^{0}<n_{\mathrm{dn}}^{1}, F\left(n_{\mathrm{up}}^{1}\right)<\operatorname{lev}\left(S^{1}\right)$,

then: there is $\left(\left\langle t_{\eta}^{*}: \eta \in \hat{S}^{*}\right\rangle, n_{\mathrm{dn}}^{*}, n_{\mathrm{up}}^{*}, \bar{r}^{*}\right) \in \mathcal{G}$ such that

- $n_{\mathrm{dn}}^{*}=n_{\mathrm{dn}}^{0}, n_{\mathrm{up}}^{*}=n_{\mathrm{up}}^{1}, \bar{r}^{*}=\bar{r}^{0} \cup \bar{r}^{1}, \operatorname{lev}\left(S^{*}\right)=\operatorname{lev}\left(S^{0}\right)=\operatorname{lev}\left(S^{1}\right)$,

- $S \subseteq S^{*}$ and $t_{\eta}^{*}=t_{\eta}$ for $\eta \in \hat{S}$,

- $\left\langle t_{\eta}^{*}: \eta \in \hat{S}^{*}\right\rangle \leq\left\langle t_{\eta}^{\ell}: \eta \in \hat{S}^{\ell}\right\rangle($ for $\ell<2)$. 
Remark 2.3.4. In Definition 2.3.5 below, we may think about the forcing notion $\mathbb{Q}^{\text {tree }}(\mathfrak{p})$ in the following way. We have a criterion for "small trees" provided by $\mathcal{G}$ and $\mathcal{F}$ (these are $(\mathcal{G}, f)$-narrow trees, see 2.3.5 (c)). We try to add a small tree that will almost cover all small trees from the ground model. So, naturally, a condition $p$ consists of a small tree (it is the system $\left\langle t_{\eta}^{p}: \eta \in T^{p}\right\rangle$ ), in which some finite part ("below $N^{p}$ ") is declared to be fixed. Now, when we extend the condition $p$, we cannot change the tree below the level $N^{p}$, but above that level we may increase it. The function $f^{p}$ controls in some sense the "smallness" of the tree $T^{p}$. See more later.

Definition 2.3.5. Let $\mathfrak{p}=(K, \Sigma, \mathcal{F}, \mathcal{G})$ be a universality parameter for $\mathbf{H}$. We define a forcing notion $\mathbb{Q}^{\text {tree }}(\mathfrak{p})$ as follows.

A condition in $\mathbb{Q}^{\text {tree }}(\mathfrak{p})$ is a triple $p=\left(N^{p},\left\langle t_{\eta}^{p}: \eta \in T^{p}\right\rangle, f^{p}\right)$ such that

(a) $\left\langle t_{\eta}^{p}: \eta \in T^{p}\right\rangle \in \mathbb{Q}_{\emptyset}^{\text {tree }}(K, \Sigma), \operatorname{root}\left(T^{p}\right)=\langle\rangle$,

(b) $N^{p} \in \omega, f^{p} \in \mathcal{F}$,

(c) the system $\left\langle t_{\eta}^{p}: \eta \in T^{p}\right\rangle$ is $\left(\mathcal{G}, f^{p}\right)$-narrow, which means:

for infinitely many $n<\omega$, for some $\left(\left\langle t_{\eta}: \eta \in \hat{S}\right\rangle, n_{\mathrm{dn}}, n_{\mathrm{up}}, \bar{r}\right) \in \mathcal{G}$ we have

- $n_{\mathrm{dn}}=n$, and $(\forall i \in \operatorname{dom}(\bar{r}))\left(r_{i} \leq f^{p}(i)\right)$ and

- if $\eta \in T^{p}, \operatorname{lh}(\eta)<\operatorname{lev}(S)$, then $\eta \in S$ and $t_{\eta}^{p} \in \Sigma\left(t_{\eta}\right)$.

The relation $\leq$ on $\mathbb{Q}^{\text {tree }}(\mathfrak{p})$ is given by:

$\left(N^{0},\left\langle t_{\eta}^{0}: \eta \in T^{0}\right\rangle, f^{0}\right) \leq\left(N^{1},\left\langle t_{\eta}^{1}: \eta \in T^{1}\right\rangle, f^{1}\right)$ if and only if

- $N^{0} \leq N^{1},\left\langle t_{\eta}^{0}: \eta \in T^{0}\right\rangle \geq\left\langle t_{\eta}^{1}: \eta \in T^{1}\right\rangle$ (in $\mathbb{Q}_{\emptyset}^{\text {tree }}(K, \Sigma)$ ), and

- if $\eta \in T^{1}, \operatorname{lh}(\eta)<N^{0}$ then $\eta \in T^{0}$ and $t_{\eta}^{0}=t_{\eta}^{1}$, and

- $\left(\forall^{\infty} i \in \omega\right)\left(f^{0}(i) \leq f^{1}(i)\right)$.

Proposition 2.3.6. If $\mathfrak{p}=(K, \Sigma, \mathcal{F}, \mathcal{G})$ is a universality parameter, then $\mathbb{Q}^{\text {tree }}(\mathfrak{p})$ is a $\sigma$-centered forcing notion. If additionally $\mathcal{F}$ is countable and $\leq^{*}$-directed then $\mathbb{Q}^{\text {tree }}(\mathfrak{p})$ is Borel ccc.

Proof. It is easy to check that the relation $\leq$ of $\mathbb{Q}^{\text {tree }}(\mathfrak{p})$ is transitive (so $\mathbb{Q}^{\text {tree }}(\mathfrak{p})$ is a forcing notion). Let us argue that it is $\sigma$-centered when $\mathcal{F}$ is countable (the case of $\leq^{*}$-directed $\mathcal{F}$ can be handled similarly).

For $\left\langle t_{\eta}: \eta \in S\right\rangle \in \mathrm{FC}(K, \Sigma), f \in \mathcal{F}$ let

$$
\begin{aligned}
Q_{f}^{\left\langle t_{\eta}: \eta \in S\right\rangle}=\left\{p \in \mathbb{Q}^{\text {tree }}(\mathfrak{p}):\right. & N^{p}=\operatorname{lev}(S) \text { and } S \subseteq T^{p} \text { and } f^{p}=f \text { and } \\
& \left.\left(\forall \eta \in T^{p}\right)\left(\ln (\eta)<N^{p} \Rightarrow \eta \in S \& t_{\eta}^{p}=t_{\eta}\right)\right\} .
\end{aligned}
$$

Claim 2.3.6.1. Each $Q_{f}^{\left\langle t_{\eta}: \eta \in S\right\rangle}$ is a directed subset of $\mathbb{Q}^{\text {tree }}(\mathfrak{p})$.

Proof of the claim. Let $\left(N^{\ell},\left\langle t_{\eta}^{\ell}: \eta \in T^{\ell}\right\rangle, f^{\ell}\right) \in Q_{f}^{\left\langle t_{\eta}: \eta \in S\right\rangle}$ (for $\ell<2$ ). (Thus $\left.N^{\ell}=\operatorname{lev}(S), f^{\ell}=f.\right)$

Let $F^{\mathcal{G}} \in \omega^{\omega}$ be the increasing function given by 2.3.3 $(\varepsilon)$. Pick a sequence

$$
\operatorname{lev}(S)<n_{\mathrm{dn}}^{0,0}<n_{\mathrm{up}}^{0,0}<n_{\mathrm{dn}}^{1,0}<n_{\mathrm{up}}^{1,0}<\ldots<n_{\mathrm{dn}}^{0, k}<n_{\mathrm{up}}^{0, k}<n_{\mathrm{dn}}^{1, k}<n_{\mathrm{up}}^{1, k}<\ldots
$$

such that $F^{\mathcal{G}}\left(n_{\mathrm{up}}^{1, k}\right)+1<n_{\mathrm{dn}}^{0, k+1}$ and (for $\ell<2$ and $k \in \omega$ )

$$
\left(\left\langle t_{\eta}^{\ell}: \eta \in T^{\ell} \& \operatorname{lh}(\eta)<n_{\mathrm{up}}^{\ell, k}\right\rangle, n_{\mathrm{dn}}^{\ell, k}, n_{\mathrm{up}}^{\ell, k}, f\left\lceil\left[n_{\mathrm{dn}}^{\ell, k}, n_{\mathrm{up}}^{\ell, k}\right]\right) \in \mathcal{G}\right.
$$


(possible by the definition of the forcing $\mathbb{Q}^{\text {tree }}(\mathfrak{p})$ and $2.3 .3(\delta)$ ). Now build inductively a system $\left\langle t_{\eta}^{*}: \eta \in T^{*}\right\rangle \in \mathbb{Q}_{\emptyset}^{\text {tree }}(K, \Sigma)$ as follows.

We declare that $\operatorname{root}\left(T^{*}\right)=\operatorname{root}(S)=\langle\rangle$, and if $\eta \in T^{*}, \operatorname{lh}(\eta)<\operatorname{lev}(S)$, then $t_{\eta}^{*}=t_{\eta}$ and $\operatorname{succ}_{T^{*}}(\eta)=\operatorname{pos}\left(t_{\eta}^{*}\right)$.

Suppose we have defined $T^{*}$ up to the level $n_{\mathrm{dn}}^{0, k}-1$, so we know $t_{\eta}^{*}$ for $\operatorname{lh}(\eta)<$ $n_{\mathrm{dn}}^{0, k}-1$. Let $S_{k}$ be the tree of height $n_{\mathrm{dn}}^{0, k}-1$ built from these $t_{\eta}^{*}$ (so it is the respective "initial part" of our future $T^{*}$ ), and assume that $\left\langle t_{\eta}^{*}: \eta \in \hat{S}_{k}\right\rangle \leq\left\langle t_{\eta}^{\ell}: \eta \in T^{\ell}\right\rangle$ (for $\ell=0,1)$. Apply 2.3.3 $(\varepsilon)$ to get $\left\langle t_{\eta}^{*}: \eta \in \hat{S}_{k+1}\right\rangle$ such that

$$
\left(\left\langle t_{\eta}^{*}: \eta \in \hat{S}_{k+1}\right\rangle, n_{\mathrm{dn}}^{0, k}, n_{\mathrm{up}}^{1, k}, f \uparrow\left[n_{\mathrm{dn}}^{0, k}, n_{\mathrm{up}}^{1, k}\right]\right) \in \mathcal{G},
$$

and $S_{k} \subseteq S_{k+1}, \operatorname{lev}\left(S_{k+1}\right)=n_{\mathrm{dn}}^{0, k+1}-1$ and $\left\langle t_{\eta}^{*}: \eta \in \hat{S}_{k+1}\right\rangle \leq\left\langle t_{\eta}^{\ell}: \eta \in T^{\ell}\right\rangle$ (for $\ell<2$ ). We declare that $T^{*}$ up to the level $n_{\mathrm{dn}}^{0, k+1}-1$ is $S_{k+1}$ (and the respective $t_{\eta}^{*}$ are as chosen above).

Plainly, $\left(\operatorname{lev}(S),\left\langle t_{\eta}^{*}: \eta \in T^{*}\right\rangle, f\right) \in Q_{f}^{\left\langle t_{\eta}: \eta \in S\right\rangle}$ is a condition stronger than both $\left(N^{0},\left\langle t_{\eta}^{0}: \eta \in T^{0}\right\rangle, f^{0}\right)$ and $\left(N^{1},\left\langle t_{\eta}^{1}: \eta \in T^{1}\right\rangle, f^{1}\right)$.

The rest should be clear.

\subsection{Examples.}

Example 2.4.1. Let $\mathbf{H}(i)=\omega$. Suppose that $\mathbf{D}, \mathbf{B}, \mathbf{S}$ are functions such that

- $\operatorname{dom}(\mathbf{D}) \subseteq \omega^{<\omega}, \mathbf{D}(\eta)$ is a non-principal ultrafilter on $\omega($ for $\eta \in \operatorname{dom}(\mathbf{D})$ ),

- $\operatorname{dom}(\mathbf{S})=\operatorname{dom}(\mathbf{B})=\omega<\omega \backslash \mathbf{D}(\eta)$ and for each $\eta \in \operatorname{dom}(\mathbf{B})$ :

$$
2 \leq \mathbf{B}(\eta) \in \omega \cup\{\omega\}, \quad \mathbf{S}(\eta)=\left\langle s_{k}^{\eta}: k \in \mathbf{B}(\eta)\right\rangle \subseteq(0,1), \text { and } \sum_{k \in \mathbf{B}(\eta)} s_{k}^{\eta}=1 .
$$

We build a ccc-complete (see 2.1.3) mixing triple $\left(K_{2.4 .1}, 22.42, \mathbf{F} 2.4 .1\right)$ for $\mathbf{H}$ (for the parameters $\mathbf{B}, \mathbf{D}, \mathbf{S})$.

Construction. Let $K$ 2.4.1 consist of all tree creatures $t \in \operatorname{LTCR}[\mathbf{H}]$ such that

- $\operatorname{dis}[t]=\left(n_{t}, \eta_{t}, A_{t}\right)$ for some $n_{t} \in \omega, \eta_{t} \in \prod_{i<n_{t}} \mathbf{H}(i)$ and $A_{t} \subseteq \omega$ such that

$A_{t} \in \mathbf{D}\left(\eta_{t}\right) \quad$ if $\eta_{t} \in \operatorname{dom}(\mathbf{D}), \quad$ and $\quad \emptyset \neq A_{t} \subseteq \mathbf{B}\left(\eta_{t}\right) \quad$ if $\eta_{t} \in \operatorname{dom}(\mathbf{B})$,

- $\operatorname{nor}[t]=n_{t}$,

- $\operatorname{val}[t]=\left\{\left\langle\eta_{t}, \nu\right\rangle: \eta_{t} \triangleleft \nu \in \prod_{i \leq n_{t}} \mathbf{H}(i) \& \nu\left(n_{t}\right) \in A_{t}\right\}$.

The operation 22.4 is natural:

$$
\Sigma \text { 2.4.1 }(t)=\left\{s \in K \text { 2.4.1 }: n_{s}=n_{t} \& \eta_{s}=\eta_{t} \& A_{s} \subseteq A_{t}\right\} .
$$

For $\eta \in \prod_{i<n} \mathbf{H}(i)$ let $t_{\eta}^{*} \in K$ 2.4.1 $\cap \operatorname{LTCR}_{\eta}[\mathbf{H}]$ be such that

$$
A_{t_{\eta}^{*}}= \begin{cases}\omega & \text { if } \eta \in \operatorname{dom}(\mathbf{D}) \\ \mathbf{B}(\eta) & \text { if } \eta \in \operatorname{dom}(\mathbf{B})\end{cases}
$$

and for $\left\langle r_{\nu}: \nu \in \operatorname{pos}\left(t_{\eta}^{*}\right)\right\rangle \in[0,1]^{\operatorname{pos}\left(t_{\eta}^{*}\right)}$ let

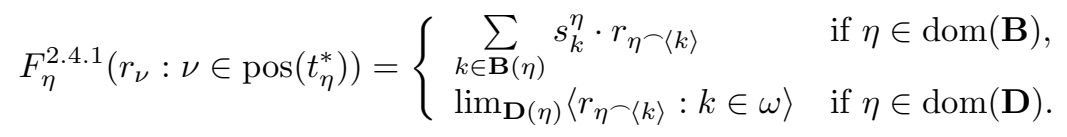


Let $\mathbf{F} 2.4 .1=\left\langle F\right.$.2.1.1 $\left.: \eta \in \bigcup_{n \in \omega} \prod_{i<n} \mathbf{H}(i)\right\rangle$. Check that $(K 2.4 .1, \Sigma 2.4 .1, \mathbf{F} 2.4 .1)$ is a ccc-complete mixing triple for $\mathbf{H}$.

Conclusion 2.4.2. Let $\mathbf{H}, \mathbf{D}, \mathbf{B}$ and $\mathbf{S}$ be as in 2.4.1. Then the forcing notion $\mathbb{Q}_{\emptyset}^{\mathrm{mt}}(K 2.4 .1,22.4 .1, \mathbf{F} 2.4 .1)$ (for the parameters $\left.\mathbf{D}, \mathbf{B}, \mathbf{S}\right)$ is ccc (and non-trivial).

Remark 2.4.3. If $\operatorname{dom}(\mathbf{D})=\omega^{<\omega}$ then $\mathbb{Q}_{\emptyset}^{\mathrm{mt}}(K$ 2.4.1 $, \Sigma 2.4 .1, \mathbf{F} 2.4 .1)$ is the "Laver with ultrafilters" forcing notion.

If $\operatorname{dom}(\mathbf{D})=\emptyset$ and $\mathbf{B}(\eta)$ is finite (for each $\eta$ ) then $\mathbb{Q}_{\emptyset}^{\mathrm{mt}}(K \sqrt{2.4 .1}, 2 \sqrt{2.4 .1}, \mathbf{F} 2.4 .1)$ is the random real forcing (with weights determined by $\mathbf{S}$ in an obvious way).

Between these two extremes we have cases of "mixtures of random with ultrafilters" and our next observation applies to most of them. It could be formulated with a larger generality (e.g. regarding $\operatorname{dom}(\mathbf{D})$ ), what should be clear after reading the proof.

Proposition 2.4.4. Let $\mathbf{H}(n)=\omega$ for $n \in \omega$, and let $X \subseteq \omega$ be an infinite coinfinite set. Suppose that

(a) $\mathbf{D}$ is a function such that $\operatorname{dom}(\mathbf{D})=\left\{\eta \in \omega^{<\omega}: \operatorname{lh}(\eta) \in X\right\}$ and $\mathbf{D}(\eta)$ is a non-principal Ramsey ultrafilter on $\omega($ for $\eta \in \operatorname{dom}(\mathbf{D}))$,

(b) $\bar{n}=\left\langle n_{\ell}: \ell \in \omega \backslash X\right\rangle$ is a sequence of integers, $n_{\ell} \geq 1$,

(c) $\mathbf{B}, \mathbf{S}$ are functions such that $\operatorname{dom}(\mathbf{B})=\operatorname{dom}(\mathbf{S})=\left\{\eta \in \omega^{<\omega}: \operatorname{lh}(\eta) \notin X\right\}$, $\mathbf{B}(\eta)=n_{\operatorname{lh}(\eta)}$ and $\mathbf{S}(\eta)=\left\langle\frac{1}{n_{\operatorname{lh}(\eta)}}: k<n_{\operatorname{lh}(\eta)}\right\rangle$.

Let $(K 2.4 .1, \Sigma 2.4 .1, \mathbf{F} 2.4 .1)$ be the mixing triple built for the parameters $\mathbf{D}, \mathbf{B}, \mathbf{S}$ as in 2.4.1, and let $f \in \omega^{\omega}$ be such that $f(n) \geq 2$ (for $\left.n \in \omega\right)$.

Then for every $\mathbb{Q}_{\emptyset}^{\mathrm{mt}}(K 2.4 .1, \Sigma 2.4 .1, \mathbf{F} 2.4 .1)$ - name $\dot{\tau}$ for a real in $\prod_{i \in \omega} f(i)$, there are an increasing sequence $\left\langle m_{j}: j \in \omega\right\rangle \subseteq \omega$ and a function $g \in \prod_{i \in \omega} f(i)$ such that

$$
\left.\Vdash_{\mathbb{Q}_{\emptyset}^{\mathrm{mt}}(K \text { 2.4.1 }}, 2 \text { 2.4.1 }, \mathbf{F}_{2.4 .1}\right) \text { “ }\left(\forall^{\infty} j \in \omega\right)\left(\dot{\tau} \uparrow\left[m_{j}, m_{j+1}\right) \neq g \uparrow\left[m_{j}, m_{j+1}\right)\right) " .
$$

Hence, in particular, forcing with $\mathbb{Q}_{\emptyset}^{\mathrm{mt}}(K \sqrt[2.4 .1]{, 2}, 2.4 .1, \mathbf{F} 2.4 .1)$ does not add Cohen reals (but it clearly adds a dominating real).

Proof. For notational convenience, let $(K 2.4 .1,22.4 .1, \mathbf{F} 2.4 .1)=(K, \Sigma, \mathbf{F})$.

Note that we may assume that $f(n)>2^{2^{n+2}}$ (as we may work with the mapping $n \mapsto f \uparrow\left[k_{n}, k_{n+1}\right)$ for some increasing $\left\langle k_{n}: n<\omega\right\rangle$ instead). Let $X=\left\{x_{m}\right.$ : $m<\omega\}$ be the increasing enumeration, and let $m_{k}$ be defined by: $m_{0}=0$ and $m_{k+1}=m_{k}+2^{k} \cdot\left(1+\prod_{\ell \in x_{k} \backslash X} n_{\ell}\right)^{k}$ (for $\left.k \in \omega\right)$. [Here we keep the convention that if $x_{k} \backslash X=\emptyset$, then $\prod_{\ell \in x_{k} \backslash X} n_{\ell}=1$; or just assume that $x_{0}>0$.]

Let $\dot{\tau}$ be a $\mathbb{Q}_{\emptyset}^{\mathrm{mt}}(K, \Sigma, \mathbf{F})$-name for an element of $\prod_{i \in \omega} f(i)$, and let $p$ be a condition in $\mathbb{Q}_{\emptyset}^{\mathrm{mt}}(K, \Sigma, \mathbf{F})$. We may assume that $\operatorname{lh}(\operatorname{root}(p)) \in X$, and just for simplicity let $\operatorname{lh}(\operatorname{root}(p))=x_{0}$.

We define inductively a tree $T \subseteq T^{p}$, mappings $Y: T \cap \bigcup_{i<\omega} \omega^{x_{i}+1} \longrightarrow[\omega]^{\omega}$, $\pi: T \cap \bigcup_{i<\omega} \omega^{x_{i}+1} \longrightarrow \omega$, a function $g \in \prod_{i \in \omega} f(i)$, and a system $\left\langle q_{\eta}: \eta \in T \cap\right.$ $\left.\bigcup_{i<\omega} \omega^{x_{i}+1}\right\rangle$ of conditions from $\mathbb{Q}_{\emptyset}^{\mathrm{mt}}(K, \Sigma, \mathbf{F})$. 
We declare $\operatorname{root}(T)=\operatorname{root}(p)$. Using 2.1.11 and 2.1.9 we choose an increasing sequence of conditions $\left\langle q_{\operatorname{root}(T)}^{k}: k<\omega\right\rangle$ and values for $g\left(m_{k}\right)<f\left(m_{k}\right)$ (thus defining $\left.g \uparrow\left\{m_{k}: k \in \omega\right\}\right)$ such that

- $q_{\operatorname{root}(T)}^{0} \geq p, \operatorname{root}\left(q_{\operatorname{root}(T)}^{k}\right)=\operatorname{root}(T)$ and $\mu^{\mathbf{F}}\left(q_{\operatorname{root}(T)}^{k}\right)>\frac{3}{4} \mu^{\mathbf{F}}(p)$,

- $q_{\text {root }(p)}^{k} \Vdash \dot{\tau}\left(m_{k}\right) \neq g\left(m_{k}\right)$.

Since $\mathbf{D}(\operatorname{root}(T))$ is a Ramsey ultrafilter we may choose a set $\left\{a_{k}: k<\omega\right\} \in$ $\mathbf{D}(\operatorname{root}(T))$ (the increasing enumeration) such that

$$
\operatorname{root}(T) \smile\left\langle a_{k}\right\rangle \in T^{q_{\operatorname{root}(T)}^{k}} \quad \text { and } \quad \mu^{\mathbf{F}}\left(\left(q_{\operatorname{root}(T)}^{k}\right)^{\left[\operatorname{root}(T) \smile\left\langle a_{k}\right\rangle\right]}\right)>\frac{3}{4} \mu^{\mathbf{F}}(p) .
$$

We declare that $\operatorname{root}(T) \frown a_{k} \in T$ for all $k<\omega$ and we let

$$
\pi\left(\operatorname{root}(T) \prec\left\langle a_{k}\right\rangle\right)=k, \quad q_{\operatorname{root}(T)}\left\langle\left\langle a_{k}\right\rangle=\left(q_{\operatorname{root}(T)}^{k}\right)^{\left[\operatorname{root}(T) \prec\left\langle a_{k}\right\rangle\right] .} .\right.
$$

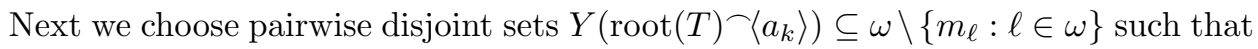
$m_{k+1} \cap Y\left(\operatorname{root}(T) \prec\left\langle a_{k}\right\rangle\right)=\emptyset$ and

$$
(\forall \ell<\omega)\left(\left|Y\left(\operatorname{root}(T) \frown\left\langle a_{k}\right\rangle\right) \cap\left(m_{\ell+k+1}, m_{\ell+k+2}\right)\right|=1\right) .
$$

Suppose now that we have already defined $T \cap \omega^{x_{i}+1}, i<\omega$, together with $Y(\eta) \subseteq \omega \backslash\left\{m_{k}: k \in \omega\right\}, \pi(\eta) \in \omega, q_{\eta} \in \mathbb{Q}_{\emptyset}^{\mathrm{mt}}(K, \Sigma, \mathbf{F})$ (for $\eta \in T \cap \omega^{x_{j}+1}, j \leq i$ ) and

$$
g_{i} \stackrel{\text { def }}{=} g \uparrow\left(\left\{m_{k}: k<\omega\right\} \cup \bigcup\left\{Y(\eta): \eta \in T \cap \omega^{x_{j}+1}, j<i\right\}\right)
$$

so that the following conditions are satisfied:

$(\alpha)$ if $\nu \triangleleft \eta$, and $\pi(\nu), \pi(\eta)$ are defined, then $\pi(\nu)<\pi(\eta)$,

( $\beta)$ if $\nu \in T \cap \omega^{x_{j}}, j \leq i$, then there are no repetitions in the sequence $\langle\pi(\eta)$ : $\left.\nu \triangleleft \eta \in T \cap \omega^{x_{j}+1}\right\rangle$,

$(\gamma)$ if $\eta \in T \cap \omega^{x_{j}+1}, j \leq i$, then

$$
Y_{\eta} \cap m_{\pi(\eta)+1}=\emptyset \quad \text { and } \quad(\forall \ell<\omega)\left(\left|Y_{\eta} \cap\left[m_{\pi(\eta)+\ell+1}, m_{\pi(\eta)+\ell+2}\right)\right|=1\right),
$$

$(\delta)$ the (defined) $Y(\eta)$ 's are pairwise disjoint,

( $\varepsilon$ ) for each $\eta \in T \cap \omega^{x_{i}+1}$ we have: $\operatorname{root}\left(q_{\eta}\right)=\eta$ and

$$
q_{\eta} \Vdash(\forall k \leq \pi(\eta))\left(g_{i} \uparrow\left[m_{k}, m_{k+1}\right) \nsubseteq \dot{\tau}\right),
$$

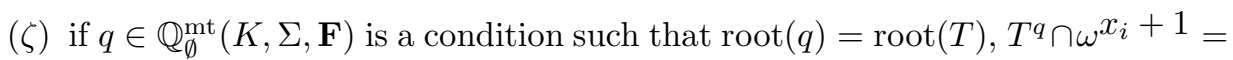
$T \cap \omega^{x_{i}+1}$ and $q^{[\eta]}=q_{\eta}$ for all $\eta \in \omega^{x_{i}+1}$, then $\mu^{\mathbf{F}}(q) \geq\left(\frac{1}{2}+\frac{1}{2^{i+2}}\right) \mu^{\mathbf{F}}(p)$. [Check that these conditions are satisfied at the first stage of the construction.] Note that it follows from clauses $(\alpha)$ and $(\beta)$ that for each $k$ we have

$$
\left|\left\{\eta \in T \cap \omega \leq x_{i}+1: \pi(\eta) \leq k\right\}\right| \leq 2^{k}\left(1+\prod_{\ell \in x_{i} \backslash X} n_{\ell}\right)^{k},
$$

and hence (by clause $(\gamma)$ )

$$
\left|\left[m_{k+1}, m_{k+2}\right) \cap \bigcup\left\{Y(\eta): \eta \in T \cap \omega \leq x_{i}+1\right\}\right| \leq 2^{k}\left(1+\prod_{\ell \in x_{i} \backslash X} n_{\ell}\right)^{k} .
$$

Fix $\eta \in T \cap \omega^{x_{i}+1}$ (and note that $\pi(\eta) \geq i$ ). Choose an increasing sequence $\left\langle q_{\eta}^{k}: \pi(\eta)<k<\omega\right\rangle$ of conditions in $\mathbb{Q}_{\emptyset}^{\mathrm{mt}}(K, \bar{\Sigma}, \mathbf{F})$ and values for $g \mid Y_{\eta}$ such that

- $q_{\eta}^{k} \geq q_{\eta}, \operatorname{root}\left(q_{\eta}^{k}\right)=\eta$ and $\mu^{\mathbf{F}}\left(q_{\eta}^{k}\right)>\left(1-2^{-(i+4)}\right) \mu^{\mathbf{F}}\left(q_{\eta}\right)$,

- if $j \in Y_{\eta} \cap\left[m_{k}, m_{k+1}\right), \pi(\eta)<k<\omega$, then $q_{\eta}^{k} \Vdash \dot{\tau}(j) \neq g(j)$, 
- the sequence $\left\langle T^{q_{\eta}^{k}} \cap \omega^{x_{i+1}}: \pi(\eta)<k<\omega\right\rangle$ is constant (and let $\left\{\nu_{\ell}: \ell<\ell^{*}\right\}$ be the enumeration of $T^{q_{2}^{k}} \cap \omega^{x_{i}+1}$; necessarily $\left.\ell^{*} \leq \prod_{k=x_{i}+1}^{x_{i+1}-1} n_{k}\right)$,

- $r_{\ell} \stackrel{\text { def }}{=} \lim _{k \rightarrow \infty} \mu^{\mathbf{F}}\left(\left(q_{\eta}^{k}\right)^{\left[\nu_{\ell}\right]}\right)>0$ for each $\ell<\ell^{*}$ (note that the limit exists as the sequence is non-increasing).

[Why possible? Use 2.1.11 and 2.1.9, remember our additional assumption on $f$.] Choose $a_{k}^{\ell} \in \omega\left(\right.$ for $\ell<\ell^{*}$ and $k>\pi(\eta)$ ) so that:

- $\left\{a_{k}^{\ell}: \pi(\eta)<k<\omega\right\} \in \mathbf{D}\left(\nu_{\ell}\right)$,

- $\nu_{\ell} \prec\left\langle a_{k}^{\ell}\right\rangle \in T^{q_{\eta}^{k}}$ and $\mu^{\mathbf{F}}\left(\left(q_{\eta}^{k}\right)^{\left[\eta-\left\langle a_{k}^{\ell}\right\rangle\right]}\right)>\left(1-2^{-(i+4)}\right) r_{\ell}$

(remember that each $\mathbf{D}\left(\nu_{\ell}\right)$ is a Ramsey ultrafilter). Now we declare that $\nu_{\ell} \frown a_{k}^{\ell} \in T$ for all $\ell<\ell^{*}$ and $\pi(\eta)<k<\omega$ and we let

$$
\pi\left(\nu_{\ell} \prec\left\langle a_{k}^{\ell}\right\rangle\right)=k, \quad q_{\nu_{\ell} \prec\left\langle a_{k}^{\ell}\right\rangle}=\left(q_{\eta}^{k}\right)^{\left[\nu_{\ell} \prec\left\langle a_{k}^{\ell}\right\rangle\right]} .
$$

This finishes the definitions of $T \cap \omega \leq x_{i+1}+1$ and of $\pi(\nu), q_{\nu}$ for $\nu \in T \cap \omega^{x_{i+1}+1}$. It should be clear that (the respective variants of) clauses $(\alpha),(\beta),(\varepsilon)$ and $(\zeta)$ are satisfied. Using $\left(\otimes_{k}\right)$ we may easily choose sets $Y(\nu)\left(\right.$ for $\left.\nu \in T \cap \omega^{x_{i+1}+1}\right)$ so that the demands $(\gamma),(\delta)$ hold. The construction is finished.

The tree $T$ is perfect and it determines a condition $q \in \mathbb{Q}_{\emptyset}^{\text {tree }}(K, \Sigma)$.

Claim 2.4.4.1. $\mu^{\mathbf{F}}(q)>0$ (so $\left.q \in \mathbb{Q}_{\emptyset}^{\mathrm{mt}}(K, \Sigma, \mathbf{F})\right)$.

Proof of the claim. First note that the clause $(\zeta)$ is not enough to show this, as there are fronts in $T^{q}$ which are not included in any $T^{q} \cap \omega \leq x_{i}+1$. However, we may use the specific way the construction was carried out to build a semi-Fmeasure $\mu: T^{q} \longrightarrow[0,1]$ such that $\mu(\operatorname{root}(q))=\frac{3}{8} \mu^{\mathbf{F}}(p)>0$ (what is enough by 2.1.8). So, if $\eta \in T \cap \omega^{x_{i}+1}, i<\omega$, then we let $\mu(\eta)=\left(1-\frac{1}{2^{i+1}}\right) \mu^{\mathbf{F}}\left(q_{\eta}\right)$; if $\eta \in T$, $x_{i}+1<\operatorname{lh}(\eta) \leq x_{i+1}$ then $\mu(\eta)=F_{\eta}\left(\mu(\nu): \nu \in \operatorname{succ}_{T}(\eta)\right)$. Now check.

Thus $q$ is a condition stronger than $p$ and it forces that $(\forall k \in \omega)\left(\dot{\tau} \uparrow\left[m_{k}, m_{k+1}\right) \neq\right.$ $\left.g \uparrow\left[m_{k}, m_{k+1}\right)\right)$. Since $\mathbb{Q}_{\emptyset}^{\mathrm{mt}}(K, \Sigma, \mathbf{F})$ satisfies the ccc, we may easily finish.

Our next example is a small modification of 1.5.14. In a similar way we may modify other examples from the previous section to produce more strongly linked creating pairs.

Example 2.4.5. Let $\mathbf{H} \in \omega^{\omega}$ be a strictly increasing function such that $\mathbf{H}(0)>2$. We construct a really finitary, strongly ${ }^{+}$linked creating pair $(K 2.4 .5,22.4 .5)$ for $\mathbf{H}$ which is satisfies the demands of 2.2.6 (in particular $(*)$ there).

Construction. The family $K$ 2.4.5 consists of creatures $t \in \mathrm{CR}[\mathbf{H}]$ such that

- $\operatorname{dis}[t]=\left(m_{t}, E_{t}\right)$ such that $m_{t}<\omega$ and $\emptyset \neq E_{t} \subseteq \mathbf{H}\left(m_{t}\right) \backslash\{0\}$,

- $\operatorname{val}[t]=\left\{\langle u, v\rangle \in \prod_{i<m_{t}} \mathbf{H}(i) \times \prod_{i \leq m_{t}} \mathbf{H}(i): u \triangleleft v \& v\left(m_{t}\right) \notin E_{t}\right\}$,

- $\operatorname{nor}[t]=\log _{4}\left(\frac{\mathbf{H}\left(m_{t}\right)}{\left|E_{t}\right|}\right)$.

The operation 22.4 .5 is natural:

$$
\Sigma 2.4 .5(t)=\left\{s \in K \text { 2.4.5: } m_{s}=m_{t} \& E_{t} \subseteq E_{s}\right\} \text {. }
$$

Now check. 
Conclusion 2.4.6. Suppose that $\mathbf{H} \in \omega^{\omega}$ is strictly increasing, $\mathbf{H}(0)>2$ and $(K \sqrt{2.4 .5}, \Sigma 2.4 .5)$ is built as in 2.4.5 for $\mathbf{H}$. Let $D$ be a Ramsey ultrafilter on $\omega$. Then the forcing notion $\mathbb{Q}_{D}^{*}(K \overline{2.4 .5}, 22.42)$ is $\sigma$-centered, adds a Cohen real and adds a dominating real.

Now we turn to universality parameters. As said before, one of the prototypes here is the Universal Meager forcing notion. Let us represent it as $\mathbb{Q}^{\text {tree }}(\mathfrak{p})$ (for a suitable $\mathfrak{p}$ ).

Example 2.4.7. We construct a universality parameter $\mathfrak{p}$ such that $\mathbb{Q}^{\text {tree }}(\mathfrak{p})$ is the Universal Meager forcing notion.

Construction. Let $\mathbf{H}: \omega \longrightarrow \omega \backslash 2$ and let $K$ consists of tree creatures $t$ for $\mathbf{H}$ such that

- $\operatorname{dis}[t]=\left(m_{t}, \eta_{t}, A_{t}\right)$ for some $m_{t}<\omega, \eta_{t} \in \bigcup_{i<m_{t}} \mathbf{H}(i)$ and $\emptyset \neq A_{t} \subseteq \mathbf{H}\left(m_{t}\right)$,

- $\operatorname{nor}[t]=\left|A_{t}\right|$,

- $\operatorname{val}[t]=\left\{\left\langle\eta_{t}, \eta_{t} \prec\langle a\rangle\right\rangle: a \in A_{t}\right\}$.

The operation $\Sigma$ is natural, so $s \in \Sigma(t)$ if and only if $\eta_{s}=\eta_{t}$ and $A_{s} \subseteq A_{t}$. Let $\mathcal{F}=\{f\}, f(i)=0$.

$\mathcal{G}$ consists of quadruples $\left(\left\langle t_{\eta}: \eta \in \hat{S}\right\rangle, n_{\mathrm{dn}}, n_{\mathrm{up}}, \bar{r}\right)$ such that

- $\left\langle t_{\eta}: \eta \in \hat{S}\right\rangle \in \mathrm{FC}(K, \Sigma)$,

- $n_{\mathrm{dn}} \leq n_{\mathrm{up}} \leq \operatorname{lev}(S)$,

- $\bar{r}=\left\langle r_{i}: i \in \operatorname{dom}(\bar{r})\right\rangle, r_{i}<\omega, \operatorname{dom}(\bar{r}) \subseteq\left[n_{\mathrm{dn}}, n_{\mathrm{up}}\right]$,

- if $\eta \in S, \operatorname{lh}(\eta)=n_{\mathrm{dn}}$ then for some $\nu \in S$ we have $\eta \unlhd \nu, \operatorname{lh}(\nu)<n_{\mathrm{up}}$ and $\operatorname{nor}\left[t_{\nu}\right]<\mathbf{H}(\operatorname{lh}(\nu))$.

Easily $\mathfrak{p}=(K, \Sigma, \mathcal{F}, \mathcal{G})$ is a universality parameter and $\mathbb{Q}^{\text {tree }}(\mathfrak{p})$ is the Universal Meager forcing notion,

Remark 2.4.8. Our next example 2.4.9 captures a number of constructions related to the $\mathrm{PP}$-property. Under the assumptions on $(K, \Sigma)$ as there, we may think that we have a way to measure how large splittings are, and this fully determines what are the tree-creatures in $K$ (and what are the norms). The function $\mathbf{F}$ is used to define (possibly totally not related) norms of sets of nodes of the same length. Thus $\mathbf{F}$ may just count how many elements are in $\max (S)$ (in this case the universality parameter given by 2.4.9 is related to the $\mathrm{PP}$-property). Other possibilities for $\mathbf{F}$ include taking the maximum value of nor $\left[t_{\eta}\right]$, or taking the product of all relevant nor $\left[t_{\eta}\right]$ 's.

Example 2.4.9. Assume that $\mathbf{H}: \omega \longrightarrow \omega \backslash 2$ is strictly increasing and a family $\mathcal{F} \subseteq \omega^{\omega}$ is either countable or $\leq^{*}$-directed [note the direction of the inequality]. Let $(K, \Sigma)$ be a local, really finitary, tree-creating pair for $\mathbf{H}$ such that

- for each $\eta \in \prod_{i<n} \mathbf{H}(i), n<\omega$ and a nonempty $A \subseteq \mathbf{H}(n)$ there is a unique $t_{\eta, A} \in \operatorname{LTCR}_{\eta}[\mathbf{H}] \cap K$ with $\operatorname{pos}\left(t_{\eta, A}\right)=\{\eta\langle\langle a\rangle: a \in A\}$, and

- if $|A|=1$, then nor $\left[t_{\eta, A}\right] \leq 1$, and

- if $\emptyset \neq B \subseteq A \subseteq \mathbf{H}(n)$, then $t_{\eta, B} \in \Sigma\left(t_{\eta, A}\right)$ and $\operatorname{nor}\left[t_{\eta, B}\right] \leq \operatorname{nor}\left[t_{\eta, A}\right]$.

Furthermore, let $\mathbf{F}: \mathrm{FC}(K, \Sigma) \longrightarrow \mathbb{R}^{\geq 0}$ be such that

- if $\left\langle t_{\eta}: \eta \in \hat{S}\right\rangle \in \mathrm{FC}(K, \Sigma), \operatorname{nor}\left[t_{\eta}\right] \leq 1$ (for $\left.\eta \in \hat{S}\right)$, then $\mathbf{F}\left(\left\langle t_{\eta}: \eta \in \hat{S}\right\rangle\right) \leq 1$, and 
- if $\left\langle t_{\eta}^{\ell}: \eta \in \hat{S}\right\rangle \in F C(K, \Sigma)$ (for $\left.\ell<2\right)$, and $\left\langle t_{\eta}^{0}: \eta \in \hat{S}\right\rangle \leq\left\langle t_{\eta}^{1}: \eta \in \hat{S}\right\rangle$, then $\mathbf{F}\left(\left\langle t_{\eta}^{1}: \eta \in \hat{S}\right\rangle\right) \leq \mathbf{F}\left(\left\langle t_{\eta}^{0}: \eta \in \hat{S}\right\rangle\right)$.

We construct $\mathcal{G}=\mathcal{G}_{\mathbf{F}}^{K, \Sigma}$ such that $(K, \Sigma, \mathcal{F}, \mathcal{G})$ is a universality parameter.

Construction. For a nonempty set $Y \subseteq \prod_{j<i} \mathbf{H}(j), i<\omega$, we define $\operatorname{NOR}(Y)$ as the value of $\mathbf{F}\left(\left\langle t_{\eta}: \eta \in \hat{S}\right\rangle\right)$ for $S$ such that $\max (S)=Y$ and $\operatorname{root}(S)=\langle\rangle$. (Note that under our assumptions on $(K, \Sigma)$ there is exactly one such $\left\langle t_{\eta}: \eta \in \hat{S}\right\rangle \in \mathrm{FC}(K, \Sigma)$.)

Let $\mathcal{G}$ consists of all quadruples $\left(\left\langle t_{\eta}: \eta \in \hat{S}\right\rangle, n_{\mathrm{dn}}, n_{\mathrm{up}}, \bar{r}\right)$ satisfying the demands of $2.3 .3(\gamma)$ and such that

for some sequence $\left\langle Y_{i}: i \in \operatorname{dom}(\bar{r})\right\rangle$ we have

- $Y_{i} \subseteq \prod_{j<i} \mathbf{H}(j), \operatorname{NOR}\left(Y_{i}\right) \leq r_{i}$,

- $\left(\forall \eta \in S \cap \prod_{j<n_{\mathrm{up}}} \mathbf{H}(j)\right)(\exists i \in \operatorname{dom}(\bar{r}))\left(\eta\left\lceil i \in Y_{i}\right)\right.$.

Now check.

Example 2.4.10. A universality parameter $\mathfrak{p}$ such that $\mathbb{Q}^{\text {tree }}(\mathfrak{p})$ is the "universal closed measure zero" forcing notion.

Construction. Let $\mathbf{H},(K, \Sigma)$ and $\mathcal{F}$ be as defined in the construction for 2.4.7.

Let $\mathcal{G}$ consists of all quadruples $\left(\left\langle t_{\eta}: \eta \in \hat{S}\right\rangle, n_{\mathrm{dn}}, n_{\mathrm{up}}, \bar{r}\right)$ satisfying the demands of $2.3 .3(\gamma)$ and such that

$$
\frac{\left|S \cap \prod_{i<n_{\text {up }}} \mathbf{H}(i)\right|}{\left|\prod_{i<n_{\text {up }}} \mathbf{H}(i)\right|} \leq \sum_{i \in \operatorname{dom}(\bar{r})} \frac{1}{(i+1)^{2}} .
$$

Let $\mathfrak{p}=(K, \Sigma, \mathcal{F}, \mathcal{G})$. Note that the forcing notion $\mathbb{Q}^{\text {tree }}(\mathfrak{p})$ is equivalent to $\mathbb{Q}$ defined as follows.

A condition in $\mathbb{Q}$ is a pair $(N, T)$ such that $N<\omega$ and $T \subseteq \bigcup_{n<\omega} \prod_{i<n} \mathbf{H}(i)$ is a tree such that $[T]$ is a measure zero subset of $\prod_{i<\omega} \mathbf{H}(i)$;

the order of $\mathbb{Q}$ is the natural one: $\left(N_{0}, T_{0}\right) \leq\left(N_{1}, T_{1}\right)$ if and only if $N_{0} \leq N_{1}$, $T_{0} \subseteq T_{1}$ and $T_{1} \cap \prod_{i<N_{0}} \mathbf{H}(i) \subseteq T_{0}$

\section{INTERLUDE: IDEALS}

Here we introduce $\sigma$-ideals determined by forcing notions discussed in this paper. Most of the content of this part is well known and belongs to folklore (some of this material is presented in Judah and Rosłanowski [7], [6]).

3.1. Generic ideals. We will show how a Souslin ccc forcing notion adding one real produces a ccc Borel $\sigma$-ideal on some Polish space. While we could do this in a larger generality (e.g., considering any name for a real, not only the ones of the form specified in 3.1.1 (3) below, compare [22, §4] and [23, §6, §7]), we have decided to use the specific form of the forcing notions we want to deal with and simplify the notation and arguments (loosing slightly on generality, but it will be clear how possible generalizations go). 
Context 3.1.1. $\quad$ 1. $\mathbf{H}: \omega \longrightarrow \mathcal{H}\left(\omega_{1}\right),|\mathbf{H}(n)| \geq 2$ for all $n \in \omega ; \mathcal{T}_{n}=\prod_{i<n} \mathbf{H}(i)$ (for $n \in \omega$ ) and $\mathcal{T}=\bigcup_{n<\omega} \mathcal{T}_{n}$. Let $\mathcal{X}=\prod_{n \in \omega} \mathbf{H}(n)=[\mathcal{T}]$ be equipped with the natural product (Polish) topology.

2. $\mathbb{P}$ is a Souslin ccc forcing notion with a parameter $r \in 2^{\omega}$ (which also encodes $\mathbf{H})$, so we have $\Sigma_{1}^{1}$-formulas $\varphi_{0}(\cdot, r), \varphi_{1}(\cdot, \cdot, r), \varphi_{2}(\cdot, \cdot, r)$ defining $\mathbb{P}, \leq_{\mathbb{P}}$ and $\perp_{\mathbb{P}}$, respectively.

3. $\dot{W}=\left\langle p_{\eta}: \eta \in \mathcal{T}\right\rangle \subseteq \mathbb{P}$ is such that

$(\alpha) p_{\langle\rangle}=\emptyset_{\mathbb{P}}$, and if $\eta \triangleleft \nu \in \mathcal{T}$ then $p_{\eta} \leq p_{\nu}$

$(\beta)\left\langle p_{\eta}\langle a\rangle: a \in \mathbf{H}(\operatorname{lh}(\eta))\right\rangle$ is a maximal antichain above $p_{\eta}$,

$(\gamma)$ for each $p \in \mathbb{P}$ there is $n<\omega$ such that

$$
\mid\left\{\eta \in \mathcal{T}_{n}: p, p_{\eta} \text { are compatible }\right\} \mid \geq 2,
$$

$(\delta)$ if $p, q \in \mathbb{P}$ are incompatible, then there is $\eta \in \mathcal{T}$ such that $p, p_{\eta}$ are compatible but $q, p_{\eta}$ are incompatible.

[We will treat $\dot{W}$ as a $\mathbb{P}$-name for a real in $\mathcal{X}$ such that $p_{\eta} \Vdash \eta \triangleleft \dot{W}$, and so $\Vdash \dot{W} \notin \mathbf{V}$.]

Definition 3.1.2. For $\mathbb{P}, \dot{W}, \mathbf{H}, \mathcal{X}$ as in 3.1.1, let $\mathcal{I}_{\mathbb{P}, \dot{W}}$ be the collection of all Borel subsets $B$ of $\mathcal{X}$ such that

$$
\Vdash_{\mathbb{P}} “ \dot{W} \notin B " .
$$

Proposition 3.1.3. $\quad$ 1. $\mathcal{I}_{\mathbb{P}, \dot{W}}$ is a ccc $\sigma$-ideal of Borel subsets of $\mathcal{X}$.

2. $\mathcal{I}_{\mathbb{P}, \dot{W}}$ contains all singletons.

3. Let $\psi(p, c)$ be the formula

" $p \in \mathbb{P}$ and $c \in 2^{\omega}$ is a Borel code (for a set $\sharp c \subseteq \mathcal{X}$ ) and $p \Vdash \dot{W} \in \sharp c$ ".

(a) If $M$ is a transitive model of $\mathrm{ZFC}^{*}, p, r, c, \dot{W} \in M$, then

$$
\psi(p, c) \quad \Leftrightarrow \quad M \models \psi(p, c) .
$$

(b) There are $a \Sigma_{2}^{1}$-formula $\psi_{0}$ and $a \Pi_{2}^{1}$-formula $\psi_{1}$ (both with the parameter r) such that

$$
\psi(p, c) \equiv \psi_{0}(p, c) \equiv \psi_{1}(p, c)
$$

(i.e., the equivalences are provable in $\mathrm{ZFC}$ ).

4. The formula " $c \in 2^{\omega}$ is a Borel code (for a set $\sharp c \subseteq \mathcal{X}$ ) and $\sharp c \in \mathcal{I}_{\mathbb{P}, \dot{W}}$ " is absolute between transitive models of $\mathrm{ZFC}^{*}$ (containing $\left.r, \dot{W}, c\right)$.

Proof. (1), (2) Straightforward.

(3) See (the proof of) [1, Lemma 3.6.12].

(4) Follows from (3) and the definition of $\mathcal{I}_{\mathbb{P}, \dot{W}}$ (remember that "being a maximal antichain of $\mathbb{P}$ ” is absolute; see [1, 3.6.4]).

Definition 3.1.4. Let $\mathbb{P}, \dot{W}, \mathbf{H}, \mathcal{X}$ be as in 3.1.1.

1. Let $x \in \mathcal{X}$ and $M$ be a transitive model of $\mathrm{ZFC}^{*}, r, \dot{W} \in M$. We say that $x$ is $\mathcal{I}_{\mathbb{P}, \dot{W}}$-generic over $M$, if $x \notin B$ for every Borel set $B$ from $\mathcal{I}_{\mathbb{P}, \dot{W}}$ coded in $M$. 
2. For a condition $p \in \mathbb{P}$ let

$$
S(p)=S_{\dot{W}}(p) \stackrel{\text { def }}{=}\left\{\eta \in \mathcal{T}: p_{\eta}, p \text { are compatible }\right\} .
$$

3. For a maximal antichain $\mathcal{A} \subseteq \mathbb{P}$ let

$$
B_{\mathcal{A}}=B_{\dot{W}, \mathcal{A}} \stackrel{\text { def }}{=}\{x \in \mathcal{X}:(\forall p \in \mathcal{A})(\exists n<\omega)(x\lceil n \notin S(p))\} .
$$

Let $\mathcal{I}_{\mathbb{P}, \dot{W}}^{0}$ be the family of all subsets of $\mathcal{X}$ that can be covered by a set of the form $B_{\mathcal{A}}$ (for a maximal antichain $\mathcal{A} \subseteq \mathbb{P}$ ).

Proposition 3.1.5. (a) For each $p \in \mathbb{P}, S(p)$ is a perfect subtree of $\mathcal{T}$. If $p \leq q$, then $S(q) \subseteq S(p)$. If $p, q \in \mathbb{P}$ and $S(q) \subseteq S(p)$, then $q \Vdash p \in \Gamma_{\mathbb{P}}$.

(b) If $G \subseteq \mathbb{P}$ is a generic filter over $\mathbf{V}$, then $\mathbf{V}[G]=\mathbf{V}\left[\dot{W}^{G}\right]$.

(c) $\mathcal{I}_{\mathbb{P}, \dot{W}}^{0}$ is an ideal of subsets of $\mathcal{X}$; sets $B_{\mathcal{A}}$ (for a maximal antichain $\mathcal{A} \subseteq \mathbb{P}$ ) are $\Pi_{2}^{0}$.

(d) Let $x \in \mathcal{X}$ and $M$ be a transitive model of $\mathrm{ZFC}^{*}, r, \dot{W} \in M$. Then $x$ is $\mathcal{I}_{\mathbb{P}, \dot{W}}$-generic over $M$ if and only if there is a $\mathbb{P}^{M}$-generic filter $G \subseteq \mathbb{P}^{M}$ over $M$ such that $\dot{W}^{G}=x$.

(e) $\mathcal{I}_{\mathbb{P}, \dot{W}}$ is the $\sigma$-ideal generated by $\mathcal{I}_{\mathbb{P}, \dot{W}}^{0}$. Every set from $\mathcal{I}_{\mathbb{P}, \dot{W}}$ can be covered by a $\Sigma_{3}^{0}$ set from $\mathcal{I}_{\mathbb{P}, \dot{W}}$.

Proof. Straightforward (or see $\llbracket 7, \S 2]$ ).

Conclusion 3.1.6. The quotient algebra $\operatorname{Borel}(\mathcal{X}) / \mathcal{I}_{\mathbb{P}, \dot{W}}$ is a ccc complete Boolean algebra. The mapping

$$
\pi: \mathbb{P} \longrightarrow \operatorname{Borel}(\mathcal{X}) / \mathcal{I}_{\mathbb{P}, \dot{W}}: p \mapsto[S(p)]_{\mathcal{I}_{\mathbb{P}, \dot{W}}}
$$

satisfies:

1. $\operatorname{rng}(\pi)$ is a dense subset of the algebra $\operatorname{Borel}(\mathcal{X}) / \mathcal{I}_{\mathbb{P}, \dot{W}}$,

2. $(\forall p, q \in \mathbb{P})(p \perp q \Leftrightarrow \pi(p) \cap \pi(q)=\mathbf{0})$,

3. $(\forall p, q \in \mathbb{P})\left(q \Vdash p \in \Gamma_{\mathbb{P}} \Leftrightarrow \pi(q) \subseteq \pi(p)\right)$.

Consequently, $\mathrm{RO}(\mathbb{P}) \cong \operatorname{Borel}(\mathcal{X}) / \mathcal{I}_{\mathbb{P}, \dot{W}}$. Moreover, $\pi$ maps $\dot{W}$ onto the canonical name for the generic real in $\operatorname{Borel}(\mathcal{X}) / \mathcal{I}_{\mathbb{P}, \dot{W}}$, so for a Borel code $c$ we have $\llbracket \dot{W} \in$ $\sharp c \rrbracket_{\mathrm{RO}(\mathbb{P})}=[\sharp c]_{\mathcal{I}_{\mathbb{P}, \dot{W}}}$.

Remark 3.1.7. It follows from 3.1.6 that we have nice description of names for reals in the extensions via $\mathbb{P}$.

1. If $\dot{\tau}$ is a $\mathbb{P}$-name for an element of $\mathcal{X}$, then there is a Borel function $f: \mathcal{X} \longrightarrow$ $\mathcal{X}$ such that $\Vdash_{\mathbb{P}} f(\dot{W})=\dot{\tau}$.

2. If $\dot{B}$ is a $\mathbb{P}$-name for a Borel subset of $\mathcal{X}$, then there is a Borel set $A \subseteq \mathcal{X} \times \mathcal{X}$ such that $\vdash_{\mathbb{P}} \dot{B}=(A)_{\dot{W}}$, where $(A)_{x}=\{y:(x, y) \in A\}$.

(See [1, Lemma 3.7.1].)

3.2. Universality ideals. For a forcing notion $\mathbb{Q}^{\text {tree }}(\mathfrak{p}$ ) (where $\mathfrak{p}$ is a universality parameter) we may consider the ccc ideal defined as in 3.1.2, however there is another Borel $\sigma$-ideal related to $\mathbb{Q}^{\text {tree }}(\mathfrak{p})$ (justifying the term "universality forcing notion").

Definition 3.2.1. Let $\mathfrak{p}=(K, \Sigma, \mathcal{F}, \mathcal{G})$ be a universality parameter for $\mathbf{H}$. 
1. We say that $\mathfrak{p}$ is suitable whenever:

(a) for every $f \in \mathcal{F}$ and $n<\omega$ there is $N>n$ such that if $\left(\left\langle t_{\eta}: \eta \in \hat{S}\right\rangle, n_{\mathrm{dn}}, n_{\mathrm{up}}, f \uparrow\left[n_{\mathrm{dn}}, n_{\mathrm{up}}\right]\right) \in \mathcal{G}, N \leq n_{\mathrm{dn}}$ and $\eta \in \prod_{i<n} \mathbf{H}(i)$, then $\left(\exists \nu \in \prod_{i<\operatorname{lev}(S)} \mathbf{H}(i)\right)(\eta \triangleleft \nu \& \nu \notin S)$,

(b) for every $f \in \mathcal{F}$ and $n<\omega$ there is $N>n$ such that if $\left\langle t_{\eta}: \eta \in \hat{S}\right\rangle \in \mathrm{FC}(K, \Sigma), \operatorname{lev}(S)=n, \eta \in \prod_{i<N} \mathbf{H}(i)$ and $\eta\lceil n \in S$,

then there is $\left(\left\langle t_{\eta}^{*}: \eta \in \hat{S}^{*}\right\rangle, n_{\mathrm{dn}}, n_{\mathrm{up}}, f \uparrow\left[n_{\mathrm{dn}}, n_{\mathrm{up}}\right]\right) \in \mathcal{G}$ such that $n<$ $n_{\mathrm{dn}} \leq n_{\mathrm{up}}<N, S \subseteq S^{*}$ and $t_{\eta}=t_{\eta}^{*}$ for $\eta \in S$ and $\operatorname{root}(S)=\operatorname{root}\left(S^{*}\right)$ and $\eta \in S^{*}$.

2. $\mathcal{I}_{\mathfrak{p}}^{0}$ is the collection of subsets $A$ of $\prod_{i<\omega} \mathbf{H}(i)$ such that for some $f \in \mathcal{F}$ and

a $(\mathcal{G}, f)$-narrow system $\left\langle t_{\eta}: \eta \in T\right\rangle \in \mathbb{Q}_{\emptyset}^{\text {tree }}(K, \Sigma)$ (see 2.3.5 (c)) we have $A \subseteq[T]$.

Trees $T$ as above will be called $(\mathcal{G}, f)$-narrow.

3. $\mathcal{I}_{\mathfrak{p}}$ is the $\sigma$-ideal of subsets of $\prod_{i<\omega} \mathbf{H}(i)$ generated by $\mathcal{I}_{\mathfrak{p}}^{0}$.

4. $\dot{T}_{\mathfrak{p}}$ is a $\mathbb{Q}^{\text {tree }}(\mathfrak{p})$-name such that

$$
\Vdash_{\mathbb{Q}^{\text {tree }}(\mathfrak{p})} \dot{T}_{\mathfrak{p}}=\bigcup\left\{T^{p} \cap \prod_{i<N^{p}} \mathbf{H}(i): p \in \Gamma_{\mathbb{Q}^{\text {tree }}(\mathfrak{p})}\right\} .
$$

Proposition 3.2.2. Let $\mathfrak{p}=(K, \Sigma, \mathcal{F}, \mathcal{G})$ be a suitable universality parameter for H.

1. Every set in $\mathcal{I}_{\mathfrak{p}}^{0}$ is nowhere dense (in the product topology of $\prod_{i<\omega} \mathbf{H}(i)$ ); all singletons belong to $\mathcal{I}_{\mathfrak{p}}^{0}$.

2. $\mathcal{I}_{\mathfrak{p}}$ is a proper Borel $\sigma$-ideal of subsets of $\prod_{i<\omega} \mathbf{H}(i)$.

3. If $\mathcal{F}$ is $\leq^{*}$-directed then $\mathcal{I}_{\mathfrak{p}}^{0}$ is an ideal.

4. In $\mathbf{V}^{\mathbb{Q}^{\text {tree }}(\mathfrak{p})}, \dot{T}_{\mathfrak{p}}$ is a tree with no maximal branches which is $(\mathcal{G}, h)$-narrow for some function $h$ (possibly $h \notin \mathcal{F}$ ). If $\mathcal{F}$ is a singleton, then $h \in \mathcal{F}$.

5. Suppose that $\left\langle\mathbb{P}_{\alpha}, \dot{\mathbb{Q}}_{\alpha}: \alpha<\delta\right\rangle$ is finite support iteration of ccc forcing notions such that for some increasing sequence $\alpha_{0}<\alpha_{1}<\alpha_{2}<\ldots<\delta$, $\dot{\mathbb{Q}}_{\alpha_{n}}$ is (forced to be) $\mathbb{Q}^{\text {tree }}(\mathfrak{p})$. Let $\dot{T}_{n}$ be the name for the tree $\dot{T}_{\mathfrak{p}}$ added at stage $\alpha_{n}$. Then, in $\mathbf{V}^{\mathbb{P}_{\delta}}$, if $T \in \mathbf{V}$ is $(\mathcal{G}, f)$-narrow for some $f \in \mathcal{F}$ then $T \subseteq \dot{T}_{n}$ for some $n<\omega$.

6. If in 5 above we additionally assume that $\mathcal{F}=\{f\}$ then, in $\mathbf{V}^{\mathbb{P}_{\delta}}$, there is a set from $\mathcal{I}_{\mathfrak{p}}$ which contains all Borel sets from $\mathcal{I}_{\mathfrak{p}}$ coded in $\mathbf{V}$.

3.3. Baire Property. Let us discuss the Baire property related to our ideals and remind how we may build models in which all projective sets of reals are regular in this respect.

Definition 3.3.1. Let $\mathcal{I}$ be a $\sigma$-ideal of subsets of a Polish space $\mathcal{X}$. A set $A \subseteq \mathcal{X}$ is said to have $\mathcal{I}$-Baire property if for some Borel set $B \in \operatorname{Borel}(\mathcal{X})$ the symmetric difference $A \triangle B$ is in $\mathcal{I}$.

Sets with $\mathcal{I}$-Baire property constitute a $\sigma$-field of subsets of $\mathcal{X}$. Clearly, every Borel set has $\mathcal{I}$-Baire property. But even more, using the method of Category Base (see Morgan [13], [14]) we may show that, for a number of $\sigma$-ideals $\mathcal{I}$ on $\mathcal{X}$, 
the family of sets with $\mathcal{I}$-Baire property is closed under Souslin operation $\mathcal{A}$ (so it includes all $\Sigma_{1}^{1}$ subsets of $\mathcal{X}$; this applies to, e.g., all ccc Borel ideals on $\mathcal{X}$ ). However, it may happen that there are $\Sigma_{2}^{1}$ subsets of $\mathcal{X}$ that do not have the $\mathcal{I}$ Baire property. Below we present a tool that can be used to build a model in which all projective subsets of $\mathcal{X}$ have the $\mathcal{I}_{\mathbb{P}, \dot{W}}$-Baire property for some pairs $(\mathbb{P}, \dot{W})$ as in 3.1.1. So let us work in the context of 3.1.1.

Let $\mathbb{B}$ be a complete ccc Boolean algebra. Every $\mathbb{B}$-name $\dot{\tau}$ for a real in $\mathcal{X}$ determines a complete subalgebra $\mathbb{B}_{\dot{\tau}}$ of $\mathbb{B}$. This subalgebra is generated by the family $\left\{\llbracket \eta \triangleleft \dot{\tau} \rrbracket_{\mathbb{B}}: \eta \in \mathcal{T}\right\}$ (as a complete subalgebra). If $\dot{\tau}$ is a name for $\mathcal{I}_{\mathbb{P}, \dot{W}^{-}}$ generic real such that $\llbracket \dot{\tau} \in B \rrbracket_{\mathbb{B}} \neq \mathbf{0}$ for every Borel set $B \in \operatorname{Borel}(\mathcal{X}) \backslash \mathcal{I}_{\mathbb{P}, \dot{W}}$, then $\mathbb{B}_{\dot{\tau}}$ is a copy of the algebra $\mathrm{RO}(\mathbb{P})$ (and the isomorphism maps $\dot{W}$ to the name $\dot{\tau}$ ).

Definition 3.3.2. Let $\mathbb{B}$ be a complete ccc Boolean algebra, and let $\mathbb{B}^{\prime} \lessdot \mathbb{B}$ be its complete subalgebra. We say that $\mathbb{B}$ is $(\mathbb{P}, \dot{W})$-homogeneous over $\mathbb{B}^{\prime}$ if

$(\oplus)_{1}$ for each complete embeddings $\mathbf{e}_{1}, \mathbf{e}_{2}: \mathbb{B}^{\prime} * \mathrm{RO}(\mathbb{P}) \longrightarrow \mathbb{B}$ such that $\mathbf{e}_{1} \uparrow \mathbb{B}^{\prime}=$ $\mathbf{e}_{2} \backslash \mathbb{B}^{\prime}=\mathrm{id}_{\mathbb{B}^{\prime}}$ there is an automorphism $\pi$ of $\mathbb{B}$ such that $\mathbf{e}_{2}=\pi \circ \mathbf{e}_{1}$, and

$(\oplus)_{2}$ if $\mathbf{e}: \mathbb{B}^{\prime} * \mathrm{RO}(\mathbb{P}) \longrightarrow \mathbb{B}$ is a complete embedding, $b \in \mathbb{B}$ and $\pi_{0}$ is an automorphism of the complete subalgebra of $\mathbb{B}$ generated by $\mathbf{e}\left[\mathbb{B}^{\prime} * \mathrm{RO}(\mathbb{P})\right] \cup\{b\}$, $\pi_{0}\left\lceil\mathbf{e}\left[\mathbb{B}^{\prime} * \mathrm{RO}(\mathbb{P})\right]=\mathrm{id}\right.$, then there is an automorphism $\pi$ of $\mathbb{B}$ extending $\pi_{0}$.

Theorem 3.3.3 (Solovay). Assume that $\mathbb{B}$ is a complete ccc Boolean algebra, $\mathbb{B}^{\prime}$ is its complete subalgebra, and $\dot{t}$ is a $\mathbb{B}^{\prime}$-name for a real. Suppose that

1. $\Vdash_{\mathbb{B}}$ " the union of all Borel sets from the ideal $\mathcal{I}_{\mathbb{P}, \dot{W}}$ coded in $\mathbf{V}^{\mathbb{B}^{\prime}}$ belongs to the ideal $\mathcal{I}_{\mathbb{P}, \dot{W}}$ ",

2. $\mathbb{B}$ is $(\mathbb{P}, \dot{W})$-homogeneous over $\mathbb{B}^{\prime}$,

3. if $\dot{\tau}$ is a $\mathbb{B}$-name for a real in $\mathcal{X}, \Vdash_{\mathbb{B}}$ " $\dot{\tau}$ is $\mathcal{I}_{\mathbb{P}, \dot{W}}$-generic over $\mathbf{V}^{\mathbb{B}^{\prime}}$ ", then $\dot{\tau}$ is a $\mathbb{B}^{\prime \prime}$-name for some $\mathbb{B}^{\prime \prime}$ such that $\mathbb{B}^{\prime} \lessdot \mathbb{B}^{\prime \prime} \lessdot \mathbb{B}$ and $\mathbb{B}^{\prime \prime} * \mathrm{RO}(\mathbb{P})$ embeds in $\mathbb{B}$ (by an embedding that is identity on $\mathbb{B}^{\prime \prime}$ ).

Then $\mathbb{B} \Vdash$ " any subset of $\mathcal{X}$ definable with $\dot{t}$ and with (real) parameters from $\mathbf{V}$ has the $\mathcal{I}_{\mathbb{P}, \dot{W}}$-Baire property".

Proof. Suppose that $G \subseteq \mathbb{B}$ is a generic filter over $\mathbf{V}$ and $x \in \mathcal{X} \cap \mathbf{V}[G]$ is $\mathcal{I}_{\mathbb{P}, \dot{W}^{-}}$ generic over $\mathbf{V}\left[G \cap \mathbb{B}^{\prime}\right]$. It follows from the third assumption that there is a $\mathbb{B}$ name $\dot{\tau}$ for $x$ such that $\llbracket \dot{\tau} \in \dot{A} \rrbracket_{\mathbb{B}} \neq \mathbf{0}$ for every $\mathbb{B}^{\prime}$-name $\dot{A}$ for an element of $\operatorname{Borel}(\mathcal{X}) \backslash \mathcal{I}_{\mathbb{P}, \dot{W}}$. Consequently, we find a complete embedding $\mathbf{e}: \mathbb{B}^{\prime} * \operatorname{RO}(\mathbb{P}) \longrightarrow \mathbb{B}$ such that $\mathbf{e} \backslash \mathbb{B}^{\prime}=\operatorname{id}_{\mathbb{B}^{\prime}}$ and $\mathbf{e}(\dot{W})=\dot{\tau}$.

Now continue like in the proof of [6, Theorem 2.3]; see also the original Solovay's article [30].

\section{Sweet And Sour}

4.1. On sweetness. Solovay's Theorem 3.3 .3 shows that to build a model with $\left(\mathcal{I}_{\mathbb{P}, \dot{W}^{-}}\right)$Baire property of projective reals we may construct a suitably homogeneous complete Boolean algebra adding enough generic reals. The main tool for building homogeneous Boolean algebras is amalgamation.

Definition 4.1.1. $\quad$ 1. Suppose $\mathbb{P}, \mathbb{Q}$ are forcing notions such that $\mathbb{P} \Leftarrow \operatorname{RO}(\mathbb{Q})$. Then $(\mathbb{Q}: \mathbb{P})$ is a $\mathbb{P}$-name for a forcing notion which is a suborder of $\mathbb{Q}$,

$$
\begin{aligned}
& p \Vdash_{\mathbb{P}} " q \in(\mathbb{Q}: \mathbb{P}) " \quad \text { if and only if } \\
& \text { every } p^{\prime} \in \mathbb{P} \text { stronger than } p \text { is compatible with } q \text { in } \mathbb{Q} \text {. }
\end{aligned}
$$


2. Suppose that $\mathbb{P}, \mathbb{Q}_{0}, \mathbb{Q}_{1}$ are forcing notions and $f_{\ell}: \mathbb{P} \longrightarrow \mathrm{RO}\left(\mathbb{Q}_{\ell}\right)($ for $\ell<2$ ) are complete embeddings. The amalgamation of $\mathbb{Q}_{0}, \mathbb{Q}_{1}$ over $f_{0}, f_{1}$ is

$$
\begin{aligned}
& \mathbb{Q}_{0} \times_{f_{0}, f_{1}} \mathbb{Q}_{1}= \\
& \left\{\left(q_{1}, q_{2}\right) \in \mathbb{Q}_{0} \times \mathbb{Q}_{1}:(\exists p \in \mathbb{P})\left(p \Vdash “ q_{0} \in\left(\mathbb{Q}_{0}: f_{0}[\mathbb{P}]\right) \& q_{1} \in\left(\mathbb{Q}_{1}: f_{1}[\mathbb{P}]\right) "\right)\right\} \\
& \quad \text { ordered in the natural way (so }\left(q_{0}, q_{1}\right) \leq\left(q_{0}^{\prime}, q_{1}^{\prime}\right) \text { if and only if } q_{0} \leq q_{0}^{\prime} \text { and } \\
& \left.q_{1} \leq q_{1}^{\prime}\right) \text {. }
\end{aligned}
$$

On how the repeated use of amalgamations produces homogeneous Boolean algebras see [6]. The main problem with amalgamating is that quite often it produces forcing notions that collapse $\omega_{1}$ (this effect is related to sourness discussed later). While the ccc might not be preserved in amalgamations, some strong variants of it are.

Definition 4.1.2 (Shelah [20, Def. 7.2]; see Judah and Shelah [9, Def. 2.5] too). A triple $(\mathbb{P}, \mathcal{D}, \bar{E})$ is model of sweetness (on $\mathbb{P}$ ) whenever:

(i) $\mathbb{P}$ is a forcing notion, $\mathcal{D}$ is a dense subset of $\mathbb{P}$,

(ii) $\bar{E}=\left\langle E_{n}: n<\omega\right\rangle$, each $E_{n}$ is an equivalence relation on $\mathcal{D}$ such that $\mathcal{D} / E_{n}$ is countable,

(iii) equivalence classes of each $E_{n}$ are directed, $E_{n+1} \subseteq E_{n}$,

(iv) if $\left\{p_{i}: i \leq \omega\right\} \subseteq \mathcal{D}, p_{i} E_{i} p_{\omega}$ (for $i \in \omega$ ) then

$$
(\forall n \in \omega)\left(\exists q \geq p_{\omega}\right)\left(q E_{n} p_{\omega} \&(\forall i \geq n)\left(p_{i} \leq q\right)\right),
$$

(v) if $p, q \in \mathcal{D}, p \leq q$ and $n \in \omega$ then there is $k \in \omega$ such that

$$
\left(\forall p^{\prime} \in[p]_{E_{k}}\right)\left(\exists q^{\prime} \in[q]_{E_{n}}\right)\left(p^{\prime} \leq q^{\prime}\right) .
$$

If there is a model of sweetness on $\mathbb{P}$, then we say that $\mathbb{P}$ is sweet.

Definition 4.1.3 (Stern [31, Def. 1.2]). Let $\mathbb{P}$ be a forcing notion and $\tau$ be a topology on $\mathbb{P}$. We say that $(\mathbb{P}, \tau)$ is a model of topological sweetness whenever the following conditions are satisfied:

(i) the topology $\tau$ has a countable basis,

(ii) $\emptyset_{\mathbb{P}}$ is an isolated point in $\tau$,

(iii) if a sequence $\left\langle p_{n}: n\langle\omega\rangle \subseteq \mathbb{P}\right.$ is $\tau$-converging to $p \in \mathbb{P}, q \geq p$ and $W$ is a $\tau$-neighbourhood of $q$, then there is a condition $r \in \mathbb{P}$ such that

(a) $r \in W, r \geq q$,

(b) the set $\left\{n \in \omega: p_{n} \leq r\right\}$ is infinite.

A forcing notion $\mathbb{P}$ is topologically sweet, if there is a topology $\tau$ on $\mathbb{P}$ such that $(\mathbb{P}, \tau)$ is a model of topological sweetness.

Definition 4.1.4. 1. Let $\left(\mathbb{P}_{\ell}, \mathcal{D}_{\ell}, \bar{E}_{\ell}\right)$ (for $\left.\ell<2\right)$ be models of sweetness. We say that $\left(\mathbb{P}_{1}, \mathcal{D}_{1}, \bar{E}_{1}\right)$ extends the model $\left(\mathbb{P}_{0}, \mathcal{D}_{0}, \bar{E}_{0}\right)$ if

- $\mathbb{P}_{0} \prec \mathbb{P}_{1}, \mathcal{D}_{0} \subseteq \mathcal{D}_{1}$ and $E_{n}^{0}=E_{n}^{1}\left\lceil\mathcal{D}_{0}\right.$ for each $n \in \omega$,

- if $p \in \mathcal{D}_{0}, n \in \omega$, then $[p]_{E_{n}^{1}} \subseteq \mathcal{D}_{0}$,

- if $p \leq q, p \in \mathcal{D}_{1}, q \in \mathcal{D}_{0}$, then $p \in \mathcal{D}_{0}$.

2. Let $\left(\mathbb{P}_{\ell}, \tau_{\ell}\right)$ (for $\ell<2$ ) be models of topological sweetness. We say that $\left(\mathbb{P}_{1}, \tau_{1}\right)$ extends the model $\left(\mathbb{P}_{0}, \tau_{0}\right)$ if $\mathbb{P}_{0} \lessdot \mathbb{P}_{1}, \mathbb{P}_{0}$ is a $\tau_{1}$-open subset of $\mathbb{P}_{1}$ and $\tau_{1} \uparrow \mathbb{P}_{0}=\tau_{0}$. 
Theorem 4.1.5. 1. (Shelah [20, 7.5]) Suppose that $\left(\mathbb{P}_{\ell}, \mathcal{D}_{\ell}, \bar{E}_{\ell}\right)($ for $\ell<2)$ are models of sweetness, and $f_{\ell}: \mathbb{P} \longrightarrow \mathrm{RO}\left(\mathbb{P}_{\ell}\right)$ (for $\ell<2$ ) are complete embeddings. Then there is a model of sweetness $\left(\mathbb{P}_{0} \times_{f_{0}, f_{1}} \mathbb{P}_{1}, \mathcal{D}^{*}, \bar{E}^{*}\right)$ based on the amalgamation $\mathbb{P}_{0} \times_{f_{0}, f_{1}} \mathbb{P}_{1}$ and extending each $\left(\mathbb{P}_{\ell}, \mathcal{D}_{\ell}, \bar{E}_{\ell}\right)$ for $\ell<2$.

2. (Stern [31, §2.2]) Suppose that $\left(\mathbb{P}_{\ell}, \tau_{\ell}\right)($ for $\ell<2)$ are models of topological sweetness, and $f_{\ell}: \mathbb{P} \longrightarrow \mathrm{RO}\left(\mathbb{P}_{\ell}\right)$ (for $\ell<2$ ) are complete embeddings. Then there is a model of topological sweetness $\left(\mathbb{P}_{0} \times f_{0}, f_{1} \mathbb{P}_{1}, \tau^{*}\right)$ based on the amalgamation $\mathbb{P}_{0} \times_{f_{0}, f_{1}} \mathbb{P}_{1}$ and extending each $\left(\mathbb{P}_{\ell}, \tau_{\ell}\right)$ for $\ell<2$.

Definition 4.1.6. Let $\mathcal{B}$ be a countable basis of a topology on a forcing notion $\mathbb{P}$. We say that $(\mathbb{P}, \mathcal{B})$ is a model of iterable sweetness if

(i) $\mathcal{B}$ is closed under finite intersections,

(ii) each $U \in \mathcal{B}$ is directed and $p \leq q \in U \Rightarrow p \in U$,

(iii) if $\left\langle p_{n}: n \leq \omega\right\rangle \subseteq U$ and the sequence $\left\langle p_{n}: n<\omega\right\rangle$ converges to $p_{\omega}$ (in the topology generated by $\mathcal{B})$, then there is a condition $p \in U$ such that $(\forall n \leq \omega)\left(p_{n} \leq p\right)$.

Proposition 4.1.7. Assume that $(\mathbb{P}, \mathcal{D}, \bar{E})$ is a sweetness model on $\mathbb{P}, \bar{E}=\left\langle E_{n}\right.$ : $n\langle\omega\rangle, \mathcal{D}=\mathbb{P}$. Furthermore, suppose that any two compatible elements of $\mathbb{P}$ have a least upper bound, i.e., if $p_{0}, p_{1} \in \mathbb{P}$ are compatible, then there is $q \geq p_{0}, p_{1}$ such that $(\forall r \in \mathbb{P})\left(r \geq p_{0} \& r \geq p_{1} \Rightarrow r \geq q\right)$. For $\bar{p}=\left\langle p_{\ell}: \ell \leq k\right\rangle \subseteq \mathbb{P}$ and $\bar{n}=\left\langle n_{\ell}: \ell \leq k\right\rangle \subseteq \omega$ let

$$
U(\bar{p}, \bar{n}) \stackrel{\text { def }}{=}\left\{q \in \mathbb{P}:(\forall \ell \leq k)\left(\exists q^{\prime} \in\left[p_{\ell}\right]_{E_{n_{\ell}}}\right)\left(q \leq q^{\prime}\right)\right\},
$$

and let $\mathcal{B}$ be the collection of all sets $U(\bar{p}, \bar{n})$ (for $\bar{p} \subseteq \mathbb{P}, \bar{n} \subseteq \omega, \operatorname{lh}(\bar{p})=\operatorname{lh}(\bar{n})$ ). Then $(\mathbb{P}, \mathcal{B})$ is a model of iterable sweetness.

Proof. Plainly, $\mathcal{B}$ is closed under finite intersections. Since the equivalence classes of each $E_{n}$ are directed and any two compatible members of $\mathbb{P}$ have a least upper bound, we may conclude that the elements of $\mathcal{B}$ are directed and downward closed.

Before we verify the demand 4.1.6(iii), let us first note that if $p \in U(\bar{p}, \bar{n})$, $\bar{p}=\left\langle p_{\ell}: \ell \leq k\right\rangle, \bar{n}=\left\langle n_{\ell}: \ell \leq k\right\rangle$, then for some $N$ we have $U(\langle p\rangle,\langle N\rangle) \subseteq U(\bar{p}, \bar{n})$. [Why? Use 4.1.2(v) to choose $N$ such that

$$
(\forall \ell \leq k)\left(\forall q \in[p]_{E_{N}}\right)\left(\exists q^{\prime} \in\left[p_{\ell}\right]_{E_{n_{\ell}}}\right)\left(q \leq q^{\prime}\right) .
$$

Clearly this $N$ is as required.]

Now suppose that a sequence $\left\langle p_{n}: n<\omega\right\rangle$ converges to $p_{\omega}$ in the topology generated by $\mathcal{B}$, and $p_{\omega}, p_{n} \in U(\bar{q}, \bar{n}) \in \mathcal{B}$ for all $n<\omega$. Take $N$ such that $U\left(\left\langle p_{\omega}\right\rangle,\langle N\rangle\right) \subseteq U(\bar{q}, \bar{n})$. Choose an increasing sequence $\left\langle m_{i}: i<\omega\right\rangle$ such that

$$
(\forall i<\omega)\left(\forall n \geq m_{i}\right)\left(p_{n} \in U\left(\left\langle p_{\omega}\right\rangle,\langle N+1+i\rangle\right)\right) .
$$

Next pick conditions $p_{i}^{*} \in\left[p_{\omega}\right]_{E_{N+1+i}}$ such that

$$
(\forall i<\omega)\left(\forall n \in\left[m_{i}, m_{i+1}\right)\right)\left(p_{n} \leq p_{i}^{*}\right)
$$

(remember that each $\left[p_{\omega}\right]_{E_{N+1+i}}$ is directed). It follows from 4.1.2(iv) that we may find a condition $q^{\prime} \geq p_{\omega}$ such that $q^{\prime} \in\left[p_{\omega}\right]_{E_{N}}$ and $(\forall i<\omega)\left(p_{i}^{*} \leq q^{\prime}\right)$. Then

$$
\left(\forall n \geq m_{0}\right)\left(p_{n} \leq q^{\prime}\right) \quad \text { and } \quad q^{\prime} \in U(\bar{q}, \bar{n}) .
$$

Since $U(\bar{q}, \bar{n})$ is directed and $q^{\prime}, p_{0}, \ldots, p_{m_{0}} \in U(\bar{q}, \bar{n})$, the conditions $q^{\prime}, p_{0}, \ldots, p_{m_{0}}$ have an upper bound in $U(\bar{q}, \bar{n})$ - let $q$ be such an upper bound. 
Lemma 4.1.8. Assume that $(\mathbb{P}, \tau)$ is a model of topological sweetness.

1. If $p, q \in \mathbb{P}, p \leq q$ and $q \in U \in \tau$, then there is an open neighbourhood $V$ of $p$ such that

$$
(\forall r \in V)\left(\exists r^{\prime} \in U\right)\left(r \leq r^{\prime}\right) .
$$

2. If $m \in \omega, p \in U \in \tau$, then there is an open neighbourhood $V$ of $p$ such that any $p_{0}, \ldots, p_{m} \in V$ have a common upper bound in $U$.

Proof. Straightforward.

Theorem 4.1.9. Suppose that $(\mathbb{P}, \tau)$ is a model of topological sweetness and $\dot{\mathcal{B}}, \dot{\mathbb{Q}}$ are $\mathbb{P}$-names such that

$$
\Vdash_{\mathbb{P}} \text { “ }(\dot{\mathbb{Q}}, \dot{\mathcal{B}}) \text { is a model of iterable sweetness". }
$$

Then there is dense subset $\mathbb{R}$ of the iteration $\mathbb{P} * \dot{\mathbb{Q}}$ and a topology $\tau^{*}$ on $\mathbb{R}$ such that $\mathbb{P} \subseteq \mathbb{R}$ and $\left(\mathbb{R}, \tau^{*}\right)$ is a model of topological sweetness extending the model $(\mathbb{P}, \tau)$.

Proof. Let $\dot{V}_{n}$ be $\mathbb{P}$-names such that

$$
\Vdash_{\mathbb{P}} " \dot{V}_{0}=\left\{\emptyset_{\dot{\mathbb{Q}}}\right\} \text { and }\left\{\dot{V}_{n}: n<\omega\right\} \text { enumerates } \dot{\mathcal{B}} \backslash\{\emptyset\} "
$$

(note that $\Vdash$ " $\left(\dot{\mathbb{Q}},\left\{\dot{V}_{n}: n<\omega\right\}\right)$ is a model of iterable sweetness " and also $\Vdash$ " $\emptyset_{\dot{\mathbb{Q}}} \in \dot{V}_{n}$ for all $n$ ", remember 4.1.6(ii)). Let $\mathcal{U}$ be a countable basis of the topology $\tau$ and let

$$
\mathbb{R} \stackrel{\text { def }}{=}\left\{(p, \dot{q}) \in \mathbb{P} * \dot{\mathbb{Q}}: p \neq \emptyset_{\mathbb{P}} \text { and }(\exists n<\omega)\left(p \Vdash \dot{q} \in \dot{V}_{n}\right)\right\} \cup\left\{\left(\emptyset_{\mathbb{P}}, \dot{\emptyset}_{\dot{\mathbb{Q}}}\right)\right\} .
$$

For $U \in \mathcal{U}, \bar{U}=\left\langle U_{0}, \ldots, U_{m-1}\right\rangle \subseteq \mathcal{U}$ and $\bar{n}=\left\langle n_{0}, \ldots, n_{m-1}\right\rangle \subseteq \omega$ and $\bar{k}=$ $\left\langle k_{0}, \ldots, k_{M}\right\rangle \subseteq \omega(m, M<\omega)$ we put

$$
\begin{aligned}
U^{*}(U, \bar{U}, \bar{n}, \bar{k}) \stackrel{\text { def }}{=}\{(p, \dot{q}) \in \mathbb{R}: & p \in U \text { and }(\forall \ell \leq M)\left(p \Vdash \dot{q} \in \dot{V}_{k_{\ell}}\right) \text { and } \\
& \left.(\forall \ell<m)\left(\exists p^{\prime} \in U_{\ell}\right)\left(p \leq p^{\prime} \& p^{\prime} \Vdash \dot{q} \in \dot{V}_{n_{\ell}}\right)\right\} .
\end{aligned}
$$

Let $\mathcal{C}$ be the collection of all sets of the form $U^{*}(U, \bar{U}, \bar{n}, \bar{k}$ ) (for suitable parameters $U, \bar{U}, \bar{n}, \bar{k})$.

Claim 4.1.9.1. $\quad$ 1. The family $\mathcal{C}$ forms a countable basis of a topology on $\mathbb{R}$; we will denote this topology by $\tau^{*} . \emptyset_{\mathbb{R}}$ is an isolated point in $\tau^{*}$.

2. If $(p, \dot{q}),\left(p^{\prime}, \dot{q}^{\prime}\right) \in \mathbb{R},(p, \dot{q}) \leq\left(p^{\prime}, \dot{q}^{\prime}\right)$ and $\left(p^{\prime}, \dot{q}^{\prime}\right) \in U^{*} \in \mathcal{C}$, then there is $V^{*} \in \mathcal{C}$ such that $(p, \dot{q}) \in V^{*}$ and $\left(\forall r \in V^{*}\right)\left(\exists r^{\prime} \in U^{*}\right)\left(r \leq r^{\prime}\right)$.

Proof of the claim. 1) Should be clear.

2) Let $U^{*}=U^{*}\left(U,\left\langle U_{0}, \ldots, U_{m-1}\right\rangle,\left\langle n_{0}, \ldots, n_{m-1}\right\rangle,\left\langle k_{0}, \ldots, k_{M}\right\rangle\right)$ and let $p_{\ell}^{\prime} \in U_{\ell}$ (for $\ell<m$ ) be such that $p^{\prime} \leq p_{\ell}^{\prime}$ and $p_{\ell}^{\prime} \Vdash \dot{q}^{\prime} \in \dot{V}_{n_{\ell}}$. Choose $U_{\ell}^{\prime} \in \mathcal{U}$ such that $p_{\ell}^{\prime} \in U_{\ell}^{\prime} \subseteq U_{\ell}$ and every two members of $U_{\ell}^{\prime}$ have a common upper bound in $U_{\ell}$ (possible by 4.1.8(2)). Next pick $U^{\prime}, U^{\prime \prime} \in \mathcal{U}$ such that $p^{\prime} \in U^{\prime \prime} \subseteq U^{\prime} \subseteq U$ and

- every member of $U^{\prime}$ has an upper bound in each of the sets $U_{0}^{\prime}, \ldots, U_{m-1}^{\prime}$,

- every $M+1$ elements of $U^{\prime \prime}$ have a common upper bound in $U^{\prime}$.

Let $U^{+} \in \mathcal{U}$ be such that $p \in U^{+}$and each element of $U^{+}$has an upper bound in $U^{\prime \prime}$, and let $k$ be such that $p \Vdash \dot{q} \in \dot{V}_{k}$. Put

$$
V^{*}=U^{*}\left(U^{+},\left\langle U_{0}^{\prime}, \ldots, U_{m-1}^{\prime}, U^{\prime \prime}, \ldots, U^{\prime \prime}\right\rangle,\left\langle n_{0}, \ldots, n_{m-1}, k_{0}, \ldots, k_{M}\right\rangle,\langle k\rangle\right) \in \mathcal{C} .
$$

First we show that $(p, \dot{q}) \in V^{*}$. By our choices, $p \in U^{+}$and $p \Vdash \dot{q} \in \dot{V}_{k}$. For $\ell<m$, $p_{\ell}^{\prime} \in U_{\ell}^{\prime}$ is a condition stronger than $p^{\prime} \geq p$ and $p_{\ell}^{\prime} \Vdash \dot{q} \leq \dot{q}^{\prime} \in \dot{V}_{n_{\ell}}$, so $p_{\ell}^{\prime} \Vdash \dot{q} \in \dot{V}_{n_{\ell}}$ 
(remember 4.1.6(ii)). Next, for $i \leq M$, we have $p^{\prime} \in U^{\prime \prime}$ and $p^{\prime} \Vdash \dot{q} \leq \dot{q}^{\prime} \in \dot{V}_{k_{i}}$, so $p^{\prime} \Vdash \dot{q} \in \dot{V}_{k_{i}}$.

Now, suppose that $\left(p^{*}, \dot{q}^{*}\right) \in V^{*}$. Then $p^{*} \in U^{+}$and we have conditions $p_{\ell}^{*} \in U_{\ell}^{\prime}$ (for $\ell<m$ ) and conditions $p_{i}^{* *} \in U^{\prime \prime}$ (for $i \leq M$ ) such that

- $p_{\ell}^{*} \geq p^{*}$ and $p_{i}^{* *} \geq p^{*}$

- $p_{\ell}^{*} \Vdash \dot{q}^{*} \in \dot{V}_{n_{\ell}}$ and $p_{i}^{* *} \Vdash \dot{q}^{*} \in \dot{V}_{k_{i}}$.

Pick a condition $p^{+} \in U^{\prime}$ stronger than all $p_{i}^{* *}$ (for $i \leq M$ ). We claim that $\left(p^{+}, \dot{q}^{*}\right) \in U^{*}$. Clearly $p^{+} \in U$ and $p^{+} \Vdash \dot{q}^{*} \in \dot{V}_{k_{i}}($ for $i \leq M)$. Fix $\ell<m$. By the choice of $U^{\prime}$, we find $p_{\ell}^{+} \in U_{\ell}^{\prime}$ stronger than $p^{+}$. By the choice of $U_{\ell}^{\prime}$, we find a condition $p_{\ell} \in U_{\ell}$ stronger than both $p_{\ell}^{+}$and $p_{\ell}^{*}$. Then $p_{\ell} \Vdash \dot{q}^{*} \in \dot{V}_{n_{\ell}}$ and we are done.

Claim 4.1.9.2. Suppose that a sequence $\left\langle\left(p_{k}, \dot{q}_{k}\right): k<\omega\right\rangle \subseteq \mathbb{R}$ is $\tau^{*}$-converging to $\left(p_{\omega}, \dot{q}_{\omega}\right)$, and $\left(p_{\omega}, \dot{q}_{\omega}\right) \in U^{*}\left(U,\left\langle U_{0}, \ldots, U_{m-1}\right\rangle,\left\langle n_{0}, \ldots, n_{m-1}\right\rangle, \bar{k}\right) \in \mathcal{C}$. Then there are an infinite set $X \subseteq \omega$ and conditions $p_{\ell}^{*} \geq p_{\omega}$ (for $\ell<m$ ) such that for each $\ell<m$ and $k \in X$ :

(i) $p_{\ell}^{*} \in U_{\ell}, p_{\ell}^{*} \geq p_{k}$,

(ii) $p_{\ell}^{*} \Vdash \dot{q}_{k} \in \dot{V}_{n_{\ell}}$.

Proof of the claim. Pick $p_{\omega}^{\ell} \in U_{\ell}, p_{\omega}^{\ell} \geq p_{\omega}$ such that $p_{\omega}^{\ell} \Vdash \dot{q}_{\omega} \in \dot{V}_{n_{\ell}}($ for $\ell<m)$. Fix sequences $\left\langle W_{j}^{\ell}: j<\omega\right\rangle \subseteq \mathcal{U}($ for $\ell<m)$ such that

- $p_{\omega}^{\ell} \in W_{j+1}^{\ell} \subseteq W_{j}^{\ell}$,

- $\left\{W_{j}^{\ell}: j<\omega\right\}$ forms a basis of neighbourhoods of $p_{\omega}^{\ell}$.

Clearly $\left(p_{\omega}, \dot{q}_{\omega}\right) \in U^{*}\left(U,\left\langle W_{j}^{0}, \ldots, W_{j}^{m-1}\right\rangle,\left\langle n_{0}, \ldots, n_{m-1}\right\rangle, \bar{k}\right)$ for every $j<\omega$, so we may pick an increasing sequence $\left\langle k_{j}: j<\omega\right\rangle \subseteq \omega$ such that

$$
(\forall j<\omega)\left(\left(p_{k_{j}}, \dot{q}_{k_{j}}\right) \in U^{*}\left(U,\left\langle W_{j}^{0}, \ldots, W_{j}^{m-1}\right\rangle,\left\langle n_{0}, \ldots, n_{m-1}\right\rangle, \bar{k}\right)\right) .
$$

Let $p_{k_{j}}^{\ell} \in W_{j}^{\ell}$ be such that $p_{k_{j}} \leq p_{k_{j}}^{\ell}, p_{k_{j}}^{\ell} \Vdash \dot{q}_{k_{j}} \in \dot{V}_{n_{\ell}}$ (for $\ell<m, j<\omega$ ). Each sequence $\left\langle p_{k_{j}}^{\ell}: j<\omega\right\rangle \tau$-converges to $p_{\omega}^{\ell}$ so we may find an infinite set $A \subseteq \omega$ and conditions $p_{\ell}^{*} \in U_{\ell}($ for $\ell<m)$ such that

$$
p_{\ell}^{*} \geq p_{\omega}^{\ell} \geq p_{\omega} \quad \text { and } \quad(\forall j \in A)(\forall \ell<m)\left(p_{\ell}^{*} \geq p_{k_{j}}^{\ell}\right) .
$$

Let $X=\left\{k_{j}: j \in A\right\}$.

Claim 4.1.9.3. Suppose $\left\langle\left(p_{n}, \dot{q}_{n}\right): n<\omega\right\rangle \subseteq \mathbb{R}$ is $\tau^{*}$-converging to $\left(p_{\omega}, \dot{q}_{\omega}\right)$. Then there is $X \in[\omega]^{\omega}$ such that:

$(\otimes)$ if $\left(p_{\omega}, \dot{q}_{\omega}\right) \in U^{*}\left(U,\left\langle U_{0}, \ldots, U_{m-1}\right\rangle,\left\langle n_{0}, \ldots, n_{m-1}\right\rangle, \bar{k}\right) \in \mathcal{C}$, then for some $N \in \omega$ and $p_{\ell}^{*} \in U_{\ell}($ for $\ell<m)$ we have:

(i) $p_{\ell}^{*} \geq p_{\omega},(\forall n \in X \backslash N)\left(p_{\ell}^{*} \geq p_{n}\right)$,

(ii) $(\forall n \in X \backslash N)(\forall \ell<m)\left(p_{\ell}^{*} \Vdash \dot{q}_{n} \in \dot{V}_{n_{\ell}}\right)$.

Proof of the claim. Let $\left\langle U_{i}^{*}: i<\omega\right\rangle$ enumerate all sets $U^{*} \in \mathcal{C}$ to which $\left(p_{\omega}, \dot{q}_{\omega}\right)$ belongs. Apply 4.1.9.2 to choose inductively a decreasing sequence $\left\langle X_{i}: i<\omega\right\rangle \subseteq$ $[\omega]^{\omega}$ such that for each $i<\omega$ :

if $U_{i}^{*}=U^{*}\left(U,\left\langle U_{0}, \ldots, U_{m-1}\right\rangle,\left\langle n_{0}, \ldots, n_{m-1}\right\rangle, \bar{k}\right)$, then there are conditions $p_{\ell}^{i} \geq p_{\omega}$ (for $\ell<m$ ) satisfying

$$
(\forall \ell<m)\left(\forall n \in X_{i}\right)\left(p_{\ell}^{i} \in U_{\ell} \& p_{\ell}^{i} \geq p_{n} \& p_{\ell}^{i} \Vdash \dot{q}_{n} \in \dot{V}_{n_{\ell}}\right) .
$$


Next pick an infinite set $X \subseteq \omega$ almost included in all $X_{n}$ 's.

Claim 4.1.9.4. Suppose that $\left\langle\left(p_{n}, \dot{q}_{n}\right): n<\omega\right\rangle \subseteq \mathbb{R} \tau^{*}$-converges to $\left(p_{\omega}, \dot{q}_{\omega}\right) \in$ $U^{*} \in \mathcal{C}$. Then there is a condition $\left(p^{*}, \dot{q}^{*}\right) \in U^{*}$ stronger than $\left(p_{\omega}, \dot{q}_{\omega}\right)$ and such that

$$
\left(\exists^{\infty} n \in \omega\right)\left(\left(p_{n}, \dot{q}_{n}\right) \leq\left(p^{*}, \dot{q}^{*}\right)\right) .
$$

Proof of the claim. Let $U^{*}=U^{*}\left(U,\left\langle U_{0}, \ldots, U_{m-1}\right\rangle,\left\langle n_{0}, \ldots, n_{m-1}\right\rangle,\left\langle k_{0}, \ldots, k_{M}\right\rangle\right)$, and let $p_{\omega}^{\ell} \in U_{\ell}($ for $\ell<m)$ be such that $p_{\omega}^{\ell} \geq p_{\omega}$ and $p_{\omega}^{\ell} \Vdash \dot{q}_{\omega} \in \dot{V}_{n_{\ell}}$. Pick $U^{\prime}, U_{0}^{\prime}, \ldots, U_{m-1}^{\prime} \in \mathcal{U}$ such that

- $p_{\omega} \in U^{\prime} \subseteq U, p_{\omega}^{\ell} \in U_{\ell}^{\prime} \subseteq U_{\ell}$,

- any 3 elements of $U_{\ell}^{\prime}$ have a common upper bound in $U_{\ell}$,

- every element of $U^{\prime}$ has an upper bound in each of $U_{0}^{\prime}, \ldots, U_{m-1}^{\prime}$.

Apply 4.1.9.3 to choose $X \in[\omega]^{\omega}$ such that the condition $(\otimes)$ of 4.1.9.3 holds. Note that then

$$
p_{\omega} \Vdash \text { " the sequence }\left\langle\dot{q}_{n}: n \in X\right\rangle \text { is } \dot{\mathcal{B}} \text {-convergent to } \dot{q}_{\omega} \text { ". }
$$

[Why? If not, then we may pick a condition $r \geq p_{\omega}$ and an integer $N$ such that

$$
r \Vdash \text { " } \dot{q}_{\omega} \in \dot{V}_{N} \&\left(\exists^{\infty} n \in X\right)\left(\dot{q}_{n} \notin \dot{V}_{N}\right) \text { ". }
$$

Pick $W \in \mathcal{U}$ such that $r \in W$ and any 2 members of $W$ are compatible, and apply $(\otimes)$ of 4.1 .9 .3 to $U^{*}\left(U,\langle W\rangle,\langle N\rangle,\left\langle k_{0}, \ldots, k_{M}\right\rangle\right)$. We get a condition $r^{*} \in W$ such that

$$
\left(\forall^{\infty} n \in X\right)\left(r^{*} \geq p_{n} \& r^{*} \Vdash \dot{q}_{n} \in \dot{V}_{N}\right) .
$$

Since $r^{*}, r$ are compatible, we get a contradiction.]

Since $\left\langle p_{n}: n \in X\right\rangle \tau$-converges to $p_{\omega}$, we may find an infinite $X^{\prime} \subseteq X$ and a condition $p^{*} \in U^{\prime}$ such that

$$
p^{*} \geq p_{\omega} \&\left(\forall n \in X^{\prime}\right)\left(p^{*} \geq p_{n}\right) .
$$

Next use $(\nabla)$ of 4.1 .9 .3 to pick conditions $p_{\ell}^{\prime} \in U_{\ell}^{\prime}$ and $N \in \omega$ such that

$$
\left(\forall n \in X^{\prime} \backslash N\right)\left(p_{\ell}^{\prime} \geq p_{n} \text { and } p_{\ell}^{\prime} \Vdash \dot{q}_{n} \in \dot{V}_{n_{\ell}} \quad \text { and } \quad(\forall i \leq M)\left(p_{n} \Vdash \dot{q}_{n} \in \dot{V}_{k_{i}}\right)\right) \text {. }
$$

By the choice of $U^{\prime}, U_{0}^{\prime}, \ldots, U_{m-1}^{\prime}$ we get conditions $p_{\ell}^{*} \in U_{\ell}$ such that $p_{\ell}^{*} \geq p^{*}$, $p_{\ell}^{*} \geq p_{\ell}^{\prime}, p_{\ell}^{*} \geq p_{\omega}^{\ell}($ for $\ell<m)$.

Now we are going to define a $\mathbb{P}$-name $\dot{q}^{*}$ for a condition in $\dot{\mathbb{Q}}$. Let $\mathcal{A}$ be a maximal antichain of $\mathbb{P}$ such that for each $\ell<m$ and $r \in \mathcal{A}$ :

- either $r \geq p_{\ell}^{*}$ or $r, p_{\ell}^{*}$ are incompatible, and

- either $r \geq p^{*}$ or $r, p^{*}$ are incompatible.

Fix $r \in \mathcal{A}$. If $r, p^{*}$ are incompatible, then let $\dot{q}_{r}$ be $\dot{\emptyset}_{\dot{\mathbb{Q}}}$. Assume $r \geq p^{*}$ and let

$$
I=\left\{n_{\ell}: \ell<m \& r \geq p_{\ell}^{*}\right\} \cup\left\{k_{i}: i \leq M\right\} \quad(\neq \emptyset) .
$$

The condition $r$ forces that the sequence $\left\langle\dot{q}_{n}: n \in X^{\prime} \backslash N\right\rangle$ converges to $\dot{q}_{\omega}$, and $\dot{q}_{\omega} \in \bigcap_{j \in I} \dot{V}_{j}$, and $\dot{q}_{n} \in \bigcap_{j \in I} \dot{V}_{j}$ (for all $\left.n \in X^{\prime} \backslash N\right)$. Applying 4.1.6)(i+iii) we find a $\mathbb{P}$-name $\dot{q}_{r}$ for an element of $\dot{\mathbb{Q}}$ such that

$$
r \Vdash \text { " }\left(\forall n \in X^{\prime} \backslash N\right)\left(\dot{q}_{n} \leq \dot{q}_{r} \& \dot{q}_{\omega} \leq \dot{q}_{r} \& \dot{q}_{r} \in \bigcap_{j \in I} \dot{V}_{j}\right) " .
$$

Now, let $\dot{q}^{*}$ be a $\mathbb{P}$-name such that $r \Vdash \dot{q}^{*}=\dot{q}_{r}($ for $r \in \mathcal{A})$. 
Look at the condition $\left(p^{*}, q^{*}\right) \in \mathbb{R}$. Clearly $p^{*} \Vdash \dot{q}^{*} \in \dot{V}_{k_{i}}$ (for all $i \leq M$ ) and $p_{\ell}^{*} \geq p^{*}, p_{\ell}^{*} \Vdash \dot{q}^{*} \in \dot{V}_{n_{\ell}}($ for $\ell<m)$, so $\left(p^{*}, \dot{q}^{*}\right) \in U^{*}$. Moreover, if $n \in\left(X^{\prime} \backslash N\right) \cup\{\omega\}$, then $p^{*} \geq p_{n}$ and $p^{*} \Vdash \dot{q}_{n} \leq \dot{q}$, so $\left(p^{*}, \dot{q}^{*}\right) \geq\left(p_{n}, \dot{q}_{n}\right)$ and we are done.

Claim 4.1.9.5. If $(p, \dot{q}) \in U^{*} \in \mathcal{C}$, then there is $V^{*} \in \mathcal{C}$ such that $(p, \dot{q}) \in V^{*}$ and any two conditions $\left(p_{0}, \dot{q}_{0}\right),\left(p_{1}, \dot{q}_{1}\right) \in V^{*}$ have a common upper bound in $U^{*}$.

Proof of the claim. Let $U^{*}=U^{*}\left(U,\left\langle U_{0}, \ldots, U_{m-1}\right\rangle,\left\langle n_{0}, \ldots, n_{m-1}\right\rangle,\left\langle k_{0}, \ldots, k_{M}\right\rangle\right)$ and let $p_{\ell} \in U_{\ell}$ (for $\ell<m$ ) be such that $p \leq p_{\ell}$ and $p_{\ell} \Vdash \dot{q} \in \dot{V}_{n_{\ell}}$. Pick $U_{\ell}^{\prime} \in \mathcal{U}$ such that $p_{\ell} \in U_{\ell}^{\prime} \subseteq U_{\ell}$ and every three members of $U_{\ell}^{\prime}$ have a common upper bound in $U_{\ell}$. Also choose $U^{\prime}, U^{\prime \prime} \in \mathcal{U}$ such that $p \in U^{\prime \prime} \subseteq U^{\prime} \subseteq U$, and each member of $U^{\prime}$ has an upper bound in every $U_{\ell}^{\prime}\left(\right.$ for $\ell<m$ ) and every two members of $U^{\prime \prime}$ have a common upper bound in $U^{\prime}$. Put

$$
V^{*}=U^{*}\left(U^{\prime \prime},\left\langle U_{0}^{\prime}, \ldots, U_{m-1}^{\prime}\right\rangle,\left\langle n_{0}, \ldots, n_{m-1}\right\rangle,\left\langle k_{0}, \ldots, k_{M}\right\rangle\right) \in \mathcal{C} .
$$

Clearly $(p, \dot{q}) \in V^{*}$. Suppose now that $\left(p_{0}, \dot{q}_{0}\right),\left(p_{1}, \dot{q}_{1}\right) \in V^{*}$ and let $p_{\ell}^{i} \in U_{\ell}^{\prime}$ be such that $p_{\ell}^{i} \Vdash \dot{q}_{i} \in \dot{V}_{n_{\ell}}$ and $p_{i} \leq p_{\ell}^{i}$ (for $i=0,1$ and $\ell<m$ ). Also let $p^{*} \in U^{\prime}$ be stronger than both $p_{0}$ and $p_{1}$, and for each $\ell<m$ let $p_{\ell}^{*} \in U_{\ell}$ be stronger than both $p^{*}$ and $p_{\ell}^{0}$ and $p_{\ell}^{1}$. Now, like in the proof of 4.1.9.4, choose a $\mathbb{P}$-name $\dot{q}^{*}$ for a condition in $\dot{\mathbb{Q}}$ such that

$$
\begin{aligned}
& p^{*} \Vdash " \dot{q}^{*} \geq \dot{q}_{0} \& \dot{q}^{*} \geq \dot{q}_{1} ", \text { and } \\
& p_{\ell}^{*} \Vdash " \dot{q}^{*} \in \dot{V}_{n_{\ell}} " \text { for } \ell<m, \text { and } \\
& p^{*} \Vdash " \dot{q}^{*} \in \dot{V}_{k_{\ell}} " \text { for } \ell \leq M .
\end{aligned}
$$

(Remember that the sets $\bigcap_{j \in I} \dot{V}_{j}$ are forced to be directed by 4.1.6(i+ii).) Then $\left(p^{*}, \dot{q}^{*}\right) \in U^{*}$ is a condition stronger than both $\left(p_{0}, \dot{q}_{0}\right)$ and $\left(p_{1}, \dot{q}_{1}\right)$.

Now we may put together 4.1.9.4, 4.1.9.1(2) and 4.1.9.5 to conclude that the topology $\tau^{*}$ satisfies the demand 4.1.3(iii), finishing the proof of the theorem.

Note that $\left(p, \dot{\emptyset}_{\dot{\mathbb{Q}}}\right) \in \mathbb{R}$ for each $p \in \mathbb{P}$ and the mapping $p \mapsto\left(p, \dot{\emptyset}_{\dot{\mathbb{Q}}}\right)$ is a homeomorphic embedding of $(\mathbb{P}, \tau)$ into $\left(\mathbb{R}, \tau^{*}\right)$, so we may think that $\tau$ is the restriction of $\tau^{*}$ to $\mathbb{P} \subseteq \mathbb{R}$. Moreover, under this interpretation, $\mathbb{P}$ is an open subset of $\mathbb{R}$.

Let us state (without a proof) a result that shows how 4.1.9 (together with 4.1.5) can be applied.

Context 4.1.10. Let $\mathcal{K}$ be a collection of tuples $\bar{a}=\left\langle\varphi_{0}, \varphi_{1}, \varphi_{2}, h, W\right\rangle$, where $\varphi_{i}$ are $\Sigma_{1}^{1}$ formulas and $h, W$ are Borel functions such that for each real $r$ :

- $\varphi_{0}(\cdot, r), \varphi_{1}(\cdot, r), \varphi_{2}(\cdot, r)$ define a Souslin ccc forcing notion $\mathbb{P}^{\bar{a}, r}$ (and $\leq_{\mathbb{P} a \bar{a}, r}$, $\perp_{\left.\mathbb{P}^{\bar{a}, r}\right)}$,

- $h(r)$ is a function $\mathbf{H}^{\bar{a}, r}: \omega \longrightarrow \mathcal{H}\left(\omega_{1}\right)$ (and we have $\mathcal{T}_{n}^{\bar{a}, r}=\prod_{i<n} \mathbf{H}^{\bar{a}, r}(i)$ and $\left.\mathcal{T}^{\bar{a}, r}=\bigcup_{n<\omega} \mathcal{T}_{n}\right)$,

- $W(r)$ is a sequence $\dot{W}^{\bar{a}, r}=\left\langle p_{\eta}: \eta \in \mathcal{T}^{\bar{a}, r}\right\rangle \subseteq \mathbb{P}^{\bar{a}, r}$,

- $\mathbb{P}^{\bar{a}, r}, \mathbf{H}^{\bar{a}, r}, \dot{W}^{\bar{a}, r}$ are as in 3.1.1.

$\mathcal{K}_{0}, \mathcal{K}_{1}$ are subsets of $\mathcal{K}$ such that

- for each $\bar{a} \in \mathcal{K}_{0}$ and every real $r$, the forcing notion $\mathbb{P}^{\bar{a}, r}$ is iterably sweet, 
- for each $\bar{a} \in \mathcal{K}_{1}$ and a real $r$, there is an iterably sweet Souslin ccc forcing notion $\mathbb{Q}_{\bar{a}, r}$ such that

$$
\begin{aligned}
& \Vdash_{\mathbb{Q}_{\bar{a}, r}} \text { " the union of all Borel sets from the ideal } \mathcal{I}_{\mathbb{P} \bar{a}, r, \dot{W}^{\bar{a}, r}} \\
& \text { coded in } \mathbf{V} \text { belongs to the ideal } \mathcal{I}_{\mathbb{P}^{\bar{a}, r}, \dot{W}^{\bar{a}, r}} " .
\end{aligned}
$$

Theorem 4.1.11. Assume GCH. Let $\mathcal{K}, \mathcal{K}_{0}, \mathcal{K}_{1}$ be as in 4.1 .10 and $\kappa=\kappa^{<\kappa}$. Then there is a forcing notion $\mathbb{Q}$ preserving cofinalities and cardinalities and such that for every generic $G \subseteq \mathbb{Q}$ over $\mathbf{V}$ we have (in $\mathbf{V}[G]$ ):

1. $\mathfrak{c}=\kappa$,

2. for every real $r$ and $\bar{a} \in \mathcal{K}_{1}$, every projective subset of $\prod_{n<\omega} \mathbf{H}^{\bar{a}, r}(n)$ has $\mathcal{I}_{\mathbb{P} \bar{a}, r, \dot{W} \bar{a}, r}$-Baire property,

3. for each real $r$, a sequence $\left\langle r_{\alpha}: \alpha<\mu\right\rangle$ of reals, $\mu<\kappa$, and $\bar{a} \in \mathcal{K}_{0}$ there is

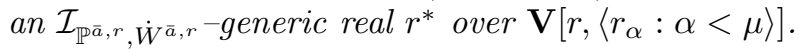

Proof. See 19.

Proposition 4.1.12. 1. In the cases discussed in 1.1.14 (1,2), the forcing notion under considerations is topologically sweet, provided $K$ is countable.

2. If $(K, \Sigma)$ is a really finitary (see 1.3. 3) linked tree-creating pair, and $f$ is a fast function, then the forcing notion $\mathbb{Q}_{f}^{\text {tree }}(K, \Sigma)$ is topologically sweet.

3. Let $\mathfrak{p}=(K, \Sigma, \mathcal{F}, \mathcal{G})$ be a universality parameter for $\mathbf{H}$. Assume that

(a) $\mathcal{F}$ is either countable or $<\omega_{1}-\leq^{*}$-directed, and

(b) for each $\eta \in \bigcup_{n<\omega} \prod_{i<n} \mathbf{H}(i)$ there is $t_{\eta}^{\max } \in \operatorname{LTCR}_{\eta}[\mathbf{H}] \cap K$ such that

$$
\left(\forall t \in \operatorname{LTCR}_{\eta}[\mathbf{H}] \cap K\right)\left(t \in \Sigma\left(t_{\eta}^{\max }\right)\right) .
$$

Then the forcing notion $\mathbb{Q}^{\text {tree }}(\mathfrak{p})$ is sweet (and thus iterably sweet, provided elements compatible in $\mathbb{Q}^{\text {tree }}(\mathfrak{p})$ have the least upper bound).

Proof. 1) Let $\left(K, \Sigma, \Sigma^{\perp}\right)$ be a $\otimes$-creating triple for $\mathbf{H}: \omega \longrightarrow \mathcal{H}\left(\omega_{1}\right)$. Suppose that $\left(K, \Sigma, \Sigma^{\perp}\right)$ is linked, gluing and has the cutting property.

For $\mathbf{c}=\left(w, t_{0}, \ldots, t_{n}\right) \in \mathrm{FC}\left(K, \Sigma, \Sigma^{\perp}\right)$ and $N<\omega$ let

$$
\begin{aligned}
& U(\mathbf{c}, N)=\left\{p \in \mathbb{Q}_{\infty}^{*}\left(K, \Sigma, \Sigma^{\perp}\right): \quad w^{p}=w \quad \& \quad(\forall k \leq n)\left(t_{k}^{p}=t_{k}\right) \quad \&\right. \\
& \left.(\forall k>n)\left(\operatorname{nor}\left[t_{k}^{p}\right] \geq N\right)\right\} \text {. }
\end{aligned}
$$

Let $\tau$ be the topology generated by the sets $U(\mathbf{c}, N)$ (for $\mathbf{c} \in \mathrm{FC}\left(K, \Sigma, \Sigma^{\perp}\right.$ ) and $N<\omega)$ and $\left\{\emptyset_{\mathbb{Q}_{\infty}^{*}\left(K, \Sigma, \Sigma^{\perp}\right)}\right\}$. It is straightforward to check that $\left(\mathbb{Q}_{\infty}^{*}\left(K, \Sigma, \Sigma^{\perp}\right), \tau\right)$ is a model of topological sweetness.

Other instances of 1) and 2) can be handled similarly.

3) We consider the case when $\mathcal{F}$ is countable only (if $\mathcal{F}$ is $<\omega_{1}-\leq^{*}$-directed the proof is similar). We put $\mathcal{D}=\mathbb{Q}^{\text {tree }}(\mathfrak{p})$ and we define relations $E_{n}$ (for $\left.n<\omega\right)$ on $\mathcal{D}$ as follows:

$$
\begin{aligned}
& p_{0} E_{n} p_{1} \quad \text { if and only if } \\
& N^{p_{0}}=N^{p_{1}}, f^{p_{0}}=f^{p_{1}} \text { and } \\
& \qquad\left(\forall \eta \in T^{p_{0}}\right)\left(\ln (\eta)<N^{p_{0}}+n \Rightarrow \eta \in T^{p_{1}} \& t^{p_{0}}=t^{p_{1}}\right) .
\end{aligned}
$$

We claim that $\left(\mathbb{Q}^{\text {tree }}(\mathfrak{p}), \mathcal{D},\left\langle E_{n}: n<\omega\right\rangle\right)$ is a model of sweetness. Plainly, each $E_{n}$ is an equivalence relation with countably many equivalence classes, $E_{n+1} \subseteq E_{n}$. Similarly as in 2.3.6.1 one can show that the equivalence classes of each $E_{n}$ are 
directed and that the clause 1.1.2(v) is satisfied. Let us show that the demand 4.1.2(iv) holds.

So suppose $p_{i} E_{i} p_{\omega}$ (for $i \geq n, n<\omega$ ). Thus $f^{p_{i}}=f^{p_{\omega}}=f, N^{p_{i}}=N^{p_{\omega}}=N$ (for $i \geq n$ ). We build inductively a system $\left\langle t_{\eta}: \eta \in T\right\rangle \in \mathbb{Q}_{\emptyset}^{\text {tree }}(K, \Sigma)$ as follows.

First we let $M_{0}=N+n$ and we declare

$$
\eta \in T^{p_{\omega}} \& \operatorname{lh}(\eta)<M_{0} \Rightarrow \eta \in T \& t_{\eta}=t_{\eta}^{p_{\omega}} .
$$

Suppose that we have defined $\left\langle t_{\eta}: \eta \in T \& \operatorname{lh}(\eta)<M_{k}\right\rangle \in \operatorname{FC}(K, \Sigma)$ already. Pick $M_{k}^{\prime}>M_{k}$ such that for some $n_{\mathrm{dn}}^{k}, n_{\mathrm{up}}^{k}$ we have $M_{k}<n_{\mathrm{dn}}^{k} \leq n_{\mathrm{up}}^{k} \leq F^{\mathcal{G}}\left(n_{\mathrm{up}}^{k}\right)<M_{k}^{\prime}$ and

$$
\left(\left\langle t_{\eta}^{p_{\omega}}: \eta \in T^{p_{\omega}} \& \operatorname{lh}(\eta)<M_{k}^{\prime}\right\rangle, n_{\mathrm{dn}}^{k}, n_{\mathrm{up}}^{k}, f\left\lceil\left[n_{\mathrm{dn}}^{k}, n_{\mathrm{up}}^{k}\right]\right) \in \mathcal{G} .\right.
$$

Next, choose $M_{k+1}$ and $n_{\mathrm{dn}}^{k, i}, n_{\mathrm{up}}^{k, i}$ (for $i \in\left[n, M_{k}^{\prime}\right]$ ) such that $M_{k}^{\prime}<n_{\mathrm{dn}}^{k, n}, n_{\mathrm{dn}}^{k, i} \leq$ $n_{\mathrm{up}}^{k, i} \leq F^{\mathcal{G}}\left(n_{\mathrm{up}}^{k, i}\right)<n_{\mathrm{dn}}^{k, i+1}-2, F^{\mathcal{G}}\left(n_{\mathrm{up}}^{k, M_{k}^{\prime}}\right)<M_{k+1}$ and

$$
\left(\left\langle t_{\eta}^{p_{i}}: \eta \in T^{p_{i}} \& \operatorname{lh}(\eta)<M_{k+1}\right\rangle, n_{\mathrm{dn}}^{k, i}, n_{\mathrm{up}}^{k, i}, f \uparrow\left[n_{\mathrm{dn}}^{k, i}, n_{\mathrm{up}}^{k, i}\right]\right) \in \mathcal{G} .
$$

Let $\left\langle t_{\eta}^{k}: \eta \in \hat{S}_{k}\right\rangle \in \mathrm{FC}(K, \Sigma)$ be such that $\operatorname{root}\left(S_{k}\right)=\langle\rangle, \operatorname{lev}\left(S_{k}\right)=M_{k+1}$, and $t_{\eta}^{k}=t_{\eta}^{p_{\omega}}$ when $\operatorname{lh}(\eta)<M_{k}^{\prime}$ and $t_{\eta}^{k}=t_{\eta}^{\max }$ when $M_{k}^{\prime} \leq \operatorname{lh}(\eta)<\operatorname{lev}\left(S_{k}\right)$. Apply repeatedly 2.3.3 $(\varepsilon)$ to get $\left\langle t_{\eta}: \eta \in T \& \operatorname{lh}(\eta)<M_{k+1}\right\rangle \in \mathrm{FC}(K, \Sigma)$ such that

$$
\left(\left\langle t_{\eta}: \eta \in T \& \operatorname{lh}(\eta)<M_{k+1}\right\rangle, n_{\mathrm{dn}}^{k}, n_{\mathrm{up}}^{k, M_{k}^{\prime}}, f \uparrow\left[n_{\mathrm{dn}}^{k}, n_{\mathrm{up}}^{k, M_{k}^{\prime}}\right]\right) \in \mathcal{G},
$$

and $\left\langle t_{\eta}: \eta \in T \& \operatorname{lh}(\eta)<M_{k+1}\right\rangle \leq\left\langle t_{\eta}^{k}: \eta \in \hat{S}_{k}\right\rangle$, and

$$
\left\langle t_{\eta}: \eta \in T \& \operatorname{lh}(\eta)<M_{k+1}\right\rangle \leq\left\langle t_{\eta}^{p_{i}}: \eta \in T^{p_{i}}\right\rangle \quad \text { for all } i \in\left[n, M_{k}^{\prime}\right] .
$$

Note that then $\left\langle t_{\eta}: \eta \in T \& \operatorname{lh}(\eta)<M_{k+1}\right\rangle \leq\left\langle t_{\eta}^{p_{i}}: \eta \in T^{p_{i}}\right\rangle$ for all $i \in[n, \omega]$.

After the construction is carried out one easily checks that $q=\left(N,\left\langle t_{\eta}: \eta \in\right.\right.$ $T\rangle, f) \in \mathbb{Q}^{\text {tree }}(\mathfrak{p})$ is a condition stronger than all $p_{i}$ 's (for $\left.i \in[n, \omega]\right)$ and $q E_{n} p_{\omega}$.

Definition 4.1.13. Suppose that a function $h: \omega \times \omega \longrightarrow \omega$ is regressive, $\mathcal{F} \subseteq$ $(\omega \backslash 2)^{\omega}$, and $(K, \Sigma)$ is a local creating pair for $\mathbf{H}$. We define $\mathbb{Q}_{\mathcal{F}}^{h}(K, \Sigma)$ as the suborder of $\mathbb{Q}_{\mathcal{F}}^{*}(K, \Sigma)$ consisting of conditions $p \in \mathbb{Q}_{\mathcal{F}}^{*}(K, \Sigma)$ such that

$$
(\exists f \in \mathcal{F})(\forall k<\omega)\left(\forall^{\infty} n<\omega\right)\left(h^{(k)}\left(m^{t_{n}^{p}}, \operatorname{nor}\left[t_{n}^{p}\right]\right) \geq f\left(m^{t_{n}^{p}}\right)\right),
$$

where $h^{(k+1)}(i, j)=h^{(k)}(i, h(i, j)), h^{(0)}(i, j)=j$.

Remark 4.1.14. Note that the norm condition introduced in 4.1.13 is in many cases nothing new. If for each $f \in \mathcal{F}$ there is $f^{+} \in \mathcal{F}$ such that

$$
(\forall k<\omega)\left(\forall^{\infty} n<\omega\right)\left(h^{(k)}(n, f(n)) \geq f^{+}(n)\right),
$$

then clearly $\mathbb{Q}_{\mathcal{F}}^{*}(K, \Sigma)=\mathbb{Q}_{\mathcal{F}}^{h}(K, \Sigma)$.

Proposition 4.1.15. Let $h: \omega \times \omega \longrightarrow \omega$ be regressive and $\mathcal{F} \subseteq(\omega \backslash 2)^{\omega}$ be a countable family. Assume that $(K, \Sigma)$ is a local h-linked creating pair for $\mathbf{H}$ such that $K$ is countable and

$(*)$ if $s, t \in K$ and $s \in \Sigma(t)$, then $\operatorname{nor}[s] \leq \operatorname{nor}[t]$.

Then the forcing notion $\mathbb{Q}_{\mathcal{F}}^{h}(K, \Sigma)$ is topologically sweet. 
Proof. For a finite candidate $\mathbf{c}=\left(w, t_{0}, \ldots, t_{m}\right) \in \mathrm{FC}(K, \Sigma)$ and sequences $\bar{f}=$ $\left\langle f_{\ell}: \ell \leq \ell^{*} \subseteq \mathcal{F}\right.$ and $\bar{k}=\left\langle k_{\ell}: \ell \leq \ell^{*}\right\rangle \subseteq \omega$ we let

$$
\begin{aligned}
& U(\mathbf{c}, \bar{f}, \bar{k})=\left\{p \in \mathbb{Q}_{\mathcal{F}}^{h}(K, \Sigma): w^{p}=w \quad \& \quad(\forall n \leq m)\left(t_{n}^{p}=t_{n}\right) \quad \&\right. \\
&\left(\forall \ell \leq \ell^{*}\right)(\forall n>m)\left(h^{\left(k_{\ell}\right)}\left(m_{\mathrm{dn}}^{t_{n}^{p}}, \operatorname{nor}\left[t_{n}^{p}\right]\right) \geq f_{\ell}\left(m^{t_{n}^{p}}\right)\right) \quad \& \\
&\left.\left(\forall \ell \leq \ell^{*}\right)(\forall k<\omega)\left(\forall^{\infty} n<\omega\right)\left(h^{(k)}\left(m_{\mathrm{dn}}^{t_{n}^{p}}, \operatorname{nor}\left[t_{n}^{p}\right]\right) \geq f_{\ell}\left(m^{t_{n}^{p}}\right)\right)\right\} .
\end{aligned}
$$

Plainly the sets $U(\mathbf{c}, \bar{f}, \bar{k})$ (for suitable $\mathbf{c}, \bar{f}, \bar{k}$ ) and $\left\{\emptyset_{\mathbb{Q}_{\mathcal{F}}^{h}(K, \Sigma)}\right\}$ constitute a basis of a topology $\tau$ on $\mathbb{Q}_{\mathcal{F}}^{h}(K, \Sigma)$. it is not difficult to check that $\left(\mathbb{Q}_{\mathcal{F}}^{h}(K, \Sigma), \tau\right)$ is a model of topological sweetness.

4.2. The sour part of the spectrum. The main point of the sweet properties is that amalgamations of sweet forcing notions are ccc, see 4.1.5. A kind of opposite behaviour is when amalgamating results in a forcing notion that collapses $\omega_{1}$. This effect will be called sourness, and we have a number of variants of it (see 4.2.2 below).

In this part, whenever we use $\mathbb{Q}$-names for elements of a space $\mathcal{X}=\prod_{i<\omega} \mathbf{H}(i)$, we assume that they are in a standard form. Thus, a $\mathbb{Q}$-name $\dot{\tau}$ for a real in $\mathcal{X}$ is a system

$$
\left\langle q_{\eta, k}^{n}: n<\omega \& \eta \in \prod_{i<n} \mathbf{H}(i) \& k<N_{\eta}\right\rangle,
$$

where $q_{\eta, k}^{n} \in \mathbb{Q}, N_{\eta} \leq \omega$ and for each $n<\omega$

$$
\left\langle q_{\eta, k}^{n}: \eta \in \prod_{i<n} \mathbf{H}(i) \& k<N_{\eta}\right\rangle
$$

is a maximal antichain of $\mathbb{Q}$, and

$$
\nu \triangleleft \eta \quad \Rightarrow \quad\left(\forall k<N_{\eta}\right)\left(\exists m<N_{\nu}\right)\left(q_{\nu, m}^{\operatorname{lh}(\nu)} \leq q_{\eta, k}^{\mathrm{lh}(\eta)}\right) .
$$

(The intension is that $q_{\eta, k}^{n} \Vdash \eta \triangleleft \dot{\tau}$.) If ( $\mathbb{Q}$ is ccc and) $\dot{\tau}_{0}$ is a $\mathbb{Q}$-name for an element of $\mathcal{X}$ then there is a standard name $\dot{\tau}$ such that $\Vdash \dot{\tau}_{0}=\dot{\tau}$. The point of using standard names is that their specific forms allows us to bound the complexity of some formulas; see, e.g., [1, 3.6.12].

Context 4.2.1. Let $\mathbb{P}, \dot{W}, \mathcal{X}$ be as in 3.1.1, and let $\mathbb{Q}_{0}, \mathbb{Q}_{1}$ be Souslin ccc forcing notions. Suppose that, for $\ell<2, \dot{\tau}_{\ell}$ is a standard $\mathbb{Q}_{\ell}$-name for an element of $\mathcal{X}$. Furthermore, suppose that there are isomorphisms $f_{\ell}: \operatorname{RO}(\mathbb{P}) \stackrel{\text { onto }}{\longrightarrow}\left(\operatorname{RO}\left(\mathbb{Q}_{\ell}\right)\right)_{\dot{\tau}_{\ell}}$ mapping $\dot{W}$ onto $\dot{\tau}_{\ell}$ (i.e., $f_{\ell}\left(\llbracket \eta \triangleleft \dot{W} \rrbracket_{\mathrm{RO}(\mathbb{P})}\right)=\llbracket \eta \triangleleft \dot{\tau}_{\ell} \rrbracket_{\mathrm{RO}\left(\mathbb{Q}_{\ell}\right)}$ ).

Let $r$ be a real encoding all parameters required for the definitions of the above objects (including partial orders and the respective incompatibility relations).

Definition 4.2.2. Let $\mathbb{P}, \dot{W}, \mathcal{X}, \mathbb{Q}_{i}, \dot{\tau}_{\ell}, f_{\ell}, r$ be as in 4.2.1.

(a) The amalgamation $\mathbb{Q}_{0} \times_{f_{0}, f_{1}} \mathbb{Q}_{1}$ will be also denoted $\mathbb{Q}_{0} \times_{\dot{\tau}_{0}=\dot{\tau}_{1}} \mathbb{Q}_{1}$.

We say that

(b) $\left(\mathbb{Q}_{0}, \dot{\tau}_{0}\right)$ is weakly sour to $\left(\mathbb{Q}_{1}, \dot{\tau}_{1}\right)$ if the amalgamation $\mathbb{Q}_{0} \times_{\dot{\tau}_{0}=\dot{\tau}_{1}} \mathbb{Q}_{1}$ fails the ccc;

(c) $\left(\mathbb{Q}_{0}, \dot{\tau}_{0}\right)$ is sour to $\left(\mathbb{Q}_{1}, \dot{\tau}_{1}\right)$ whenever the following condition holds:

$(\boxplus)$ if $\mathbf{V} \subseteq \mathbf{V}^{\prime}$ are universes of ZFC, $r \in \mathbf{V}, G_{0}, G_{1} \in \mathbf{V}^{\prime}, G_{\ell} \subseteq \mathbb{Q}_{\ell} \mathbf{V}$ is generic over $\mathbf{V}$ and $\dot{\tau}_{0}^{G_{0}}=\dot{\tau}_{1}^{G_{1}}, \quad$ then $\mathbf{V}^{\prime} \models " \omega_{1}^{\mathbf{V}}$ is countable "; 
(d) $\left(\mathbb{Q}_{0}, \dot{\tau}_{0}\right)$ is explicitly sour to $\left(\mathbb{Q}_{1}, \dot{\tau}_{1}\right)$ if there are sequences $\left\langle E_{m}: m<\omega\right\rangle$ and $\left\langle q_{\alpha, n}^{\ell}: \alpha<\omega_{1} \& n<\omega\right\rangle$ (for $\ell<2$ ) such that

(i) each $E_{m}$ is an equivalence relation on $\omega_{1}$ with at most countably many equivalence classes,

(ii) $\left\{q_{\alpha, n}^{\ell}: n<\omega\right\} \subseteq \mathbb{Q}_{\ell}$ is predense in $\mathbb{Q}_{\ell}$ (for each $\alpha<\omega_{1}, \ell<2$ ),

(iii) if $\alpha<\beta<\omega_{1}, m<\omega, \alpha E_{m} \beta$ and $n_{0}, n_{1}<m$ and both $\left(q_{\alpha, n_{0}}^{0}, q_{\alpha, n_{1}}^{1}\right)$ and $\left(q_{\beta, n_{0}}^{0}, q_{\beta, n_{1}}^{1}\right)$ are in the amalgamation $\mathbb{Q}_{0} \times_{\dot{\tau}_{0}=\dot{\tau}_{1}} \mathbb{Q}_{1}$, then the conditions $\left(q_{\alpha, n_{0}}^{0}, q_{\alpha, n_{1}}^{1}\right),\left(q_{\beta, n_{0}}^{0}, q_{\beta, n_{1}}^{1}\right)$ are incompatible (in $\left.\mathbb{Q}_{0} \times_{\dot{\tau}_{0}=\dot{\tau}_{1}} \mathbb{Q}_{1}\right)$;

(e) $\left(\mathbb{Q}_{0}, \dot{\tau}_{0}\right)$ is very explicitly sour to $\left(\mathbb{Q}_{1}, \dot{\tau}_{1}\right)$ if there are Borel functions $g_{\ell}$ : $\mathcal{X} \times \omega \longrightarrow \mathbb{Q}_{\ell}$ such that

(i) $\left\{g_{\ell}(x, n): n<\omega\right\}$ is predense in $\mathbb{Q}_{\ell}$ (for each $x \in \mathcal{X}, \ell<2$ ),

(ii) if $x_{0}, x_{1} \in \mathcal{X}$ are distinct, $x_{0}\left\lceil m=x_{1}\left\lceil m, k_{0}, k_{1}<m\right.\right.$, then there are $n<\omega$ and disjoint sets $A_{0}, A_{1} \subseteq \prod_{i<n} \mathbf{H}(i)$ such that for $\ell<2$ :

$\left(\forall q \in \mathbb{Q}_{\ell}\right)\left(\left[q \geq g_{\ell}\left(x_{0}, k_{\ell}\right) \& q \geq g_{\ell}\left(x_{1}, k_{\ell}\right)\right] \quad \Rightarrow \quad q \Vdash \dot{\tau}_{\ell}\left\lceil n \in A_{\ell}\right)\right.$.

We say that $\mathbb{Q}_{0}$ is sour to $\mathbb{Q}_{1}$ over $(\mathbb{P}, \dot{W})$ if there are names $\dot{\tau}_{0}, \dot{\tau}_{1}$ (as above) such that $\left(\mathbb{Q}_{0}, \dot{\tau}_{0}\right)$ is sour to $\left(\mathbb{Q}_{1}, \dot{\tau}_{1}\right)$ (and similarly for the variants).

A forcing notion $\mathbb{Q}$ is sour over $(\mathbb{P}, \dot{W})$ if it is sour to itself over $(\mathbb{P}, \dot{W})$ (and similarly for the other notions).

We may skip $\dot{W}$ and say "over $\mathbb{P}$ " if it is clear what $\dot{W}$ we consider.

Remark 4.2.3. Sourness (4.2.2(c)) is a strong way to say that the amalgamation $\mathbb{Q}_{0} \times_{\dot{\tau}_{0}=\dot{\tau}_{1}} \mathbb{Q}_{1}$ collapses $\omega_{1}$. Explicit sourness $(4.2 .2(\mathrm{~d}))$ guarantees that we have a nice witness for the collapse, see 4.2.4 below. What is the point of "very explicitly sour"? On one hand that condition implies that the amalgamation collapses the continuum, and on the other hand the name for the collapsing function is encoded in a nice way by a real. Note that the properties of the functions $g_{0}, g_{1}$ required in $4.2 .2(\mathrm{e})(\mathrm{i}, \mathrm{ii})$ are $\Pi_{1}^{1}$ (remember that $\dot{\tau}_{\ell}$ are standard names), and thus we have suitable absoluteness.

Proposition 4.2.4. Let $\left(\mathbb{Q}_{0}, \dot{\tau}_{0}\right),\left(\mathbb{Q}_{1}, \dot{\tau}_{1}\right),(\mathbb{P}, \dot{W})$ and $r$ be as in 4.2.1.

1. If $\left(\mathbb{Q}_{0}, \dot{\tau}_{0}\right)$ is explicitly sour to $\left(\mathbb{Q}_{1}, \dot{\tau}_{1}\right)$ in every universe $\mathbf{V}$ of $\mathrm{ZFC}$ containing $r$, then $\left(\mathbb{Q}_{0}, \dot{\tau}_{0}\right)$ is sour to $\left(\mathbb{Q}_{1}, \dot{\tau}_{1}\right)$.

2. If $\left(\mathbb{Q}_{0}, \dot{\tau}_{0}\right)$ is very explicitly sour to $\left(\mathbb{Q}_{1}, \dot{\tau}_{1}\right)$ with functions $g_{0}, g_{1}$ witnessing this, then $\left(\mathbb{Q}_{0}, \dot{\tau}_{0}\right)$ is explicitly sour to $\left(\mathbb{Q}_{1}, \dot{\tau}_{1}\right)$ in every universe of ZFC containing $r$ and (the Borel codes for) $g_{0}, g_{1}$.

Proof. 1) Suppose that $\mathbf{V} \subseteq \mathbf{V}^{\prime}$ are universes of ZFC, $r \in \mathbf{V}, G_{0}, G_{1} \in \mathbf{V}^{\prime}$, $G_{\ell} \subseteq \mathbb{Q}_{\ell}^{\mathbf{V}}$ is generic over $\mathbf{V}$ and $\dot{\tau}_{0}^{G_{0}}=\dot{\tau}_{1}^{G_{1}}$.

Assume $\mathbf{V}^{\prime} \models \omega_{1}^{\mathbf{V}}=\omega_{1}$.

Claim 4.2.4.1. 1. If $q_{\ell} \in \mathbb{Q}_{\ell}$ and $\left(q_{0}, q_{1}\right) \notin \mathbb{Q}_{0} \times_{\dot{\tau}_{0}=\dot{\tau}_{1}} \mathbb{Q}_{1}$, then for some disjoint Borel sets $B_{0}, B_{1} \subseteq \mathcal{X}$ we have $q_{0} \Vdash \dot{\tau}_{0} \in B_{0}$ and $q_{1} \Vdash \dot{\tau}_{1} \in B_{1}$. Consequently, if $q_{0} \in G_{0}$ and $q_{1} \in G_{1}$, then $\left(q_{0}, q_{1}\right) \in \mathbb{Q}_{0} \times_{\dot{\tau}_{0}=\dot{\tau}_{1}} \mathbb{Q}_{1}$.

2. If $\left(q_{0}, q_{1}\right),\left(q_{0}^{\prime}, q_{1}^{\prime}\right) \in \mathbb{Q}_{0} \times_{\dot{\tau}_{0}=\dot{\tau}_{1}} \mathbb{Q}_{1}$ are incompatible in $\mathbb{Q}_{0} \times_{\dot{\tau}_{0}=\dot{\tau}_{1}} \mathbb{Q}_{1}$, then for some disjoint Borel sets $B_{0}, B_{1} \subseteq \mathcal{X}$ we have (for $\ell=0,1$ ):

$$
\left(\forall p \in \mathbb{Q}_{\ell}\right)\left(p \geq q_{\ell} \& p \geq q_{\ell}^{\prime} \quad \Rightarrow \quad p \Vdash \dot{\tau}_{\ell} \in B_{\ell}\right) .
$$

Proof of the claim. Straightforward if you remember 3.1.6. 
Now, let $\left\langle E_{m}: m<\omega\right\rangle,\left\langle q_{\alpha, k}^{\ell}: \alpha<\omega_{1}^{\mathbf{V}}, k<\omega\right\rangle \in \mathbf{V}$ witness that $\left(\mathbb{Q}_{0}, \dot{\tau}_{0}\right)$ is explicitly sour to $\left(\mathbb{Q}_{1}, \dot{\tau}_{1}\right)$ (in $\mathbf{V}$ ). By 4.2.2(d)(ii) we know that, in $\mathbf{V}^{\prime}$,

$$
\left(\forall \alpha<\omega_{1}\right)\left(\exists k_{\alpha}^{0}, k_{\alpha}^{1}<\omega\right)\left(q_{\alpha, k_{\alpha}^{0}}^{0} \in G_{0} \& q_{\alpha, k_{\alpha}^{1}}^{1} \in G_{1}\right) .
$$

For some $k^{0}, k^{1}<\omega$ the set

$$
Y=\left\{\alpha<\omega_{1}: k_{\alpha}^{0}=k^{0} \& k_{\alpha}^{1}=k^{1}\right\}
$$

is uncountable. Let $m=k^{0}+k^{1}+1$. It follows from 4.2.2(d)(i) that there are distinct $\alpha, \beta \in Y$ such that $\alpha E_{m} \beta$. By 4.2.4.1 (1) we know $\left(q_{\alpha, k^{0}}^{0}, q_{\alpha, k^{1}}^{1}\right),\left(q_{\beta, k^{0}}^{0}, q_{\beta, k^{1}}^{1}\right) \in$ $\mathbb{Q}_{0} \times_{\dot{\tau}_{0}=\dot{\tau}_{1}} \mathbb{Q}_{1}$, so by 1.2 .2 (d) (iii) these two conditions are incompatible in $\mathbb{Q}_{0} \times_{\dot{\tau}_{0}=\dot{\tau}_{1}}$ $\mathbb{Q}_{1}$. But then, using 4.2.4.1 (2), we find disjoint Borel sets $B_{0}, B_{1} \subseteq \mathcal{X}$ such that $\dot{\tau}_{0}^{G_{0}} \in B_{0}$ and $\dot{\tau}_{1}^{G_{1}} \in B_{1}$, a contradiction to $\dot{\tau}_{0}^{G_{0}}=\dot{\tau}_{1}^{G_{1}}$.

2) Let $\mathbf{V}$ contain $r$ and $g_{0}, g_{1}$. Working in $\mathbf{V}$, pick a sequence $\left\langle x_{\alpha}: \alpha<\omega_{1}\right\rangle$ of pairwise distinct members of $\mathcal{X}$. Put:

- $q_{\alpha, n}^{0}=g_{0}\left(x_{\alpha}, n\right), q_{\alpha, n}^{1}=g_{1}\left(x_{\alpha}, n\right)$,

- $\alpha E_{m} \beta$ if and only if $x_{\alpha}\left\lceil m=x_{\beta}\lceil m\right.$.

Now check.

Proposition 4.2.5. Let $r, \mathbb{P}, \dot{W}, \mathbb{Q}_{\ell}, \dot{\tau}_{\ell}($ for $\ell<2)$ be as in 4.2.1. Moreover, let $\mathbb{Q}_{\ell}, \dot{W}_{\ell}, \mathcal{X}_{\ell}, \mathbf{H}_{\ell}($ for $\ell<2)$ be as in 3.1.1 (with the real $r$ encoding needed parameters). Assume that

(a) $\omega_{1}$ is not an inaccessible cardinal in $\mathbf{L}$,

(b) $\left(\mathbb{Q}_{0}, \dot{\tau}_{0}\right)$ is sour to $\left(\mathbb{Q}_{1}, \dot{\tau}_{1}\right)$,

(c) for every real $s$ and a Borel set $B \subseteq \mathcal{X}_{\ell}$ coded in $\mathbf{L}[r, s]$, if $B \notin \mathcal{I}_{\mathbb{Q}_{\ell}, \dot{W}_{\ell}}$ then there is an $\mathcal{I}_{\mathbb{Q}_{\ell}, \dot{W}_{\ell}}-$ generic real over $\mathbf{L}[r, s]$ belonging to the set $B$.

Then there is a $\Sigma_{3}^{1}$ set which does not have $\mathcal{I}_{\mathbb{P}, \dot{W}}$-Baire property.

Proof. Since $\mathbb{Q}_{\ell}, \dot{W}_{\ell}$ are as in 3.1.1, we have a nice description of $\mathbb{Q}_{\ell}-$ names for reals, see 3.1.7. So we have Borel functions $h_{\ell}: \mathcal{X}_{\ell} \longrightarrow \mathcal{X}$ such that $\Vdash_{\mathbb{Q}_{\ell}} \dot{\tau}_{\ell}=h_{\ell}\left(\dot{W}_{\ell}\right)$. It follows from the assumed properties of $\dot{\tau}_{\ell}$ that

$(*)_{1}$ for every Borel set $A \subseteq \mathcal{X}$, if $A \notin \mathcal{I}_{\mathbb{P}, \dot{W}}$ then $h_{\ell}^{-1}[A] \notin \mathcal{I}_{\mathbb{Q}_{\ell}, \dot{W}_{\ell}}$.

(Note that $h_{\ell}$ is coded by the real $r$ as well.) Since $\omega_{1}$ is not inaccessible in $\mathbf{L}$, for some real $a$ we have $\omega_{1}^{\mathbf{L}[a]}=\omega_{1}$. Let

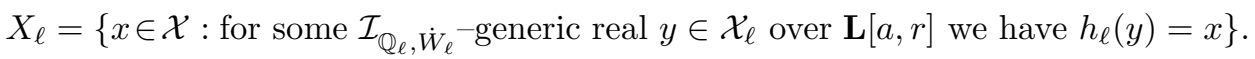

(Note that if $y$ is $\mathcal{I}_{\mathbb{Q}_{\ell}, \dot{W}_{\ell}}$-generic over $\mathbf{L}[a, r]$, then it determines a $\mathbb{Q}_{\ell}^{\mathbf{L}[a, r]}$-generic filter $G \subseteq \mathbb{Q}_{\ell}^{\mathbf{L}[a, r]}$ over $\mathbf{L}[a, r]$ and $h_{\ell}(y)=\dot{\tau}_{\ell}^{G}$; remember 3.1.5 and the choice of $h_{\ell}$.)

$\left(*_{2}\right) X_{\ell}$ is a $\Sigma_{3}^{1}$ subset of $\mathcal{X}_{\ell}$.

[Why? Note that, by 3.1.3(4), the formula " $c$ is a Borel code for a set $\sharp c \subseteq \mathcal{X}_{\ell}$ and $\sharp c \notin \mathcal{I}_{\mathbb{Q}_{\ell}, \dot{W}_{\ell}}$ " is (equivalent in ZFC to) a $\Pi_{2}^{1}$ formula. Hence easily the formula " $y$ is $\mathcal{I}_{\mathbb{Q}_{\ell}, \dot{W}_{\ell}}$-generic over $\mathbf{L}[a, r] "$ is $\Pi_{2}^{1}$. $]$

$\left(*_{3}\right)$ For every Borel set $B \subseteq \mathcal{X}$, if $B \notin \mathcal{I}_{\mathbb{P}, \dot{W}}$ then $X_{\ell} \cap B \neq \emptyset$.

[Immediate by $\left(*_{1}\right)$ and the assumption (c).]

$\left(*_{4}\right) X_{0} \cap X_{1}=\emptyset$. 
[It follows from the sourness and the assumption that $\omega_{1}^{\mathbf{L}[a]}=\omega_{1}$.]

Finally note that $\left(*_{3}\right)+\left(*_{4}\right)$ implies that both sets $X_{0}, X_{1}$ do not have the $\mathcal{I}_{\mathbb{P}, \dot{W}}$-Baire property.

Corollary 4.2.6. Suppose that $r, \mathbb{P}, \dot{W}, \mathbb{Q}_{\ell}, \dot{\tau}_{\ell}, \dot{W}_{\ell}$ are as in 4.2.5, and clauses (a)(c) there hold, and $\mathbb{Q}_{0}=\mathbb{Q}_{1}, \dot{W}_{0}=\dot{W}_{1}$. Assume additionally that, for some $k<\omega$, there are $\mathbb{Q}_{0}^{(k)}$-names $\dot{\rho}_{\ell}^{0}, \dot{\rho}_{\ell}^{1}($ for $\ell<2)$ and a $\mathbb{Q}_{0}$-name $\dot{\tau}$ such that

(d) $\Vdash_{\mathbb{Q}_{0}}$ " $\dot{\tau} \in \mathcal{X}$ is $\mathcal{I}_{\mathbb{P}, \dot{W}}$-generic over $\mathbf{V}$ ",

(e) $\Vdash_{\mathbb{Q}_{0}^{(k)}}$ " $\dot{\rho}_{\ell}^{0}, \dot{\rho}_{\ell}^{1} \in \mathcal{X}_{0}$ are $\mathcal{I}_{\mathbb{Q}_{0}, \dot{W}_{0}}$-generic over $\mathbf{V}$ ", and for $\ell<2$ and every $\mathcal{I}_{\mathbb{Q}_{0}, \dot{W}_{0}}$-positive Borel set $B \subseteq \mathcal{X}_{0}$ there is $q \in \mathbb{Q}_{0}^{(k)}$ such that $q \Vdash$ " $\dot{\rho}_{\ell}^{0} \in B$ ",

(f) $\Vdash_{\mathbb{Q}_{0}^{(k)}} " \dot{\tau}\left[\dot{\rho}_{\ell}^{0}\right]=\dot{\tau}_{\ell}\left[\dot{\rho}_{\ell}^{1}\right] "$.

(Above, $\mathbb{Q}_{0}^{(k)}$ stands for the iteration of length $k$ of the forcing notion $\mathbb{Q}_{0}$. )

Then there is a projective subset of $\mathcal{X}_{0}$ that does not have the $\mathcal{I}_{\mathbb{Q}_{0}, \dot{W}_{0}}$-Baire property.

Let us turn to getting sourness for some of the forcing notions discussed in this paper. Of course, because of 4.1.5 the forcing notions covered by 4.1 .12 are not sour (they are in the sweet kingdom, after all). However, there are sour examples around. Let us introduce them starting with exotic norm conditions which were chosen specially with the sourness in mind.

Proposition 4.2.7. Let $(K, \Sigma)$ be a local forgetful and complete (see 2.2.7) creating pair for $\mathbf{H}$.

1. Assume $(K, \Sigma)$ is linked and

$(\alpha)(\forall n<\omega)\left(|\mathbf{H}(n)|>2^{n}\right)$,

$(\beta)(\bar{K}, \bar{g})$ is a 1-norming system for $\mathbf{H}$ (see 2.2.8), $\bar{K}=\left\langle K_{\ell}: \ell<\omega\right\rangle$, $\bar{g}=\left\langle g_{\rho}: \rho \in 2^{<\omega}\right\rangle$,

$(\gamma)$ if $A \subseteq \mathbf{H}(n), a \in A$ and $\operatorname{nor}\left[t_{A}^{n}\right]>1$ then $\operatorname{nor}\left[t_{A \backslash\{a\}}^{n}\right] \geq \operatorname{nor}\left[t_{A}^{n}\right]-1$,

$(\delta)$ letting $A_{n}=\mathbf{H}(n) \backslash\left\{g_{\rho}(n): \rho \in 2^{\ell}\right\}$ for $n \in K_{\ell}$, and $A_{n}=\mathbf{H}(n)$ for $n \notin \bigcup_{\ell<\omega} K_{\ell}$, we have $\lim _{n \rightarrow \infty} \operatorname{nor}\left[t_{A_{n}}^{n}\right]=\infty$.

(Above, for $n \in \omega$ and $A \subseteq \mathbf{H}(n), t_{A}^{n}$ is the unique creature $t \in K$ with $m_{\mathrm{dn}}^{t}=n$ and $\operatorname{pos}(t)=A$; see 2.2.7.)

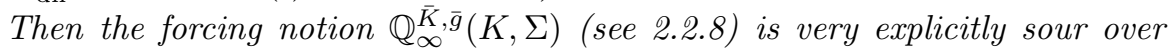
Cohen.

2. If $(K, \Sigma),(\bar{K}, \bar{g})$ and $\mathbf{H}$ are as in (1), and $f: \omega \times \omega \longrightarrow \omega$ is fast and

$$
(\forall n<\omega)(\forall i \geq n)\left(f(n, i) \leq \operatorname{nor}\left[t_{\mathbf{H}(i)}^{i}\right]\right),
$$

then $\mathbb{Q}_{f}^{\bar{K}, \bar{g}}(K, \Sigma)$ is very explicitly sour over Cohen.

3. Assume that $h: \omega \times \omega \longrightarrow \omega$ is regressive, $\mathcal{F} \subseteq(\omega \backslash 2)^{\omega}$ is $h$-closed, countable and $\geq^{*}$-directed. Suppose that $(K, \Sigma)$ is $h$-linked and clauses $(\alpha)-(\gamma)$ hold true, and

$\left(\delta^{+}\right)$for $A_{n}$ as in $(\delta)$, for each $f \in \mathcal{F}$ we have $\left(\forall^{\infty} n \in \omega\right)\left(f(n) \leq \operatorname{nor}\left[t_{A_{n}}^{n}\right]\right)$.

Then the forcing notion $\mathbb{Q}_{\mathcal{F}}^{\bar{K}}, \bar{g}(K, \Sigma)$ is very explicitly sour over Cohen.

4. Assume that $(K, \Sigma)$ is linked, satisfies the demand $1(\gamma)$, and $\lim _{n \rightarrow \infty} \operatorname{nor}\left[t_{\mathbf{H}(n)}^{n}\right]=$ $\infty$. Let $\bar{U}=\left\langle U_{\rho, k}: \rho \in 2^{<\omega} \& k<\omega\right\rangle$ be a 2-norming system (see 2.2.10). Then the forcing notion $\mathbb{Q}_{\infty}^{\bar{U}}(K, \Sigma)$ (see 2.2.10) is very explicitly sour over Cohen. 
5. Similarly for forcing notions $\mathbb{Q}_{f}^{\bar{U}}(K, \Sigma), \mathbb{Q}_{\mathcal{F}}^{\bar{U}}(K, \Sigma)$ (under assumptions parallel to that in 2,3 above, with clause $\left(\delta^{+}\right)$replaced by just $f(n) \leq \operatorname{nor}\left[t_{\mathbf{H}(n)}^{n}\right]$ for $n<\omega, f \in \mathcal{F})$.

Proof. 1) Let us think about the Cohen forcing $\mathbb{C}$ as the set of partial functions from $2^{<\omega}$ to 2 with the relation of extension; $\mathbf{H}_{\mathbb{C}}(\rho)=2$ for $\rho \in 2^{<\omega}$ (so we interpret $\omega$ as $2^{<\omega}$ ), and $\dot{W}_{\mathbb{C}}$ is the natural name for the $\mathbb{C}$-generic real in $2^{2^{<\omega}}$.

Let $\dot{W}$ be the name for $\mathbb{Q}_{\infty}^{\bar{K}, \bar{g}}(K, \Sigma)$-generic real, i.e., $\Vdash \dot{W}=\bigcup\left\{w^{q}: q \in\right.$ $\left.\Gamma_{\mathbb{Q}_{\infty}^{\bar{x}, \bar{g}}(K, \Sigma)}\right\}$. Let $\dot{\tau}_{0}, \dot{\tau}_{1}$ be standard $\mathbb{Q}_{\infty}^{\bar{K}, \bar{g}}(K, \Sigma)$-names for functions from $2^{<\omega}$ to 2 such that

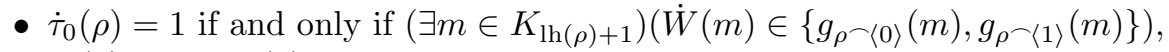

- $\dot{\tau}_{1}(\rho)=1-\dot{\tau}_{0}(\rho)$.

Claim 4.2.7.1.

$$
\Vdash_{\mathbb{Q}_{\infty}^{\bar{K}, \bar{g}}(K, \Sigma)} \text { “ } \dot{\tau}_{0}, \dot{\tau}_{1} \text { are } \mathbb{C} \text {-generic over } \mathbf{V} \text { "; }
$$

moreover $\llbracket \dot{\tau}_{\ell} \in B \rrbracket_{\mathbb{Q}_{\infty}^{\bar{K}, \bar{g}}(K, \Sigma)} \neq \mathbf{0}$ for any non-meager Borel set $B \subseteq 2^{2^{<\omega}}$. Hence $\left(\mathbb{C}, \dot{W}_{\mathbb{C}}\right),\left(\mathbb{Q}_{\infty}^{\bar{K}, \bar{g}}(K, \Sigma), \dot{\tau}_{0}\right),\left(\mathbb{Q}_{\infty}^{\bar{K}, \bar{g}}(K, \Sigma), \dot{\tau}_{1}\right)$ are as in 4.2.1.

Proof of the claim. Let $q \in \mathbb{Q}_{\infty}^{\bar{K}, \bar{g}}(K, \Sigma)$ and let $\rho_{0}, \ldots, \rho_{k} \in 2^{\omega}$ be such that

$$
\rho \in 2^{\ell} \& m_{\mathrm{dn}}^{t_{i}^{q}} \in K_{\ell} \& g_{\rho}\left(m_{\mathrm{dn}}^{t_{i}^{q}}\right) \notin \operatorname{pos}\left(t_{i}^{q}\right) \quad \Rightarrow \quad \rho \triangleleft \rho_{0} \vee \ldots \vee \rho \triangleleft \rho_{k} .
$$

Pick $N \geq \operatorname{lh}\left(w^{q}\right)$ such that $\rho_{0}\left\lceil N, \ldots, \rho_{k}\lceil N\right.$ are pairwise distinct and

(a) $m_{\mathrm{dn}}^{t_{i}^{q}} \geq N \Rightarrow \operatorname{nor}\left[t_{i}^{q}\right] \geq 2$, and

(b) $n \geq N \Rightarrow \operatorname{nor}\left[t_{A_{n}}^{n}\right] \geq 2$, where $A_{n}$ is as in the assumption $(\delta)$.

Suppose $M>N$ and $h:\left\{\rho \in 2^{<\omega}: N<\operatorname{lh}(\rho) \leq M\right\} \longrightarrow 2$. Build a condition $p=\left(w^{p}, t_{0}^{p}, t_{1}^{p}, \ldots\right)$ such that

(c) $w^{q} \triangleleft w^{p}, w^{p}(m) \in \operatorname{pos}\left(t_{j}^{q}\right)$ if $m=m_{\mathrm{dn}}^{t_{j}^{q}}<\operatorname{lh}\left(w^{p}\right)$, and $t_{i}^{p} \in \Sigma\left(t_{j}^{q}\right)$ whenever $m_{\mathrm{dn}}^{t_{j}^{q}}=m_{\mathrm{dn}}^{t_{i}^{p}}$

(d) if $N<\operatorname{lh}(\rho) \leq M, h(\rho)=1$, then for some $m=m(\rho) \in K_{\operatorname{lh}(\rho)+1}$ we have

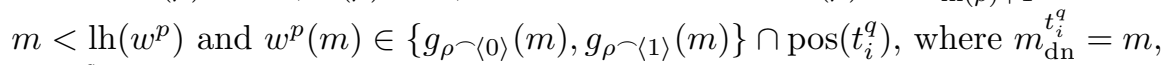

(e) if $m_{\mathrm{dn}}^{t_{i}^{q}} \geq \operatorname{lh}\left(w^{p}\right)$ then $\operatorname{nor}\left[t_{i}^{q}\right] \geq 2^{M+3}$,

(f) if $m<\operatorname{lh}\left(w^{p}\right)$ is not any of the $m(\rho)$ 's from clause (d) above (for $h(\rho)=1$ ), $m \in K_{\ell}, \ell>N$, then $w^{p}(m) \in \operatorname{pos}\left(t_{i}^{q}\right) \backslash\left\{g_{\rho}(m): \rho \in 2^{\ell}\right\}$, where $m_{\mathrm{dn}}^{t_{i}^{q}}=m$ (remember: $(K, \Sigma)$ is linked and (a) $+(\mathrm{b}))$,

(g) if $m=\ln \left(w^{p}\right)+i \in K_{\ell}, N<\ell \leq M+1$ and $m_{\mathrm{dn}}^{t_{j}^{q}}=m$, then $t_{i}^{p} \in \Sigma\left(t_{j}^{q}\right)$ is such that

$$
\operatorname{nor}\left[t_{i}^{p}\right] \geq \operatorname{nor}\left[t_{j}^{q}\right]-2^{M+1} \quad \text { and } \quad \operatorname{pos}\left(t_{i}^{p}\right) \cap\left\{g_{\rho}(m): \rho \in 2^{\ell}\right\}=\emptyset
$$

(remember assumption $(\gamma)$ and clause $(\mathrm{e})$ ),

(h) if $m=\ln \left(w^{p}\right)+i \notin \bigcup_{\ell=N+1}^{M} K_{\ell}, m=m_{\mathrm{dn}}^{t_{j}^{q}}$, then $t_{i}^{p}=t_{j}^{q}$.

It should be clear that we can build $p \in \mathbb{Q}_{\infty}^{\bar{K}, \bar{g}}(K, \Sigma)$ satisfying the demands (c)-(h) and that then $q \leq p$ and $p \Vdash h \subseteq \dot{\tau}_{0}$.

Now we easily conclude that $\dot{\tau}_{0}$ is Cohen over $\mathbf{V}$; the rest should be clear too. 
For $\rho \in 2^{\omega}$ and $k<\omega$ let $p_{\rho, k} \in \mathbb{Q}_{\infty}^{\bar{K}, \bar{g}}(K, \Sigma)$ be such that $w^{p_{\rho, k}}=\langle\rangle$, if $i \in K_{\ell}$, $i>k$, then $\operatorname{pos}\left(t_{i}^{p_{\rho, k}}\right)=\mathbf{H}(i) \backslash\left\{g_{\rho \mid \ell}(i)\right\}$, and if either $i \leq k$ or $i \notin \bigcup_{\ell \in \omega} K_{\ell}$ then $\operatorname{pos}\left(t_{i}^{p_{\rho, k}}\right)=\mathbf{H}(i)$. Note that the function $g^{*}: 2^{\omega} \times \omega \longrightarrow \mathbb{Q}_{\infty}^{\bar{K}, \bar{g}}(K, \Sigma):(\rho, k) \mapsto p_{\rho, k}$ is Borel.

Claim 4.2.7.2. 1. For each $\rho \in 2^{\omega}$ the set $\left\{p_{\rho, k}: k<\omega\right\}$ is predense in $\mathbb{Q}_{\infty}^{\bar{K}, \bar{g}}(K, \Sigma)$.

2. If $\rho_{0}, \rho_{1} \in 2^{\omega}, \rho_{0}\left\lceil m=\rho_{1}\left\lceil m=\sigma, \rho_{0}(m)=0, \rho_{1}(m)=1\right.\right.$ and $k<m$, then

$$
\left(\forall q \in \mathbb{Q}_{\infty}^{\bar{K}, \bar{g}}(K, \Sigma)\right)\left(q \geq p_{\rho_{0}, k} \& q \geq p_{\rho_{1}, k} \quad \Rightarrow \quad q \Vdash \dot{\tau}_{0}(\sigma)=0\right) .
$$

Proof of the claim. (1) Straightforward.

(2) Note that if $q \geq p_{\rho_{0}, k}, q \geq p_{\rho_{1}, k}$ then for each $i, n=m_{\mathrm{dn}}^{t_{i}^{q}} \in K_{m+1}$ implies $\left(n \geq m+1>k\right.$ and) $g_{\rho_{0} \uparrow(m+1)}(n), g_{\rho_{1} \uparrow(m+1)}(n) \notin \operatorname{pos}\left(t_{i}^{q}\right)$ (and, of course, $\sigma \prec\langle 0\rangle=$ $\left.\rho_{0} \uparrow(m+1), \sigma \curlyvee\langle 1\rangle=\rho_{1} \uparrow(m+1)\right)$. Also, if $n<\operatorname{lh}\left(w^{q}\right), n \in K_{m+1}$, then $(n>k$ and $)$ $w^{q}(n) \notin\left\{g_{\rho_{0} \uparrow(m+1)}(n), g_{\rho_{1} \uparrow(m+1)}\right\}$.

Now one easily shows that $\left(\mathbb{Q}_{\infty}^{\bar{K}, \bar{g}}(K, \Sigma), \dot{\tau}_{0}\right)$ is very explicitly sour to $\left(\mathbb{Q}_{\infty}^{\bar{K}, \bar{g}}(K, \Sigma), \dot{\tau}_{1}\right)$.

2), 3) Similarly.

4) Here we think about the Cohen forcing $\mathbb{C}$ as the set of finite partial functions from $2^{<\omega} \times \omega$ to 2 ordered by the extension; $\mathbf{H}_{\mathbb{C}}, \dot{W}_{\mathbb{C}}$ are interpreted suitably.

For each $n<\omega$ pick $a_{n} \in \mathbf{H}(n)$. Let $\dot{W}$ be the name for $\mathbb{Q}_{\infty}^{\bar{U}}(K, \Sigma)$-generic real, and take standard $\mathbb{Q}_{\infty}^{\bar{U}}(K, \Sigma)$-names $\dot{\tau}_{0}, \dot{\tau}_{1}$ for functions from $2^{<\omega} \times \omega$ from 2 such that

- $\dot{\tau}_{0}(\sigma, k)=0$ if and only if $\left(\forall n \in U_{\sigma \frown\langle 0\rangle, k} \cup U_{\sigma \frown\langle 1\rangle, k}\right)\left(\dot{W}(n) \neq a_{n}\right)$,

- $\dot{\tau}_{1}(\sigma, k)=1-\dot{\tau}_{0}(\sigma, k)$.

Claim 4.2.7.3.

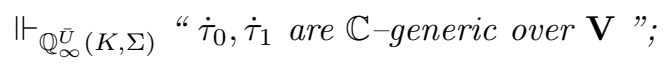

moreover $\llbracket \dot{\tau}_{\ell} \in B \rrbracket_{\mathbb{Q}_{\infty}^{\bar{U}}(K, \Sigma)} \neq \mathbf{0}$ for any non-meager Borel set $B \subseteq 2^{2^{<\omega}}$. Hence $\left(\mathbb{C}, \dot{W}_{\mathbb{C}}\right),\left(\mathbb{Q}_{\infty}^{\bar{U}}(K, \Sigma), \dot{\tau}_{0}\right),\left(\mathbb{Q}_{\infty}^{\bar{U}}(K, \Sigma), \dot{\tau}_{1}\right)$ are as in 4.2.1.

Proof of the claim. Quite similar to 4.2.7.1.

Now, for $\rho \in 2^{\omega}$ and $n, k<\omega$ let $p_{\rho, k}^{n} \in \mathbb{Q}_{\infty}^{\bar{U}}(K, \Sigma)$ be such that $w^{p_{\rho, k}^{n}}=\langle\rangle$, and if $i \in U_{\rho\lceil m, k}, m<\omega, n \leq i$, then $\operatorname{pos}\left(t_{i}^{p_{\rho, k}^{n}}\right)=\mathbf{H}(i) \backslash\left\{a_{i}\right\}$, and $\operatorname{pos}\left(t_{i}^{p_{\rho}^{n}, k}\right)=\mathbf{H}(i)$ is all other cases.

Claim 4.2.7.4. 1. For each $\rho \in 2^{\omega}$ and $k \in \omega$, the set $\left\{p_{\rho, k}^{n}: n<\omega\right\}$ is predense in $\mathbb{Q}_{\infty}^{\bar{U}}(K, \Sigma)$.

2. If $k<\omega, \rho_{0}, \rho_{1} \in 2^{\omega}, \rho_{0}\left\lceil m=\rho_{1}\left\lceil m=\sigma, \rho_{0}(m) \neq \rho_{1}(m)\right.\right.$ and $n<m$, then

$$
\left(\forall q \in \mathbb{Q}_{\infty}^{\bar{U}}(K, \Sigma)\right)\left(q \geq p_{\rho_{0}, k}^{n} \& q \geq p_{\rho_{1}, k}^{n} \quad \Rightarrow \quad q \Vdash \dot{\tau}_{0}(\sigma, k)=0\right) .
$$

Proof of the claim. Straightforward.

Now we easily finish.

5) Similarly. 
Definition 4.2.8. Let $(K, \Sigma)$ be a local creating pair for $\mathbf{H}$ and let $\mathcal{F} \subseteq(\omega \backslash 2)^{\omega}$. $A$ sourness system for $(K, \Sigma, \mathcal{F})$ is a pair $(\bar{g}, \bar{\ell})$ such that

$(\alpha) \bar{\ell}=\left\langle\ell_{k}: k<\omega\right\rangle \subseteq \omega$ is increasing,

( $\beta) \bar{g}=\left\langle g_{\rho}: \rho \in 2^{<\bar{\omega}}\right\rangle$, if $\rho \in 2^{k}$ then $g_{\rho} \in \prod_{i<\ell_{k}} \mathcal{P}(\mathbf{H}(i))$, and $\rho \triangleleft \rho^{\prime} \Rightarrow g_{\rho} \triangleleft g_{\rho^{\prime}}$,

$(\gamma)$ for each $n \in\left[\ell_{k}, \ell_{k+1}\right), k<\omega$, the sets $\left\{g_{\rho}(n): \rho \in 2^{k+1}\right\}$ are pairwise disjoint and non-empty, and $\mathbf{H}(n) \backslash \bigcup\left\{g_{\rho}: \rho \in 2^{k+1}\right\} \neq \emptyset$,

$(\delta)$ if $f \in \mathcal{F},\left\langle t_{0}, t_{1}, \ldots\right\rangle \in \mathrm{PC}(K, \Sigma)$ (see 1.1.9), $m_{\mathrm{dn}}^{t_{0}}=\ell_{k_{0}}, \operatorname{nor}\left[t_{n}\right] \geq f\left(n+\ell_{k_{0}}\right)$ for $n<\omega$ then:

(i) for some $N<\omega$, for each $k \geq k_{0}$, we have

$\left|\left\{\rho \in 2^{k+1}:\left|\left\{n \in\left[\ell_{k}, \ell_{k+1}\right): g_{\rho}(n) \cap \operatorname{pos}\left(t_{n-\ell_{k_{0}}}\right) \neq \emptyset\right\}\right|<2^{k+1}\right\}\right| \leq N$,

(ii) for some $k_{1} \geq k_{0}$ we have

$$
\left(\forall k \geq k_{1}\right)\left(\forall n \in\left[\ell_{k}, \ell_{k+1}\right)\right)\left(\operatorname{pos}\left(t_{n-\ell_{k_{0}}}\right) \backslash \bigcup\left\{g_{\rho}(n): \rho \in 2^{k+1}\right\} \neq \emptyset\right)
$$

Theorem 4.2.9. Suppose that $h: \omega \times \omega \longrightarrow \omega$ is regressive, and $\mathcal{F} \subseteq(\omega \backslash 2)^{\omega}$ is a countable $h$-closed and $\geq^{*}$-directed family. Let $(K, \Sigma)$ be a local, $h$-linked and complete creating pair for $\mathbf{H}$ such that

(a) for some $f^{*} \in \mathcal{F}$ we have that $(\forall n<\omega)\left(\operatorname{nor}\left[t_{\mathbf{H}(n)}^{n}\right] \geq f^{*}(n)\right)$ (see 2.2. $)$,

(b) there is a sourness system $(\bar{g}, \bar{\ell})$ for $(K, \Sigma, \mathcal{F})$; let $\bar{g}=\left\langle g_{\rho}: \rho \in 2^{<\omega}\right\rangle$, $\bar{\ell}=\left\langle\ell_{k}: k<\omega\right\rangle$,

(c) if $A \subseteq \mathbf{H}(n), \ell_{k} \leq n<\ell_{k+1}, \rho \in 2^{k+1}$ and $A \backslash g_{\rho}(n) \neq \emptyset$, then

$$
\operatorname{nor}\left[t_{A \backslash g_{\rho}(n)}^{n}\right] \geq h\left(n, \operatorname{nor}\left[t_{A}^{n}\right]\right) .
$$

Then the forcing notion $\mathbb{Q}_{\mathcal{F}}^{*}(K, \Sigma)$ is very explicitly sour over Cohen.

Proof. Here we interpret the Cohen forcing notion $\mathbb{C}$ in the standard way, i.e., it is $\left(2^{<\omega}, \triangleleft\right)$ (and $\mathbf{H}_{\mathbb{C}}, \dot{W}_{\mathbb{C}}$ are natural).

For $\rho \in 2^{\omega}$ let $g_{\rho}=\bigcup_{i \in \omega} g_{\rho \uparrow i} \in \prod_{i \in \omega} \mathcal{P}(\mathbf{H}(i))$ (remember $1.2 .8(\beta)$ ). Let $\dot{W}$ be the canonical name for the $\mathbb{Q}_{\mathcal{F}}^{*}(K, \Sigma)$-generic real and let $\dot{\tau}_{0}, \dot{\tau}_{1}$ be standard $\mathbb{Q}_{\mathcal{F}}^{*}(K, \Sigma)$ names for reals in $2^{\omega}$ such that

- $\dot{\tau}_{0}(k)=0$ if and only if there are $\rho_{0}, \rho_{1} \in 2^{\omega}$ such that $\rho_{0}\left\lceil k=\rho_{1}\lceil k\right.$, $\rho_{0}(k) \neq \rho_{1}(k)$ and $\left(\forall n \geq \ell_{k}\right)\left(\dot{W}(n) \notin g_{\rho_{0}}(n) \cup g_{\rho_{1}}(n)\right)$;

- $\dot{\tau}_{1}(n)=1-\dot{\tau}_{0}(n)$.

Claim 4.2.9.1. $\left(\mathbb{C}, \dot{W}_{\mathbb{C}}\right),\left(\mathbb{Q}_{\mathcal{F}}^{*}(K, \Sigma), \dot{\tau}_{0}\right),\left(\mathbb{Q}_{\mathcal{F}}^{*}(K, \Sigma), \dot{\tau}_{1}\right)$ are as in 4.2 .1 .

Proof of the claim. We will show that $\dot{\tau}_{0}$ is (a name for) a Cohen real over $\mathbf{V}$; then the rest should be clear.

So suppose $p=\left(w^{p}, t_{0}^{p}, t_{1}^{p}, \ldots\right) \in \mathbb{Q}_{\mathcal{F}}^{*}(K, \Sigma)$ and we may assume that $\ln \left(w^{p}\right)=$ $\ell_{k_{0}}, k_{0}<\omega$. For $k \geq k_{0}$ let

$$
\Upsilon_{k}^{p}=\Upsilon_{k} \stackrel{\text { def }}{=}\left\{\rho \in 2^{k+1}:\left|\left\{n \in\left[\ell_{k}, \ell_{k+1}\right): g_{\rho}(n) \cap \operatorname{pos}\left(t_{n-\ell_{k_{0}}}^{p}\right) \neq \emptyset\right\}\right|<2^{k+1}\right\},
$$

and let $N$ be such that $\left(\forall k \geq k_{0}\right)\left(\left|\Upsilon_{k}\right|<N\right)$ (remember 4.2.8( $(\delta(\mathrm{i}))$. Let

$$
u_{k}=\left\{i \leq k:\left(\exists \rho_{0}, \rho_{1} \in \Upsilon_{k}\right)\left(\rho_{0}\left\lceil i=\rho_{1}\left\lceil i \& \rho_{0}(i) \neq \rho_{1}(i)\right)\right\} .\right.\right.
$$


Clearly, for all $k \geq k_{0},\left|u_{k}\right| \leq N$, and thus we may choose an infinite set $A \subseteq \omega$ such that $\left\{u_{k}: k \in A\right\}$ forms a $\Delta$-system with the heart, say, $u^{*}$. Take $k_{1}>$ $\max \left(u^{*}\right)+k_{0}+N$ such that

$\left(*_{1}\right)\left(\forall k \geq k_{1}\right)\left(\forall n \in\left[\ell_{k}, \ell_{k+1}\right)\right)\left(\operatorname{pos}\left(t_{n-\ell_{k_{0}}}^{p}\right) \backslash \bigcup\left\{g_{\rho}(n): \rho \in 2^{k+1}\right\} \neq \emptyset\right)$

(remember $4.2 .8(\delta(\mathrm{ii}))$ and suppose that $v \subseteq\left[k_{1}, k_{2}\right), k_{2}>k_{1}$. We are going to build a condition $q \geq p$ such that

$$
q \Vdash\left(\forall j \in\left[k_{1}, k_{2}\right)\right)\left(\dot{\tau}_{0}(j)=0 \Leftrightarrow j \in v\right) .
$$

To this end, pick $i>k_{2}$ such that

$\left(*_{2}\right) u_{i} \cap\left[k_{1}, k_{2}\right)=\emptyset$, and

$\left(*_{3}\right)$ for some $f \in \mathcal{F}$ we have

$$
\left(\forall n \geq \ell_{i+1}\right)\left(f(n)<h^{\left(k_{2}\right)}\left(n, \operatorname{nor}\left[t_{n-\ell_{k_{0}}}^{p}\right]\right),\right.
$$

where $h^{(k+1)}(n, m)=h\left(n, h^{(k)}(n, m)\right)$.

(Possible by the choice of $k_{1}$ and the assumption that $\mathcal{F}$ is $h$-closed.) Since $\left|\Upsilon_{i}\right|<$ $N<2^{k_{1}}$, we may find $\rho^{*} \in 2^{\omega}$ such that $\rho^{*}\left\lceil k_{1} \notin\left\{\sigma\left\lceil k_{1}: \sigma \in \Upsilon_{i}\right\}\right.\right.$. For $k \in v$ fix $\rho_{k} \in 2^{\omega}$ such that $\rho_{k}\left\lceil k=\rho^{*}\left\lceil k, \rho_{k}(k)=1-\rho^{*}(k)\right.\right.$.

Let $\left\langle\sigma_{j}: j<2^{i+1}-|v|-1\right\rangle$ enumerate $2^{i+1} \backslash\left\{\rho^{*} \uparrow(i+1), \rho_{k} \uparrow(i+1): k \in v\right\}$. By induction on $j<2^{i+1}-|v|-1$ define $n_{j}^{*} \in\left[\ell_{i}, \ell_{i+1}\right) \cup\{*\}$ as follows:

if there is $n \in\left[\ell_{i}, \ell_{i+1}\right) \backslash\left\{n_{j^{\prime}}^{*}: j^{\prime}<j\right\}$ such that $g_{\sigma_{j}}(n) \cap \operatorname{pos}\left(t_{n-\ell_{k_{0}}}^{p}\right) \neq \emptyset$, then $n_{j}^{*}$ is the first such number, otherwise $n_{j}^{*}$ is *.

Now we choose $w^{q}, t_{0}^{q}, t_{1}^{q}, \ldots$ so that:

(i) $\operatorname{lh}\left(w^{q}\right)=\ell_{i+1}, w^{p} \triangleleft w^{q}$;

(ii) if $n \in\left[\ell_{i}, \ell_{i+1}\right), n=n_{j}^{*}, j<2^{i+1}-|v|-1$, then $w^{q}(n) \in g_{\sigma_{j}}(n) \cap \operatorname{pos}\left(t_{n-\ell_{k_{0}}}^{p}\right)$;

(iii) if $\ell_{k_{0}} \leq n<\ell_{k_{1}}$, then $w^{q}(n) \in \operatorname{pos}\left(t_{n-\ell_{k_{0}}}^{p}\right)$;

(iv) if $n \in\left[\ell_{k_{1}}, \ell_{i}\right) \cup\left(\left[\ell_{i}, \ell_{i+1}\right) \backslash\left\{n_{j}^{*}: j<2^{i+1}-|v|-1\right\}\right)$, then

$$
w^{q}(n) \in \operatorname{pos}\left(t_{n-\ell_{k_{0}}}^{p}\right) \backslash \bigcup\left\{g_{\rho}(n): \rho \in 2^{i+1}\right\} ;
$$

(v) $t_{n}^{q} \in \Sigma\left(t_{n+\ell_{i+1}-\ell_{k_{0}}}^{p}\right)$ is such that $\operatorname{nor}\left[t_{n}^{q}\right] \geq f\left(n+\ell_{i+1}\right)$ and

$$
\left(g_{\rho^{*}}\left(n+\ell_{i+1}\right) \cup \bigcup_{k \in v} g_{\rho_{k}}\left(n+\ell_{i+1}\right)\right) \cap \operatorname{pos}\left(t_{n}^{q}\right)=\emptyset
$$

(where $f$ is given by $\left(*_{3}\right)$ ).

[Why is the choice possible? Demands (i)-(iii) are easy; (iv) can be satisfied by $\left(*_{1}\right)$, remember $4.2 .8(\beta) ;(\mathrm{v})$ is possible by the assumption $(\mathrm{c})$ of the theorem and $\left(*_{3}\right)$.] One easily checks that the demands (i) $-(\mathrm{v})$ imply $q=\left(w^{q}, t_{0}^{q}, t_{1}^{q}, \ldots\right)$ is a condition in $\mathbb{Q}_{\mathcal{F}}^{*}(K, \Sigma)$ stronger than $p$. Also, by (ii) $+(\mathrm{iv})+(\mathrm{v})$,

$$
q \Vdash(\forall k \in v)\left(\forall n \geq \ell_{k}\right)\left(\dot{W}(n) \notin g_{\rho^{*}}(n) \cup g_{\rho_{k}}(n)\right)
$$

(remember 4.2.8 $(\gamma)$; thus $g_{\sigma_{j}}(n) \cap g_{\rho^{*}}(n)=g_{\sigma_{j}}(n) \cap g_{\rho_{k}}(n)=\emptyset$ in clause (ii)). Hence $q \Vdash(\forall k \in v)\left(\dot{\tau}_{0}(k)=0\right)$. Now we argue that $q \Vdash\left(\forall k \in\left[k_{1}, k_{2}\right) \backslash v\right)\left(\dot{\tau}_{0}(k)=1\right)$. If not, then for some $k \in\left[k_{1}, k_{2}\right) \backslash v$ we find $\rho_{0}^{+}, \rho_{1}^{+} \in 2^{i+1}$ such that $\rho_{0}^{+}\left\lceil k=\rho_{1}^{+}\left\lceil k, \quad \rho_{0}^{+}(k) \neq \rho_{1}^{+}(k), \quad\right.\right.$ and $\quad\left(\forall n \in\left[\ell_{k}, \ell_{i+1}\right)\right)\left(w^{q}(n) \notin g_{\rho_{0}^{+}}(n) \cup g_{\rho_{1}^{+}}(n)\right)$. 
Necessarily, $\left\{\rho_{0}^{+}, \rho_{1}^{+}\right\} \nsubseteq\left\{\rho^{*} \uparrow(i+1), \rho_{k} \uparrow(i+1): k \in v\right\}$. Moreover, if $\rho_{\ell}^{+} \in\left\{\sigma_{j}:\right.$ $\left.j<2^{i+1}-|v|-1\right\}$, then $\rho_{\ell}^{+} \in \Upsilon_{i}$ (as if $\rho_{\ell}^{+}=\sigma_{j} \notin \Upsilon_{i}$ then $n_{j}^{*} \in\left[\ell_{i}, \ell_{i+1}\right)$ and $\left.w^{q}\left(n_{j}^{*}\right) \in g_{\rho_{\ell}^{+}}\left(n_{j}^{*}\right)\right)$. Since $\rho^{*}\left\lceil k_{1} \notin\left\{\sigma\left\lceil k_{1}: \sigma \in \Upsilon_{i}\right\}\right.\right.$, we may conclude that $\rho_{0}^{+}, \rho_{1}^{+} \in\left\{\sigma_{j}: j<2^{i+1}-|v|-1\right\}$ (and thus both are in $\Upsilon_{i}$ ). However, then we get $k \in u_{i}$, what contradicts $\left(*_{2}\right)$.

For $\rho \in 2^{\omega}$ and $n \in \omega$ let $p_{\rho, n} \in \mathbb{Q}_{\mathcal{F}}^{*}(K, \Sigma)$ be such that $w^{p_{\rho, n}}=\langle\rangle, t_{k}^{p_{\rho, n}}=t_{\mathbf{H}(k)}^{k}$ for $k<n$, and $t_{k}^{p_{\rho, n}}=t_{\mathbf{H}(k) \backslash g_{\rho}(k)}^{k}$ for $k \geq n$. Let

$$
g_{0}, g_{1}: 2^{\omega} \times \omega \longrightarrow \mathbb{Q}_{\mathcal{F}}^{*}(K, \Sigma):(\rho, n) \mapsto p_{\rho, n}
$$

It is straightforward to check that $g_{0}, g_{1}$ witness $\left(\mathbb{Q}_{\mathcal{F}}^{*}(K, \Sigma), \dot{\tau}_{0}\right)$ is very explicitly sour to $\left(\mathbb{Q}_{\mathcal{F}}^{*}(K, \Sigma), \dot{\tau}_{1}\right)$ (note that if $k, k^{\prime}<m, \rho_{0}, \rho_{1} \in 2^{\omega}, \rho_{0}\left\lceil m=\rho_{1}\left\lceil m, \rho_{0}(m) \neq\right.\right.$ $\rho_{1}(m)$ and $q \geq g_{0}\left(\rho_{0}, k\right), g_{1}\left(\rho_{1}, k^{\prime}\right)$, then $q \Vdash " \dot{\tau}_{0}(m)=0 \& \dot{\tau}_{1}(m)=1$ ").

Remark 4.2.10. In 4.2.9, in the assumptions on the family $\mathcal{F}$, instead of demanding that " $\mathcal{F}$ is countable $h$-closed and $\geq^{*}$-directed", we may require that " $\mathcal{F}$ is $h^{-}$ closed and either countable or $\geq^{*}$-directed", and then conclude that $\mathbb{Q}_{\mathcal{F}}^{*}(K, \Sigma)$ is explicitly sour. The "countable and $\geq^{*}$-directed" assumption is needed only to be in the context of 4.2 .1 (i.e., to make sure that $\mathbb{Q}_{\mathcal{F}}^{*}(K, \Sigma)$ is Souslin).

\subsection{Conclusions.}

Conclusion 4.3.1. 1. Let $\mathbf{H}: \omega \longrightarrow \mathcal{H}\left(\omega_{1}\right)$. The following forcing notions are topologically sweet:

(a) $\mathbb{Q}_{\infty}^{*}\left(K_{\mathbf{H}}, \Sigma_{\mathbf{H}}, \Sigma_{\mathbf{H}}^{\perp}\right)$ of 1.5 .3 and $\mathbb{Q}_{\infty}^{*}\left(K \overline{1.5 .5}, 2 \sqrt{1.5 .5}, 2 \Sigma_{1.5 .5}\right)$,

(b) $\mathbb{Q}_{f}^{*}\left(K \sqrt{1.5 .7}, \Sigma_{1.5 .7}\right)$ for $f$ as in $1.5 .8(1)$, and $\mathbb{Q}_{\mathcal{F}}^{*}\left(K \frac{1.5 .7}{1.5 .7}\right)$ for $\mathcal{F}$ as defined in 1.5.8(2) (the "e.g." part).

Conclusion 4.3.2. The forcing notions $\mathbb{Q}^{\text {tree }}(\mathfrak{p})$ for the universal parameters $\mathfrak{p}$ defined in 2.4.7, 2.4.9 and 2.4.10 are iterably sweet.

Conclusion 4.3.3. Let $\mathbf{H}: \omega \longrightarrow \omega, \mathbf{H}(i) \geq 2^{2^{i+1}}$, and let $h$ be as in 1.5.9.

1. Let $f_{k}^{0}(n)=\max \left\{2, \mathbf{H}(n)-2^{k n}\right\}$ and $\mathcal{F}_{0}=\left\{f_{k}^{0}: k<\omega\right\}$. Then the forc-

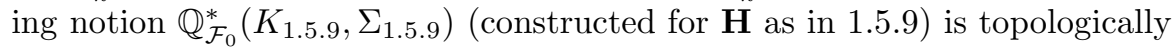
sweet.

2. Let $f_{k}^{1}(n)=\max \left\{2, \mathbf{H}(n)-2^{k}\right\}, \mathcal{F}_{1}=\left\{f_{k}^{1}: k<\omega\right\}$. Then the forcing notion $\mathbb{Q}_{\mathcal{F}_{1}}^{*}(K$ 1.5.9, 2 1.5.9) is very explicitly sour over Cohen.

Similarly if $f_{k}^{2}(n)=\max \left\{2, \mathbf{H}(n)-\left(k 2^{n}\right)\right\}, \mathcal{F}_{2}=\left\{f_{k}^{2}: k<\omega\right\}$.

Proof. (2) We are going to apply 4.2.9. First note that even though $(K \overline{1.5 .9}, 2 \sqrt{1.5 .9})$ as defined in 1.5 .9 is not complete we can easily make it so, or restrict our attention to the forcing notion below some condition (the problems with completeness come from the technical requirement in the definition of $t \in K \overline{1.5 .9}$ that $E_{t} \neq \emptyset$ ).

We are going to build a sourness system $(\bar{g}, \bar{\ell})$ for $\left(K \overline{1.5 .9}, 2 \sqrt{1.5 .9}, \mathcal{F}_{1}\right)$ such that the demand 4.2.9 (c) holds.

Let $\ell_{0}=0, \ell_{k+1}=\ell_{k}+2^{2^{k}}$. For $\rho \in 2^{<\omega}$ pick $g_{\rho}$ such that

$\left(\oplus_{1}\right)$ if $\rho \in 2^{k}$, then $g_{\rho} \in \prod_{i<\ell_{k}} \mathbf{H}(i)$, and $\rho \triangleleft \rho^{\prime} \Rightarrow g_{\rho} \triangleleft g_{\rho^{\prime}}$,

$\left(\oplus_{2}\right)$ if $n \in\left[\ell_{k}, \ell_{k+1}\right), k<\omega$, then there are no repetitions in the sequence $\left\langle g_{\rho}(n)\right.$ : $\left.\rho \in 2^{k+1}\right\rangle$. 
We claim that, letting $\bar{\ell}=\left\langle\ell_{k}: k<\omega\right\rangle$ and $\bar{g}=\left\langle g_{\rho}: \rho \in 2^{<\omega}\right\rangle,(\bar{g}, \bar{\ell})$ is as required (we identify $\mathbf{H}(i)$ with $[\mathbf{H}(i)]^{1}$, of course). Clauses $4.2 .8(\alpha)-(\gamma)$ are clear.

Suppose that $\left\langle t_{0}, t_{1}, \ldots\right\rangle \in \operatorname{PC}\left(K \overline{1.5 .9}, \Sigma(1.5 .9), m^{t_{n}}=n, \operatorname{dis}\left[t_{n}\right]=\left(n, E_{n}\right)\right.$, $\operatorname{nor}\left[t_{n}\right] \geq f_{N}^{1}(n)$. Then, for large enough $n,\left|E_{n}\right| \leq 2^{N}$, so let $M=\max \left\{\left|E_{n}\right|: n<\right.$ $\omega\}$. Assume that $\rho_{i} \in 2^{k+1}$ (for $i \leq M$ ) are pairwise distinct. By $\left(\oplus_{2}\right)$, for each $n \in\left[\ell_{k}, \ell_{k+1}\right)$ there is $i \leq M$ such that $g_{\rho_{i}}(n) \notin E_{n}$. Hence for some $i \leq M$

$$
\left|\left\{n \in\left[\ell_{k}, \ell_{k+1}\right): g_{\rho_{i}}(n) \notin E_{n}\right\}\right| \geq \frac{\ell_{k+1}-\ell_{k}}{M+1}=\frac{2^{2^{k}}}{M+1} .
$$

Hence we easily conclude that 4.2.8( $(\delta(\mathrm{i}))$ holds. The demands $4.2 .8(\delta(\mathrm{ii}))$ and 4.2.9(c) are even easier.

For $\mathcal{F}_{2}$ we proceed similarly, but we choose $g_{\rho}$ so that $g_{\rho}(n) \in[\mathbf{H}(n)]^{2^{n}}$.

\section{EPILOGUe}

A general problem that we have in mind in this paper is classifying "nice" ccc forcing notions, in particular finding dividing lines in this family, or at least natural properties. We should explain what we mean. A forcing notion is "nice" if it has a quite absolute definition, so Borel is natural, but Souslin is more central (see 1.3.1 and also [23]), but we may be happy with just "one of the form presented in this paper". A dividing line is a property of such definitions, so that both it and its negation is meaningful (that is we can prove theorems from both). Thus a dividing line may serve as a division to cases in solving problems. (On parallel in Model Theory see [27] and 25].)

The first (possible) dividing line we considered here is determined by "being $\omega$ nw-nep" (or just "being very Souslin ccc"). In some sense, one can consider very Souslin forcing notion as those which are really close to random and Cohen. We have examples of very Borel ccc forcing notions (see 1.5.8(2), 1.5.11, 1.5.15(3)), and forcing notions which are not $\omega-\mathrm{nw}-$ nep (see 1.5.4, 1.5.6, 1.5.8(1), 1.5.15 $(1,2)$ ). The argument for "not being $\omega$-nw-nep" was in all cases the same: adding a dominating real. So we arrive to the following question.

Problem 5.1. Suppose $\mathbb{P}$ is a Borel ccc forcing notion which is not equivalent to a $\omega$-nw-nep forcing. Does $\mathbb{P}$ add a dominating real?

If one looks at 1.3.4 (3) and 1.5.11, then the following (perhaps less central but still intriguing) question related to $\omega-\mathrm{nw}-$ nep forcing notions arises.

Problem 5.2. Assume $\mathbf{H}, K, \Sigma, \mathcal{F}$ and $h$ are as in 1.3.4(1c) or as in 1.3.4(2b). Is the forcing notion $\mathbb{Q}_{\mathcal{F}}^{*}(K, \Sigma)$ (or $\mathbb{Q}_{\mathcal{F}}^{\text {tree }}(K, \Sigma)$, respectively) very Borel ccc? (Of course, we are interested in non-finitary $(K, \Sigma)$.)

The second dividing line originates in 20 and studies of the Baire property (and measurability) of projective sets. To get a model in which all projective sets have the Baire property, [20] uses sweetness while 31 applies topological sweetness. However, the use of the two variants of sweetness might be slightly confusing. What we really need for this type of construction are two properties, say, (a)-sweetness and (b)-sweetness such that

(i) if $\mathbb{P}$ is (a)-sweet and $\dot{\mathbb{Q}}$ is a $\mathbb{P}$-name for a (b)-sweet forcing notion, then $\mathbb{P} * \dot{\mathbb{Q}}$ is (a)-sweet, 
(ii) if $\mathbb{P}_{0}, \mathbb{P}_{1}$ are (a)-sweet then for sufficiently many forcing notions $\mathbb{Q}$ and their two complete embeddings $f_{\ell}: \mathbb{Q} \longrightarrow \mathrm{RO}\left(\mathbb{P}_{\ell}\right)$, the amalgamation $\mathbb{P}_{0} \times_{f_{0}, f_{1}} \mathbb{P}_{1}$ is (a)-sweet,

(iii) the Universal Meager forcing notion is (b)-sweet,

(iv) (a)-sweetness implies the ccc.

(Note that in (ii) we do not require that all amalgamations are (a)-sweet, we just need to cover the amalgamations needed to ensure suitable homogeneity of the Boolean algebra we construct; see [6], also 3.3.3 here.) To some extend this approach was materialized in 4.1.9: the topological sweetness may serve as (a)sweetness and iterable sweetness is a good candidate for (b)-sweetness. It should be remarked here, that it is quite surprising that compositions of (topologically) sweet forcing notions with the Universal Meager (or the Hechler forcing notion) are topologically sweet because the second iterand is sweet. (The respective proofs in [20. [31] were somewhat less general.) Still, it is very reasonable to ask

Problem 5.3. Can 4.1.9 be improved by weakening the demands on $\dot{\mathbb{Q}}$ ? Can you find (a)-sweetness and (b)-sweetness satisfying (i)-(iv) and weaker then topological sweetness and iterable sweetness, respectively?

The sweet/sour division is sometimes very surprising - compare 4.3.3 (1) and 4.3.3 $(2)$. The forcing notions $\mathbb{Q}_{\mathcal{F}_{0}}^{*}\left(K \overline{1.5 .9}, \Sigma_{1.5 .9}\right)$ and $\mathbb{Q}_{\mathcal{F}_{2}}^{*}(K$ 1.5.9,, 2 1.5.9) (of 4.3.3) look very similar and one could expect that both are like the Cohen forcing. However, the first is topologically sweet (so not so far from Cohen) while the other is very explicitly sour (so one could even say that worse than random).

Topological sweetness occurs to be not so seldom (see 4.3.1), however it does not imply that we could make real use of these forcing notions in constructions like [19]. These forcings seem to be quite far from the iterable sweetness, so we conjecture that that the following has an affirmative answer.

Problem 5.4. Let $\mathbb{Q}$ be one of the forcing notion covered by 4.1.12 $(1,2)$ and 4.1.15. Is there $k<\omega$ such that the $\left(k\right.$-step) iteration $\mathbb{Q}^{(k)}$ is sour over Cohen? Over $\mathbb{Q}$ ?

Proposition 1.1.12(3) gives sweet forcing notions, so they could be of some use in constructions like that in 19]. However, do we really need to force additionally with these forcings? In other particular:

Problem 5.5. Is there a universality parameter $\mathfrak{p}$ satisfying the requirements of 4.1.12 (3) such that no finite iteration of the Universal Meager forcing notion adds a $\mathbb{Q}^{\text {tree }}(\mathfrak{p})$-generic real? Does the Universal Meager forcing add generic reals for the forcing of 2.4.10? Of 2.4.9?

An intriguing thing is that in the cases we proved sourness over the Cohen, we actually got that the considered forcing notion is very explicitly sour over Cohen (so in particular the amalgamation collapses $\mathfrak{c}$ ).

Problem 5.6. Let $\mathbb{Q}$ be a Souslin ccc (or just nep ccc) forcing notion, and $(\mathbb{P}, \dot{W})$ is as in 3.1 .1 .

1. Assume that $\mathbb{Q}$ is sour over $(\mathbb{P}, \dot{W})$. Is it explicitly sour over $(\mathbb{P}, \dot{W})$ ? Very explicitly?

2. Suppose that $\mathbb{Q}$ is not topologically sweet and adds a Cohen real. Is it (weakly) sour over Cohen? 
Since sweet/sour division is related to the Baire property of projective sets, let us finish with the following general problem.

Problem 5.7. 1. Let $(\mathbb{P}, \dot{W})$ be as in 3.1.1, $\mathbb{P}$ be of the type studied in this paper. For a cardinal $\kappa$, let $\mathcal{I}_{\mathbb{P}, \dot{W}}^{\kappa}$ be a $<\kappa$-complete ideal generated by $\mathcal{I}_{\mathbb{P}, \dot{W}}$. What is the consistency strength of the statement "every projective subset of $\mathcal{X}$ has the $\mathcal{I}_{\mathbb{P}, \dot{W}}^{\kappa}$-Baire property" ?

(We conjecture that it is always either ZFC or "ZFC + there exists an inaccessible cardinal, and we would like to characterize and/or describe this dividing line.)

2. Similarly for (typically non-ccc) ideals $\mathcal{I}_{\mathfrak{p}}$ determined by suitable universality parameters $\mathfrak{p}$ (see 3.2.1).

\section{REFERENCES}

[1] Tomek Bartoszyński and Haim Judah. Set Theory: On the Structure of the Real Line. A K Peters, Wellesley, Massachusetts, 1995.

[2] Jörg Brendle and Haim Judah. Perfect sets of random reals. Israel Journal of Mathematics, 83:153-176, 1993.

[3] Martin Goldstern and Haim Judah. Iteration of Souslin Forcing, Projective Measurability and the Borel Conjecture. Israel Journal of Mathematics, 78:335-362, 1992.

[4] P. Hall. On representatives of subsets. Journal of the London Mathematical Society, 10:26-30, 1935.

[5] Jaime Ihoda (Haim Judah) and Saharon Shelah. Souslin forcing. The Journal of Symbolic Logic, 53:1188-1207, 1988. [JdSh:292]

[6] Haim Judah and Andrzej Rosłanowski. On Shelah's Amalgamation. In Set Theory of the Reals, volume 6 of Israel Mathematical Conference Proceedings, pages 385-414. 1992.

[7] Haim Judah and Andrzej Rosłanowski. The ideals determined by Souslin forcing notions. unpublished notes, 1992; math.LO/9905144 1 ;

[8] Haim Judah, Andrzej Roslanowski, and Saharon Shelah.Examples for Souslin Forcing. Fundamenta Mathematicae, 144:23-42, 1994. [JRSh:373]; math.LO/9310224

[9] Haim Judah and Saharon Shelah. Baire Property and Axiom of Choice. Israel Journal of Mathematics, 84:435-450, 1993. [JdSh:446]; math.L0/9211213

[10] Alexander S. Kechris and Sławomir Solecki. Approximation of analytic by Borel sets and definable countable chain conditions. Israel Journal of Mathematics, 89:343-356, 1995.

[11] Kenneth Kunen. Random and Cohen Reals. In K. Kunen and J. E. Vaughan, editors, Handbook of Set-Theoretic Topology, pages 887-911. Elsevier Science Publishers B.V., 1984.

[12] Arnold W. Miller. Some properties of measure and category. Transactions of the American Mathematical Society, 266:93-114, 1981.

[13] John C. II Morgan. Baire category from an abstract viewpoint. Fundamenta Mathematicae, 94:13-23, 1977.

[14] John C. II Morgan. Point set theory, volume 131 of Monographs and Textbooks in Pure and Applied Mathematics. Marcel Dekker, Inc, New York, 1990.

[15] Andrzej Roslanowski and Saharon Shelah. Norms on possibilities I: forcing with trees and creatures. Memoirs of the American Mathematical Society, 141(671), 1999. [RoSh:470]; math.LO/9807172

[16] Andrzej Roslanowski and Saharon Shelah. Norms on possibilities II: more ccc ideals on $2^{\omega}$. Journal of Applied Analysis, 3:103-127, 1997. [RoSh:628]; math.L0/9703222

[17] Andrzej Roslanowski and Saharon Shelah. Norms on possibilities III: strange subsets of the real line. in preparation. [RoSh:670]

[18] Andrzej Roslanowski and Saharon Shelah. Measured creatures. Annals of Mathematics, submitted. [RoSh:736]; math.LO/0010070

[19] Andrzej Roslanowski and Saharon Shelah. 446 revisited. in preparation. [Sh:F380]

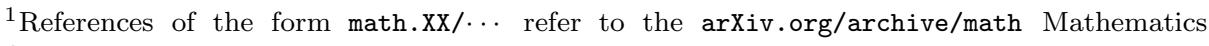
Archive
} 
[20] Saharon Shelah. Can you take Solovay's inaccessible away? Israel Journal of Mathematics, 48:1-47, 1984. [Sh:176]

[21] Saharon Shelah. How special are Cohen and random forcings i.e. Boolean algebras of the family of subsets of reals modulo meagre or null. Israel Journal of Mathematics, 88:159-174, 1994. [Sh:480]; math.LO/9303208

[22] Saharon Shelah. On what I do not understand (and have something to say). Fundamenta Mathematicae, 166:1-82, 2000. [Sh:666]; math.L0/9906113

[23] Saharon Shelah. Non-elementary proper forcing notions. Journal of Applied Analysis, accepted. [Sh:630]; math.LO/9712283

[24] Saharon Shelah. Non-elementary proper forcing notions II. In preparation. [Sh:669]

[25] Saharon Shelah. On what I do not understand (and have something to say), model theory. Mathematica Japonica, to appear. [Sh:702]; math.L0/9910158

[26] Saharon Shelah. Note on $\omega-$ nw-nep forcing notions. Preprint. [Sh:711]

[27] Saharon Shelah. Classification theory and the number of nonisomorphic models, volume 92 of Studies in Logic and the Foundations of Mathematics. North-Holland Publishing Co., Amsterdam, xxxiv+705 pp, 1990. [Sh:c]

[28] Saharon Shelah. Proper and improper forcing. Perspectives in Mathematical Logic. Springer, 1998. [Sh:f]

[29] Sławomir Solecki. Analytic ideals and their applications. Annals of Pure and Applied Logic, 99:51-72, 1999.

[30] Robert M. Solovay. A model of set theory in which every set of reals is Lebesgue measurable. Annals of Math., 92:1-56, 1970.

[31] Jacques Stern. Regularity properties of definable sets of reals. Annals of Pure and Applied Logic, 29:289-324, 1985.

Department of Mathematics, University of Nebraska at Omaha, Omaha, Ne 681820243, USA

E-mail address: roslanow@member.ams.org

URL: http://www. unomaha.edu/ aroslano

Institute of Mathematics, The Hebrew University of Jerusalem, 91904 Jerusalem, Israel, and Department of Mathematics, Rutgers University, New Brunswick, NJ 08854, USA

E-mail address: shelah@math.huji.ac.il

$U R L:$ http://www. math.rutgers.edu/ shelah 